\title{
Optimizing the efficacy of transcranial direct current stimulation on cortical neuroplasticity based on a neurovascular coupling model
}

\author{
DISSERTATION \\ for the award of the degree \\ "Doctor rerum naturalium" \\ Division of Mathematics and Natural Sciences \\ within the doctoral program \\ Systems Neuroscience \\ of the Georg-August University School of Science (GAUSS)
}

Submitted by

Asif Jamil

from Bel Air, Maryland, United States of America

Göttingen 2016 


\section{Thesis Committee}

Supervisor:

Prof. Dr. Michael A. Nitsche

Dept. Clinical Neurophysiology

University Medical Center Göttingen (UMG)

Prof. Dr. Melanie Wilke

Dept. Cognitive Neurology

University Medical Center Göttingen (UMG)

Prof. Dr. Dario Farina

Dept. Neurorehabilitation Engineering

University Medical Center Göttingen (UMG)

\section{Members of the Examination Board}

Prof. Dr. Dr. Hannelore Ehrenreich

Clinical Neuroscience

Max Planck Institute of Experimental Medicine (MPI-EM)

PD Dr. Peter Dechent

Dept. Cognitive Neurology

University Medical Center Göttingen (UMG)

Prof. Dr. Swen Hülsmann

Dept. Neuro- and Sensory Physiology

Center for Physiology and Pathophysiology, Georg-August-University

Date of oral examination: 24.01.2017 


\section{Statement of originality}

I hereby declare that the work entitled, "Optimizing the efficacy of transcranial direct current stimulation on cortical neuroplasticity based on a neurovascular coupling model" was independently written and the sources or aids used whether verbatim or in content are properly quoted in the text and references.

Göttingen, November 30, 2016

Asif Jamil 


\section{Acknowledgements}

I would like to give my heartfelt thanks to the many people who guided me over the past years. Foremost, I thank my advisor, Prof. Michael Nitsche, whose supervision was tremendous in shaping me towards become a critical and independent-thinking researcher. Notable was his constant support, honest feedback, and his willingness to push me outside of my soft limits so that I could gain valuable qualities as a scientist. I also thank my cosupervisors, Prof. Melanie Wilke and Prof. Dario Farina, in advising me with their kind support along the way.

I thank Prof. Walter Paulus, the head of our department, in providing me with the resources needed to conduct my studies, along with valuable feedback and advice along the way. In addition, I thank Prof. Martin Sommer and Prof. David Liebetanz for their friendly and positive support. I also thank the other clinical members of our department in assisting me with whatever support I needed, including Dr. Dirk Czesnik, Dr. Caspar Stephani, Dr. Elizabeth Stiksrud, and Dr. Florian Klinker. A special thanks goes to Manuel Hewitt, for his valuable friendship and technical assistance. Many thanks go to Marion Kurze, for her always positive and supporting nature, and in helping me with any administrative issues.

A special thanks goes to Peter Dechent, Carsten Schmidt-Samoa, Ilona Pfahlert, Britta Perl, and Severin Heumüller for their technical support and friendly attitude in allowing me to successfully conduct my imaging studies.

A sincere thanks goes to the colleagues in my group during my adventures as a PhD student: Giorgi Batsikadze, Shane Fresnoza, Linda Kuo, Yuichiro Shirota, Islam Halawa, Asad Khan, Aguida Foerster, Marcelo Lugon and Nathzidy Rivera Urbina. I also thank Natalia Haubrich, Gabriel Amador de Lara, Ivan Alekseichuk, Zsolt Turi, Annika Primassin, Alexander Whillier, Patrick Kunz, Alexander Opitz, Leonie Schmalfuss, and Catarina Saiote for maintaining a nice and friendly atmosphere.

Finally, a very deep heartfelt thanks goes to my family who provided me with constant encouragements. I could not have finished this work without their emotional love and support. I understand it was difficult for them also, therefore, I just say thanks for everything and may God bless you in return. 


\section{Table of Contents}

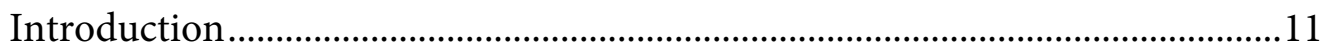

Original Articles and Manuscripts ..................................................................... 19

Efficacy of Anodal Transcranial Direct Current Stimulation is Related to Sensitivity to Transcranial Magnetic Stimulation..........................................20 Systematic evaluation of the impact of stimulation intensity on neuroplastic after-effects induced by transcranial direct current stimulation. .29 Current intensity- and polarity-dependent effects of transcranial direct current stimulation on cortical activation: an fMRI study . .45

Exploring functional effects of tDCS-induced neuroplasticity in bimanual

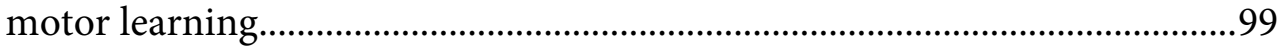

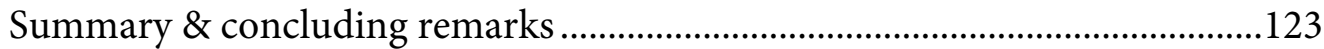

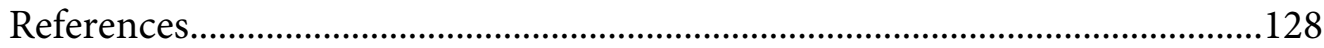

$\mathrm{CV}$ 
The chieffunction of the body is to carry the brain around.

-- Thomas A. Edison 


\section{Introduction}

The capacity of the brain to reorganize itself, structurally or functionally, in response to environmental or physiological stimuli, is one key and remarkable aspect of the nervous system. This ongoing process, termed neuroplasticity, encompasses the notion in which lasting changes occur at the molecular, morphological, synaptic, and/or functional level, either by neurogenesis or by the strengthening or weakening of existing synaptic connections (Ploughman, 2002; Pascual-Leone et al., 2005, 2011). It thereby relevantly accounts for the initiation of the new learning and memory forming processes in response to behavior, environmental stimuli, or injury or impairments, such that the brain attempts to repair itself. An overarching goal in the fields of neuroscience and clinical neurophysiology is to further understand, and also selectively alter the precise mechanisms of neuroplasticity which underpin these vital cognitive and restitutive functions in the brain. Such an ability would allow for the further understanding of key high-level executive functions in the cortex, such as working memory, attention, and planning, while also paving the way for development of more efficient adjuvant therapies in the treatment of various neurological and psychiatric disorders in which these naturally occurring dynamic alterations are negatively affected.

Investigations into neuroplasticity were traditionally only possible using invivo electrophysiological techniques in animal studies. Over the last decade, converging research and innovations in biomedical technology have led to the development of new tools based on electric and magnetic stimulation, such as paired associative stimulation (PAS), repetitive transcranial magnetic stimulation (rTMS), theta-burst stimulation (TBS), and transcranial direct current stimulation (tDCS), which offer the potential to induce neuroplastic changes in the human cortex (Pascual-Leone et al., 1994; Jennum et al., 1995; Stefan et al., 2000; Nitsche \& Paulus, 2000). In recent years, the latter technique has especially surged in cognitive research laboratories and clinical applications, owing to its non-invasive and painless method of delivering weak direct currents to alter the neuronal membrane potential (Nitsche \& Paulus, 2001). Moreover, after-effects of tDCS are akin to plasticity induced via long term potentiation and long term depression (LTP, LTD), as were observed in animal slice experiments (Bindman et al., 1964; Purpura \& McMurtry, 1965; Nitsche et al., 2008).

In the following work, we first present an overview on the mechanisms which underlie synaptic level neuroplasticity, with a focus on the human motor cortex as an experimental model. Modern non-invasive methods available to induce neuroplasticity in humans are next introduced. We then discuss how physiological markers of neuroplasticity can be measured and explored in humans with the use of transcranial magnetic stimulation (TMS) to assess excitability, and neuroimaging methods, such as electroencephalography (EEG) and functional magnetic resonance imaging (fMRI) to monitor physiological activity using high temporal and spatial resolutions. We then turn to the central goal of our thesis, which is to systematically evaluate the 
relationship between the neuroplastic alterations observed in motor cortical excitability and in motor cortical cerebro-vascular response induced by tDCS-a relationship we will refer to as neurovascular coupling. As we will discuss, accomplishing a more enriched understanding of this relationship can provide a basis for further optimized tDCS protocols to be used in routine clinical and research settings, which is crucial, considering the high sensitivity of tDCS after-effects to variable methodological parameters such as stimulation duration and DC amperage. Finally, we outline the research methods which were undertaken to accomplish this goal, and which are then presented in detail over the subsequent sections. The last chapter summarizes the findings of the presented studies, and offers an outlook and future research directions in the field.

\section{Neuroplasticity in the cerebral cortex}

Prior to the turn of the $20^{\text {th }}$ century, the common notion and wisdom was that after childhood, the only change to the brain which took place was a constant and progressive decline, and no alterations could take place following any type of injury or damage. It is now understood that plasticity is a normal ongoing process throughout the life-span, which underlies mechanisms of learning and memory (Pascual-Leone et al., 2005).

In 1949, Hebb published his seminal paper on the synaptic plasticity model, which proposed that when an axon of a particular neuron $A$ is near enough to excite an adjacent neuron $B$ repeatedly, there is some growth process which increases the overall efficiency of neuron A to fire neuron B (Hebb, 1949). Since his discovery, the synaptic plasticity model has evolved to include various mechanisms related to the regulation of synaptic strength, such as long-term potentiation (LTP) and long-term depression (LTD), while also respecting modifications in the size or number of synapses, as well as functional alterations in the process of synaptic transmission. Due to the relevance for learning as well as the formation and storage of memory, LTP and LTD has since attracted increasing research attention, where animal hippocampal slices have served as a traditional model (Andersen \& Lomo, 1966; Bliss \& Lomo, 1973; Cooke \& Bliss, 2006a). Here, findings have pointed to the role of the calcium-ion based glutamatergic N-methyl-D-aspartate (NMDA) receptor in allowing the influx of calcium into the cell, which then triggers the regulation of AMPA receptors (Coan \& Collingridge, 1987; Miyamoto, 2006). Importantly, it was observed that the direction of plasticity (LTP or LTD) may depend critically on the concentration of the post-synaptic intracellular calcium (Lisman, 2001). Following low-frequency stimulation, calcium triggers the removal of AMPA receptors via endocytosis, weakening and desensitizing the synapse to further glutamate and resulting in LTD, while with sufficiently high stimulation, calcium triggers a separate cascade whereby additional AMPA receptors are added, resulting in LTP (Cummings et al., 1996; Malenka \& Bear, 2004). Furthermore, there appears to exist a transition zone whereby neither LTP nor LTD are induced (Cho et al., 2001; Lisman, 
2001), and moreover, with an excessively high calcium influx, LTP is also abolished due to hyperpolarizing potassium channels (Misonou et al., 2004). LTP and LTD have also been associated with gamma-aminobutyric acid (GABA) synaptic transmission (Stelzer et al., 1987, 1994; Caillard et al., 1999), where bi-directionality in LTP or LTD also depends on intracellular calcium (McLean et al 1996).

These primary findings, coupled with the development of newer electrophysiological techniques, have subsequently led to the successful induction of LTP and LTD plasticity in other neocortical areas like the auditory (Pereda et al., 1998; Friauf et al., 2015), visual (Kirkwood \& Bear, 1994; Heynen \& Bear, 2001), and motor cortices (Hess \& Donoghue, 1994; Sanes \& Donoghue, 2000; Rioult-Pedotti et al., 2000).

\section{Neuroplasticity in the human motor cortex}

Success in animal slice studies continue to contribute affluent information on physiological mechanisms, which has resulted in the possibility to also investigate neuroplasticity in humans. Transferability of the findings could be partially achieved when it was shown that in-vitro slices from the temporal and hippocampal tissues of epileptic patients also exhibited LTP induced by micro-stimulation (Chen et al., 1996; Beck et al., 2000; Cooke \& Bliss, 2006b). These studies, however, require careful interpretation, as ongoing spontaneous activity in an intact brain, and within a larger synaptic population may relevantly affect the extent of neuroplasticity (Winnubst et al., 2015). Many studies have demonstrated the ability of the human brain to achieve LTP-like plasticity. For example, use-dependent plasticity, which can be evident after some minutes of continuous thumb movements (Classen et al., 1998), or the reorganization and resizing of motor cortical representation maps during, and shortly following piano learning (Pascual-Leone et al., 1995). Most of these studies have focused on the motor cortex as an experimental model system, due to the ease in targeting the relatively superficial anatomical region, as well as the system's ability to output distinctly measurable physiological responses following induced stimulation interventions with the use of electromyography or neuroimaging techniques. Besides these methodological advantages, the induction of neuroplasticity in the primary motor cortex (M1) can uncover novel insights into debilitating motor processes following brain injury and disease, or even shed light on developing new learning and training mechanisms, such as assisting elderly in the acquisition and performance of complex motor skills (Zimerman \& Hummel, 2010). The M1 is layered in its cortical architecture, and consists of horizontal inter-neurons that are thought to be relevant for the assembly and maintenance of long lasting synaptic modifications (Kleim et al., 1998; Rioult-Pedotti et al., 1998; Sanes \& Donoghue, 2000). An arterial blood supply originating from the central sulcus arteries additionally allows for exploring the interaction of metabolic pathways with experimental neuromodulatory interventions (Lang et al., 2005; Ugur et al., 2005). Finally, the M1 has been shown to have the key characteristics of a "small world" network, in which anatomical and functional connectivity 
within this cortex contain the means for a dynamic reorganization in order to efficiently respond to both specialized and integrated inputs (Bassett \& Bullmore, 2006; Sporns \& Honey, 2006; Chen \& Rothwell, 2012). Recent neuroimaging research has shown that the M1 undergoes a functional reorganization as a result of induced neuroplasticity (Chen \& Rothwell, 2012), which is important because it reflects the ability of the M1 to be relatively flexible for modification and map reorganization.

\section{Modulation of neuroplasticity by non-invasive brain stimulation}

Largely inspired by the electrophysiological findings in animal models, the development of methods to induce neuroplasticity in humans in recent decades has focused on producing techniques which are non-invasive, yet still able to robustly induce physiological changes in the cortex. The first of these techniques was the development of transcranial electric stimulation, which leveraged high voltage currents to penetrate through the skull, and target neuronal populations within the cortex (Merton \& Morton, 1980). However, this technique was associated with uncomfortable and even painful perceptions by the subject, due to the high intensity of the current, and its path in to the skull through dermal pain receptors. A less daunting alternative became viable with the development of transcranial magnetic stimulation (TMS) (Barker et al. 1985). TMS makes use of a high strength magnetic pulse can penetrate through the skull, thereby inducing a secondary moving electric field within the brain. This pulse is associated with a supra-threshold neuronal stimulus, which does not activate pain receptors or head muscles. When TMS is applied to the motor cortex, supra-threshold activation of neuronal populations within various motor cortical representation areas of the body can elicit an involuntary muscular contraction (motor evoked potential - MEP), which can be recorded electromyographically. Thus, one important measure obtained from single-pulse TMS is the corticospinal excitability (Rothwell, 1993), which can be monitored for changes following plasticity induction protocols. TMS pulses can be further altered in their repetitive frequency (rTMS), such that low or high frequency periods of stimulation can induce neuroplastic changes in excitability (Fitzgerald et al., 2006). Combining TMS with a precisely timed low frequency peripheral stimulation (PAS) has also been shown to induce bidirectional neuroplastic changes in motor cortical excitability (Stefan et al., 2000), possibly due to some similarity with spiketiming dependent mechanisms of plasticity, but mainly through its dependency on NMDA receptors and calcium channel activity (Stefan et al., 2002; Wolters et al., 2005).

Besides these techniques, another approach has been to apply weak (subthreshold) direct currents to the scalp, which are able to penetrate the skull and influence the resting membrane potential of neuronal populations (Nitsche \& Paulus, 2000). This technique, termed transcranial direct current stimulation (tDCS), delivers weak currents through two or more sponge electrodes placed on the scalp, and this "montage" creates a semi-current loop through the brain. The polarity of the stimulation (anodal or cathodal) is 
classically termed by the respective type of electrode placed over the target cortical area on the scalp. As such, with a tDCS intensity of $1.0 \mathrm{~mA}$ and duration of $13 \mathrm{~min}$, motor cortical excitability was enhanced in a group of young subjects for more than $60 \mathrm{~min}$ following the end of stimulation, whereas with 9 min of cathodal tDCS, excitability diminished (Nitsche and Paulus, 2001, Nitsche et al. 2003b). The underlying mechanisms of tDCS are based on animal studies from the 1960s, where it was shown that application of polarity-specific currents could bi-directionally alter the frequency of spontaneous neuronal spiking, both during the period of stimulation as well as for a short time afterwards (Bindman et al., 1964; Purpura \& McMurtry, 1965). These after-effects were later proposed to be linked to changes in intracellular calcium concentration, and NMDA-receptor mediated gene expressions (Islam et al., 1995b, 1995a), thus, similar in mechanistic properties to LTP- and LTD-like plasticity. Moreover, in human studies, tDCS combined with the pharmacological administration of NMDA receptor antagonists and calcium channel blockers was shown to abolish tDCS-induced plasticity (Liebetanz et al., 2002; Nitsche et al., 2003). Administration of Lorazepam, a GABA receptor agonist, initially reduced the excitability enhancement following anodal tDCS, whereas it had no effect on cathodal tDCS (Nitsche et al., 2004). Interestingly, a role of a calcium concentration dependency for LTP or LTD induction has also been proposed, as evidenced by studies which have increased the tDCS intensity and stimulation duration parameters. When cathodal tDCS at $1.0 \mathrm{~mA}$ was increased to $2.0 \mathrm{~mA}$, motor cortical excitability reversed from diminution to facilitation (Batsikadze et al., 2013), and when the duration of anodal tDCS was increased from 13 to $26 \mathrm{~min}$, after-effects were abolished (Monte-Silva et al., 2013). The latter conversion effects, which were interpreted to be caused by calcium overflow, were abolished by the calcium channel blocker flunarizine.

Evidences of neuroplastic tDCS-induced alterations in brain physiology are not limited to only motor cortical excitability studies. Polarity-specific effects of motor-cortical tDCS on local GABA and glutamate concentrations have been documented using magnetic resonance spectroscopy (MRS) (Stagg et al., 2009, 2011). Anodal tDCS for 10 min over the left M1 reduced GABA, while cathodal tDCS reduced glutamate (GABA was reduced proportionately as well, although these processes may be linked (Stagg \& Nitsche, 2011)). Using positron emission tomography (PET), it was shown that both anodal and cathodal tDCS induce widespread increases and decreases in regional cerebral blood flow (rCBF) (Lang et al., 2005). Alterations in the local hemodynamic response following tDCS have also been observed using Arterial Spin Labeling (ASL-fMRI) where it was shown that $\mathrm{rCBF}$ increased after short repeated durations of anodal tDCS and decreased after cathodal tDCS (Zheng et al., 2011). This finding does not appear to be constrained only to the motor cortex, as anodal and cathodal tDCS applied to the left dorsolateral prefrontal cortex (DLPFC) also resulted in polarity-specific alterations in perfusion (Stagg et al., 2013). An open question is whether such changes are due to synaptic plasticity mechanisms affecting neuro-vascular-glial units, or due to the effect of electric fields on the vascular tone (Lee et al. 1975, Toda, 1981, 
Asberg et al 1999). Modulations of resting state oscillatory activity, recorded using EEG, have also been reported after anodal and cathodal tDCS and reiterate the concept of increased or decreased spontaneous firing activity in pyramidal neuron populations, respectively (Notturno et al., 2014; Roy et al., 2014). Recent advancements in brain network connectivity analyses applied to neuroimaging data, such as the use of graph theoretical parameters to assess functional and structural connectivity, have shed important light on mechanisms by which tDCS can induce functional cortico-cortical, and cortico-thalamic alterations (Polanía et al., 2011c, 2011a, 2011b). Considering that an important aspect of tDCS is to modulate learning-related mechanisms, these findings offer special relevance to the prospect of integrating the communication of segregated cortical areas at the system level.

In the past years, tDCS has attracted increasing use in both research and clinical settings due to its non-invasive and painless nature, and its simplistic ease of use for investigating cognitive functions or aiding in therapeutic treatments (Kuo \& Nitsche, 2012; Kuo et al., 2014; Shin et al., 2015; Woods et al., 2016). Although most of these studies have seen promising success, the physiological after-effects following single sessions of tDCS are relatively limited in their capacity for maintaining long lasting changes in comparison to modern pharmacological treatments. Approaches to enhance these effects have included altering the focality of tDCS (Nitsche et al. 2007), increasing the stimulation intensity and prolonging stimulation duration to $30 \mathrm{~min}$ (Boggio et al., 2009; Shekhawat et al., 2013; Brunoni et al., 2013), or using shortinterval repetitions of tDCS sessions (Monte-Silva et al., 2013). However, a clear dose-response relationship requires a systematic titration of these parameters, which has so far not been reported, particularly in a repeated measure design, such that inter-individual variability can be closely monitored. Inter-individual variability in the after-effects of tDCS, as well as other non-invasive brain stimulation protocols, has been mentioned and discussed in a few reports (López-Alonso et al., 2014; Wiethoff et al., 2014; Chew et al., 2015; Strube et al., 2015), and may depend on factors such as demographics, genetics, brain anatomy, attention, and/or baseline neuronal states (Kuo et al., 2006; Ridding \& Ziemann, 2010; Li et al., 2015; Opitz et al., 2015). Understanding the interaction of these factors, and devising steps to overcome this variability in experimental settings remains another important goal of the field.

\section{Aim of the thesis}

As presented in the following dissertation, we aimed to explore the relationship between systematically varying stimulation intensities of tDCS with the respective physiological and functional effects in the human motor cortex, with the goal of obtaining stimulation parameters which might yield greater and longer lasting neuroplasticity. We devised a multi-modal experimental approach and set out to answer three questions. 
First, one approach to understanding a current intensity dose-reponse relationship and optimizing tDCS would be to evaluate whether interindividual factors, especially those which might be relevant in affecting cortical plasticity, have already shown to account for greater and longer-lasting aftereffects of tDCS, as such a finding may also provide important clues towards addressing factors associated with inter-individual variability of tDCS. For example, the prescription of many pharmaceutical medications are individually adjusted depending on the patient's body weight, in order to ensure the most optimal pharmakokinetic response (Sheiner et al., 1972). This principle also exists in other plasticity-induction techniques, such as PAS, where the TMS stimulator intensity is adjusted at baseline depending on the default excitability of the specific subject, which then results in measurements that are more sensitive to the experimental manipulation. In this regard, understanding if such a relationship is applicable to tDCS may also be useful. We began by examing the relationship between individual differences in TMS baseline sensitivity and the efficacy of tDCS, based on an archival review of previously published tDCS data collected in our lab. We expected to see a negative relationship such that higher TMS sensitivity (i.e., a low TMS intensity for the motor threshold) would predict better efficacy of anodal tDCS. As a control, we also investigated the relationship between TMS sensitivity and PAS efficacy, where we did not expect any such relationship since the intensity for PAS is routinely adjusted for the subject.

Our second question was to systematically assess the effect of current intensity on neuroplastic after-effects of tDCS, by measuring the respective responses in both cortical excitability and cerebral blood flow. Whereas the impact of specific tDCS protocol parameters on the efficacy of stimulation have been comparatively investigated in different studies, a systematic finding over a group of healthy young subjects (also undergoing repeated measures) remains to be reported. Moreover, unlike cortical excitability studies, there have been no reported studies investigating the effect of tDCS on the time span covering the after effects in CBF. Our experimental approach was thus divided into two experiments-Experiment 1 involved TMS measurements, where subjects took park in five sessions of anodal or cathodal tDCS (sham, 0.5, 1.0, 1.5 and $2.0 \mathrm{~mA}$ ) and motor cortical excitability was assessed for up to $2 \mathrm{~h}$. In the second experiment, the same subjects received the same five sessions of tDCS, this time inside an MR scanner, where Arterial Spin Labeling (ASL) MRI was used to quantify changes in cerebral perfusion during and up to $2 \mathrm{~h}$ after tDCS. The ASL technique reliably obtains quantitative measures of $\mathrm{CBF}$, in measurement units that are comparable to PET (Detre \& Alsop, 1999). Based on previous studies, as well as insights from previous findings in the literature, we expected that the largest intensity of anodal tDCS $(2.0 \mathrm{~mA})$ and small or medium intensity of cathodal tDCS (0.5-1.0 mA) to mimic the prototypical LTP and LTD mechanisms, and thereby respectively modulate cortical excitability and cerebral blood flow in a polarity and intensity-dependent manner. As no clear findings on the effect of tDCS on vascular connectivity have been reported, we further explored alterations in cortico-cortical 
functional connectivity to obtain first knowledge regarding the regional and network level extent of tDCS effects on arterial perfusion.

Finally, our third question was to validate whether our optimal tDCS protocol also induces functional alterations in motor learning, and whether these alterations are also able to restitute skill learning in the aging population. With regard to motor learning, several studies have observed deficits in sequence learning in elder adults, particularly when task or complexity is increased. Among the recently developed motor control paradigms is the "bimanual tracking task", a complex task that requires intensive practice to successfully integrate the two separate limb movements into one common spatiotemporal pattern (Swinnen et al., 1997; Swinnen, 2002). We devised a two-part approach to address this task. In a first control study, we used EEG to investigate the relationship between motor performance and functional connectivity based on a data-driven statistical approach in both young and elderly samples. In the second phase, tDCS was applied in a second group of young and elderly subjects (using a crossover design to compare with sham tDCS). We expected our tDCS protocol to successfully enhance the acquisition and performance in the bimanual tracking task.

In summary, the main theme of this work is to elucidate the mechanistic relationship of current intensity on neuroplastic after-effects, and assess the extent of the relationship between cortical excitability and haemodynamic responses. The knowledge we gain here might help to aid in the transferability of motor cortical excitability effects to other regions in the brain, where use of TMS to monitor neuroplastic alterations is not possible. 


\section{Original Articles and Manuscripts}

The following section consists of two published articles and two manuscripts in submission.

1. Labruna L, Jamil A, Fresnoza S, Batsikadze G, Kuo MF, Vanderschelden B, Ivry RB \& Nitsche MA (2016). Efficacy of Anodal Transcranial Direct Current Stimulation is Related to Sensitivity to Transcranial Magnetic Stimulation. Brain Stimul 9, 8-15.

DOI: 10.1016/j.brs.2015.08.014

2. Jamil, A., Batsikadze, G., Kuo, H.-I., Labruna, L., Hasan, A., Paulus, W., Nitsche, M.A (2017). Systematic evaluation of the impact of stimulation intensity on neuroplastic after-effects induced by transcranial direct current stimulation. J. Physiol. 595, 1273-1288.

DOI: $10.1113 / J P 272738$

3. Jamil A, Batsikadze G, Kuo H-I, Dechent P, Paulus W \& Nitsche MA. Current intensity- and polarity-dependent effects of transcranial direct current stimulation on cortical activation: an fMRI study. In submission.

4. Jamil A, Rand M, Cuypers K, Nitsche MA \& Meesen R. Investigating bimanual motor coordination in healthy young and older adults using EEG and transcranial direct current stimulation. In submission. 


\title{
Efficacy of Anodal Transcranial Direct Current Stimulation is Related to Sensitivity to Transcranial Magnetic Stimulation
}

\section{Efficacy of Anodal Transcranial Direct Current Stimulation is Related to Sensitivity to Transcranial Magnetic Stimulation}

Ludovica Labruna a,b,*,1, Asif Jamil c,1, Shane Fresnoza ${ }^{c}$, Giorgi Batsikadze ${ }^{c}$, Min-Fang Kuo ${ }^{c}$, Benjamin Vanderschelden a , Richard B. Ivry a,b, Michael A. Nitsche ${ }^{c, d, e}$

a Department of Psychology, University of California, Berkeley, California, USA

${ }^{\mathrm{b}}$ Helen Wills Neuroscience Institute, University of California, Berkeley, Califormia, USA

' Department of Clinical Neurophysiology, University Medical Center, Georg-August-University, Goettingen, Germany

d Leibniz Research Center for Working Environment and Human Factors, Dortmund, Germany

E Department of Neurology, BG University Hospital Bergmannsheil, Ruhr-University Bochum, Bochum, Germany

A R T I C L E I N F O

Article history:

Received 9 November 2014

Received in revised form 21 July 2015

Accepted 30 August 2015

Available online

\section{Keywords:}

tDCS

TMS

Intensity

Variability

\begin{abstract}
A B S T R A C T
Background: Transcranial direct current stimulation (tDCS) has become an important non-invasive brain stimulation tool for basic human brain physiology and cognitive neuroscience, with potential applications in cognitive and motor rehabilitation. To date, tDCS studies have employed a fixed stimulation level, without considering the impact of individual anatomy and physiology on the efficacy of the stimulation. This approach contrasts with the standard procedure for transcranial magnetic stimulation (TMS) where stimulation levels are usually tailored on an individual basis.

Objective/Hypothesis: The present study tests whether the efficacy of tDCS-induced changes in corticospinal excitability varies as a function of individual differences in sensitivity to TMS.

Methods: We performed an archival review to examine the relationship between the TMS intensity required to induce $1 \mathrm{mV}$ motor-evoked potentials (MEPs) and the efficacy of (fixed-intensity) tDCS over the primary motor cortex (M1). For the latter, we examined tDCS-induced changes in corticospinal excitability, operationalized by comparing MEPs before and after anodal or cathodal tDCS. For comparison, we performed a similar analysis on data sets in which MEPs had been obtained before and after paired associative stimulation (PAS), a non-invasive brain stimulation technique in which the stimulation intensity is adjusted on an individual basis.

Results: MEPs were enhanced following anodal tDCS. This effect was larger in participants more sensitive to TMS as compared to those less sensitive to TMS, with sensitivity defined as the TMS intensity required to produce MEPs amplitudes of the size of $1 \mathrm{mV}$. While MEPs were attenuated following cathodal tDCS, the magnitude of this attenuation was not related to TMS sensitivity nor was there a relationship between TMS sensitivity and responsiveness to PAS

Conclusion: Accounting for variation in individual sensitivity to non-invasive brain stimulation may enhance the utility of tDCS as a tool for understanding brain-behavior interactions and as a method for clinical interventions.
\end{abstract}

Published by Elsevier Inc.

Abbreviations: ADM, abductor digiti minimi muscle; M1, primary motor cortex MEPs, motor evoked potentials; $M E P_{1 \mathrm{mV}}$ intensity, $1 \mathrm{mV}$ peak-to-peak amplitude: MSO, maximum stimulator output; MT, motor threshold; PAS, paired associative stimulation; tDCS, transcranial direct current stimulation; TMS, transcranial magnetic stimulation.

Corresponding author. Tel.: +510 642 0135; fax: +510 6420135

E-mail address: lulabrun@gmail.com (L. Labruna).

Joint first authors of this paper.

\section{Introduction}

Non-invasive brain stimulation has become an important tool for basic research in human brain physiology, cognitive neuroscience and translational methods designed to provide new clinical interventions. A variety of methods have been developed for human application over the past thirty years, including transcranial magnetic stimulation (TMS), paired associative stimulation (PAS) [1] and transcranial direct current stimulation (tDCS) [2]. These methods have been used to perturb or enhance motor and cognitive function [2], probe the dynamics of cortical physiology [3], and treat 
symptoms associated with a range of neurological and psychiatric disorders [4-6].

In tDCS, a direct electrical current is used to modify neural excitability, inducing subthreshold membrane polarization shifts, whose direction depend on stimulation polarity. At rest, corticospinal excitability is assumed to increase when the anodal electrode is positioned over the primary motor cortex (M1) and decrease when the cathodal electrode is positioned over M1. Based on the membrane polarization effects, applying tDCS for a few minutes results in alteration of the strength of glutamatergic synapses, and thus longlasting neuroplastic effects [7]. Anodal tDCS produces an increase in TMS-elicited MEPs amplitudes, whereas cathodal tDCS produces a decrease in MEPs amplitudes.

PAS offers an alternative method of plasticity induction. In this method, an electrical stimulus is applied over a peripheral nerve in combination with TMS over the contralateral motor cortex. MEP alteration depend on the interstimulus interval (ISI) between the TMS pulse and the nerve stimulation [1,8]: MEPs decrease with a short ISI (e.g., $10 \mathrm{~ms}$ ) due to the asynchronous activation of motor cortex neurons by the peripheral and cortical stimulus, and increase with a longer ISI (e.g., $25 \mathrm{~ms}$ ), presumably due to synchronous activation.

As currently practiced, the intensity of stimulation in most TMS and PAS studies is established on an individual basis. That is, the desired stimulation level is established on a functional/physiological criterion rather than set to a constant level across participants. To this end, a procedure is conducted prior to the experiment proper to establish the required stimulation intensity to meet some defined criterion. The criterion could be resting motor threshold operationalized as the intensity required to elicit MEPs of $50 \mu \mathrm{V}$ in at least $50 \%$ of the trials [9], or a targeted size of the MEPs (e.g., $1 \mathrm{mV}$ [10]). This approach is designed to minimize the impact of taskirrelevant factors that introduce inter-participant variability. For example, the physiological impact of a TMS pulse of a fixed intensity may be influenced by anatomical factors such as skull thickness and the cortical orientation of the targeted neural region [11,12] As such, a TMS pulse of a fixed intensity will result in variable MEPs amplitudes across individuals. By tailoring the TMS intensity on an individual basis, a common baseline is established and, as a consequence, the experiment is more sensitive to the effect of an experimental manipulation.

While stimulation factors such as intensity, duration, and electrode configuration have been shown to determine efficacy of tDCS at the group level (e.g. Ref. 10), the stimulation intensity used in tDCS studies is set to a fixed level for all participants. In some studies, the intensity might be $1 \mathrm{~mA}$, in others $2 \mathrm{~mA}$. But unlike TMS or PAS, the intensity is fixed for all participants. The use of fixed stimulation intensity in tDCS add a source of variability that is extraneous to the experimental manipulation, and might be a factor contributing to the inter-individual variability of tDCS effects [13-16]

As a first step in exploring this issue, we examined the relationship between individual differences in sensitivity to TMS and the efficacy of tDCS. We performed an archival review, analyzing data from prior studies published by our group to explore if tDCSinduced changes in corticospinal excitability are related to individual differences in sensitivity to TMS. For all participants, the data sets included the TMS intensity required to evoke MEPs amplitudes of $1 \mathrm{mV}$ elicited by single pulse TMS, operationalized as percentage of maximum stimulator output (MSO). We predicted that participants most sensitive to TMS (low MSO) will show the greates response to tDCS and that participants who are less sensitive to TMS (high MSO) will show a smaller response to tDCS. In other words, we predict a negative relationship between MSO and tDCS effects on corticospinal excitability. As a control measure, we performe a similar analysis relating TMS sensitivity to MEP changes obtained in two PAS protocols. Given that stimulation parameters in the PAS protocol are determined individually, we did not expect to observe a relationship between MSO and PAS effects on corticospinal excitability.

\section{Materials and methods}

The analyses reported here were performed on data sets from three studies [17-19]. The focus of these studies was on the impact of pharmacological interventions on plasticity associated with tDCS and PAS. In the current study, we restricted the analysis to the control data from these studies, the conditions in which the participants were administered a placebo substance.

\section{Participants}

For the tDCS conditioning groups, data were available from 34 participants who had received anodal and cathodal tDCS, and from two additional participants who had only received anodal tDCS ( $n=36,16$ women, 20 men, $27 \pm 5$ years old). For the PAS conditioning groups, data were available from 36 participants $(n=36: 15$ women and 21 men; $27 \pm 4$ years old). As assessed by the Edinburgh Handedness Inventory [20], all participants were right-handed.

All participants were naive to the purpose of the study and were financially compensated. The protocol was approved by the ethics commission of the University Medical Center of the University of Gottingen and conformed to international standards for testing with human participants (Declaration of Helsinki). All participants provided written informed consent prior to the start of the experiment.

\section{Transcranial magnetic stimulation}

TMS was delivered through a $70 \mathrm{~mm}$, figure-of-eight coil driven by a Magstim 200 magnetic stimulator (Magstim, Whitland, Dyfed, UK). The coil was positioned over left motor cortex to elicit MEPs in the right abductor digiti minimi muscle (ADM). The coil was placed tangentially on the scalp with the handle oriented toward the back of the head and laterally at a $45^{\circ}$ angle from the midline, an orientation that is approximately perpendicular to the central sulcus. Single-pulse TMS was applied at $0.25 \mathrm{~Hz}$ to identify the optimal spot for eliciting MEPs in the ADM. This hotspot was marked on the participant's scalp to provide a reference point for the experimental session.

The intensity of TMS (defined in terms of percentage of maximum stimulator output, MSO) was adjusted to elicit, on average, baseline MEPs of $1 \mathrm{mV}$ peak-to-peak amplitude (MEP ${ }_{1 \mathrm{mv}}$ intensity). The EMG display was set to allow the experimenter to easily visualize a $1 \mathrm{mV}$ change in the EMG signal. The experimenter then adjusted the output manually, seeking a stimulation level that produced MEPs of approximately $1 \mathrm{mV}$ amplitude. The final value corresponded to the stimulation level in which $1 \mathrm{mV}$ MEPs were assumed to be elicited in the target muscle. This was probed via baseline MEPs recording, for which 25 MEPs were obtained. If mean baseline MEPs size was within the range of $1 \mathrm{mV} \pm 20 \%$ MSO, this value was accepted. If it exceeded these limits, TMS intensity was determined again. The final stimulation level was fixed at this level for the remainder of the experiment.

EMG was recorded from surface electrodes placed over the right $\mathrm{ADM}$. The EMG signal was monitored on-line to ensure that participants maintained a relaxed posture over the course of the experiment. The EMG signals were amplified (gain, 1000) and bandpass-filtered $(2 \mathrm{~Hz}-2 \mathrm{kHz})$. The signals were digitized at $5 \mathrm{kHz}$ for off-line analysis by Signal software and CED 1401 hardware (Cambridge Electronic Design). EMG data were collected for $200 \mathrm{~ms}$ on each trial, starting $80 \mathrm{~ms}$ before the TMS pulse. 


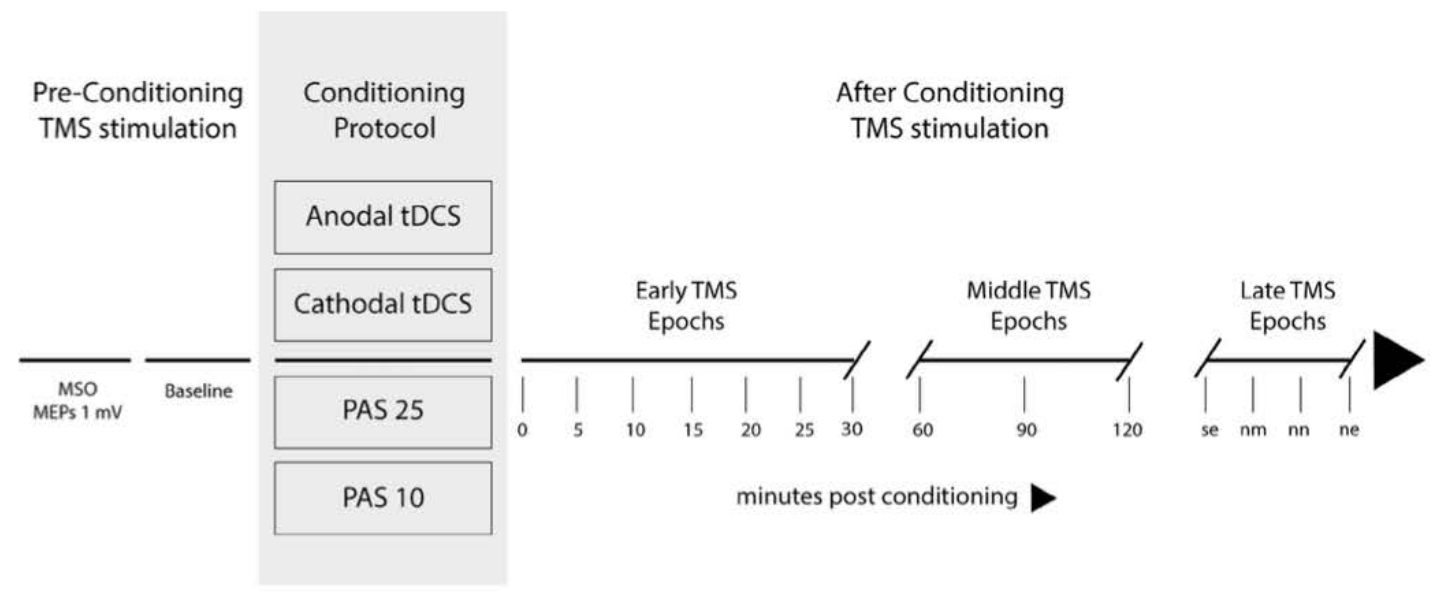

Figure 1. Experimental procedure. Data were available from studies using four different conditioning protocols: anodal tDCS, cathodal tDCS, PAS 25 stimulation, or PAS 10 stimulation. For each participant, the maximum stimulator output (MSO) was set to elicit baseline MEPs that averaged $1 \mathrm{mV}$ peak-to-peak amplitude. A baseline measure of corticospinal excitability was obtained prior to conditioning protocol and then at multiple time points following conditioning. Three time windows were defined: The Early window included all epochs between 0 and $30 \mathrm{~min}$ and the Middle window included epochs between 60 and $120 \mathrm{~min}$. A Late window was composed of epochs obtained after the initial 2-hour session: same evening (se), next morning (nm), next noon (nn), next evening (ne).

Conditioning protocols

Transcranial direct current stimulation (tDCS)

tDCS was delivered through a battery-driven constant current stimulator (Neuroconn, Germany). The current was applied through saline-soaked surface sponge electrodes $\left(7 \times 5 \mathrm{~cm}\right.$; area $\left.35 \mathrm{~cm}^{2}\right)$. The active electrode was centered over the ADM hotspot of the left M1. The reference electrode was positioned above the contralateral supraorbital ridge. tDCS was applied with a current intensity of $1 \mathrm{~mA}$ for 13 minutes in the anodal tDCS condition and $9 \mathrm{~min}$ in the cathodal tDCS condition, with a 10 -s ramp at the beginning and end of the stimulation. These stimulation protocols have been shown to induce changes in corticospinal excitability for up to 1 hour after the end of stimulation $[21,22]$

Paired associative stimulation (PAS)

For the PAS protocol, an electrical pulse was delivered (Digitimer D185 multipulse stimulator) to the right ulnar nerve at the wrist, paired with a TMS pulse to the left M1 ADM hotspot. The intensity of the electrical pulse was set to three times the sensory perceptual threshold. Sensory perceptual threshold was defined as the minimal electrical stimulation intensity (in Volts), which resulted in a somatosensory perception. Somatosensory threshold was identified by stepwise increase of the stimulation intensity. TMS intensity was adjusted to result in a mean MEPs amplitude of $1 \mathrm{mV}[1,23]$. The electrical and magnetic pulses were separated by an interval of either 10 or $25 \mathrm{~ms}$, with the peripheral nerve pulse always followed by the TMS stimulus. These paired pulses were administered once every $20 \mathrm{~s}$ for $30 \mathrm{~min}$. The ISI determines the direction of induced plasticity. With the $10 \mathrm{~ms}$ ISI (PAS10) excitability is attenuated whereas with the $25 \mathrm{~ms}$ ISI (PAS25) excitability is enhanced [1,23,24]. Note that, unlike tDCS, the parameter settings for PAS are identified on an individual basis.

\section{Procedure}

Participants sat in a comfortable chair with both hands resting on a pillow, palms down, with the arms in a semi-flexed position. The experimental protocol is summarized in Fig. 1. After establishing the TMS intensity required to produce MEPs of $1 \mathrm{mV}$, an initial set of 25 baseline MEPs was obtained $(0.25 \mathrm{~Hz})$. Participants were then exposed to one of the four conditioning protocols. Participants were blind concerning the tDCS polarity condition (anodal or cathodal) or PAS timing (PAS10 or PAS25). Immediately after conditioning, a second set of 25 MEPs was obtained with TMS. This procedure was repeated every 5 minutes for the first 30 minutes post-conditioning, and then every $30 \mathrm{~min}$ for the next one and a half hours. The participants returned for an additional block of TMS trials that evening, and three times during the next day (morning, noon, and evening). In sum, motor cortex excitability was probed in 14 epochs after tDCS or PAS conditioning.

For participants who completed more than one conditioning protocol, a minimum of seven days separated successive protocols. For these participants, the intensity of TMS stimulation was adjusted at the beginning of each session and the order of conditioning type was randomized.

\section{Data analysis}

The goal of this study was to determine if individual differences in the efficacy of tDCS can, in part, be explained by individual differences in sensitivity to TMS. We looked at this question using a median split procedure in which we divided the participants within each conditioning protocol into two groups, Low and High TMS Intensity. The Low Intensity group was composed of individuals requiring lower TMS stimulation levels (MSO) to produce 1 mV MEPs at baseline; the High Intensity group was composed of 
individuals requiring higher TMS stimulation level to produce 1 mV MEPs.

The data were visually inspected to exclude trials in which there was significant background EMG activity greater than $0.01 \mathrm{mV}$ in the $200 \mathrm{~ms}$ window preceding the TMS pulse $[25,26]$. We also removed MEPs outliers, defined by those in which the amplitude was $\pm 2 \mathrm{sd}$ of the mean MEPs (for each condition).

MEPs were averaged within each of the 14 epochs. We first evaluated the normality of the data for each epoch with the KolmogorovSmirnov test (using the residuals of the raw data). The average MEP values for each epoch were then normalized with respect to baseline on an individual basis, with values greater than 1 indicating an increase in excitability, and values smaller than 1 an excitability reduction. For epochs in which the data were normally distributed, we used a series of t-tests to examine if a conditioning protocol produced a significant change in MEPs amplitude, relative to baseline.

The data were pooled to create three time windows: Early (0-30 min), Middle (60-120 min), and Late (evening and next day). Given that MEPs in all four protocols had returned to baseline in the Late window, we restricted this analysis to the Early and Middle windows. Separate ANOVAs were conducted for each conditioning protocol, with one between-subject factor (Group: Low Intensity vs. High Intensity) and a within-subject repeating factor (Time: Early vs. Middle epoch). Given that there were some violations of normality, we supplemented the ANOVA with nonparametric permutation statistics (see Results section).

Correlation coefficients were calculated for the two tDCS protocols, with one variable being the TMS stimulation level and the other being the average normalized MEP value for the Early epoch. With this analysis, variation in TMS intensity was treated as a continuous variable rather than being categorically divided into Low and High Intensity groups.

\section{Results}

Individual differences in TMS intensity

Participants were divided into two groups based on the stimulation level required to produce $1 \mathrm{mV}$ MEPs. The median MSO was similar for all four conditioning protocols. For each protocol, participants with values lower than the median MSO were assigned to the Low Intensity group and participants with values higher than the median MSO were assigned to the High Intensity group. For anodal tDCS, the median MSO was 49.0 (Low: $\mathrm{n}=17$, MSO range: 30-48; High: $\mathrm{n}=19$, MSO range: 49-69). For cathodal tDCS the median MSO was 47.5 (Low: $n=17$, range: $32-47$; High: $n=17$, range: 48-68). For PAS25, the median MSO was 48.5 (Low: $n=18$, range: 31-48; High: $\mathrm{n}=18$, range: 49-67). For PAS10, the median MSO was 47.5 (Low: $\mathrm{n}=18,34-47$; High: $\mathrm{n}=18$, range: $47-67$ ).

\section{Efficacy of the conditioning protocols}

As assessed by Kolmogorov-Smirnov test, the MEPs data in the Anodal condition met the criteria for normality in 13 of the 14 epochs, with the one violation at $15 \mathrm{~min}$. However, more frequent violations of normality were observed in the other three conditioning protocols. The cathodal data were not normally distributed for four epochs (t25, t90, t120, next day noon). For the PAS protocol, violations were observed in four epochs for the PAS10 condition (15 min, 20 min, 25 min, same evening) and six epochs in the PAS25 condition ( $5 \mathrm{~min}, 10 \mathrm{~min}, 15 \mathrm{~min}, 20,120 \mathrm{~min}$, next evening). Given this mixed picture, we present both parametric and non-parametric statistics in our evaluation of the effects of the conditioning protocols.

Consistent with previous reports, all conditioning protocols led to measureable changes in corticospinal excitability (Fig. 2). Relative to baseline, anodal stimulation and PAS25 produced an increase in MEPs, whereas cathodal stimulation and PAS10 decreased MEPs. The change from baseline was significant (all $<0.05$, analysis restricted to epochs that did not violate test of normality) for all four conditioning protocols for up to $90 \mathrm{~min}$ after conditioning. At $120 \mathrm{~min}$, the MEPs were indistinguishable from baseline for tDCS, while remained significant for PAS. No persistent changes were observed on the evening following conditioning, or on the subsequent day.

Modulation of conditioning effects due to individual differences in TMS intensity

To examine if variation in sensitivity to TMS influenced the efficacy of the conditioning protocols, we compared the dynamics of the MEPs changes for participants in the Low and High Intensity

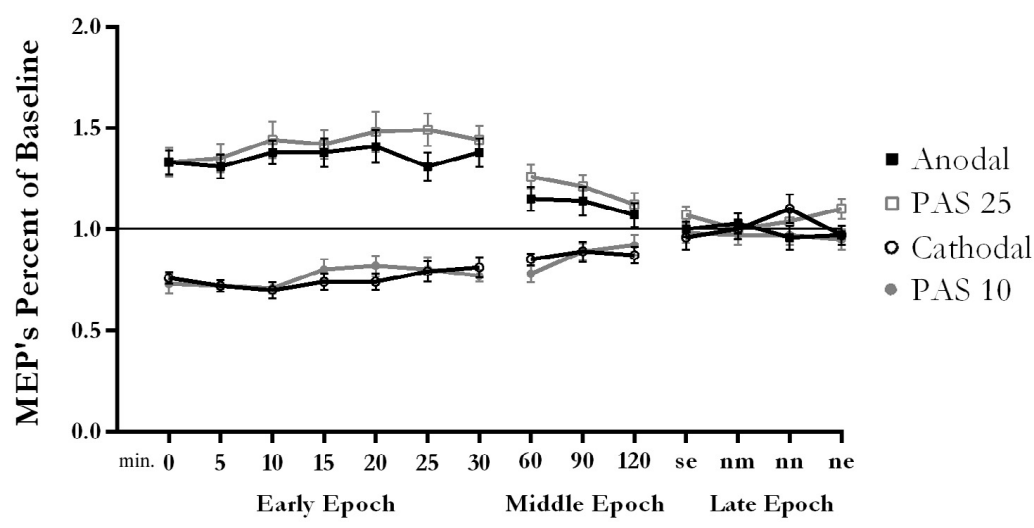

Time After tDCS

Figure 2. MEPs changes at each epoch for the four conditioning protocols. The data are averaged over all participants for a given condition. In black are shown MEPs changes after tDCS conditioning protocols (anodal filled square, cathodal empty circle) and in gray are shown MEPs changes after PAS conditioning protocols (PAS 25 empty square, PAS 10 full circle). Error bars indicate SEMs. 


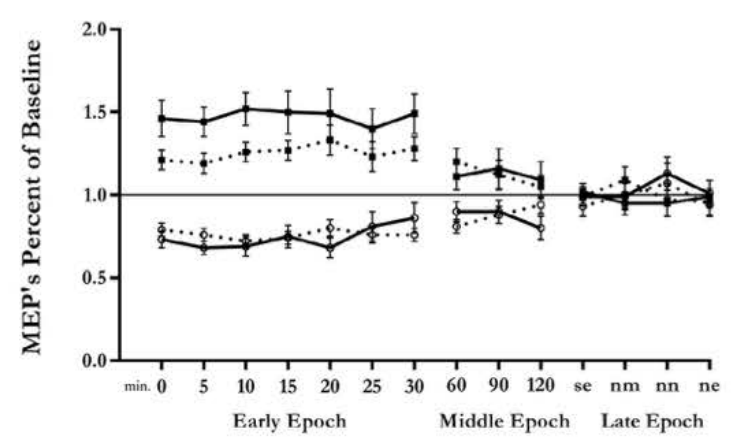

Time After tDCS

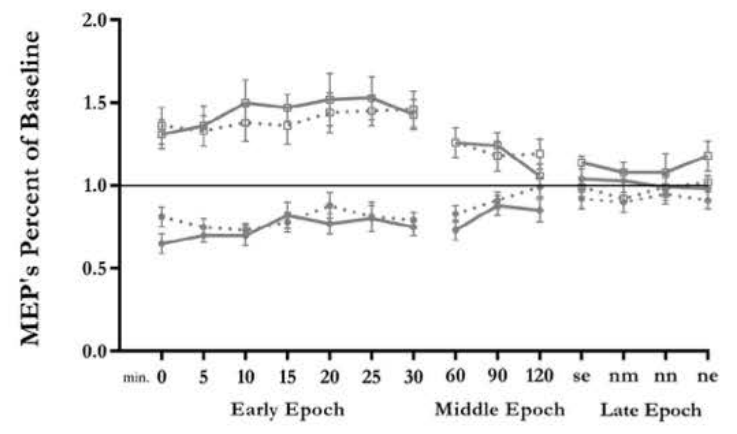

Time After tDCS a

$\rightarrow$ Anodal Low

... Anodal High

$\rightarrow$ Cathodal Low

... Cathodal High

- PAS 25 Low

*. PAS 25 High

- PAS 10 Low

... PAS 10 High

Figure 3. MEPs changes at each epoch for the tDCS (a) and PAS (b) conditioning protocols, with the participants in each condition divided into Low Intensity (filled lines) and High Intensity groups (dotted lines). The division was based on a median split defined by the level of TMS stimulation required to elicit $1 \mathrm{mV}$ MEPs prior to the conditioning protocol. Error bars indicate SEMs.

groups. The effects of conditioning remain relatively constant for the first $30 \mathrm{~min}$ and then decrease over the remaining epochs in the initial 2-hour session (Fig. 3). Given this, we pooled the MEPs data into two time periods, Early (0-30 min) and Middle (60-120 min), excluding the other epochs since there was no residual effect of the conditioning protocols. For anodal stimulation, the main effect of Time, $\mathrm{F}(1,34)=20.69, \mathrm{p}<0.001$, but not of Group, $\mathrm{F}(1,34)=1.59$, $\mathrm{p}=0.216$, was significant. However, the interaction of these two factors was significant, $F(1,34)=4.44, p=0.043$. As can be seen in Figs. $3 \mathrm{a}$ and 4 , anodal tDCS produced a larger increase in MEPs in the Low Intensity group, but this effect was limited to the early time window (Early: $\mathrm{t}=2.08, \mathrm{p}=0.045$; Middle: $\mathrm{t}=-0.32, \mathrm{p}=0.98$ ). This result is consistent with the hypothesis that individual differences in sensitivity to TMS impact the efficacy of anodal tDCS. Contrary to our expectations, this pattern was not observed with cathodal tDCS. Here we observed a main effect of Time, $(F(1,32)=11.241$, $\mathrm{p}=0.002)$, but no effect of group $(\mathrm{F}(1,32)<1.0)$ nor an interaction $(\mathrm{F}(1,32)<1.0)$.

Individual differences in TMS intensity did not influence the efficacy of PAS (Fig. 3b). For both PAS10 and PAS25, there was a significant effect of Time (all p's $<0.03$ ), but not of Group and the respective interactions (all p's $>0.20$ ). The null effects here are in line with expectations given that, for the PAS protocols, TMS intensity has been adjusted individually.

The violations of normality in some of the epochs are unlikely to have had a major impact on the ANOVAs. First, we pooled the data across epochs to obtain more robust samples for each individual.
Second, violations of normality increase the likelihood of a false positive result, although simulation studies have shown that this increase is modest for moderate deviations from normality [27-29]. We did not obtain significant Group or interaction effects for the three

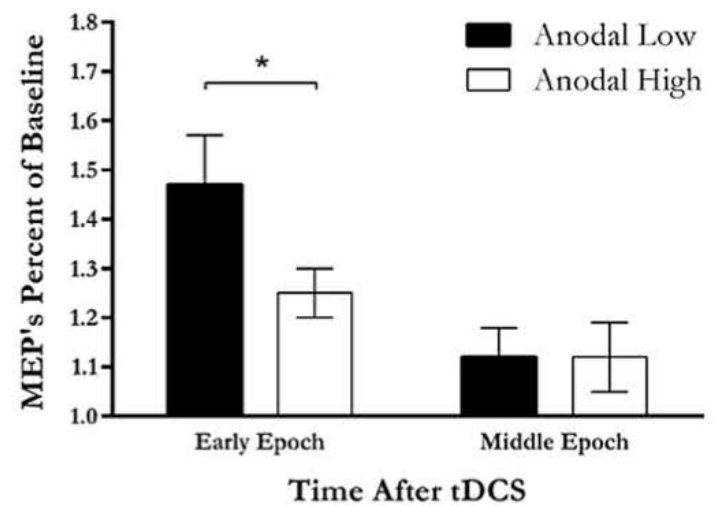

Figure 4. MEPs changes in the Early and Middle time windows for participants in the anodal tDCS protocol, with the participants divided into Low Intensity (black filling) and High Intensity (white filling) groups. Error bars indicate SEMs. 
individuals requiring higher TMS stimulation level to produce 1 mV MEPs.

The data were visually inspected to exclude trials in which there was significant background EMG activity greater than $0.01 \mathrm{mV}$ in the $200 \mathrm{~ms}$ window preceding the TMS pulse $[25,26]$. We also removed MEPs outliers, defined by those in which the amplitude was \pm 2 sd of the mean MEPs (for each condition).

MEPs were averaged within each of the 14 epochs. We first evaluated the normality of the data for each epoch with the KolmogorovSmirnov test (using the residuals of the raw data). The average MEP values for each epoch were then normalized with respect to baseline on an individual basis, with values greater than 1 indicating an increase in excitability, and values smaller than 1 an excitability reduction. For epochs in which the data were normally distributed we used a series of $t$-tests to examine if a conditioning protocol produced a significant change in MEPs amplitude, relative to baseline

The data were pooled to create three time windows: Early (0-30 min), Middle (60-120 min), and Late (evening and next day). Given that MEPs in all four protocols had returned to baseline in the Late window, we restricted this analysis to the Early and Middle windows. Separate ANOVAs were conducted for each conditioning protocol, with one between-subject factor (Group: Low Intensity vs. High Intensity) and a within-subject repeating factor (Time: Early vs. Middle epoch). Given that there were some violations of normality, we supplemented the ANOVA with nonparametric permutation statistics (see Results section).

Correlation coefficients were calculated for the two tDCS protocols, with one variable being the TMS stimulation level and the other being the average normalized MEP value for the Early epoch. With this analysis, variation in TMS intensity was treated as a continuous variable rather than being categorically divided into Low and High Intensity groups.

\section{Results}

Individual differences in TMS intensity

Participants were divided into two groups based on the stimulation level required to produce $1 \mathrm{mV}$ MEPs. The median MSO wa similar for all four conditioning protocols. For each protocol, participants with values lower than the median MSO were assigned to the Low Intensity group and participants with values higher than the median MSO were assigned to the High Intensity group. For anodal $\mathrm{tDCS}$, the median MSO was 49.0 (Low: $n=17$, MSO range: 30-48; High: $n=19$, MSO range: 49-69). For cathodal tDCS the median MSO was 47.5 (Low: $n=17$, range: $32-47$; High: $n=17$, range: 48-68). For PAS25, the median MSO was 48.5 (Low: $n=18$, range: 31-48; High: $n=18$, range: 49-67). For PAS10, the median MSO was 47.5 (Low: $n=18,34-47$; High: $n=18$, range: $47-67$ ).

\section{Efficacy of the conditioning protocols}

As assessed by Kolmogorov-Smirnov test, the MEPs data in the Anodal condition met the criteria for normality in 13 of the 14 epochs, with the one violation at $15 \mathrm{~min}$. However, more frequent violations of normality were observed in the other three conditioning protocols. The cathodal data were not normally distributed for four epochs ( $t 25, t 90, t 120$, next day noon). For the PAS protocol, violations were observed in four epochs for the PAS10 condition (15 min, $20 \mathrm{~min}, 25 \mathrm{~min}$, same evening) and six epochs in the PAS25 condition ( $5 \mathrm{~min}, 10 \mathrm{~min}, 15 \mathrm{~min}, 20,120 \mathrm{~min}$, next evening). Given this mixed picture, we present both parametric and non-parametric statistics in our evaluation of the effects of the conditioning protocols.

Consistent with previous reports, all conditioning protocols led to measureable changes in corticospinal excitability (Fig. 2). Relative to baseline, anodal stimulation and PAS25 produced an increase in MEPs, whereas cathodal stimulation and PAS10 decreased MEPs. The change from baseline was significant (all $<0.05$, analysis restricted to epochs that did not violate test of normality) for all four conditioning protocols for up to $90 \mathrm{~min}$ after conditioning. At $120 \mathrm{~min}$, the MEPs were indistinguishable from baseline for tDCS, while remained significant for PAS. No persistent changes were observed on the evening following conditioning, or on the subsequent day.

Modulation of conditioning effects due to individual differences in TMS intensity

To examine if variation in sensitivity to TMS influenced the efficacy of the conditioning protocols, we compared the dynamics of the MEPs changes for participants in the Low and High Intensity

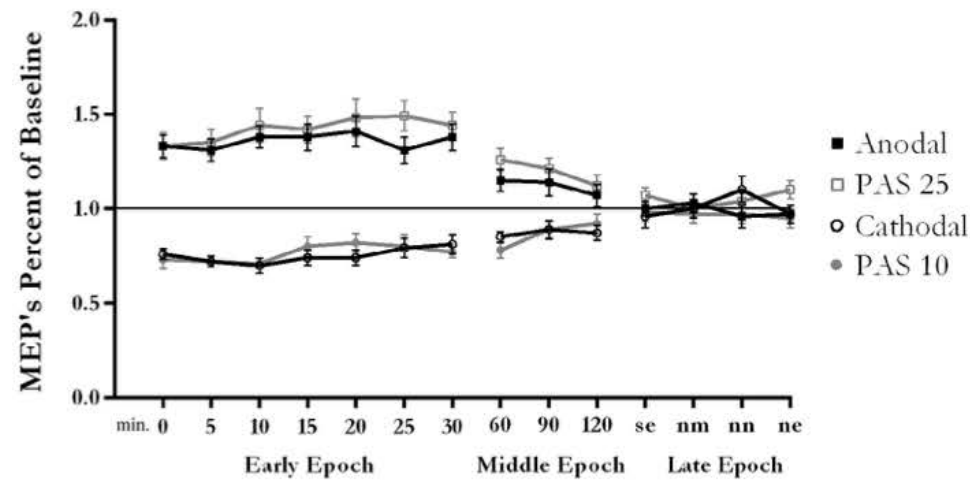

Time After tDCS

Figure 2. MEPs changes at each epoch for the four conditioning protocols. The data are averaged over all participants for a given condition. In black are shown MEPs changes after tDCS conditioning protocols (anodal filled square, cathodal empty circle) and in gray are shown MEPs changes after PAS conditioning protocols (PAS 25 empty square, PAS 10 full circle). Error bars indicate SEMs. 
Anodal

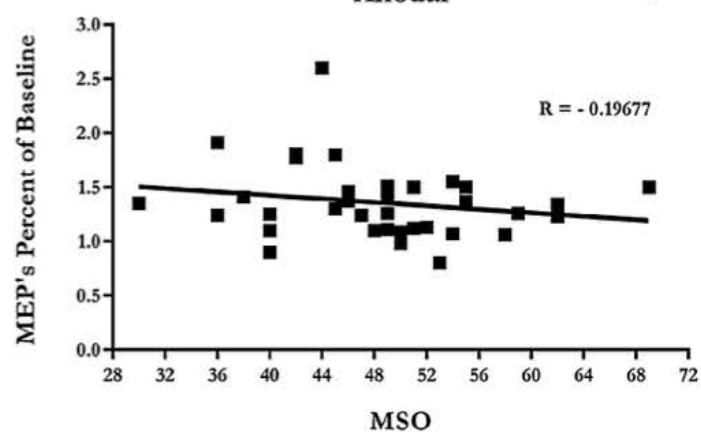

Cathodal

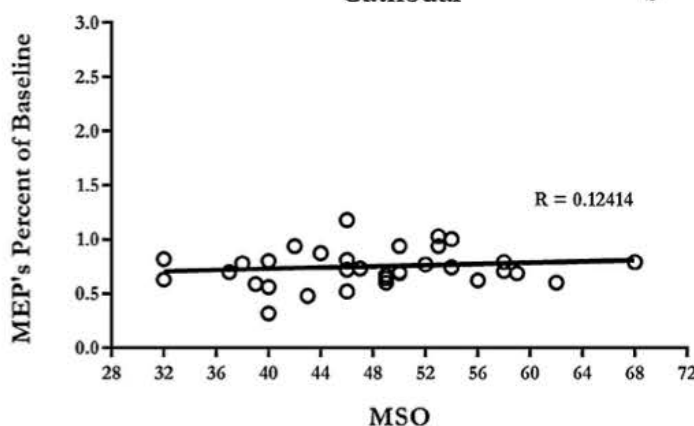

Figure 5. Correlation between intensity of TMS stimulation and averaged normalized MEP value. Data are from the Early time window ( $0-30$ min after intervention) for the anodal (a) and cathodal (b) tDCS groups.

conditioning protocols in which there were substantial violations. Third, the normality criterion was generally met for the anodal group, the one protocol showing the Group $\times$ Time interaction. However given the violations of the normality assumption, we also applied nonparametric permutation statistics to assess the MEPs data, comparing the Low and High Intensity groups in the Early and Middle time windows for the different conditioning protocols. Randomization tests were conducted in which individuals were randomly assigned, with replacement, to one of two groups to create a distribution of the expected differences ( 10,000 permutations). From this distribution, we calculated the p value for our observed values. Consistent with the parametric analyses, the difference between the Low and High Intensity groups for the anodal group was significant in the Early epoch $(p=0.021)$. There was no effect of group for the other conditioning protocols in the early window, nor an effect of group for any of the conditioning protocols in the Middle epoch (all p's $>0.35$ ).

We also explored the data in a continuous manner, correlating stimulation intensity with the post-conditioning change in MEPs (Fig. 5). In the Early epoch there was a negative correlation for the anodal group $(r=-0.197, p=0.125)$ and a positive correlation for the cathodal group $(r=0.124, p=0.241)$. Although neither correlation was significant, both are in the predicted direction if the efficacy of tDCS conditioning is related to the TMS stimulation level. We note that one participant had a much larger MEP (MSO 44/MEP 2.60) than the group, and another a much larger MSO (MSO 69/MEP 1.50), raising concerns that these correlations might be driven by outliers.
However, the correlations remain unaffected when redone without these individuals. Dropping the large MEP participant reduced the correlation to -0.18 ; dropping the large MSO participant increased the correlation to -0.25 . Given that neither value was more than $2.5 \mathrm{SD}$ from the average, we have opted to include all of the data.

\section{Discussion}

The data presented in this archival analysis demonstrate that the efficacy of tDCS in inducing changes in corticospinal excitability varies as a function of individual differences in the sensitivity to TMS. Individuals requiring a lower TMS stimulation level to produce a criterion MEP amplitude size showed a larger change in MEPs following anodal tDCS, compared to individuals requiring a higher TMS stimulation intensity. This effect was not found for cathodal tDCS. Given the widespread use of anodal tDCS to modulate motor and cognitive functions, these results highlight a potentially relevant covariate to consider when evaluating the efficacy tDCS. It should be taken into account that the TMS intensities used in this analysis to define low and high intensity groups should not be taken as absolute values given the variation in output delivered by the TMS device from different manufacturers.

Individual sensitivity to TMS is widely recognized as a critical factor in the TMS literature [30]: indeed the standard protocol in the field entails the use of a pre-experiment phase to "equate" the physiological/functional stimulation level across individuals. This procedure, regardless of whether the criterion involves establishing resting threshold or a target MEP size, always reveals substantial variation. For example, the stimulation level required to produce a $1 \mathrm{mV}$ MEP varied from $30 \%$ to $69 \%$ of the maximum output of the stimulator in the current data set. Although TMS and tDCS operate under different mechanisms to influence cortical physiology, anatomical and physiological properties that influence the efficacy of stimulation might be similar within an individual. Relevant factors would include skull thickness, overall brain shape, the pattern of cortical folding, receptor distribution, transmitter and neuromodulator availability.

Methodologically, researchers have not considered individual variation in sensitivity to brain stimulation as a means to adapt stimulation protocols for tDCS studies. Rather, the convention has been to employ a fixed conditioning protocol for all individuals. The results presented here suggest that using a fixed stimulation level may negatively impact the robustness of tDCS research given that one source of variability, individual sensitivity to tDCS, is not controlled. The importance of this issue is evident in recent discussions on the efficacy and reliability of tDCS [13-16,31]. We suggest that some of this variability may arise from the failure to consider individual differences in the sensitivity to tDCS.

The relationship between sensitivity to TMS and the efficacy of tDCS appears to be modest. While the group effect is substantial in our median split analysis, when the data were treated in a continuous manner, the correlation between our two measures was only -0.20 . By conventional estimates, this would mean that differences in sensitivity account for only $4 \%$ of the variance. We note that this estimate represents the lower limit given that correlations are limited by the reliability in the measurement of each variable; one can assume that reliability is lower at the individual level compared to the group analysis. Nonetheless, there are many reasons to expect limitations in the relationship between TMS and tDCS. While individual variation in anatomy or neurotransmitter concentrations should have similar effects on TMS and tDCS, other variables such as hair thickness and skin conductivity impact the efficacy of tDCS, but not TMS. 
Factors underlying individual differences in the efficacy of tDCS have been considered in some studies. Opitz et al. [32] used a model of the head to investigate how anatomical features shape the electric field distribution in the brain during tDCS. They showed that individual characteristics, such as the thicknesses of the skull and sulcal depth, influence electrical field distribution. Taking a similar approach, Kim et al. [33] showed that a composite of anatomical features based on individual MRIs was related to behavioral changes in working memory.

To date, only a few studies have directly examined individual responsiveness to TMS and anodal tDCS [34]. López-Alonso et al. [35] used a cluster analysis to test whether baseline TMS measures (e.g., resting motor threshold and stimulation level required to produce $1 \mathrm{mV}$ MEPs) were correlated with the efficacy of tDCS in modulating corticospinal excitability. This analysis failed to reveal a relationship between responsiveness to the TMS and tDCS measures. This result stands in contrast to our findings. However, the authors do not report the range of stimulation levels employed in the study, making it difficult to compare with our study. In addition, a large percentage of the participants (45\%) failed to show enhanced MEPs following anodal tDCS (non-responders group) while in our pool of subjects only 3 subjects out of 38 were non-responders (8\%).

We also note that baseline MEPs amplitudes differed between clusters in the López-Alonso et al. [35] study. The non-responders group had significantly higher MEPs compared to the responders group. One possibility is that the lower baseline MEPs in the responders may have afforded greater sensitivity to observe an increase in excitability after tDCS, a hypothesis consistent with the findings of Wiethoff et al. [14]. This factor is unlikely to influence our results given that baseline MEPs were similar across all groups. For example, in our median split, baseline MEPs amplitudes were close to the targeted $1.0 \mathrm{mV}$ level and did not differ between the High and Low Intensity groups (mean $\pm S D$, Low Intensity group: $1.07 \pm 0.10$; High Intensity group: $1.05 \pm 0.14 ; \mathrm{t}=0.35, \mathrm{p}=0.72$ ).

We did not observe a relationship between sensitivity to TMS and the efficacy of cathodal tDCS. Although cathodal tDCS reduced corticospinal excitability for an extended period of time, the effect was comparable for the Low and High Intensity groups. At present, we can only speculate about why the relationship observed with anodal tDCS was not observed with cathodal tDCS. It may be that the range of responsiveness to cathodal tDCS is more restricted than that of anodal tDCS. Alternatively, our results might have been influenced by the fact that the impact of cathodal tDCS on neuroplasticity is non-linear and that there is no attenuation of excitability following cathodal tDCS if the stimulation level is too strong or too weak $[7,34]$. Our fixed tDCS conditioning intensity of $1 \mathrm{~mA}$ may have been functional ineffective for participants who were least or most sensitive to TMS. Future studies that systematically vary stimulation intensity will be required to assess these hypotheses.

We also did not observe differences in our two groups in terms of the efficacy of the PAS conditioning protocols. This null result was predicted given that with PAS, the stimulation level is individually adjusted for both TMS and electrical stimulation [10]. However, Müller-Dahlhaus et al. [36] reported that resting motor threshold and stimulation levels based on a $1 \mathrm{mV}$ criterion are negatively correlated with PAS20 +2 , another PAS protocol thought to produce an increase of corticospinal excitability. It is difficult to relate these results to our findings given that the PAS20 +2 protocol in the MüllerDahlhaus study produced inconsistent changes in corticospinal excitability. About half of their participants (52\%) showed an increase in MEPs amplitude following PAS20 + 2, with the rest showing no change or a decrease in excitability. In our study, 31 of the 36 participants (86\%) showed an increase in excitability with the PAS25 protocol.
In summary, our results demonstrate a relationship between individual differences in sensitivity to TMS and the efficacy of anodal tDCS. The nature of this relationship remains to be explored in greater detail: it may or may not be linear, and there remains the puzzle of understanding why the effect was limited to anodal tDCS. Moreover in the current study, the stimulation intensity for TMS was set to produce MEPs of $1 \mathrm{mV}$. We opted to use this measure since our archival data sets had a large number of participants who had received tDCS or PAS with this TMS criterion. Future studies need to investigate how the relationship between TMS sensitivity and tDCS efficacy holds for different measures of TMS sensitivity (e.g., resting motor threshold). More generally, it will be important to establish if individual differences in TMS sensitivity remain stable across the recruitment curve. Future studies will also need to address how the individual characteristics influence the efficacy of tDCS in multiple sessions, given that this has been reported to be the an efficient way to induce robust changes in healthy controls [37] and in patients [38,39]. It will also be important to consider if individual variation in sensitivity assessed over the motor cortex is relevant when considering the efficacy of tDCS targeted at other brain regions. Another approach to consider would be to obtain MEPs during tDCS, placing the TMS coil over the M1 electrode [40]. Indeed, as seen in Fig. 3, the difference between the Low and High groups is evident at our first sample, obtained just after the end of the tDCS stimuation phase. Using TMS during tDCS would allow us to see the emergence of this difference.

Of practical relevance, our results suggest an interesting direction for research designed to improve the efficacy of tDCS. Similar to standard practices in TMS research, practitioners of tDCS should consider methods to "equate", physiologically and functionally, the stimulation level for tDCS. Given that tDCS does not produce an overt physiological response (e.g., MEPs), we propose that the stimulation level for tDCS could be adjusted on an individual basis by extrapolating from individual variation in sensitivity to TMS.

\section{Acknowledgements}

MAN was supported by a grant from the German Ministry for Education and Research (03IPT605E), and the DFG (grants NI 683/ 4-1, NI 683/4-2, NI 683/6-1). RBI was supported by a grant from the NIH (NS074917).

\section{References}

[1] Stefan K, Kunesch E, Cohen LG, Benecke R, Classen J. Induction of plasticity in the human motor cortex by paired associative stimulation. Br J Neurol the human motor cor 123 (Pt 3):572-84.

[2] Galea JM, Vazquez A, Pasricha N, de Xivry J-JO, Celnik P. Dissociating the roles of the cerebellum and motor cortex during adaptive learning: the motor cortex retains what the cerebellum

3) Dayan E, Censor N, Buch ER, Sandrini M, Cohen LG. Noninyasive brain stimulation: from physiology to network dynamics and back. Nat Neurosci 2013:16:838-44. doi:10.1038/nn.3422.

[4] Ferrucci R, Priori A. Transcranial cerebellar direct current stimulation (tcDCS): motor control, cognition, learning and emotions. Neuroimage 2014:85(Pt 3):918-23. doi:10.1016/j.neuroimage.2013.04.122.

[5] Kuo M-F, Paulus W, Nitsche MA. Therapeutic effects of non-invasive brain stimulation with direct currents ( $t D C S)$ in neuropsychiatric diseases. Neuroimage 2014;85(Pt 3):948-60. doi:10.1016/j.neuroimage.2013.05.117.

[6] Vallence A-M, Ridding MC. Non-invasive induction of plasticity in the human cortex: uses and limitations. Cortex J Devoted Study Nerv Syst Behav 2013; doi:10.1016/j.cortex.2013.12.006

7] Nitsche MA, Paulus W. Excitability changes induced in the human motor cortex by weak transcranial direct current stimulation.J Physiol 2000;527(Pt 3):633-9. 8] Carson RG, Kennedy NC. Modulation of human corticospinal excitability by paired associative stin

9] Rossi S, Hallett M, Rossini PM, Pascual-Leone A. Safety, ethical considerations, and application guidelines for the use of transcranial magnetic stimulation in 
clinical practice and research. Clin Neurophysiol Off J Int Fed Clin Neurophysio 2009;120:2008-39. doi:10.1016/j.clinph.2009.08.016.

[10] Ziemann U, Ilić TV, lliać TV, Pauli C, Meintzschel F, Ruge D. Learning modifie subsequent induction of long-term potentiation-like and long-term depressionlike plasticity in human motor cortex. J Neurosci Off J Soc Neurosc 2004;24:1666-72. doi:10.1523/NEUROSC1.5016-03.2004

[11] Opitz A, Legon W, Rowlands A, Bickel WK, Paulus W, Tyler W]. Physiologica observations validate finite element models for estimating subject-specific electric field distributions induced by transcranial magnetic stimulation of the human motor cortex. Neuroimage 2013:81:253-64. doi:10.1016 j.neuroimage.2013.04.067.

[12] Rossini PM, Barker AT, Berardelli A, Caramia MD, Caruso G, Cracco RQ et al. Non-invasive electrical and magnetic stimulation of the brain, spinal cord an roots: basic principles and procedures for routine clinical application. Report

131 Horvath JC. Carter 0, Forte JD. Transcranial direct corrent stimulation: five important issues we aren't discussing (but probably should be). Front Syst Neurosci 2014;8:2. doi:10.3389/fnsys.2014.00002.

14] Wiethoff S, Hamada M. Rothwell JC Variability in response to transcranial direct current stimulation of the motor cortex. Brain Stimulat 2014:7:468-75. doi:10.1016/j.brs.2014.02.003.

[15] Price AR, Hamilton RH. A re-evaluation of the cognitive effects from single-session transcranial direct current stimulation. Brain Stimulat 2015; doi: $10.1016 / \mathrm{j}$. brs.2015.03.007

[16] Horvath JC, Forte JD, Carter O. Quantitative review finds no evidence of cognitive effects in healthy populations from single-session transcranial direct current stimulation (tDCS). Brain Stimulat 2015; doi:10.1016/j.brs.2015.01.400

[17] Batsikadze G, Paulus W, Grundey J, Kuo M-F, Nitsche MA. Effect of the nicotinic $\alpha 4 \beta 2$-receptor partial agonist varenicline on non-invasive brain stimulationinduced neuroplasticity in the human motor cortex. Cereb Cortex N Y N 1991;2014:doi:10.1093/cercor/bhu12

[18] Fresnoza S, Paulus W, Nitsche MA, Kuo M-F. Nonlinear dose-dependent impact of D1 receptor activation on motor cortex plasticity in humans. J Neurosci

[19] Fresnoza S, Stiksrud E, Klinker F, Liebetanz D, Paulus W, Kuo M-F, et al. Dosage-dependent effect of dopamine d2 receptor activation on motor cortex plasticity in humans. J Neurosci Off J Soc Neurosci 2014;34:10701-9. doi:10.1523/JNEUROSCI.0832-14.2014

[20] Oldfield RC. The assessment and analysis of handedness: the Edinburgh inventory. Neuropsychologia 1971;9:97-113.

21] Nitsche MA, Paulus W. Sustained excitability elevations induced by transcrania DC motor cortex stimulation in humans. Neurology 2001:57:1899-901. doi: $10.1212 /$ WNL.57.10.1899

[22] Nitsche MA, Fricke K, Henschke U, Schlitterlau A, Liebetanz D, Lang N, et al. Pharmacological modulation of cortical excitability shifts induced by transcranial direct current stimulation in humans. J Physiol 2003;553:293-301. doi:10.1113/ jphysiol.2003.049916.

[23] Wolters A, Sandbrink F, Schlottmann A, Kunesch E, Stefan K, Cohen LG, et al. A temporally asymmetric Hebbian rule governing plasticity in the human motor

Cortex.J Neurophysiol 2003;89:2339-45. doi:10.1152/jn.00900.2002.
[24] Stefan K, Kunesch E, Benecke R, Cohen LG, Classen J. Mechanisms of enhancement of human motor cortex excitability induced by interventional paired associative stimulation. J Physiol 2002;543:699-708. [25] Duque J, Mazzocchio R, Dambrosia J, Murase N, Olivier E, Cohen LG. generation of a voluntary movement. Cereb Cortex N Y N 1991 2005;15:588-93. doi:10.1093/cercor/bhh160.

[26] Duque J, Murase N, Celnik P, Hummel F, Harris-Love M, Mazzocchio R, et al. Intermanual differences in movement-related interhemispheric inhibition. J Cogn Neurosci 2007; 19:204-13. doi:10.1162/jocn.2007.19.2.204.

[27] Glass GV, Peckham PD, Sanders JR. Consequences of failure to meet assumptions underlying the fixed effects analyses of variance and covariance. Rev Educ Res 1972;42:237-88. doi:10.2307/1169991.

[28] Harwell MR, Rubinstein EN, Hayes WS, Olds CC. Summarizing Monte Carlo results in methodological research: the one- and two-factor fixed effects ANOVA cases. J Educ

[29] Lix LM, Keselman JC, Keselman HJ. Consequences of assumption violations revisited: a quantitative review of alternatives to the one-way analysis

[30] Wassermann EM. Inter- and intra-individual variation in the response to TMS, in Oxford Handbook of Transanial Stimulation Oxford University Press: 2008.

[31] Nitsche MA, Bikson M, Bestmann S. On the use of meta-analysis in neuromodulatory non-invasive Brain stimulation. Brain Stimul Basic Transl Clin Res Neuromodulation 2015;0:doi:10.1016/j.brs.2015.03.008.

[32] Opitz A, Paulus W, Will S. Antunes A, Thielscher A. Determinants of the electric field during transcranial direct current stimulation. Neuroimage 2015;109:14050. doi:10.1016/j.neuroimage.2015.01.033.

[33] Kim J-H, Kim D-W, Chang WH, Kim Y-H, Kim K, Im C-H. Inconsistent outcomes of transcranial direct current stimulation may originate from anatomical differences among individuals: electric field simulation using individual MRI data. Neurosci Lett 2014;564:6-10. doi:10.1016/j.neulet.2014.01.054.

[34] Batsikadze G, Moliadze V, Paulus W, Kuo M-F, Nitsche MA. Partially non-linear stimulation intensity-dependent effects of direct current stimulation on motor cortex excitability in humans. J Physiol 2013;591:1987-2000. doi:10.1113/

jphysiol.2012.249730.
[35] López-Alonso V, Cheeran B, Río-Rodríguez D, Fernández-Del-Olmo M. Interindividual variability in response to non-invasive brain stimulation paradigms.

Brain Stimulat 2014; doi:10.1016/j.brs.2014.02.004.
[36] Müller-Dahlhaus JFM, Orekhov Y, Liu Y, Ziemann U. Interindividual variability and age-dependency of motor cortical plasticity induced by paired associative

[37] Reis J Schambra HM, Cohen LG, Buch ER Fritsch B, Zarahn E, et at. Noninvasive cortical stimas an effect on consolidation. Proc Natl Acad Sci 2009;106:1590-5. doi:10.1073/ pnas.0805413106.

[38] Boggio PS, Nunes A, Rigonatti SP, Nitsche MA, Pascual-Leone A, Fregni F. Repeated sessions of noninvasive brain DC stimulation is associated with motor 9 .

[39] Boggio PS, Rigonatti SP, Ribeiro RB, Myczkowski ML, Nitsche MA, Pascual-Leone A, et al. A randomized, double-blind clinical trial on the efficacy of cortical direct current stimulation for the treatment of major depression. Int ] Neuropsychopharmacol Off Sci Coll Int Neuropsychopharmacol CINP 2008;11:249-54. doi:10.1017/S1461145707007833.

[40] Moliadze V, Antal A, Paulus W. Electrode-distance dependent after-effects of transcranial direct and random noise stimulation with extracephalic reference electrodes. Clin Neurophysiol Off J Int Fed Clin Neurophysiol 2010;121:2165-71. 


\section{Systematic evaluation of the impact of stimulation intensity on neuroplastic after-effects induced by transcranial direct current stimulation}

\section{Systematic evaluation of the impact of stimulation intensity on neuroplastic after-effects induced by transcranial direct current stimulation}

Asif Jamil ${ }^{1,2}$, Giorgi Batsikadze ${ }^{1}$, Hsiao-I. Kuo ${ }^{1,2}$, Ludovica Labruna ${ }^{3}$, Alkomiet Hasan $^{4}$, Walter Paulus ${ }^{1}$ and Michael A. Nitsche $e^{2,5}$

${ }^{1}$ Department of Clinical Neurophysiology, University Medical Center, University of Göttingen, 37075 Göttingen, Germany

${ }^{2}$ Leibniz Research Centre for Working Environment and Human Factors, 44139 Dortmund, Germany

${ }^{3}$ Department of Psychology, University of California, Berkeley, CA 94720, USA

${ }^{4}$ Department of Psychiatry and Psychotherapy, Klinikum der Universität München, 80336 München, Germany

${ }^{5}$ University Medical Hospital Bergmannsheil, 44789 Bochum, Germany

Key points

- Applications of transcranial direct current stimulation to modulate human neuroplasticity have increased in research and clinical settings.

- However, the need for longer-lasting effects, combined with marked inter-individual variability, necessitates a deeper understanding of the relationship between stimulation parameters and physiological effects.

- We systematically investigated the full DC intensity range $(0.5-2.0 \mathrm{~mA})$ for both anodal and cathodal tDCS in a sham-controlled repeated measures design, monitoring changes in motor-cortical excitability via transcranial magnetic stimulation up to $2 \mathrm{~h}$ after stimulation.

- For both tDCS polarities, the excitability after-effects did not linearly correlate with increasing DC intensity; effects of lower intensities $(0.5,1.0 \mathrm{~mA})$ showed equal, if not greater effects in motor-cortical excitability.

- Further, while intra-individual responses showed good reliability, inter-individual sensitivity to TMS accounted for a modest percentage of the variance in the early after-effects of $1.0 \mathrm{~mA}$ anodal tDCS, which may be of practical relevance for future optimizations.

Abstract Contemporary non-invasive neuromodulatory techniques, such as transcranial direct current stimulation (tDCS), have shown promising potential in both restituting impairments in cortical physiology in clinical settings, as well as modulating cognitive abilities in the healthy population. However, neuroplastic after-effects of tDCS are highly dependent on stimulation parameters, relatively short lasting, and not expectedly uniform between individuals. The present study systematically investigates the full range of current intensity between 0.5 and $2.0 \mathrm{~mA}$ on left primary motor cortex (M1) plasticity, as well as the impact of individual-level covariates on explaining inter-individual variability. Thirty-eight healthy subjects were divided into groups of anodal and cathodal tDCS. Five DC intensities (sham, $0.5,1.0,1.5$ and $2.0 \mathrm{~mA}$ ) were investigated in separate sessions. Using transcranial magnetic stimulation (TMS), 25 motor-evoked potentials (MEPs) were recorded before, and 10 time points up to $2 \mathrm{~h}$ following $15 \mathrm{~min}$ of tDCS. Repeated-measures ANOVAs indicated a main effect of intensity for both anodal and cathodal tDCS. With anodal tDCS, all active intensities resulted in equivalent facilitatory effects relative to sham while for cathodal tDCS, only $1.0 \mathrm{~mA}$ resulted in sustained excitability diminution. An additional experiment conducted to assess intra-individual variability revealed generally good reliability of $1.0 \mathrm{~mA}$ anodal tDCS (ICC $(2,1)=0.74$ over the first $30 \mathrm{~min}$ ). A post hoc analysis to discern sources of inter-individual variability confirmed a previous finding in which individual 
TMS SI $I_{1 \mathrm{mV}}$ (stimulus intensity for $1 \mathrm{mV}$ MEP amplitude) sensitivity correlated negatively with $1.0 \mathrm{~mA}$ anodal tDCS effects on excitability. Our study thus provides further insights on the extent of non-linear intensity-dependent neuroplastic after-effects of anodal and cathodal tDCS.

(Received 2 May 2016; accepted after revision 4 October 2016; first published online 10 October 2016)

Corresponding author M. A. Nitsche: Department of Psychology and Neurosciences, Leibniz Research Centre for

Working Environment and Human Factors, Ardeystr. 67, 44139 Dortmund, Germany. Email: nitsche@ifado.de

Abbreviations ADM, abductor digiti muscle; M1, primary motor cortex; MEP, motor-evoked potential; $\mathrm{SI}_{1 \mathrm{mV}}$, stimulus intensity for $1 \mathrm{mV}$ MEP amplitude; $\mathrm{TDCS}$, transcranial direct current stimulation; TMS, transcranial magnetic stimulation.

\section{Introduction}

Development of non-invasive methods of modulating neuroplasticity is a major ambition in clinical and cognitive neuroscience. In the last decades, tools based on electric and magnetic stimulation such as paired associative stimulation (PAS), repetitive transcranial magnetic stimulation (rTMS), theta-burst stimulation (TBS), and transcranial direct current stimulation (tDCS) have shown potential to induce neuroplastic changes in the human motor cortex (Pascual-Leone et al. 1994; Jennum et al. 1995; Nitsche \& Paulus, 2000; Stefan et al. 2000). The latter has especially surged in recent years, owing to the non-invasive and painless method of delivering weak direct currents to induce cortical plasticity via subthreshold neuronal membrane polarization (Nitsche \& Paulus, 2001). Neuroplastic after-effects of tDCS, as reported in most cases when in a relaxed state, are polarity dependent: anodal stimulation results in facilitation of motor cortical excitability whereas cathodal tDCS diminishes it (Nitsche \& Paulus, 2000, 2001; Nitsche et al. 2003b). Primary studies on tDCS focused on methodological and physiological aspects, uncovering the role of the $\mathrm{N}$-methyl-D-aspartate (NMDA) receptor and calcium channel dependency in achieving effects on motor cortical plasticity (Liebetanz et al. 2002; Nitsche et al. 2003a, 2008; Stagg \& Nitsche, 2011). Recent studies have implemented tDCS in a variety of research and clinical settings, and have shown its ability to modulate cognitive functions and improve a range of neurological and psychiatric impairments (Kuo \& Nitsche, 2012; Flöel, 2014; Kuo et al. 2014; Shin et al. 2015; Woods et al. 2015).

A parallel objective has been optimizing tDCS for enhanced and prolonged effects. For anodal tDCS, our earliest studies indicated that stronger and longer stimulation tend to induce greater effects (Nitsche \& Paulus, 2000, 2001). Clinical studies have since used stimulation durations up to $20-30 \mathrm{~min}$ with current intensities up to $2.0 \mathrm{~mA}$ and have achieved positive results (Boggio et al. 2009; Brunoni et al. 2013; Shekhawat et al. 2013). In the healthy population, we have shown that increasing stimulation duration or intensity have not produced concomitant physiological effects in each case. When anodal tDCS was prolonged to $26 \mathrm{~min}$ after-effects were converted into excitability diminution (Monte-Silva et al. 2013). However, when two $13 \mathrm{~min}$ blocks of anodal tDCS were spaced by $20 \mathrm{~min}$, after-effects were present for up to $24 \mathrm{~h}$, suggesting involvement of late-phase long-term potentiation (LTP) plasticity (Monte-Silva et al. 2013). When 20 min cathodal tDCS was increased from 1.0 to $2.0 \mathrm{~mA}$, after-effects were reversed (Batsikadze et al. 2013), and in other studies, anodal tDCS at varying current intensities also resulted in a non-linear pattern of after-effects (Bastani \& Jaberzadeh, 2013; Kidgell et al. 2013). Importantly, however, the entire range of DC intensities has not been systematically investigated, particularly in a within-subject repeated measure design, which could uncover dosage-dependent insights on mechanistic properties while also reliably accounting for individual effects. The need for these studies is further underscored by reported findings of inter-individual variability (López-Alonso et al. 2014; Wiethoff et al. 2014; Chew et al. 2015; Strube et al. 2015), possibly due in part to relevant individual covariates such as demographics, genetics, cortical anatomy, attention and/or sensitivity to stimulation (Kuo et al. 2006; Ridding \& Ziemann, 2010; Labruna et al. 2015; Opitz et al. 2015).

In the following study, we systematically investigated the effects of anodal and cathodal tDCS at five current intensities (sham, 0.5, 1.0, 1.5 and $2.0 \mathrm{~mA}$ ) on motor-cortical plasticity, measured via changes in TMS-induced motor evoked potentials (MEP). We hypothesized that current intensity has a non-linear modulatory effect on neuroplasticity, as has been observed with other stimulation parameters (Monte-Silva et al. 2010, 2013; Batsikadze et al. 2013). Moreover, we investigated a possible cause of the inter-individual variability in response to different tDCS intensities, correlating the efficacy of tDCS with the baseline sensitivity to TMS, which was recently found to be a significant covariate (Labruna et al. 2015). Our study thus aims to advance the methodological parameters and considerations of $\mathrm{tDCS}$, which are important for achieving prolonged physiological effects. 


\section{Methods}

\section{Ethical approval}

The study conformed to the Declaration of Helsinki and was approved by the Medical Ethics Committee of the University of Göttingen. Each subject provided written informed consent before beginning the study, and was compensated for participation.

\section{Subjects}

Thirty-eight healthy, non-smoking participants ( 17 males, 21 females, mean age $25.80 \pm 4.41$ years) were recruited for the study. All subjects were right-handed as assessed by the Edinburgh handedness inventory (Oldfield, 1971). Prio to taking part, participants underwent a medical screening to verify no history of neurological disease, medication metal implants, and pregnancy. Each subject first took part in a preliminary TMS session to become acquainted with experiencing stimulation and understanding the study protocol. Subjects were instructed not to consume caffeine, alcohol, or engage in strenuous physical activities $24 \mathrm{~h}$ prior to each session to ensure a stable leve of motor-cortical excitability. Subjects were randomly allotted to receive either anodal or cathodal stimulation only over the course of the five pseudo-randomized (uniformly distributed) experimental sessions of different intensities (sham, 0.5, 1.0, 1.5, and $2.0 \mathrm{~mA}$ ), which were separated by at least 7 days to avoid carry-over effects. Subjects were blinded to their group (polarity) and session (intensity) and 33 out of the 38 subjects were naïve to tDCS.

\section{Stimulation of the motor cortex}

Following baseline measurements of cortical excitability, participants were given $15 \mathrm{~min}$ of direct current stimulation through a pair of saline soaked sponges placed on the scalp and delivered through a constant-current battery powered stimulator (neuroConn, Ilmenau, Germany). A $15 \mathrm{~min}$ stimulation is in the range of stimulation protocols producing polarity-specific long-term effects with $1 \mathrm{~mA}$ stimulation, without inducing late phase or converted effects, which might limit the observability of an altered impact of stimulation with larger intensity (Monte-Silva et al. 2010, 2013). A $35 \mathrm{~cm}^{2}$ target electrode was fixed over the motor-cortical position of the right abductor digiti muscle (ADM) as identified by TMS (electrode rotated $45 \mathrm{deg}$ towards the midline, with the cable leaving from the middle of the right edge). Another larger $100 \mathrm{~cm}^{2}$ electrode was placed contralaterally over the right orbit in order to reduce the current density and also unwanted effects under this region (Nitsche et al. 2007). For all subjects, the distance on the scalp between the edges of the electrodes was at least $6 \mathrm{~cm}$. To further reduce any discomfort due to the higher intensities of stimulation, a topical anaesthetic cream (EMLA, $2.5 \%$ lidocaine $+2.5 \%$ prilocaine) was pre-applied to the scalp under the electrodes, which has been shown to effectively reduce perception of the stimulation and ensure adequate blinding (McFadden et al. 2011; Guleyupoglu et al. 2014). Based on the randomized group and session condition, anodal or cathodal tDCS at an intensity of $0.5,1.0,1.5$ or $2.0 \mathrm{~mA}$ was delivered for $15 \mathrm{~min}$ with a $10 \mathrm{~s}$ ramp at the beginning and end of stimulation. For the sham condition, stimulation was delivered at $1 \mathrm{~mA}$ for $30 \mathrm{~s}$, with a $20 \mathrm{~s}$ ramp. Using this procedure, subjects are not able to distinguish between real and sham tDCS (Gandiga et al. 2006; Ambrus et al. 2012). After $15 \mathrm{~min}$, electrodes were removed and corticospinal excitability was monitored with TMS.

\section{EMG monitoring of motor cortical excitability from TMS}

Single pulse monophasic TMS at $0.25 \mathrm{~Hz}$ was delivered by a Magstim 200 magnetic stimulator (Magstim, Whiteland, UK) through a figure- 8 magnetic coil held $45^{\circ}$ to the midline, with current flowing posterior-anterior (diameter of one winding $=70 \mathrm{~mm}$, peak magnetic field $=2.2 \mathrm{~T}$ ). Electromyography was recorded from $\mathrm{Ag}-\mathrm{AgCl}$ electrodes attached to the ADM of the right hand in a belly-tendon montage. Signals were sampled at $5 \mathrm{kHz}$ (CED 1401, Cambridge, UK), amplified and bandpass filtered at $2 \mathrm{~Hz}-2 \mathrm{kHz}$ (Digitimer, Welwyn Garden City, UK). All EMG measures were recorded with Signal software (CED) and analysed with in-house scripts written in Python v2.7 (stimfit library, version 0.11.5; http://www. stimfit.org/).

\section{Experimental procedure}

Experiment 1. Participants were seated comfortably in a reclined chair, with a pillow resting under the right arm. At the beginning of each session, baseline cortical excitability was measured by first inducing MEPs over the left M1 to identify the region which produced the largest MEP of the target muscle. The region was then marked and subsequent pulses for the duration of the session were delivered from this optimal position. The stimulator's intensity was adjusted to reach a peak-to-peak MEP amplitude of $1 \mathrm{mV}\left(\mathrm{SI}_{1 \mathrm{mV}}\right)$, which was then used for the remaining measurements. Following a baseline measurement of $25 \mathrm{MEPs}, 15 \mathrm{~min}$ of anodal or cathodal stimulation was delivered as previously described. After removal of tDCS electrodes, MEP measurements were taken immediately again in epochs of every $5 \mathrm{~min}$ up to $30 \mathrm{~min}$ after the stimulation, and then every $30 \mathrm{~min}$ up to $2 \mathrm{~h}$ after stimulation (11 total epochs) (Fig. 1). 
Experiment 2. An additional control experiment was conducted to assess intra-individual variability of $1.0 \mathrm{~mA}$ anodal tDCS, using the same procedures. Seven participants from the original cohort participated in two additional sessions, in which $1.0 \mathrm{~mA}$ tDCS was delivered for $15 \mathrm{~min}$, and motor-cortical excitability was monitored for up to $2 \mathrm{~h}$ following the end of stimulation. Data acquisition and subsequent analysis was repeated in the exact same manner as Experiment 1.

\section{Data analysis and statistics}

Baseline measures. To determine if individual baseline measures differed between session, $\mathrm{SI}_{1 \mathrm{mV}}$ and Baseline MEP were entered as dependent variables in a repeatedmeasures ANOVA with session as a within-subject factor.

Experiment 1. The peak-to-peak amplitude of the 25 MEPs for each time epoch was calculated and averaged together. To obtain a time series of a subject's change in excitability over the session, the mean MEP amplitude for each measurement time epoch was normalized to the session's baseline (a quotient of the mean from the baseline mean) resulting in values representing either increased $(>1.0)$ or decreased $(<1.0)$ excitability. The normalized MEPs from each epoch were then entered as dependent variables into a two-way repeated-measures ANOVA, with the independent variables of intensity (5 levels) and time (10 levels) as within-subject factors. Mauchly's test of sphericity was conducted, and Greenhouse-Geisser correction was applied when necessary. Statistical analysis was repeated in the same manner for cathodal stimulation. In the case of significant effects, follow-up post hoc Student's paired $\mathrm{t}$ tests (two-tailed) were conducted to examine if an active intensity resulted in a significant difference relative to sham or baseline.

Experiment 2. For Experiment 2, analysis proceeded in the same manner as Experiment 1. The baselinenormalized time series for each individual across the three repeated sessions was then grand-averaged over two time bins: the first $30 \mathrm{~min}$, relating to the early plasticity changes, and over $60-120 \mathrm{~min}$, relating to the later excitability changes. Finally, intra-individual variability was calculated for these time bins using the intra-class correlation coefficient, $\operatorname{ICC}(2,1)$, to assess the absolute agreement of individual responses (Shrout \& Fleiss, 1979).

\section{Inter-individual variability analysis}

As a post hoc analysis, we investigated sources of inter-individual variability in our dataset, which has recently been reported as a relevant issue from similar studies (López-Alonso et al. 2014; Wiethoff et al. 2014;

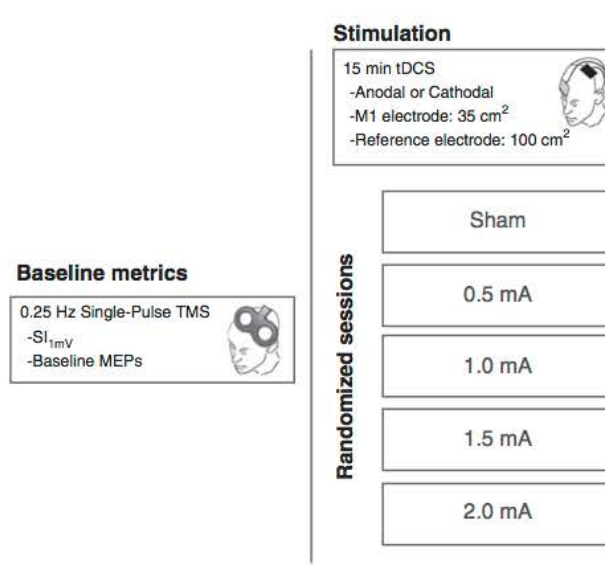

Post-stimulation monitoring

$0.25 \mathrm{~Hz}$ Single-Pulse TMS

25 MEPS

Every $5 \mathrm{~min}$ up to $30 \mathrm{~min}$

$60 \mathrm{~min}$

$90 \mathrm{~min}$

$120 \mathrm{~min}$

Figure 1. Course of study

Participants were randomly divided into two groups for tDCS polarity (Anodal: $n=20$; Cathodal: $n=18$ ). Each participant took part in five randomized sessions during which either sham, $0.5,1.0,1.5$ or 2.0 mA stimulation with the respective polarity was applied. Prior to receiving stimulation, baseline MEP amplitude and S $1_{1 \mathrm{mv}}$ was measured over the determined motor cortical 'hotspot' which produced the largest MEP from the right ADM muscle. Next, DC stimulation for $15 \mathrm{~min}$ was delivered, and MEP measurements were taken again from the hotspot immediately after stimulation, as well as every $5 \mathrm{~min}$ up to $30 \mathrm{~min}$, and then every $30 \mathrm{~min}$ up to $2 \mathrm{~h}$ after stimulation. 


\begin{tabular}{|c|c|c|c|c|c|c|}
\hline \multirow[b]{2}{*}{ Experiment } & \multirow{2}{*}{$\begin{array}{l}\text { Experimental } \\
\text { session }\end{array}$} & \multicolumn{3}{|c|}{ Subjects } & \multirow[b]{2}{*}{$\mathrm{Sl}_{1 \mathrm{mV}}(\%)$} & \multirow[b]{2}{*}{ Baseline MEP ( $\mathrm{mV}$ ) } \\
\hline & & $n$ & $\operatorname{Sex}(M / F)$ & Age (years) & & \\
\hline \multirow[t]{5}{*}{ Anodal stimulation } & Sham & 20 & $10 / 10$ & $25.7 \pm 4.66$ & $46.9 \pm 10.01$ & $1.03 \pm 0.22$ \\
\hline & $0.5 \mathrm{~mA}$ & 20 & $10 / 10$ & $25.7 \pm 4.66$ & $48.28 \pm 9.67$ & $0.90 \pm 0.23$ \\
\hline & $1.0 \mathrm{~mA}$ & 20 & $10 / 10$ & $25.7 \pm 4.66$ & $46.76 \pm 9.64$ & $0.98 \pm 0.22$ \\
\hline & $1.5 \mathrm{~mA}$ & 20 & $10 / 10$ & $25.7 \pm 4.66$ & $47.67 \pm 10.83$ & $0.94 \pm 0.17$ \\
\hline & $2.0 \mathrm{~mA}$ & 20 & $10 / 10$ & $25.7 \pm 4.66$ & $48.19 \pm 11.30$ & $0.95 \pm 0.27$ \\
\hline \multirow[t]{5}{*}{ Cathodal stimulation } & Sham & 18 & $7 / 11$ & $26.2 \pm 4.72$ & $44.61 \pm 8.75$ & $0.99 \pm 0.14$ \\
\hline & $0.5 \mathrm{~mA}$ & 18 & $7 / 11$ & $26.2 \pm 4.72$ & $44.16 \pm 8.77$ & $0.99 \pm 0.13$ \\
\hline & $1.0 \mathrm{~mA}$ & 18 & $7 / 11$ & $26.2 \pm 4.72$ & $45.01 \pm 9.01$ & $0.91 \pm 0.16$ \\
\hline & $1.5 \mathrm{~mA}$ & 18 & $7 / 11$ & $26.2 \pm 4.72$ & $44.89 \pm 9.49$ & $1.00 \pm 0.24$ \\
\hline & $2.0 \mathrm{~mA}$ & 18 & $7 / 11$ & $26.2 \pm 4.72$ & $44.05 \pm 8.96$ & $0.98 \pm 0.20$ \\
\hline
\end{tabular}

The number of subjects for each experimental condition is listed, along with the gender distribution, the mean age, and baseline TMS metrics $( \pm S D)$. $\left.S\right|_{1 m v}$ refers to the stimulus intensity required to produce an average motor evoked potential (MEP) of $1 \mathrm{mV}$. Baseline MEP refers to the average amplitude of the 25 baseline recordings. No factor differed significantly between session and experimental group.

Table 2. Main effects analysis from ANOVAs

\begin{tabular}{|c|c|c|c|c|c|}
\hline Experiment & Measurement & Factor & d.f. & $F$ value & $P$ value \\
\hline \multirow[t]{5}{*}{ Anodal stimulation } & Baseline MEP & Session & 4 & 1.608 & 0.181 \\
\hline & $\mathrm{SI}_{1 \mathrm{mV}}$ & Session & 4 & 1.508 & 0.208 \\
\hline & MEP & Intensity & 4 & 3.25 & $0.016^{*}$ \\
\hline & MEP & Time & 4.37 & 5.603 & $<0.001^{*}$ \\
\hline & MEP & Intensity $\times$ time & 9.74 & 1.384 & 0.193 \\
\hline \multirow[t]{5}{*}{ Cathodal stimulation } & Baseline MEP & Session & 4 & 0.826 & 0.513 \\
\hline & $\mathrm{SI}_{1 \mathrm{mV}}$ & Session & 4 & 0.683 & 0.606 \\
\hline & MEP & Intensity & 4 & 3.135 & $0.020^{*}$ \\
\hline & MEP & Time & 3.252 & 1.790 & 0.156 \\
\hline & MEP & Intensity $\times$ time & 7.532 & 0.792 & 0.603 \\
\hline
\end{tabular}

First, a one-way ANOVA was calculated for inter-session differences of the average baseline motor evoked potential (MEP) amplitude as well as the TMS stimulus intensity for $1 \mathrm{mV}$ amplitude $(S \mid \mathrm{mV})$. A two-way repeated-measures ANOVA was calculated for main effects of stimulation intensity and post-stimulation time on MEP size. "Significant results (where $P<0.05$ ). Baseline MEP and SI1mV did not significantly differ across session for either experimental group. There was a main effect of intensity for both anodal and cathodal stimulation, and a main effect of time for anodal stimulation.

Strube et al. 2015). We first investigated subject-specific baseline sensitivity to TMS (defined as percentage of maximum stimulator output (\%MSO) required for the $\mathrm{SI}_{1 \mathrm{mV}} \mathrm{MEP}$ amplitude), which was recently identified as a contributing covariate that may influence subject-level response to tDCS (Labruna et al. 2015). For direct comparison, we replicated the statistical methods by first median-splitting our subject pool into two groups by average $\mathrm{SI}_{1 \mathrm{mV}}\left(\mathrm{SI}_{1 \mathrm{mV}}\right.$ Low and High) and conducting between-group comparisons of the grand averaged first $30 \mathrm{~min}\left(\mathrm{MEP}_{\text {Early epoch }}\right)$ and final $60-120 \mathrm{~min}$ $\left(\mathrm{MEP}_{\overline{\text { Late epoch }}}\right)$. Averaged data of each subject were entered into separate ANOVAs (five intensities), with intensity as a within-subject factor, $\mathrm{SI}_{1 \mathrm{mV}}$ group (High and Low) as a between-subject factor, and MEP Early epoch $_{\text {and }}$

@ 2016 The Authors. The Journal of Physiology @ 2016 The Physiological Society
$\mathrm{MEP}_{\text {Late epoch }}$ as dependent variables. For significant effects and interactions, follow-up tests were conducted using Student's unpaired $t$ test (two-tailed). In addition, we also calculated correlation coefficients for each intensity, using $\mathrm{SI}_{1 \mathrm{mV}}$ as one variable and the grand-average MEP as a second variable. Finally, factors of gender and age were also investigated, as these have also been previously identified in relevant brain stimulation studies (Kuo et al. 2006; Bashir et al. 2014; Wiethoff et al. 2014). These factors were analysed with the same steps, using separate ANOVAs to model the covariate interaction. Note that for the case of $1.0 \mathrm{~mA}$ anodal tDCS, only the first session data for each individual was used, in order to maintain homogeneity of the randomized sampling. 
Statistical analysis was performed with SPSS (IBM Corp. Version 22.0). Inference testing for post hoc $t$ tests was set to a $P$ value of 0.05 (not corrected for multiple comparisons). Pairwise effect sizes are presented as Cohen's $d$. Note that effect sizes were computed based on the difference from baseline, in order to represent the relative change in post-stimulation excitability.

\section{Results}

All subjects tolerated all intensities of stimulation, including the highest intensity of $2.0 \mathrm{~mA}$. Some subjects reported an itching/tingling sensation during the beginning of the stimulation, which eventually faded away after a few minutes. In a few subjects, we observed reddening of the skin under the scalp electrode, which did not persist for longer than $60 \mathrm{~min}$. Reliability of the blinding was not quantitatively assessed in the present study, although in a previous pilot study from our group, most participants were unable to distinguish between the current intensities investigated here (Ambrus et al. 2010), and local anaesthetic cream relevantly reduces tDCS-induced sensory perceptions (McFadden et al. 2011). No other adverse effects were reported.

Descriptive statistics of demographics as well as baseline measures of motor-cortical excitability are summarized in Table 1. An overall ANOVA indicated that baseline MEP and $\mathrm{SI}_{1 \mathrm{mV}}$ did not significantly differ across sessions for either group (all values of $P>0.05$; Table 2).
Intensity-dependent effects of cortical excitability

Anodal stimulation. The overall ANOVA indicated an effect of intensity $(F=3.25$, d.f. $=4, P=0.016)$, and time $(F=5.603$, d.f. $=4.37, P<0.001)$, but no intensity $\times$ time interaction $(F=1.384$, d.f. $=9.74$ $P=0.193$; Table 2). Post hoc comparisons to sham revealed that all active intensities of anodal $\mathrm{tDCS}$ resulted in a significant post-stimulation increase in cortical excitability across most of the early (0-30 $\mathrm{min})$ time epochs, but between active intensities there were no significant differences (Fig. $2 A$ ). Sham stimulation did not result in a change of cortical excitability. A comparison of the effect sizes between the active intensities and sham across the two time bins (0-30 $\mathrm{min}$ and 60-120 $\mathrm{min}$ ) revealed a generally non-linear pattern whereby the lowest intensity of $0.5 \mathrm{~mA}$ and the highest intensity of $2.0 \mathrm{~mA}$ led to marginally greater effects during both early and late time points compared to $1.0 \mathrm{~mA}$ and $1.5 \mathrm{~mA}$ $(d=0.74$ and 0.80 for $0.5 \mathrm{~mA}$ and $2.0 \mathrm{~mA}$, respectively; Fig. 2B).

Cathodal stimulation. ANOVA results indicated an effect of intensity $(F=3.315$, d.f. $=4, P=0.020)$, but no effect of time $(F=1.790$, d.f. $=3.252, P=0.156)$ or intensity $\times$ time interaction $(F=0.792$, d.f. $=7.532$, $P=0.603$; Table 2). Interestingly, we did not observe a linear trend of cortical excitability diminution; 1.5 and $2.0 \mathrm{~mA}$ intensities tended to return excitability to

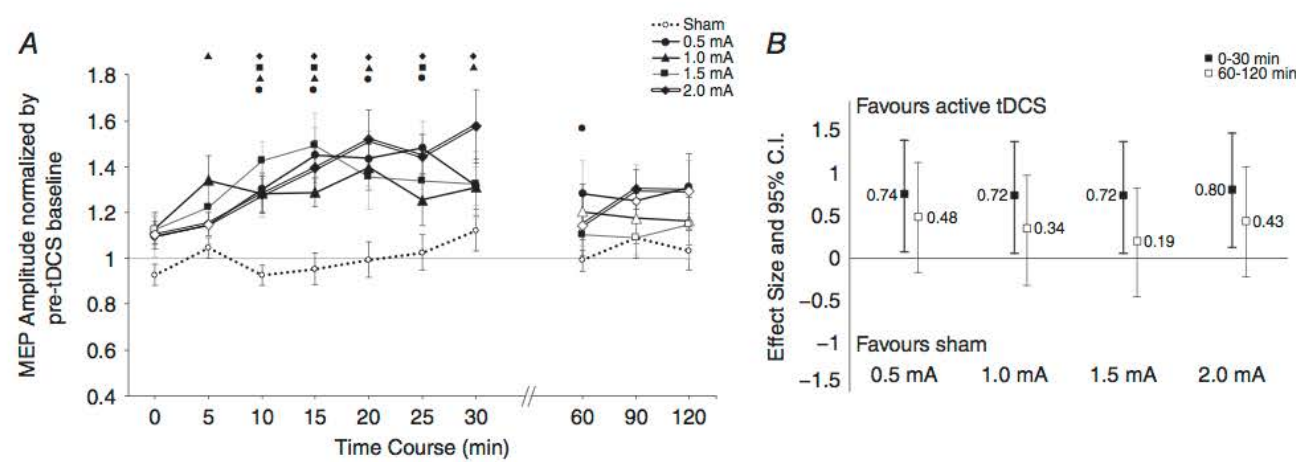

Figure 2. Intensity-dependent effects in motor-cortical excitability following anodal tDCS

$A$, after-effects of cortical excitability following $15 \mathrm{~min}$ of anodal stimulation at intensities ranging from sham to $2.0 \mathrm{~mA}$ on the mean MEP amplitude. Error bars represent standard error. Filled symbols indicate a significant difference in cortical excitability against the respective baseline (Student's paired $t$ test, two-tailed, $P<0.05$ ). Floating symbols $(0.5,1.0 ; 1.5,2.0 \mathrm{~mA}$ ) indicate a significant difference between the active intensity and sham stimulation (paired $t$ test, two-tailed, $P<0.05$ ). Anodal stimulation over in significant increases of excitability lasting up to $30 \mathrm{~min}$. Sham stimulation did not induce any significant change in cortical excitability. $B$, effect sizes and $95 \%$ confidence intervals of active tDCS intensities versus sham. MEP amplitudes were averaged into two time bins of early $(0-30 \mathrm{~min})$ and late $(60-120 \mathrm{~min})$ excitability changes, followed by calculation of Cohen's effect size $d$. Error bars represent $95 \%$ confidence intervals based on the pooled variance. Differences between active intensities were generally not discernable in the first $30 \mathrm{~min}$; however, 0.5 and $2.0 \mathrm{~mA}$ resulted in slightly larger effects, especially in the time window $60-120 \mathrm{~min}$. 
baseline values after just a few minutes following stimulation (Fig. 3A). Sham stimulation resulted in no effect. Post hoc tests indicated only a significant effect of $1.0 \mathrm{~mA}$ stimulation when compared to both baseline values as well as against sham. The magnitude of the effects of $1.0 \mathrm{~mA}$ cathodal tDCS relative to sham was greater in the later epoch (60-120 $\mathrm{min})$ compared to earlier recordings (all values of $P<0.05 ; d=0.61$ and 0.69 for pooled early and late time bins, respectively). Overall, only the lower intensities of $0.5 \mathrm{~mA}$ and $1.0 \mathrm{~mA}$ appeared to account for any sizable variance in MEP amplitude in the whole group analysis (Fig. 3B).

\section{Reliability and intra-individual variability}

To assess whether variability among the MEPs collected within each time point changed with tDCS intervention, we repeated the previous analysis, this time operationalizing the standard error of the mean (SEM) over the 25 MEPs at each of the 11 measurements in the session. For both anodal and cathodal tDCS, a repeated-measures ANOVA indicated no significant effect of intensity (anodal: $F=0.372$, d.f. $=2.293, P=0.720$; cathodal: $F=1.364$, d.f. $=4, P=0.256$ ), time (anodal: $F=2.009$, d.f. $=10, P=0.154$; cathodal: $F=2.839$, d.f. $=10, P=0.076)$, or intensity $\times$ time interaction (anodal: $F=1.166$, d.f. $=10.597, P=0.314$; cathodal:
$F=0.797$, d.f. $=40, P=0.811)$. These results indicate that within each measurement time point, variance in the collected MEPs did not significantly change as a result of tDCS or time. To assess the reliability and intra-individual variability of the post-stimulation cortical excitability modulation, we repeated $1.0 \mathrm{~mA}$ anodal tDCS in two additional sessions within a subgroup of 7 subjects. For the early epoch (first $30 \mathrm{~min}$ ), the grand-averaged post-tDCS MEP response between the three sessions resulted in $\operatorname{ICC}(2,1)=0.738$, suggesting good agreement (Cicchetti, 1994). Reliability in the average MEP amplitude modulation during the late epoch $(60-120 \mathrm{~min})$ was also considered good, $\operatorname{ICC}(2,1)=0.642$. ICC values were also obtained for each individual time point, for means of comparison to Lopez-Alonso et al. (2015), and in general accordance, the greatest reproducibility in cortical excitability modulation was observed during the period 15-25 min following tDCS (Fig. 4).

\section{Dependency of cortical excitability after-effects} on baseline sensitivity to TMS

Anodal stimulation. We conducted a post hoc subgroup analysis to determine if a participant's baseline sensitivity to TMS (measured as $\mathrm{SI}_{1 \mathrm{mV}}$ ) may explain the inter-individual variability in the post-stimulation response to anodal and cathodal tDCS. We first calculated,

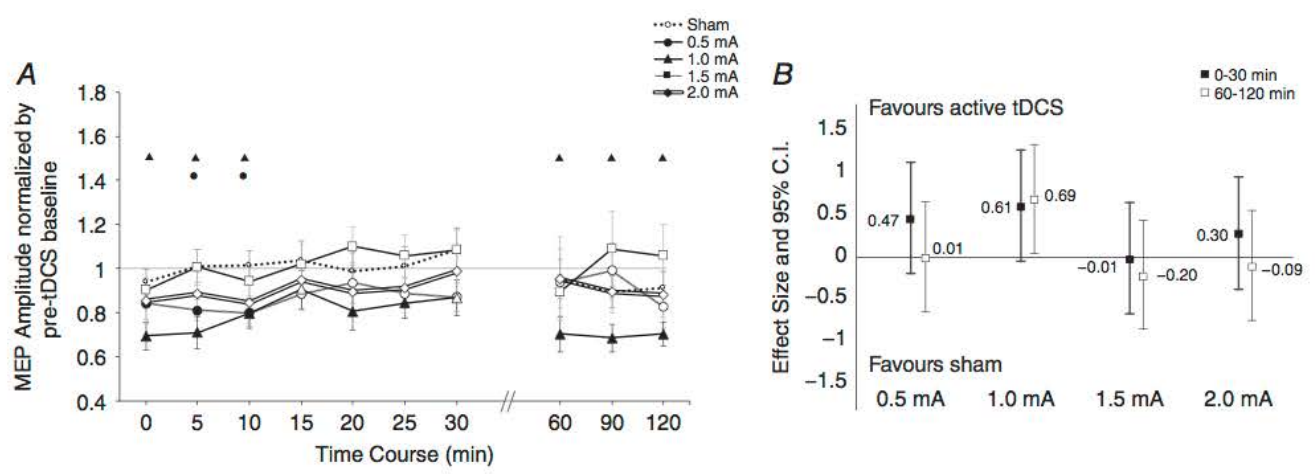

Figure 3. Intensity-dependent effects in motor-cortical excitability following cathodal tDC $A$, after-effects of cortical excitability following $15 \mathrm{~min}$ of cathodal stimulation at intensities ranging from sham A, after-effects of cortical excitability following $15 \mathrm{~min}$ of cathodal stimulation at intensities ranging from sham difference in cortical excitability against the respective baseline (paired $t$ test, two-tailed, $P<0.05$ ). Floating symbols $(\bullet, 0.5 ; \Delta, 1.0 ; \mathbf{\square}, 1.5 ;, 2.0 \mathrm{~mA})$ indicate a significant difference between the active intensity and sham stimulation (paired $t$ test, two-tailed, $P<0.05$ ). Only $0.5 \mathrm{~mA}$ and $1.0 \mathrm{~mA}$ cathodal stimulation resulted in significant differences from baseline, and only $1.0 \mathrm{~mA}$ was significantly different from sham during the early time bins. Higher intensities such as 1.5 and $2.0 \mathrm{~mA}$ (the latter is highlighted with double lines) tended to return to baseline values after about $10 \mathrm{~min}$. Sham stimulation did not induce any significant change in cortical excitability. $B$, effect sizes and $95 \%$ confidence intervals of active tDCS intensities versus sham. MEP amplitudes were averaged into two time bins of early $(0-30 \mathrm{~min})$ and late $(60-120 \mathrm{~min})$ excitability changes, followed by calculation of Cohen's effect size d. Error bars represent $95 \%$ confidence intervals based on the pooled variance. Note that larger effects correspond to greater reduction of excitability from baseline (see Methods). Greatest differences are again seen with lower intensities of 0.5 and $1.0 \mathrm{~mA}$ whereas higher intensities did not result in marked changes. 
for each subject, the average $\mathrm{SI}_{1 \mathrm{mV}}$ over the five sessions (note that $\mathrm{SI}_{1 \mathrm{mV}}$ did not statistically differ between sessions; $\mathrm{SD}=1.99, P>0.05$ ) and then split the sample by the median (anodal: $48, n=10$ per group; cathodal: $46, n=9$ per group). For the early epoch (0-30 $\mathrm{min})$, an ANOVA revealed main effects for the factor intensity $(F=3.971$, d.f. $=4, P=0.006)$ as well as the intensity $\times$ group interaction $(F=2.820$, d.f. $=4, P=0.031$; Table 4). Comparisons between groups indicated a significant between-group difference with $1.0 \mathrm{~mA}$ anodal tDCS $(P=0.038, d=0.91$; Fig. $5 A$ and $B)$. For the late epoch $(60-120 \mathrm{~min})$, we did not observe a significant effect for either a factor of intensity or an intensity $\times \mathrm{SI}_{1 \mathrm{mV}}$ group interaction (Table 3 ). We also investigated the entire data set in a continuous manner, and calculated a correlation coefficient between the $\mathrm{SI}_{1 \mathrm{mV}}$ and the average MEP of the early epoch. Similarly, we observed that with $0.5 \mathrm{~mA}$, the correlation between an individual's $\mathrm{SI}_{1 \mathrm{mV}}$ and his/her grand-average response to stimulation tended to be negative (Pearson's $r=-0.182$, $P=0.442$ ), and at $1.0 \mathrm{~mA}$, the correlation was significantly negative $(r=-0.474, P=0.035)$. At $1.5 \mathrm{~mA}$ and $2.0 \mathrm{~mA}$, the correlation tended to be positive, however not statistically significant $(r=0.405, P=0.076$ and $r=0.081$, $P=0.773$, respectively; Fig. 7$)$.

Cathodal stimulation. No significant effects were detected for the late epoch. For the early epoch, the ANOVA indicated a significant factor of intensity $(F=2.765$, d.f. $=4, P=0.035)$, but no significant intensity $\times \mathrm{SI}_{1 \mathrm{mV}}$ group interaction $(F=0.732$, d.f. $=4, P=0.573)$. When comparing individual intensities, we did not detect any clear pattern of an intensity-dependent relationship (Fig. 5C). Similarly, when the data were analysed as a continuum, Pearson's correlation coefficient was not statistically significant at any intensity of active stimulation (Table 4). A comparison of the relative effect sizes indicated that the largest between-group differences were seen with

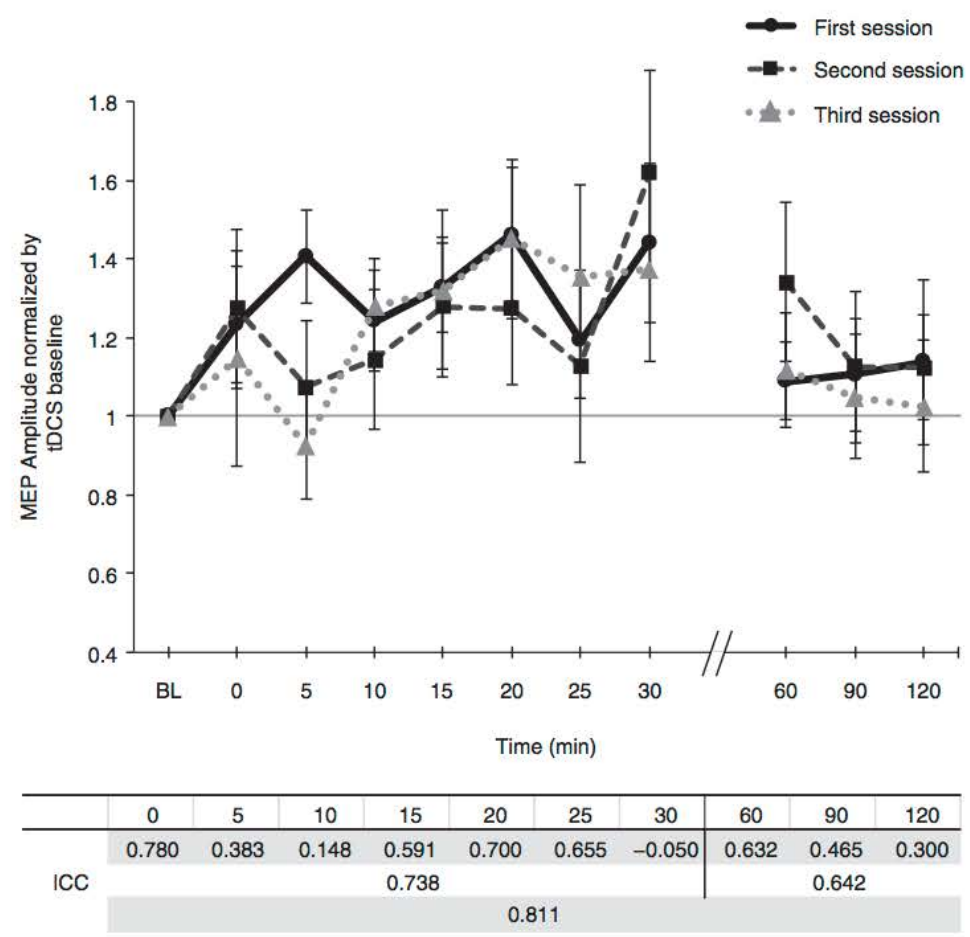

Figure 4. Intra-individual response to $1.0 \mathrm{~mA}$ anodal tDCS

Time courses of cortical excitability, measured as MEP amplitudes normalized to baseline, in a subgroup of 7 participants across three separate sessions are shown. Error bars represent standard error. In the table below, the intra-class correlation coefficient $(I C C(2,1))$ was used to assess the strength of the reliability and reproducibility of $1.0 \mathrm{~mA}$ anodal tDCS, at each time point (first row), as well as over first $30 \mathrm{~min}$ and final $60 \mathrm{~min}$ (second and third row, respectively). 
$0.5 \mathrm{~mA}$ (early time bins favouring the Low $\mathrm{SI}_{1 \mathrm{mV}}$ group; $d=0.32$ ) and $2.0 \mathrm{~mA}$ (both early and late time bins favouring the High $\mathrm{SI}_{1 \mathrm{mV}}$ group; $d=-0.68$ and -0.57 , respectively; Fig. 5D).

\section{No dependency of cortical excitability on age or gender}

We also investigated if variance of the post-stimulation after-effects may be explained by differences in gender or age by including these factors as covariates in separate ANOVAs. No interaction was observed for either age or gender (Table 3 ).

\section{Discussion}

In the present study, we systematically evaluated anodal and cathodal current intensities and observed generally non-linear intensity-dependent effects on motor cortical

Anodal tDCS
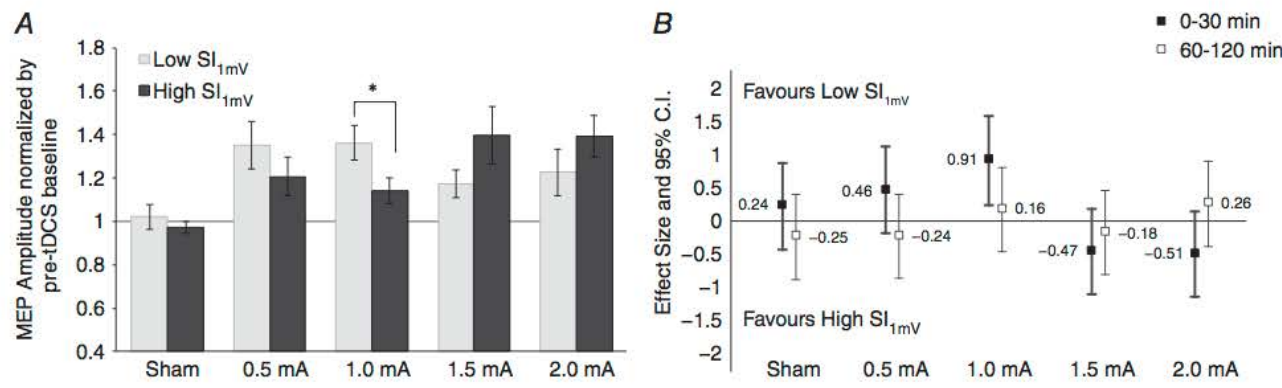

Cathodal tDCS

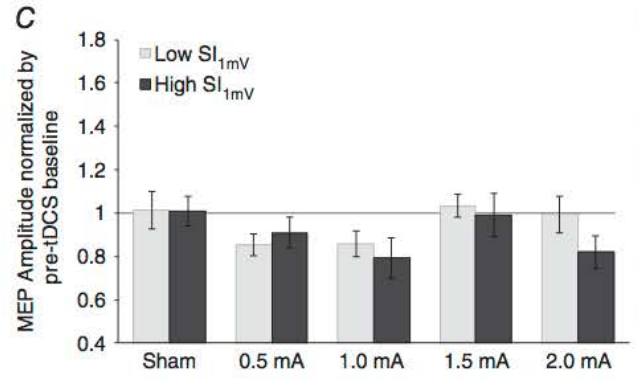

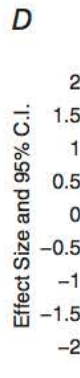

Figure 5. Differences in anodal and cathodal tDCS-induced excitability between subjects with low and high sensitivity to TMS

The sample was median-split (anodal: $48 \%$; cathodal: $46 \%$ ) with 'Low Sl ${ }_{1 \mathrm{mv}}$ ' consisting of participants who required less than the median value to achieve an MEP amplitude of $1 \mathrm{mV}$ and 'High SI $1 \mathrm{mv}$ ' consisting of the rest. Pairwise comparisons (panels $A$ and $C$ ) between subgroups of the pooled average from the first $30 \mathrm{~min}$ were conducted using Student's unpaired, two-tailed $t$ test. Error bars represent the standard error. *Significant differences between the groups $(P<0.05$ ). Effect size comparisons (panels $B$ and $D$ ) were conducted by calculating Cohen's $d$. Error bars represent $95 \%$ confidence intervals of the pooled variance. A, subjects with Low SI 1 mv showed significantly greater increases in $1.0 \mathrm{~mA}$ anodal tDCS compared to subjects with High Slimv. Note that within the sub-groups, $1.0 \mathrm{~mA}$ was not significantly better than $1.5 \mathrm{~mA}(P=0.081)$ for subjects with Low Sh thv and $1.5 \mathrm{~mA}$ was not significantly better than $1.0 \mathrm{~mA}(P=0.073)$ for the subjects with High Slimv. $B$, intensity effects for the first $30 \mathrm{~min}$ appear to follow a trend-wise pattern, whereby lower intensities favour subjects with Low SI ${ }_{1 \mathrm{mV}}$ while higher intensities favour subjects with High SI ${ }_{1 \mathrm{mV}}$. C, a subgroup comparison of cathodal tDCS does not reveal any significant pairwise difference during the first $30 \mathrm{~min}$. $D$, effect size comparisons (where larger effects equate to greater reduction of cortical excitability) show a marginal intensity-dependent tendency for the lowest $(0.5 \mathrm{~mA})$ and highest (2.0 mA) intensity only, which also follows the same pattern as anodal tDCS. 


\begin{tabular}{|c|c|c|c|c|c|}
\hline Experiment & Measurement & Factor & d.f. & $F$ value & $P$ value \\
\hline \multicolumn{6}{|l|}{ Sl ${ }_{1 m v}$} \\
\hline \multirow[t]{4}{*}{ Anodal stimulation } & MEP $_{\text {Early epoch }}$ & Intensity & 4 & 3.971 & $0.006^{*}$ \\
\hline & MEP Early epoch & Intensity $\times \mathrm{SI}_{1 \mathrm{mv}}$ & 4 & 2.820 & $0.031^{*}$ \\
\hline & MEP $_{\text {Late epoch }}$ & Intensity & 4 & 1.216 & 0.311 \\
\hline & MEP Late epoch & Intensity $\times \mathrm{SI}_{1 \mathrm{mv}}$ & 4 & 1.453 & 0.226 \\
\hline \multirow[t]{4}{*}{ Cathodal stimulation } & MEP Early epoch & Intensity & 4 & 2.765 & $0.035^{*}$ \\
\hline & $\mathrm{MEP}_{\text {Early epoch }}$ & Intensity $\times \mathrm{SI}_{1 \mathrm{mv}}$ & 4 & 0.732 & 0.573 \\
\hline & MEP $_{\text {Late epoch }}$ & Intensity & 2.581 & 2.318 & 0.098 \\
\hline & MEP $P_{\text {Late epoch }}$ & Intensity $\times \mathrm{SI}_{1 \mathrm{mv}}$ & 2.581 & 1.056 & 0.371 \\
\hline \multicolumn{6}{|l|}{ Age } \\
\hline \multirow[t]{4}{*}{ Anodal stimulation } & $\mathrm{MEP}_{\text {Early epoch }}$ & Intensity & 4 & 3.750 & $0.008^{*}$ \\
\hline & $\mathrm{MEP}_{\text {Early epoch }}$ & Intensity $\times$ age & 4 & 1.659 & 0.169 \\
\hline & MEP $_{\text {Late epoch }}$ & Intensity & 4 & 1.594 & 0.185 \\
\hline & MEP $_{\text {Late epoch }}$ & Intensity $\times$ age & 4 & 1.264 & 0.292 \\
\hline \multirow[t]{4}{*}{ Cathodal stimulation } & $\mathrm{MEP}_{\text {Early epoch }}$ & Intensity & 4 & 0.174 & 0.951 \\
\hline & MEP $_{\text {Early epoch }}$ & Intensity $\times$ age & 4 & 0.151 & 0.962 \\
\hline & MEP Late epoch & Intensity & 2.508 & 1.843 & 0.132 \\
\hline & MEP Late epoch & Intensity $\times$ age & 2.508 & 1.998 & 0.105 \\
\hline \multicolumn{6}{|l|}{ Gender } \\
\hline \multirow[t]{4}{*}{ Anodal stimulation } & MEP Early epoch $_{\text {E }}$ & Intensity & 4 & 3.529 & $0.011^{*}$ \\
\hline & MEP Early epoch & Intensity $\times$ gender & 4 & 0.504 & 0.733 \\
\hline & MEP Late epoch & Intensity & 4 & 1.178 & 0.328 \\
\hline & MEP $_{\text {Late epoch }}$ & Intensity $\times$ gender & 4 & 0.840 & 0.504 \\
\hline \multirow[t]{4}{*}{ Cathodal stimulation } & MEP $_{\text {Early epoch }}$ & Intensity & 4 & 2.432 & 0.056 \\
\hline & MEP Early epoch & Intensity $\times$ gender & 4 & 1.195 & 0.321 \\
\hline & MEP Late epoch & Intensity & 2.456 & 1.881 & 0.125 \\
\hline & MEP & Intensity $\times$ gender & 2.456 & 0.537 & 0.625 \\
\hline
\end{tabular}

Repeated-measures ANOVAs with the repeated factor of intensity were calculated for subject-specific covariates of subject average baseline $S I_{1 \mathrm{mV}}$ (stimulus intensity for $1 \mathrm{mV}$ amplitude), age, and gender, against grand averaged MEPs from time bins of either the first 0-30 min (Early epoch) or the final 60-120 min (Late epoch) after tDCS stimulation. *Significant effects (where $P<0.05$ ). A main interaction effect was only observed for the factor of Intensity $\times \mathrm{SI}_{1 \mathrm{mv}}$ for the anodal stimulation group.

Table 4. Correlation between $\mathrm{Sl}_{1 \mathrm{mv}}$ and $\mathrm{MEP}$ Early epoch

\begin{tabular}{|c|c|c|c|}
\hline & & Pearson coefficient $r$ & $P$ value \\
\hline \multirow[t]{5}{*}{ Anodal stimulation } & Sham & -0.142 & 0.551 \\
\hline & $0.5 \mathrm{~mA}$ & -0.182 & 0.442 \\
\hline & $1.0 \mathrm{~mA}$ & -0.474 & $0.035^{*}$ \\
\hline & $1.5 \mathrm{~mA}$ & 0.405 & 0.076 \\
\hline & $2.0 \mathrm{~mA}$ & 0.081 & 0.773 \\
\hline \multirow[t]{5}{*}{ Cathodal stimulation } & Sham & -0.032 & 0.900 \\
\hline & $0.5 \mathrm{~mA}$ & 0.354 & 0.150 \\
\hline & $1.0 \mathrm{~mA}$ & -0.042 & 0.869 \\
\hline & $1.5 \mathrm{~mA}$ & -0.287 & 0.248 \\
\hline & $2.0 \mathrm{~mA}$ & -0.277 & 0.267 \\
\hline
\end{tabular}

Pearson's correlation coefficient was calculated for each intensity using each subject's respective $S 11 \mathrm{mV}$ as one variable and the grand-averaged, normalized MEP from the early epoch (0-30 min post stimulation) as the second variable. A significant negative correlation was observed for $1.0 \mathrm{~mA}$ anodal stimulation only. plasticity. Further, we observed that individual sensitivity to TMS may be an important covariate for anodal tDCS efficacy. Below, we discuss possible underlying mechanisms behind our main findings in light of previous studies in the field.

\section{No differences between anodal stimulation} intensities

Overall, higher anodal intensities did not significantly differ from lower intensities over the whole group and time course, which is in accordance with two reported studies. Kidgell et al. (2013) observed that $0.8 \mathrm{~mA}$, $1.0 \mathrm{~mA}$ and $1.2 \mathrm{~mA}\left(25 \mathrm{~cm}^{2}\right.$ electrodes, $10 \mathrm{~min}$ stimulation) resulted in identical excitability after-effects as measured by MEP size as well as short-latency intracortical inhibition (SICI), suggesting that inhibitory neurons may be non-differentially involved in facilitating cortical excitability. Here, intracortical measures were not obtained and the intensity range was much larger; thus, the extent to which inhibitory circuits may play a role

(Q) 2016 The Authors. The Journal of Physiology @ 2016 The Physiological Society 
at these intensities is unclear and may be of interest in further studies. Bastani \& Jaberzadeh (2013) reported uniform effects of 0.3 and $2.0 \mathrm{~mA}$ anodal tDCS on cortical excitability after effects ( $10 \mathrm{~min}$ duration, $24 \mathrm{~cm}^{2} / 35 \mathrm{~cm}^{2}$ target/reference electrodes), and proposed the role of voltage-gated calcium channels driving the effect at lower current intensities, since these channels have lower voltage-dependent thresholds compared to NMDA or $\alpha$-amino-3-hydroxy-5-methyl-4-isoxazolepropionic acid (AMPA) receptors, which, along with calcium channels, have been identified to be relevant for plasticity induction via tDCS (Liebetanz et al. 2002; Nitsche et al. 2003a, 2004). Over the whole group, anodal stimulation at higher intensities did not result in significantly greater effects than lower intensities, which lends credence to the hypothesis of homeostatic counter-regulation limiting
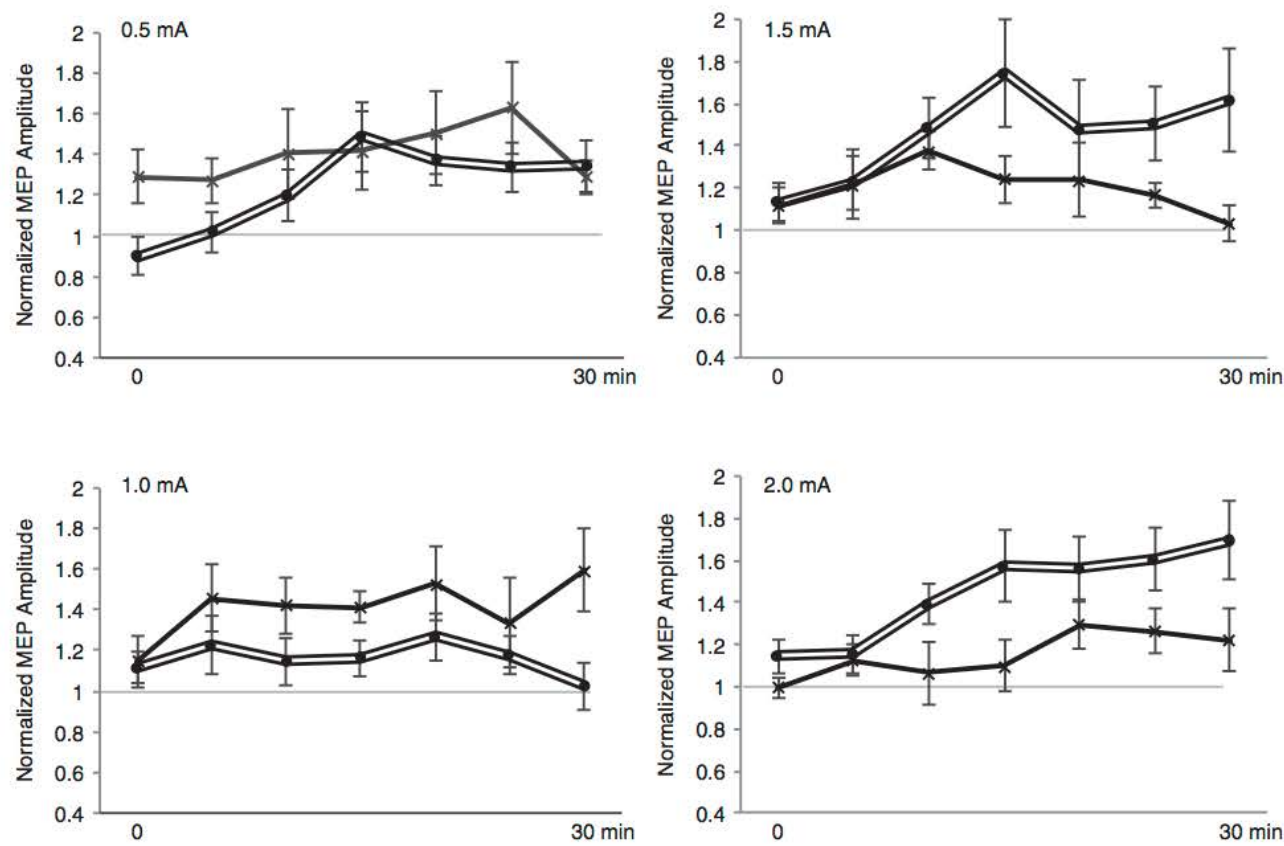

over-excitation as observed in similar human and animal studies (Rioult-Pedotti et al. 2007; Pozo \& Goda, 2010; Krause et al. 2013; Monte-Silva et al. 2013). However, the lack of clearly marked differences in DC amperage in the present exploratory study should not be interpreted to mean amperage has no effect. Other tDCS parameters, such as stimulation duration and electrode montage may potentially interact with each other, possibly resulting in non-linear effects. With regard to the electrode montage, the present study employed the conventionally used 'M1-contralateral superior frontal orbit' arrangement, with an enlarged reference electrode $\left(100 \mathrm{~cm}^{2}\right)$ as it was previously shown to reduce unwanted physiological effects, at least up to a $1.0 \mathrm{~mA}$ setting (Nitsche et al. 2007). Further studies would be required to compare the conventional montage with other montages which use

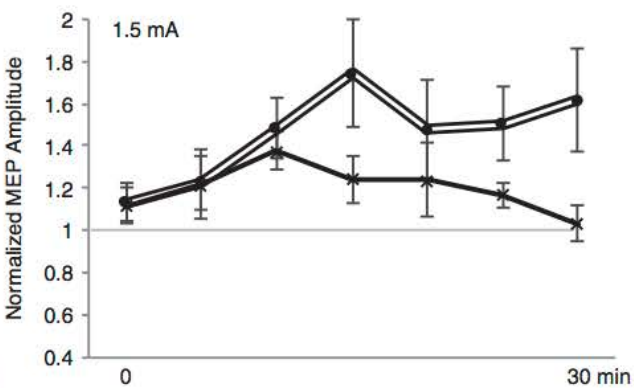

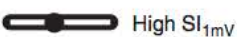

Figure 6. Inter-individual differences in cortical excitability modulation following anodal tDCS

Post anodal tDCS time course ( $0-30 \mathrm{~min})$ differences and trends between median-split groups of low and high thresholds to TMS, based on the stimulus intensity for $1 \mathrm{mV}$ amplitude $\left(S I_{1 \mathrm{mV}}\right)$. Error bars represent standard error. At lower intensities of $0.5 \mathrm{~mA}$ and $1.0 \mathrm{~mA}$, subjects with lower $\mathrm{Sl}_{1 \mathrm{mv}}$ showed greater effects in cortical excitability facilitation whereas with higher intensities of $1.5 \mathrm{~mA}$ and $2.0 \mathrm{~mA}$, subjects with higher $\mathrm{SI}_{1 \mathrm{mv}}$ responded with a greater change in excitability compared to the Low Sl $1 \mathrm{mv}$ subjects. Notice that an upward trend of excitability facilitation was observed for subjects with higher Slinv which was more pronounced at higher intensities, although this three-way interaction could not be inferred as significant: $F_{(18,1045)}=1.281, P=0.198$. 
multiple small electrodes in concentric ring arrangements, as these have been shown to induce a more focused electric field, and also result in slightly enhanced effects in motor cortical excitability (Datta et al. 2009; Kuo et al. 2013).

\section{Reproducibility and variability of tDCS effects on cortical excitability}

A recent study by Chew et al. (2015) investigating cortical excitability after M1 anodal tDCS (10 min duration, $16 \mathrm{~cm}^{2}$ target/reference electrodes) did not observe a main effect of intensity, although no sham condition was tested. Moreover, intra-individual reliability of $0.5 \mathrm{~mA}$ over the $30 \mathrm{~min}$ following stimulation was reported to be poor $(\operatorname{ICC}(2,1)=-0.50)$, and it was further reported that participants responded strongly to either $0.2 \mathrm{~mA}$ or $2.0 \mathrm{~mA}$, only. A study from Lopez-Alonso et al. (2015) investigating $1.0 \mathrm{~mA}$ anodal tDCS (13 min duration, $35 \mathrm{~cm}^{2}$ target/reference electrodes) reported good intra-individual reliability of anodal $1.0 \mathrm{~mA}$ tDCS over the first $30 \mathrm{~min}(\operatorname{ICC}(2,1)=0.565)$, although measurements obtained during the $30 \mathrm{~min}$ afterwards showed poorer reliability $(\operatorname{ICC}(2,1)=-0.028)$. The present findings of intra-individual reliability in $1.0 \mathrm{~mA}$ anodal tDCS show stronger reliability, both over early and late measurement periods $(\operatorname{ICC}(2,1)=0.74$ and 0.64 , between $0-30$ and $60-120 \mathrm{~min}$, respectively). The discrepancy between the present results and previous reports may possibly be due to the smaller sample size tested here $(n=7)$. However, we note that whereas the previous studies assessed re-test reliability over two sessions, the present study collected data over three sessions, and over a longer period of monitoring (120 min). Previous studies have identified various possible sources of intra-individual variability in the induced response to stimulation protocols, which include such factors as

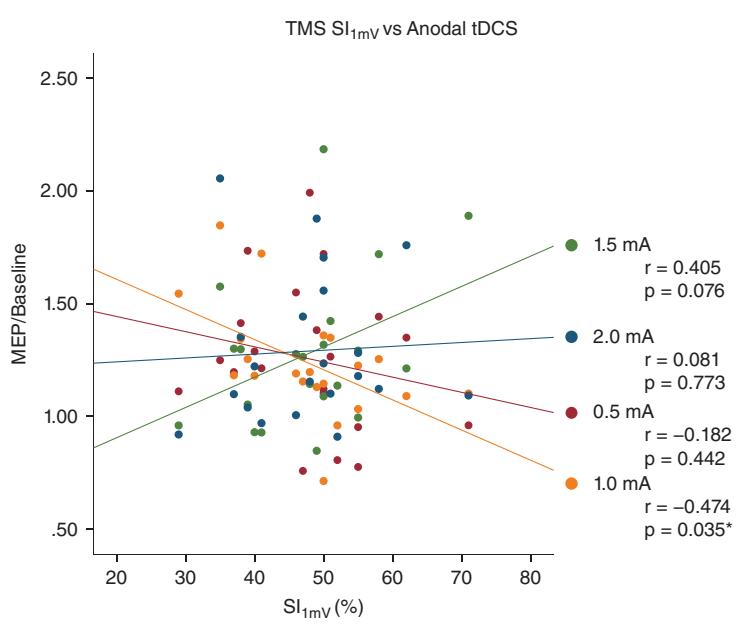

attention level, time of the day, and hormonal fluctuations (see Ridding \& Ziemann, 2010 for a review). Most of these factors, however, can be controlled for with adequate sample sizes or factored into the statistical analysis if appropriately documented and reported. Another possible reason for low reliability may be due in part to elevated anxiety associated with participants naive to stimulation inducing protocols which may affect cortical excitability (Wassermann et al. 2001) (for example, due to the loud sounds and novel sensations of the stimulation, similar to elevated heart rates during the start of MRI investigations; van Minde et al. 2013). In an attempt to control for these factors, all participants in the study first attended a preliminary session to experience sensation of TMS test pulses. To further ensure stability in the motor-cortical excitability, participants were seated in a relaxed manner in the laboratory for at least $10 \mathrm{~min}$ before the start of the experiment. However, the full extent of the within-subject variation in cortical excitability, and whether our additional testing conditions were effective in reducing the non-stability, is unknown and remains to be further probed in future studies.

In addition to intra-individual sources of variability, it is also important to consider between-individual sources, as they may contribute the most to the total variance (López-Alonso et al. 2015). A previous study by Wiethoff et al. (2014) reported a correlation between anodal tDCS efficacy and the MEP latency difference between monophasic anterior-posterior induced currents and latero-medial induced currents. The researchers thus proposed the role of early I-wave recruitment in facilitating tDCS response, which appears to be evident in other brain stimulation protocols, such as TBS (Hamada et al. 2013). In the present study, we observed that sensitivity to TMS $\left(\mathrm{SI}_{1 \mathrm{mV}}\right)$ tended to correlate with anodal tDCS efficacy in an intensity-dependent manner. With lower intensities,
Figure 7. Relationship between individual TMS SI $I_{1 \mathrm{mV}}$ sensitivity and efficacy of anodal tDCS on cortical excitability

For each active anodal tDCS intensity, each individual's grand-averaged response over 0-30 min following stimulation was plotted as a function of his/her baseline TMS SI $1 \mathrm{mv}$ (stimulus intensity for $1 \mathrm{mV}$ amplitude). A negative correlation was observed with $1.0 \mathrm{~mA}$ anodal $\mathrm{tDCS}(r=-0.474, P=0.035)$ [Colour figure can be viewed at wileyonlinelibrary.com] 
participants who had relatively higher sensitivity to TMS tended to respond with greater after-effects in excitability, a finding which was also observed in a previous report (Labruna et al. 2015). In comparison, our analysis showed the largest effect in sensitivity with $1.0 \mathrm{~mA}$ where the split-group effect size was $d=0.91$ and the absolute correlation between $\mathrm{SI}_{1 \mathrm{mV}}$ and $1.0 \mathrm{~mA}$ after-effects was $r=-0.47$ (i.e. $22.1 \%$ of the estimated variance explained by $\mathrm{SI}_{1 \mathrm{mV}}$, uncorrected $P=0.035$; Fig. 7) which is marginally higher than the findings from Labruna et al. (2015) of 36 subjects where the $1.0 \mathrm{~mA}$ correlation $r=-0.20$ (accounting for $4 \%$ of estimated variance) One explanation for these findings could be anatomical variability affecting the path of the current into the skull. Previous TMS studies found that the TMS motor threshold correlated with the coil-to-cortex distance (Kozel et al. 2000; McConnell et al. 2001; Herbsman et al. 2009) and modelling studies have shown that higher tDCS intensities should induce higher electric fields in the cortex, although these may be nuanced by skull thickness and composition (Datta et al. 2012; Opitz et al. 2015). With higher tDCS intensities over $1.0 \mathrm{~mA}$, it may be possible that mechanisms of homeostatic counter-regulation may have limited the effects of $\mathrm{tDCS}$ for subjects with lower $\mathrm{SI}_{1 \mathrm{mV}}$, whereas subjects with higher $\mathrm{SI}_{1 \mathrm{mV}}$ were in the necessary intensity range for positive effects. This hypothesis is partially supported from a group-wise comparison of the first 30 min post-stimulation time series of excitability changes: subjects with lower $\mathrm{SI}_{1 \mathrm{mV}}$ tended to reach maximum excitability approximately $15-20 \mathrm{~min}$ post stimulation and then returned to baseline (in the case of $1.5 \mathrm{~mA}$ ) or plateaued $(2.0 \mathrm{~mA})$ for the remainder of the monitoring (Fig. $6 C$ and $D$ ). Subjects with higher $\mathrm{SI}_{1 \mathrm{mV}}$, however, tended towards steadily increasing excitability. The precise mechanisms behind this delayed but increasing effect, also observed in previous reports with $2.0 \mathrm{~mA}$ tDCS (Batsikadze et al. 2013; Kuo et al. 2013) remain unknown and should be investigated further.

A clear association between TMS sensitivity and cathodal tDCS was not observed, possibly due to the limited range of intensities required to induce cortico-spinal excitability diminution.

\section{Cathodal stimulation at higher intensities reduced after-effects}

Increasing cathodal intensities did not yield greater effects. DC intensities of $0.5 \mathrm{~mA}$ and $1.0 \mathrm{~mA}$ led to excitability diminution, which was not achieved by $1.5 \mathrm{~mA}$ and $2.0 \mathrm{~mA}$. The effects of the lower intensities are replications of results of respective previous studies of $1.0 \mathrm{~mA}$ cathodal tDCS ( 9 min duration, $35 \mathrm{~cm}^{2}$ electrodes: Nitsche et al. 2003b; $18 \mathrm{~min}$ duration: Monte-Silva et al. 2010; 20 min duration, $35 \mathrm{~cm}^{2} / 100 \mathrm{~cm}^{2}$ target/reference electrodes: Batsikadze et al. 2013) as well as one study of
$0.3 \mathrm{~mA}\left(20 \mathrm{~min}\right.$ duration $3 \mathrm{~cm}^{2} / 12 \mathrm{~cm}^{2}$ target/reference electrode: Vaseghi et al. 2015). Notably, intensities higher than $1.0 \mathrm{~mA}$ did not result in a reduction of cortical excitability, as has also been recently reported in similar studies. Batsikadze et al. (2013) showed that $20 \mathrm{~min}$ of $2.0 \mathrm{~mA}$ cathodal tDCS $\left(35 \mathrm{~cm}^{2} / 100 \mathrm{~cm}^{2}\right.$ target/reference electrodes) shifted cortical plasticity from diminution to facilitation. Wiethoff et al. (2014) investigated $10 \mathrm{~min}$ of $2.0 \mathrm{~mA}$ cathodal stimulation ( $35 \mathrm{~cm}^{2}$ electrodes), finding variable and ultimately non-conclusive effects. However, another $10 \mathrm{~min}, 2.0 \mathrm{~mA}$ cathodal tDCS study observed a reduction in cortical excitability, where the peak effects were observed after 30-60 $\mathrm{min}$ (Kuo et al. 2013). In a study on adolescents, $10 \mathrm{~min}$ of $0.5 \mathrm{~mA}$ cathodal tDCS ( $35 \mathrm{~cm}^{2}$ electrodes) significantly decreased cortical excitability but $1.0 \mathrm{~mA}$ cathodal tDCS led to an increase (Moliadze et al. 2015). This study supports the general concept of non-linear intensity-dependent effects of cathodal tDCS. The different turning point in this specific population, as compared to adults, might be caused by anatomical or physiological differences between these age groups, which could result in a more effective stimulation of the developing brain with identical stimulation intensity. Putative hypotheses for the reversed or negated effects, which have also been observed for other brain stimulation protocols (e.g. theta-burst stimulation: Doeltgen \& Ridding, 2011; tACS, and tRNS: Moliadze et al. 2012) have pointed to the bi-directional effects of calcium influx caused by stimulation, whereby low postsynaptic calcium causes long-term depression and larger calcium concentrations (e.g. due to stronger stimulation intensity) result in long-term potentiation (Cho et al. 2001; Lisman, 2001). Our findings support this concept and suggest that intensities around $1.0 \mathrm{~mA}$ might be optimal in inducing the strongest inhibition of motor-cortical excitability in healthy adults.

\section{Conclusion}

Our main finding that stimulation at higher intensities does not yield correspondingly greater after-effects partially confirms previous studies. Anodal stimulation intensities from 0.5 to $2.0 \mathrm{~mA}$ and cathodal stimulation of $1.0 \mathrm{~mA}$ resulted in significant after-effects in excitability facilitation and diminution, respectively. Whether higher current intensities or longer stimulation duration, perhaps following intervals (Monte-Silva et al. 2010, 2013) would produce greater or prolonged corticospinal excitability effects cannot be concluded, and should be the topic of future studies. Moreover, whether effects were localized to only the target region cannot be concluded as tDCS over the motor cortex also affects functionally connected cortical and sub-cortical areas (Polanía et al. $2011 a, b)$. Obtaining a more intricate physiological understanding of tDCS, especially when TMS may not be 
particularly suitable, may require other available means of investigation, such as within the neuroimaging or cognitive neuroscience domains. We also observed that inter-individual differences in sensitivity to TMS may be an important covariate factor of anodal tDCS, but our findings require further replications with more extensive measurements. For example, our measure of sensitivity was the $\mathrm{SI}_{1 \mathrm{mV}}$, which corresponds to approximately $130-140 \%$ of the resting motor threshold (Groppa et al. 2012). A better understanding of the relationship between TMS susceptibility and tDCS efficacy would require further studies that assess the correlation of the slope as well as different points on the TMS-MEP recruitment curve with tDCS efficacy, and at different current intensities. Finally, we recognize that while the prospect of individualized stimulation protocols, based on TMS latency, sensitivity or similar metric is an intriguing concept, it is not self-evident that the findings here, observed on a cohort of healthy and young adults, translate one-to-one to elderly or clinical populations. Given the results of a cathodal tDCS titration study in children and adolescents (Moliadze et al. 2015), as well as significant differences in neurotransmitter availability and corticospinal excitability across various neuropsychiatric states (Bunse et al. 2014), this is not just a theoretical limitation and requires important consideration for future studies.

\section{References}

Ambrus GG, Al-Moyed H, Chaieb L, Sarp L, Antal A \& Paulus W (2012). The fade-in - short stimulation - fade out approach to sham $\mathrm{tDCS}$ - reliable at $1 \mathrm{~mA}$ for naïve and experienced subjects, but not investigators. Brain Stimul 5, 499-504.

Ambrus GG, Paulus W \& Antal A (2010). Cutaneous perception thresholds of electrical stimulation methods: Comparison of tDCS and tRNS. Clin Neurophysiol 121, 1908-1914.

Bashir S, Perez JM, Horvath JC, Pena-Gomez C, Vernet M, Capia A, Alonso-Alonso M \& Pascual-Leone A (2014). Differential effects of motor cortical excitability and plasticity in young and old individuals: A transcranial magnetic stimulation (TMS) study. Front Aging Neurosci 6, 1-13.

Bastani A \& Jaberzadeh S (2013). Differential modulation of corticospinal excitability by different current densities of anodal transcranial direct current stimulation. PLoS One 8, e72254

Batsikadze G, Moliadze V, Paulus W, Kuo MF \& Nitsche MA (2013). Partially non-linear stimulation intensity-dependent effects of direct current stimulation on motor cortex excitability in humans. J Physiol 591, 1987-2000.

Boggio P, Khoury L, Martins D, Martins O, de Macedo E \& Fregni F (2009). Temporal cortex direct current stimulation enhances performance on a visual recognition memory task in Alzheimer disease. J Neurol Neurosurg Psychiatry 80, $444-4447$.
Brunoni AR, Valiengo L, Baccaro A, Zanão TA, de Oliveira JF, Goulart A, Boggio PS, Lotufo PA, Benseñor IM \& Fregni F (2013). The sertraline vs. electrical current therapy for treating depression clinical study: results from a factorial, randomized, controlled trial. JAMA Psychiatry 70, 383-391.

Bunse T, Wobrock T, Strube W, Padberg F, Palm U, Falkai P \& Hasan A (2014). Motor cortical excitability assessed by transcranial magnetic stimulation in psychiatric disorders: a systematic review. Brain Stimul 7, 158-169.

Chew T, Ho K-A \& Loo CK (2015). Inter- and intra-individual variability in response to transcranial direct current stimulation (tDCS) at varying current intensities. Brain Stimul 1-8.

Cho K, Aggleton JP, Brown MW \& Bashir ZI (2001). An experimental test of the role of postsynaptic calcium levels in determining synaptic strength using perirhinal cortex of rat. JPhysiol 532, 459-466.

Cicchetti DV (1994). Guidelines, criteria, and rules of thumb for evaluating normed and standardized assessment instruments in psychology. Psychol Assess 6, 284-290.

Datta A, Bansal V, Diaz J, Patel J, Reato D \& Bikson M (2009). Gyri-precise head model of transcranial direct current stimulation: Improved spatial focality using a ring electrode versus conventional rectangular pad. Brain Stimul 2, 201-207.

Datta A, Truong D, Minhas P, Parra LC \& Bikson M (2012). Inter-individual variation during transcranial direct current stimulation and normalization of dose using MRI-derived computational models. Front Psychiatry 3, 1-8.

Doeltgen SH \& Ridding MC (2011). Low-intensity, short-interval theta burst stimulation modulates excitatory but not inhibitory motor networks. Clin Neurophysiol 122, 1411-1416.

Flöel A (2014). TDCS-enhanced motor and cognitive function in neurological diseases. Neuroimage 85, 934-947.

Gandiga PC, Hummel FC \& Cohen LG (2006). Transcranial DC stimulation (tDCS): A tool for double-blind sham-controlled clinical studies in brain stimulation. Clin Neurophysiol 117, 845-850.

Groppa S, Oliviero A, Eisen A, Quartarone A, Cohen LG, Mall V, Kaelin-Lang A, Mima T, Rossi S, Thickbroom GW, Rossini PM, Ziemann U, Valls-Solé J \& Siebner HR (2012). A practical guide to diagnostic transcranial magnetic stimulation: Report of an IFCN committee. Clin Neurophysiol 123, 858-882.

Guleyupoglu B, Febles N, Minhas P, Hahn C \& Bikson M (2014). Reduced discomfort during high-definition transcutaneous stimulation using $6 \%$ benzocaine. Front Neuroeng 7, 28.

Hamada M, Murase N, Hasan A, Balaratnam M \& Rothwell JC (2013). The role of interneuron networks in driving human motor cortical plasticity. Cereb Cortex 23, 1593-1605.

Herbsman T, Forster L, Molnar C, Dougherty R, Christie D, Koola J, Ramsey D, Morgan PS, Bohning DE, George MS \& Nahas Z (2009). Motor threshold in transcranial magnetic stimulation: The impact of white matter fiber orientation and skull-to-cortex distance. Hum Brain Mapp 30, 2044-2055.

(Q) 2016 The Authors. The Journal of Physiology $₫ 2016$ The Physiological Society 
Jennum P, Winkel H \& Fuglsang-Frederiksen A (1995). Repetitive magnetic stimulation and motor evoked potentials. Electroencephalogr Clin Neurophysiol Mot Control 97, 96-101.

Kidgell DJ, Daly RM, Young K, Lum J, Tooley G, Jaberzadeh S, Zoghi M \& Pearce AJ (2013). Different current intensities of anodal transcranial direct current stimulation do not differentially modulate motor cortex plasticity. Neural Plast 2013, 1-9.

Kozel FA, Nahas ZH, DeBrux C, Molloy M, Lorberbaum JP, Bohning DE, Risch SC \& George MS (2000). How coil-cortex distance relates to age, motor threshold, and antidepressant response to repetitive transcranial magnetic stimulation. J Neuropsychiatry Clin Neurosci 12, 376-384.

Krause B, Márquez-Ruiz J \& Cohen Kadosh R (2013). The effect of transcranial direct current stimulation: a role for cortical excitation/inhibition balance? Front Hum Neurosci 7, 602.

Kuo H-I, Bikson M, Datta A, Minhas P, Paulus W, Kuo M-F \& Nitsche MA (2013). Comparing cortical plasticity induced by conventional and high-definition $4 \times 1$ ring tDCS: A neurophysiological study. Brain Stimul 6, 644-648.

Kuo M-F \& Nitsche MA (2012). Effects of transcranial electrical stimulation on cognition. Clin EEG Neurosci 43, 192-199.

Kuo M-F, Paulus W \& Nitsche MA (2006). Sex differences in cortical neuroplasticity in humans. Neuroreport 17 , 1703-1707.

Kuo M-F, Paulus W \& Nitsche MA (2014). Therapeutic effects of non-invasive brain stimulation with direct currents (tDCS) in neuropsychiatric diseases. Neuroimage 85 , 948-960.

Labruna L, Jamil A, Fresnoza S, Batsikadze G, Kuo M-F, Vanderschelden B, Ivry RB \& Nitsche MA (2015). Efficacy of anodal transcranial direct current stimulation is related to sensitivity to transcranial magnetic stimulation. Brain Stimul 9, 8-15

Liebetanz D, Nitsche MA, Tergau F \& Paulus W (2002). Pharmacological approach to the mechanisms of transcranial DC-stimulation-induced after-effects of human motor cortex excitability. Brain 125, 2238-2247.

Lisman JE (2001). Three $\mathrm{Ca}^{2+}$ levels affect plasticity differently: The LTP zone, the LTD zone and no man's land. J Physiol 532, 285.

López-Alonso V, Cheeran B, Río-Rodríguez D \& Fernández-Del-Olmo M (2014). Inter-individual variability in response to non-invasive brain stimulation paradigms. Brain Stimul 7, 372-380.

López-Alonso V, Fernández-del-Olmo M, Costantini A, Gonzalez-Henriquez JJ \& Cheeran B (2015). Intra-individual variability in the response to anodal transcranial direct current stimulation. Clin Neurophysiol 126, 2342-2347.

McConnell KA, Nahas Z, Shastri A, Lorberbaum JP, Kozel FA, Bohning DE \& George MS (2001). The transcranial magnetic stimulation motor threshold depends on the distance from coil to underlying cortex: A replication in healthy adults comparing two methods of assessing the distance to cortex. Biol Psychiatry 49, 454-459.

McFadden JL, Borckardt JJ, George MS \& Beam W (2011). Reducing procedural pain and discomfort associated with transcranial direct current stimulation. Brain Stimul 4, $38-42$.

(c) 2016 The Authors. The Journal of Physiology @ 2016 The Physiological Society
Moliadze V, Atalay D, Antal A \& Paulus W (2012). Close to threshold transcranial electrical stimulation preferentially activates inhibitory networks before switching to excitation with higher intensities. Brain Stimul 5, 505-511.

Moliadze V, Schmanke T, Andreas S, Lyzhko E, Freitag CM \& Siniatchkin $M$ (2015). Stimulation intensities of transcranial direct current stimulation have to be adjusted in children and adolescents. Clin Neurophysiol 126, 1392-1399.

Monte-Silva K, Kuo M-F, Hessenthaler S, Fresnoza S, Liebetanz D, Paulus W \& Nitsche MA (2013). Induction of late LTP-like plasticity in the human motor cortex by repeated non-invasive brain stimulation. Brain Stimul 6, 424-432.

Monte-Silva K, Kuo M-F, Liebetanz D, Paulus W \& Nitsche M (2010). Shaping the optimal repetition interval for cathodal transcranial direct current stimulation (tDCS).

J Neurophysiol 103, 1735-1740.

Nitsche MA, Cohen LG, Wassermann EM, Priori A, Lang N, Antal A, Paulus W, Hummel F, Boggio PS, Fregni F \& Pascual-Leone A (2008). Transcranial direct current stimulation: State of the art 2008. Brain Stimul 1, 206-223.

Nitsche MA, Doemkes S, Karakose T, Antal A, Liebetanz D, Lang N, Tergau F, Paulus W, Karaköse T, Antal A, Liebetanz D, Lang N, Tergau F \& Paulus W (2007). Shaping the effects of transcranial direct current stimulation of the human motor cortex. J Neurophysiol 97, 3109-3117.

Nitsche MA, Fricke K, Henschke U, Schlitterlau A, Liebetanz D, Lang N, Henning S, Tergau F \& Paulus W (2003a). Pharmacological modulation of cortical excitability shifts induced by transcranial direct current stimulation in humans. J Physiol 553, 293-301.

Nitsche MA, Liebetanz D, Schlitterlau A, Henschke U, Fricke K, Frommann K, Lang N, Henning S, Paulus W \& Tergau F (2004). GABAergic modulation of DC stimulation-induced motor cortex excitability shifts in humans. Eur J Neurosci 19, 2720-2726.

Nitsche MA, Nitsche MS, Klein CC, Tergau F, Rothwell JC \& Paulus W (2003b). Level of action of cathodal DC polarisation induced inhibition of the human motor cortex. Clin Neurophysiol 114, 600-604.

Nitsche MA \& Paulus W (2000). Excitability changes induced in the human motor cortex by weak transcranial direct current stimulation. J Physiol 527, 633-639.

Nitsche MA \& Paulus W (2001). Sustained excitability elevations induced by transcranial DC motor cortex stimulation in humans. Neurology 57, 1899-1901.

Oldfield RC (1971). The assessment and analysis of handedness: The Edinburgh inventory. Neuropsychologia 9, 97-113.

Opitz A, Paulus W, Will A \& Thielscher A (2015). Anatomical determinants of the electric field during transcranial direct current stimulation. Neuroimage 109, 2.

Pascual-Leone A, Valls-sole J, Wassermann EM \& Hallett M (1994). Responses to rapid-rate transcranial magnetic stimulation of the human motor cortex. Brain 117, 847-858.

Polanía R, Nitsche MA \& Paulus W (2011a). Modulating functional connectivity patterns and topological functional organization of the human brain with transcranial direct current stimulation. Hum Brain Mapp 32, 1236-1249. 
Polanía R, Paulus W \& Nitsche MA (2011b). Modulating cortico-striatal and thalamo-cortical functional connectivity with transcranial direct current stimulation. Hum Brain Mapp 32, 1236-1249.

Pozo K \& Goda Y (2010). Unraveling mechanisms of homeostatic synaptic plasticity. Neuron $66,337-351$.

Ridding MC \& Ziemann U (2010). Determinants of the induction of cortical plasticity by non-invasive brain stimulation in healthy subjects. J Physiol 588, 2291-2304.

Rioult-Pedotti M-S, Donoghue JP \& Dunaevsky A (2007).

Plasticity of the synaptic modification range. J Neurophysiol 98, 3688-3695.

Shekhawat GS, Stinear CM \& Searchfield GD (2013). Transcranial direct current stimulation intensity and duration effects on tinnitus suppression. Neurorehabil Neural Repair 27, 164-172.

Shin Y-I, Foerster Á \& Nitsche MA (2015). Transcranial direct current stimulation ( $\mathrm{tDCS}$ ) - application in

neuropsychology. Neuropsychologia $69,154-175$.

Shrout PE \& Fleiss JL (1979). Intraclass correlations: Uses in assessing rater reliability. Psychol Bull 86, 420-428.

Stagg CJ \& Nitsche MA (2011). Physiological basis of transcranial direct current stimulation. Neurosci 17, 37-53.

Stefan K, Kunesch E, Cohen LG, Benecke R \& Classen J (2000). Induction of plasticity in the human motor cortex by paired associative stimulation. Brain 123, 572-584.

Strube W, Bunse T, Malchow B \& Hasan A (2015). Efficacy and interindividual variability in motor-cortex plasticity following anodal $\mathrm{tDCS}$ and paired-associative stimulation. Neural Plast 2015, 1-10.

van Minde D, Klaming L \& Weda H (2013). Pinpointing moments of high anxiety during an MRI examination. Int $J$ Behav Med 1-9.

Vaseghi B, Zoghi M \& Jaberzadeh S (2015). Differential effects of cathodal transcranial direct current stimulation of prefrontal, motor and somatosensory cortices on cortical excitability and pain perception - a double-blind randomised sham-controlled study. Eur J Neurosci 42, 2426-2437.

Wassermann EM, Greenberg BD, Nguyen MB \& Murphy DL (2001). Motor cortex excitability correlates with an anxiety-related personality trait. Biol Psychiatry 50, 377-382.
Wiethoff S, Hamada M \& Rothwell JC (2014). Variability in response to transcranial direct current stimulation of the motor cortex. Brain Stimul 7, 468-475.

Woods AJ, Antal A, Bikson M, Boggio PS, Brunoni AR, Celnik P, Cohen LG, Fregni F, Herrmann CS, Kappenman ES, Knotkova H, Liebetanz D, Miniussi C, Miranda PC, Paulus W, Priori A, Reato D, Stagg C, Wenderoth N \& Nitsche MA (2015). A technical guide to tDCS, and related non-invasive brain stimulation tools. Clin Neurophysiol 127, 1031-1048.

\section{Additional information}

\section{Competing interests}

The authors declare no competing financial interests.

\section{Author contributions}

The experiments were conducted at the University Medical Centre, Department of Clinical Neurophysiology, Georg-August University, Göttingen. M.A.N., W.P., G.B. and H.I.K. contributed to the conception and design of the experiment. A.J., H.I.K., G.B., L.L., A.H. and M.A.N. contributed to the collection, analysis and interpretation of the data. A.J. drafted the paper and M.A.N., G.B., H.I.K, L.L., A.H. and W.P. revised it critically for important intellectual content. All authors approved the final version of the manuscript and agree to be accountable for all aspects of the work. All persons designated as authors qualify for authorship, and all those who qualify for authorship are listed.

\section{Funding}

This work was supported by a research grant from the German Federal Ministry of Education and Research (BMBF) (grant 'EYE-TSS', 03IPT605E).

\section{Acknowledgements}

We thank Dr Yuichiro Shirota for valuable comments and discussions. 
Current intensity- and polarity-dependent effects of transcranial direct current stimulation on cortical activation: an fMRI study

Title:

Current intensity- and polarity-specific online and aftereffects of transcranial direct current stimulation: an fMRI study

\section{Authors:}

Asif Jamil ${ }^{1,2}$, Giorgi Batsikadze ${ }^{3}$, Hsiao-I. Kuo ${ }^{1,2}$, Peter Dechent ${ }^{1}$, Walter Paulus ${ }^{1}$, Michael A. Nitsche ${ }^{2,4}$

${ }^{1}$ Department of Clinical Neurophysiology

University Medical Center, University of Göttingen

Göttingen 37075, Germany

${ }^{2}$ Leibniz Research Centre for Working Environment and Human Factors

Dortmund 44139, Germany

${ }^{3}$ Department of Neurology, Essen University Hospital, University of Duisburg-Essen

Essen, Germany

${ }^{4}$ University Medical Hospital Bergmannsheil

Bochum, 44789 Germany

\section{Corresponding author:}

Asif Jamil

Dept. Psychology and Neurosciences

Leibniz Research Centre for Working Environment and Human Factors

Ardeystr. 67

44139 Dortmund

Germany

phone: ++49 2311084301

email: jamil@ifado.de 


\title{
Highlights:
}

- Alterations in cerebral blood flow by varying intensities of tDCS were investigated

- Compared to sham, $1.0 \mathrm{~mA}$ and $2.0 \mathrm{~mA}$ cathodal tDCS decreased perfusion

- Anodal tDCS led to prolonged and intensity-dependent increases in perfusion

- Effects also directionally coincided with connectivity alterations to the targeted M1

\begin{abstract}
Transcranial direct current stimulation (tDCS) can effectively modulate a wide range of clinical and cognitive outcomes by modulating cortical excitability. Here, we systematically investigated anodal and cathodal tDCS-induced aftereffects on regional cerebral blood flow (rCBF) in healthy adult humans. 29 participants were enrolled in a sham-controlled repeated-measures study, during which sham, $0.5,1.0,1.5$ and $2.0 \mathrm{~mA}$ anodal or cathodal tDCS was delivered for $15 \mathrm{~min}$, using the target electrode $\left(35 \mathrm{~cm}^{2}\right)$ over the left primary motor cortex (M1) and the reference electrode $\left(100 \mathrm{~cm}^{2}\right)$ over the right supraorbital area. Time-course change in $\mathrm{rCBF}$ was measured as the mean cortical perfusion at 10 time-points before, during and up to $2 \mathrm{~h}$ following stimulation. As an additional assessment, regional perfusion-based corticocortical connectivity of left M1 was evaluated by means of a seed-based functional connectivity analysis. The results indicate a partially linear intensity and polarity-dependent relationship of tDCS on rCBF after-effects: all active intensities of anodal tDCS led to increased left M1 perfusion, where $2.0 \mathrm{~mA}$ tDCS resulted in the greatest increase when compared with sham (grandaverage $7.6 \%$ increase from baseline, $\mathrm{p}=0.017$ ). Additionally, $2.0 \mathrm{~mA}$ anodal tDCS further led to a polarity-specific rCBF increase across a large distribution of adjacent cortical areas, including the contralateral right M1. Cathodal tDCS over all active intensities led to a relatively modest decrease in left M1 perfusion from baseline, yet only $2.0 \mathrm{~mA}$ tDCS showed a significant decrease compared to sham (grand-average $7.2 \%$ decrease in rCBF vs. baseline, $\mathrm{p}=0.019$ ). Moreover, for both anodal and cathodal tDCS, a polarity-dependent regional functional connectivity modulation was observed with 1.0 and 2.0 $\mathrm{mA}$ tDCS, while $0.5 \mathrm{~mA}$ did not result in any significant modulatory effect. Inter-individual differences in baseline perfusion, gray matter volume, sensitivity to TMS, electrode to motor cortex distance, and age were not found to be significant predictors of rCBF aftereffects. Our findings provide, for the first time, evidence of lasting tDCS-induced alterations in arterial perfusion in the cerebral vascular system, which partially correlate with tDCS parameters in a polarity- and intensity-dependent way.
\end{abstract}

Keywords: transcranial Direct Current Stimulation, cerebral blood flow, current intensity, arterial spin labelling, motor cortex, inter-individual variability 


\subsection{Introduction}

Modulation of cortical neuroplasticity in humans- the process responsible for learning, memory and repair - stands as a critical learning objective in the fields of clinical neurology and cognitive neuroscience. Classic techniques, such as the use of extracellular recording electrodes in animal models and pharmacological modulation of central neurotransmitters in human models, have revealed substantial insights into mechanisms of long-term plasticity, such as the fundamental role of the synaptic glutamatergic system in inducing long-term potentiation (LTP) or long-term depression (LTD) (Cooke and Bliss, 2006; Lüscher and Malenka, 2012). Moreover, the recent developments of non-invasive brain stimulation methods have provided the attractive capability to bi-directionally modulate and probe these alterations at a system level in a safe and controlled manner (Bikson et al. 2016). One of the foremost techniques is transcranial direct current stimulation (tDCS), which has shown potential as it is inexpensive, well-tolerated, and suitable for a wide range of applications, for example in stroke rehabilitation, alleviation of depression, and even facilitation of working memory (Gandiga et al., 2006; Kuo et al., 2014; Nitsche et al., 2009; Polanía et al., 2012a; Shin et al., 2015).

tDCS is based on the application of low intensity DC stimulation for up to 30 min, and usually delivered via two or more electrodes placed on the scalp, targeting the cortical region of interest (Woods et al., 2016). Although clinical implementations of tDCS have shown promising efficacy, especially where used as adjunctive therapies (Brunoni et al., 2013; Lefaucheur et al., 2016), a deeper understanding of the precise mechanistic effects of tDCS remains warranted. Methodological probes to investigate physiological effects and after-effects of tDCS have primarily involved the use of transcranial magnetic stimulation (TMS), due its relative ease in assessing interventional modulations on motor cortico-spinal excitability (see Nitsche, 2011 for a review of these studies). Electroencephalography (EEG) studies have also shown after-effects of tDCS when applied to the motor cortex on neural oscillations (Notturno et al., 2014; Roy et al., 2014), as well as respective oscillatory-based functional connectivity alterations (Polanía et al., 2011a), revealing insights into larger neuroplastic composites of cortical activity. Further, neuroimaging methods, such as positron emission tomography (PET) and fMRI have shown widespread tDCS-induced network modulations of regional cerebral blood flow $(\mathrm{rCBF})$ and resting state functional connectivity (RSFC) (Lang et al., 2005; Polanía et al., 2012b, 2011b; Stagg et al., 2013; Zheng et al., 2011), although these studies have been limited to monitoring only short-term (up to $20 \mathrm{~min}$ ) after-effects of tDCS, whereas cortical excitability has been shown to be influenced for up to $1 \mathrm{~h}$ or more after stimulation cessation (Nitsche et al., 2008). Moreover, the long-term neuroplastic aftereffects of tDCS as observed from cortical excitability modulation may not necessarily be rooted to the same mechanisms as observed from effects in the cerebral vasculature. For example, earlier studies in which tDCS was combined with pharmacological modulations of central neurotransmitters uncovered the main role of the glutamatergic NMDA receptor in mediating long-term after- 
effects in cortical excitability (Liebetanz et al., 2002; Nitsche et al., 2008, 2003; Stagg and Nitsche, 2011). On the one hand, post-synaptic glutamate acting on neuronal NMDA receptors is also known to modulate cereberal blood flow, primarily through the $\mathrm{Ca}^{2+}$ dependent release of nitric oxide (NO), which subsequently acts on cyclic guanosine monophosphate (cGMP), resulting in arterial vasodilation, or secondarily through conversion of arachidonic acid to prostaglandins, which also dilate vessels (see Attwell et al., 2010, for a comprehensive review). On the other hand, several iontophoretic studies investigating non-vasoactive substances have documented "non-specific" vasodilation from anodal and cathodal current (Asberg et al., 1999; S Durand et al., 2002; Morris et al., 1995), which have been assumed to result from the action of prostaglandins via nociceptive nerve fibers, or possibly even from non-neuronal origins such as local changes in membrane potential or skin $\mathrm{pH}$ (Berliner, 1997; S. Durand et al., 2002). Thus, it remains unclear to which degree the previously reported $\mathrm{rCBF}$ and RSFC effects of tDCS are associated with the multi-regulatory mechanisms of the brain's neurovascular units (Iadecola, 2004).

A secondary, methodological objective has been to optimize tDCS parameters in order to achieve stable and longer-lasting effects. Interestingly, studies on the healthy population have thus far uncovered non-linear relationships of stimulation parameters, such as increasing the current strength (Batsikadze et al., 2013) or increasing stimulation duration (Monte-Silva et al., 2013, 2010). Inter-individual response variation to tDCS also remains an important issue (Chew et al., 2015; López-Alonso et al., 2014; Strube et al., 2015; Wiethoff et al., 2014), where factors such as cortical anatomy, genetics, and demographics may relevantly affect an individual's dose-response relationship. A recent analysis also uncovered TMS baseline-sensitivity as a potential covariate to efficacy of $1.0 \mathrm{~mA}$ anodal tDCS on greater modulation of cortical excitability, but not at higher intensities (Jamil et al., 2016; Labruna et al., 2016). A withinsubject neurophysiological investigation of these factors, and with respect to stimulation parameters, remains to be attained.

In the present study, we systematically investigated the effects of five anodal and cathodal tDCS intensities on cerebral blood flow (rCBF), over a timecourse of $2 \mathrm{~h}$. The first objective was to assess whether rCBF is modulated for a prolonged period of time following anodal and cathodal tDCS, and the relative extent of the modulation with regards to stimulation intensity. We hypothesized that the long-term mechanisms of neuroplasticity as have been previously observed in cortical excitability studies, may also be involved in a plasticity-based regulation of cerebral blood flow. As such, anodal tDCS would be expected to increase $\mathrm{rCBF}$ while cathodal tDCS should decrease it. These polarity-dependent alterations could also be expected to modulate regional functional connectivity, as also previously observed (Polanía et al., 2012b; Stagg et al., 2013; Zheng et al., 2011). As a secondary analysis, we explored the relevance of anatomical covariates on affecting inter-individual responses. Previous studies have pointed to demographic factors, such as age and gender (Kuo et al., 2006) as well as anatomical factors, such as skull thickness (Opitz 
et al., 2015), and more recently, sensitivity of the brain to non-invasive brain stimulation, such as the TMS motor threshold sensitivity (Labruna et al., 2016) as potential confounds which may affect individual sensitivity to stimulation. In addition, a recent study found a close relationship between cortical gray matter volume and intrinsic resting-state activity (Qing and Gong, 2016). As such, the effects of these factors on the physiological inter-individual responses were investigated. 


\subsection{Materials and Methods}

\subsection{Subjects}

32 healthy and non-smoking participants were recruited for the study (16 males, mean age $25.0+/-4.4 \mathrm{y}$ ). All subjects were right-handed as assessed by the Edinburg Handedness Inventory (Oldfield 1971). The study was approved by the Medical Ethics Committee of the University of Göttingen. Prior to taking part, each participant provided written informed consent and was screened by a medical professional to verify no history of neurological disease, active medication, metal implants and pregnancy. 28 of the 32 participants were naïve to receiving tDCS. Subjects were randomly divided into experimental groups of polarity (anodal and cathodal) and were blinded to both polarity and intensity of the stimulation throughout the course of the study. Initially, two participants were unable to complete the five required sessions, so two additional subjects were recruited as replacements. One individual was removed from the final analysis due to excessive head and body movement in at least two sessions, which also resulted in mislabeling of perfusion and physiologically misleading results. As such, data were analyzed from an effective sample size of 29.

\subsection{Stimulation of the motor cortex}

Online DC-stimulation of the left motor cortex was performed using an MRcompatible constant-current battery powered stimulator (neuroConn, Ilmenau, Germany). The location of the target electrode on the scalp was determined individually for each subject by using TMS-induced motor evoked potentials (MEPs) of the right hand anterior digiti minimi muscle (ADM) to locate the hand representation area over the scalp. The target electrode (35 $\mathrm{cm} 2$ ) was placed over the marked region, with a 45deg rotation towards the midline (Figure 1B). A second electrode $(100 \mathrm{~cm} 2)$, made larger in order to reduce the non-targeted current density (Nitsche et al., 2007) was placed contralaterally over the participant's right orbit. To further reduce any discomfort of the stimulation and to ensure adequate blinding, a topical anesthetic cream was pre-applied to the electrode areas on the scalp and was also layered on the bottom surface of the electrodes (Guleyupoglu et al., 2014; McFadden et al., 2011). Based on the experimental group and session conditions, anodal or cathodal tDCS at an intensity of $0.5,1.0,1.5,2.0 \mathrm{~mA}$ or sham was delivered for $15 \mathrm{~min}$, with a $10 \mathrm{sec}$ ramp at the beginning and end of stimulation. For the sham condition, a DC intensity of $1.0 \mathrm{~mA}$ was delivered for $30 \mathrm{sec}$, with a $20 \mathrm{sec}$ ramp, which has been shown to achieve effective stimulation blinding (Ambrus et al., 2012; Gandiga et al., 2006). During blocks for which no DC stimulation was delivered, electrodes were kept disconnected from the battery-driven constant-current stimulator so as to avoid any MRinduced artifacts within the electrodes.

\subsection{MR acquisition of Cerebral Blood Flow}

fMRI was conducted in a 3 Tesla Magnetom TrioTim (Siemens Healthcare, Erlangen, Germany) using a 32-channel head coil. Stimulation electrodes were fitted before subjects were placed inside the magnet bore. Initially, anatomical 
images based on a T1-weighted 3D turbo fast low angle shot (FLASH) MRI sequence at $1 \mathrm{~mm}^{3}$ isotropic resolution were recorded (repetition time (TR) $2250 \mathrm{~ms}$ echo time (TE) $3.32 \mathrm{~ms}$, inversion time $900 \mathrm{~ms}$, flip angle 9 degrees). Subsequent scans were divided in ten blocks: pre-stimulation/baseline, stimulation, and then after-effects measurements immediately as well as 15, $30,45,60,75,90,105$, and 120 minutes after stimulation. For each of the ten blocks, two measurements were obtained: a resting-state blood-oxygen-leveldependent (BOLD) measurement $(5 \min 51 \mathrm{~s})$ and a resting-state ASL measurement (5 min $8 \mathrm{~s}$ ). The order of the measurements was counterbalanced evenly between subjects to mitigate ordering effects. As we were here primarily interested in the effects of stimulation on CBF, we restricted the analysis of the present findings only to the ASL measurements; analysis of the BOLD effects as well as respective comparisons would be the scope of another study.

ASL images were acquired using a pseudo-continuous ASL (pcASL) sequence with the following parameters: TE $12 \mathrm{~ms}$, TR $3750 \mathrm{~ms}$, 24 slices, in-plane resolution, 3x3 mm, slice thickness $4 \mathrm{~mm}, 20 \%$ gap, flip angle 90, FOV 192 $\mathrm{mm}$, labelling time $1484 \mathrm{~ms}$, post-label delay $1 \mathrm{~s}$, RF gap 360 us, RF blocks 80 . Each ASL sequence was accompanied by a background-suppressed proton density (PD) reference image using the same parameters, but without ASL labeling, which was used for functional registration and CBF calibration (see preprocessing, section 2.5).

\subsection{Experimental Procedure}

Figure 1 provides an overview of the experimental procedure. Each subject underwent five experimental sessions (sham, 0.5, 1.0, 1.5 and $2.0 \mathrm{~mA}$ stimulation) in a pseudo-randomized order (uniform distribution over the group, matrix generated using MATLAB), where each session was separated by at least 7 days to avoid carry-over effects. After subjects were situated comfortably inside the scanner, an initial T1 anatomical scan was acquired, followed by the first of ten repeated scanning blocks, each of which consisted of resting-state BOLD and ASL sequences as described previously. The first block (baseline) was followed by the stimulation block, during which the two fMRI measurements were repeated while anodal or cathodal tDCS was delivered for $15 \mathrm{~min}$, as previously described. During the BOLD scan, participants were asked to fixate on a projected cross, and during the ASL scan, participants were asked to keep their eyes closed, "think about nothing in particular", but remain awake. Instructing participants to keep the eyes open during only the BOLD sequence of each block was decided in order to reduce visual fatigue, and avoid sleepiness. At the end of each block ( 13 min), participants were asked to rate their tiredness/arousal level on a visual analog scale using a remote controller, where the lowest value ' 0 ' denoted "not tired at all" and the highest value ' 10 ' indicated 'extremely tired'.

\subsection{Image preprocessing}

Functional image preprocessing steps were carried out using FSL5 (http://fmrib.ox.ac.uk/fsl) and proceeded as follows: boundary based segmentation and registration of the reference $\mathrm{PD}$ volume to the high 
resolution anatomical scan (Greve and Fischl, 2009), removal of the first four pcASL volumes to allow for magnetization equilibrium, and motion correction and extraction of the six head-movement parameters using MCFLIRT (Jenkinson et al., 2002). Perfusion-weighted images were obtained by pair-wise subtraction of tag and control volumes, followed by inversion of a basic onecompartment kinetic model describing blood transit based on labeling and post-label delay times (model parameters based on the consensus by Alsop et al., 2015), and further calibrated into absolute CBF values using the separately acquired PD image (Chappell et al., 2009). Image volumes were spatially normalized in a two-step procedure: coregistration to the subject's high resolution T1-weighted anatomical image and then realignment to the Montreal Neurological Institute (MNI) standard brain image by means of FSL's linear registration tool, FLIRT (Jenkinson et al., 2002). Lastly, images were spatially smoothed using an $8 \mathrm{~mm}$ full-width at half-maximum (FWHM) Gaussian kernel.

\subsection{ROI analysis}

Quantitative assessment of the local and regional effects of tDCS was conducted comparing the perfusion time courses between four regions of interest (ROIs): 1) the region of the target electrode, the left M1, which was defined as the gray matter areas contained in the left precentral gyrus, limited rostrally by the precentral sulcus and caudally by the Rolandic sulcus (Tzourio-Mazoyer et al., 2002); 2) the contralateral right M1 to assess possible interhemispheric/transcallosal effects, and was similarly identified as voxels of the right precentral gyrus; 3 ) the region of the reference electrode, the right frontal orbit, which consisted of the orbital regions of the superior frontal gyri, inferior to the anterior commissure/posterior commissure plane; and 4) a control region for the purpose of delineating local effects from global effects, and selected to be sufficiently remote from and of approximately the same volume as the target ROI (Stagg et al., 2009; Zheng et al., 2011). As such, this ROI was defined as the gray matter voxels contained in the right superior temporal gyrus (Tzourio-Mazoyer et al., 2002; Zheng et al., 2011).

For each individual session, the overall mean perfusion time course of the voxels in each ROI from the 4-dimensional volume was extracted using FSL functions and averaged over the time-series, resulting in 10 mean perfusion values per session. For each session, perfusion metrics were normalized to the pre-intervention baseline to obtain values representing the subject- and session-specific relative change in perfusion in the following manner:

(eq. 1)

$$
\Delta r C B F=\frac{r C B F_{t}-r C B F_{\text {Baseline }}}{r C B F_{\text {Baseline }}}
$$

Thus, positive values represented a post-intervention increase in perfusion whereas negative values represented a decrease.

Inspection of normality by means of Kolmogrov-Smirnov tests did not indicate any major outliers or deviations from normality at the group level; thus, standard parametric procedures were followed. Time and intensitydependent effects in each ROI were assessed by means of four separate 
repeated-measure ANOVAs (5 levels for factor intensity x 9 levels for factor time). Violations of non-sphericity, indicated by Mauchly's Test, were adjusted using the Greenhouse-Geisser correction when necessary. An additional, a priori assessment was conducted to delineate the focal extent of tDCS-induced changes in rCBF between 1) the target and reference electrodes; 2) the targeted region and its transcallosal region, the right M1; and 3) the target region and an outlying control region of approximate size. As such, the rCBF differences were grand-averaged over the time-course and comparisons between the respective ROIs were conducted with paired $t$-tests (two-tailed, corrected for multiple comparisons using Bonferroni's adjustment, $\mathrm{p}<0.0025$ ). In the case of a significant main effect of INTENSITY, we conducted post-hoc comparisons (two-tailed Student's paired $t$-tests, $\mathrm{p}<0.05$ uncorrected) between the respective tDCS intensity and sham. For assessing the temporal nature of the intervention's after-effects, we conducted two-tailed one-sample $t$-tests to determine whether the time-normalized response significantly differed from mean "0" (baseline) $(\mathrm{p}<0.05$, uncorrected).

\subsection{Voxelwise analysis}

As previous imaging studies have shown the ability of tDCS to induce widespread effects in rCBF across the cortex (Lang et al., 2005; Stagg et al., 2013), we also explored the remote effects of the stimulation intensities at the voxel level. Here, a whole-brain, voxel-wise analysis was conducted within the framework of a multi-level general linear model, using SPM12 (Wellcome Trust Center for Neuroimaging, UK). First, for each session, the subject's 4dimensional perfusion volume (see preprocessing) for each scanning block was entered into a first-level fixed effects model to obtain nine contrasts representing the within-session difference between the baseline (pre-tDCS) and post-stimulation perfusion (i.e., each map representing the stimulationinduced after-effects of the individual at the particular timed measurement point). These nine contrasts for each of the five sessions (for a total of 45 contrasts per subject) were entered into a second-level random effects analysis, where a 5x9 ANOVA design matrix was implemented to model the main factors of INTENSITY and TIME, as well as the INTENSITY $\mathrm{x}$ TIME interaction. SPM's flexible factorial model thus allowed us to extract $F$ contrasts for the two main effects and the interaction, as well as $t$-contrasts, which assessed the overall effect of an active-tDCS intensity $(0.5-2.0 \mathrm{~mA})$ against sham tDCS (two-tailed testing). As further exploratory analyses, we investigated the spatio-temporal dynamics of the rCBF after-effects as a function of the differential effect between each active tDCS intensity and sham. Here, the rCBF baseline-difference maps for each subject and session in the first-level output were entered into a second-level paired $t$-test analysis, which compared the mean effects between an active tDCS intensity and sham over 30 min intervals. Inference testing proceeded after contrast images were corrected for multiple comparisons by means of SPM's Gaussian Random Field correction, based on an adjustment for the family-wise error (FWE) (cluster alpha $\mathrm{p}<0.05$, voxelwise alpha $\mathrm{p}<0.01$ ).

\subsection{Functional Connectivity}


Intensity and polarity-dependent tDCS induced modulation of cortico-cortical connectivity was assessed by means of a seed-based analysis. First, in order to mitigate spurious correlations arising from head motion as well as cardiac and respiratory rhythms, we regressed each perfusion volume against the six headmotion parameters (see preprocessing), the average time-course of the white matter, calculated as the mean time-series extracted from the white matter tissue segmentation, and with the average time-course of the CSF signal, calculated as the mean time-series extracted from the lateral ventricles. Second, the time-series from the tDCS-targeted cortical region (left precentral gyrus; see ROI analysis) were extracted and correlation maps against the remaining cortical and subcortical regions (gray matter regions defined in the AAL atlas) were computed using standard procedures. Correlation maps were z-transformed in order to improve normality. To reduce the risk of false positives while maintaining adequate sensitivity, a two-stage procedure was followed to control for type I error. In the first stage, the first-level contrast maps of post-intervention connectivity differences were computed as previously described (i.e., the subject/session-specific connectivity difference at Time $x=$ [Time $\mathrm{x}$ - Time Baseline]/[Time Baseline], where $x$ includes the nine time measurements in the session following tDCS). The baseline normalized correlation coefficients were then submitted to a group-level repeatedmeasures ANOVA to assess, at each region, the main effects of STIMULATION, TIME, and STIMULATION x TIME interaction. Regions where the ANOVA indicated a significant main effect were then submitted to a second stage $t$-test, to determine if the respective intensity-wise differences between active tDCS intensities and sham tDCS was significant (two-tailed paired $t$-test, $\mathrm{p}<0.05$, corrected by Bonferroni-Holm). Effect sizes between active and sham tDCS in these identified regions are presented as Cohen's $d$, and were calculated based on the standard procedure for repeated measure designs (Dunlap et al., 1996).

We hypothesized a priori that tDCS delivered to the targeted cortical area of the left M1 would modulate functional connectivity in a polarity dependent manner; however, our findings pointed to a partially non-polarity-specific increase in connectivity for cathodal tDCS. To assess if these effects might have been due to an observed cathodal-tDCS induced increase in $\mathrm{rCBF}$ in the frontal regions (see section 3.2 and Figure 6D), we conducted an additional seed connectivity analysis, this time using the cortical region below the reference electrode as the seed-the right superior orbit. Analysis was thus repeated in the same steps as above, and for both sample groups of anodal and cathodal tDCS. For these exploratory comparisons, significance was assessed at an alpha $\mathrm{p}<0.01$.

\subsection{Inter-individual variability}

As additional analyses, we assessed the inter-subject variability of the tDCS after-effects, which remains an important issue. We identified five relevant subject-specific markers for the covariate analysis. We first assessed whether an individual's average TMS stimulus intensity required to generate a $1 \mathrm{mV}$ MEP amplitude in the ADM muscle $\left(\mathrm{SI}_{1 \mathrm{mV}}\right.$, approximately $130 \%$ of the resting 
motor threshold) might have been a factor, as it was recently identified in a multi-study analysis of cortical excitability effects (Labruna et al., 2016). Additionally, we investigated whether the skull thickness under the area of the electrode may have been relevant in affecting the induced current flow (Opitz et al., 2015). Third, we investigated whether an individual's total gray matter volume (GMV) in the targeted M1 region may have led to variable effects in rCBF. Fourth, we investigated whether the age of an individual may have been functionally relevant (Datta et al., 2012; Fujiyama et al., 2014), and lastly, we investigated whether a participant's mean arterial perfusion at baseline may have influenced the extent of the intervention's after-effect. The five covariates of interest were obtained as follows. $\mathrm{SI}_{1 \mathrm{mv}}$ was obtained as for each subject as the average intensity, measured as a percentage of the maximum stimulator output (\% MSO), over the course of five sessions, and averaged together (note that this mean metric was considered reliable as the intensities did not significantly differ between sessions- $\mathrm{SD}=1.99, \mathrm{p}>0.05)$. The electrode to cortex distance (ECD) was obtained individually per subject, based on anatomical segmentations. Briefly, the, high resolution T1 image from each subject was reconstructed as a $3 \mathrm{D}$ isosurface of the scalp, which allowed for the identification of the stereotaxic center of the target electrode. The extraction of the center coordinates in each subject's native T1 anatomical space was further guided by the attached gel-based contrast markers (see Figure 1C; reconstruction implemented in MRIcron - available open source at https://www.nitrc.org/projects/mricron). The respective T1 volume was then processed using Freesurfer (freely available http://surfer.nmr.mgh.harvard.edu/) for an advanced segmentation and highresolution parcellation of prominent cortical areas, based on cytoachitechtonic landmarks (Fischl et al., 2004). The left motor cortical region/Brodmann area 4 (BA4) was extracted, and MATLAB scripts were used to calculate the Euclidean distance between the center point of the electrode and the center point of the M1 label. The third metric-gray matter volume of the left M1was also obtained from the Freesurfer-generated segmentation, which included a volume and morphometry analysis. This analysis attains greater accuracy than standard segmentation techniques due to additional surface based calculations, tessellation of the gray matter/white matter boundary, and automated topology correction (Fischl et al., 2001; Segonne et al., 2007). As we collected individual anatomical images five times for each subject, the ECD and GMV measurements were calculated separately with each session's high resolution T1 image and then averaged together. Finally, at the group level, the subject-specific metrics were first inspected for application of standard parametric analyses by means of Kolmogrov-Smirnov goodness of fit tests, and then correlated against the individual's averaged post-stimulation rCBF response extent, calculated as the grand average difference from 0-120 min post-tDCS. Thus, we obtained a correlation coefficient for each tDCS intensity to the five factors of interest. Significance was inferred from a Bonferronicorrected alpha of $\mathrm{p}<0.01$; however, as our analysis was of an exploratory nature, results were also compared to an uncorrected $\mathrm{p}<0.05$. In order to assess the effect size of these covariates within the sample at hand, the correlation coefficients were calculated with $95 \%$ confidence intervals based on a sample- 
wise bootstrap resampling procedure (1,000 Monte-Carlo simulations). All statistical calculations were conducted using the Statistics Toolbox for MATLAB (version 2015b, Mathworks Inc.) and SPSS (v22.0, IBM Corp). 


\subsection{Results}

Table 1 summarizes the descriptive statistics of the study sample at hand. At baseline, between-session differences in global rCBF did not significantly differ for either group of anodal $(\mathrm{F}=1.444, \mathrm{~d} . \mathrm{f}=4, \mathrm{p}=0.232)$ or cathodal tDCS $(\mathrm{F}=0.642$, d.f. $=4, \mathrm{p}=0.637)$. Moreover, within-subject baseline $\mathrm{rCBF}$ was found to be fairly reliable across sessions (intra-class correlation coefficient ICC $[2,1]=0.590, \mathrm{n}=30$; Cronbach's alpha $=0.878, \mathrm{n}=5$ ).

\subsection{Region-wise modulation of $r C B F$ at different current intensities}

Quantitative assessments of region-wise $\mathrm{rCBF}$ modulation at the five intensities across nine measurement time-points were assessed using ANOVAs. Comprehensive findings from these ANOVAs are presented in Table 2 and are summarized as follows.

Anodal tDCS: For the ROI containing the left primary motor cortex, the ANOVA indicated a significant main effect of intensity $(\mathrm{F}=4.687$, d.f. $=4$, $\mathrm{p}=0.003)$ and time $(\mathrm{F}=10.372$, d.f.. $=2.974, \mathrm{p}<0.001)$. Post-hoc tests indicated significantly increased perfusion with $2.0 \mathrm{~mA}$, which remained elevated relative to the baseline for nearly the entire $2 \mathrm{~h}$ period of scanning (Figure $2 \mathrm{~B}$ ). $1.0 \mathrm{~mA}$ anodal tDCS also showed significant increases in left M1 perfusion relative to sham, and had maximal effects in the range of 0-60 min poststimulation, relative to baseline (Figure $2 \mathrm{~B}$ ). In the contralateral, right $\mathrm{M} 1$, the ANOVA indicated a main effect of time only $(\mathrm{F}=12.621$, d.f. $=2.971, \mathrm{p}<0.001)$, although $1.0 \mathrm{~mA}$ and $2.0 \mathrm{~mA}$ did not appear to follow the same pattern of effects as observed in the left $\mathrm{M} 1$-whereas $1.0 \mathrm{~mA}$ induced a decrease of contralateral M1 rCBF, $2.0 \mathrm{~mA}$ resulted in a significantly prolonged enhancement of $\mathrm{rCBF}$ in this region, and $1.5 \mathrm{~mA}$ revealed respective intermediary effects (Figure $2 \mathrm{C}$ ). In the right frontal orbit, the ANOVA showed a main effect of time $(\mathrm{F}=0.049$, d.f. $=3.143, \mathrm{p}=0.045)$, however, no main effect of intensity or intensity $x$ time interaction. Finally, the control ROI located in the right superior temporal gyrus showed a main effect of time $(\mathrm{F}=16.078$, d.f. $=4.107, \mathrm{p}<0.001)$, but also no main effects of intensity or intensity $\mathrm{x}$ time interaction. It is thus interesting to note that all ROIs showed a main effect of time, which did not appear to be modulated by tDCS as assessed by follow-up post-hoc analyses; for example, in the sham condition, $\mathrm{rCBF}$ in the control region shows a decreased $\mathrm{rCBF}$ of approximately 2-3\% after the first $30 \mathrm{~min}$, and after $2 \mathrm{~h}$, a total decrease relative to baseline of approximately $6 \%$ (Figure $2 \mathrm{H}$ ). Anodal tDCS with all active intensities appeared to induce the greatest effect in the targeted ROI of the left M1 when compared with the control region (Figure 2I). $0.5 \mathrm{~mA}$ tDCS showed minimal effects; this intensity was effective when compared to the control region only (Figure 2I), and with a maximum effect size of 0.42 relative to sham $\mathrm{tDCS}$ in the left M1 (Figure 2J). 1.0 mA tDCS was effective in enhancing rCBF of the left $\mathrm{M} 1$ while, interestingly, reducing $\mathrm{rCBF}$ in the contralateral right $\mathrm{M} 1$ (Figure 2I); the left $\mathrm{M} 1$ effects of $1.0 \mathrm{~mA}$ were of size $\mathrm{d}=0.54$ during the initial period 30-60 min post-stimulation (Figure 2J). 1.5 mA tDCS also showed effectiveness in enhanced $\mathrm{rCBF}$ in the $\mathrm{M} 1$ relative to contralateral right $\mathrm{M} 1$, the contralateral right orbit, and the control region (Figure 2J); the effect size was maximum $(\mathrm{d}=0.60)$ after 60-90 min following tDCS. More profoundly, the 
effects of $2.0 \mathrm{~mA}$ were the strongest among all intensities: an increased perfusion in the contralateral right M1 was somewhat less, but not significantly different from the increased perfusion in the left M1, while the post-stimulation difference of $\mathrm{rCBF}$ in the right orbital region and the control region was significantly less (Figure 2I). The effect of $2.0 \mathrm{~mA}$ tDCS was largest in the initial time period 0-30 min following the stimulation and in the final time block ( $\mathrm{d}=0.99, \mathrm{~d}=1.07$ respectively; Figure $2 \mathrm{~J})$.

Cathodal tDCS: An ANOVA of the mean rCBF differences in the targeted left M1 ROI showed significant main effects of intensity $(F=3.821$, d.f. $=4, p=0.008)$ and time $(\mathrm{F}=3.903$, d.f. $=3.706, \mathrm{p}=0.009)$. Similar to anodal $\mathrm{tDCS}$, a main effect of time was also observed in the right $\mathrm{M} 1 \mathrm{ROI}(\mathrm{F}=5.125$, d.f. $=4.5, \mathrm{p}<0.001)$, as well as the control ROI $(\mathrm{F}=8.452$, d.f. $=3.381, \mathrm{p}<0.001)$, however not in the contralateral frontal orbit ROI. In this latter region, however, we observed an interesting intensity $x$ time interaction $(F=0.027$, d.f. $=7.771, p=0.011)$. In follow-up post-hoc tests, the targeted left M1 showed significantly decreased rCBF after 30,60 , and $120 \mathrm{~min}$ with $2.0 \mathrm{~mA}$ as compared to sham tDCS $(\mathrm{p}<0.001$, after $120 \mathrm{~min})$. Over the remaining intensities, left M1 perfusion did not appear to be significantly modulated compared to sham tDCS, however a tendency to decrease compared to baseline was observed to be linear depending on current intensity (Figure 3A). In the contralateral right $\mathrm{M} 1$, the intensity dependent pattern was generally maintained, however these differences were not statistically significant compared to sham tDCS. Interestingly, we observed that in the right frontal orbit region, higher intensities of $1.5 \mathrm{~mA}$ and $2.0 \mathrm{~mA}$ cathodal tDCS resulted in an elevated $\mathrm{rCBF}$ during the stimulation period before declining through the remainder of the session (Figure 3E-F). Finally, when comparing each intensity's overall effect, grand-averaged over the entire $2 \mathrm{~h}$ time-course in the target region against the other regions, we did not observe a spatially selective reduction of $\mathrm{rCBF}$ in the targeted left M1 (Figure 3I); with the exception of $2.0 \mathrm{~mA}$ intensity, all tested active intensities appeared to show small to moderate decreases in all selected ROIs, which were generally less than $5 \%$ compared to baseline perfusion. Furthermore, the active cathodal tDCS intensities exhibited markedly reduced magnitude of effects compared to anodal tDCS of the same intensities, with the exception of $2.0 \mathrm{~mA}$, which resulted in a $12.28 \%$ decrease after $120 \mathrm{~min}$ versus sham tDCS $(\mathrm{p}=0.026, \mathrm{~d}=1.35$; Figure $3 \mathrm{~J})$.

\subsection{Remote effects of tDCS at different intensities}

Anodal tDCS: A whole-brain analysis revealed widespread, spatially distributed effects from all active intensities of anodal tDCS. Using F-contrasts in the general linear model, we first assessed the main effects of the experimental factors of stimulation intensity, time, and time $\mathrm{x}$ intensity interaction. For the factor intensity, three significant clusters were identified (Figure 4A): the left anterior cingulate (peak: $F(4,616)=8.87, z=4.85$, cluster $\mathrm{p}=0.019$ corrected), left superior temporal gyrus (peak: $\mathrm{F}(4,616)=8.58, \mathrm{z}=4.75$, $\mathrm{p}=0.030$ corrected), and the right parietal lobe (peak: $\mathrm{F}(4,616)=10.41, \mathrm{z}=4.72$, $\mathrm{p}=0.032$ corrected). For the factor time, the $F$-contrast identified a very large number of significant clusters $(>40)$; the peak of the major activations appeared to be located bilaterally in prefrontal and frontal areas, including the 
frontal poles, as well as dorsal parts of the precuneus and cingulate cortices (Figure 4B). An intensity $\mathrm{x}$ time interaction contrast did not reveal any significant clusters. To assess intensity-wise effects, we conducted two-tailed paired $t$-tests to contrast the overall main effects of the active intensity against the overall main effect of the sham condition. The main differences are reported in Figure 4C, accompanied with estimates of the effect size and 95\% confidence intervals, and can be summarized as follows: after FWE correction for multiple comparisons, a cluster-wise analysis for $0.5 \mathrm{~mA}$ anodal tDCS did not yield any significant activations across the whole brain. At $1.0 \mathrm{~mA}$, we observed two clusters of increased activation: the first located around the left anterior cingulate gyrus (peak: $\mathrm{T}(616)=5.30, \mathrm{z}=5.23$, cluster $\mathrm{p}=0.004$ corrected) and a second located in the left Heschl's gyrus (peak: $\mathrm{T}(616)=5.05, \mathrm{z}=4.99$, cluster $\mathrm{p}=0.013$ corrected). At $1.5 \mathrm{~mA}$, a significant cluster of increased $\mathrm{rCBF}$ was only observed in the left precentral gyrus (peak: $\mathrm{T}(616)=4.38, \mathrm{z}=4.34$, $\mathrm{p}=0.048$ corrected). At $2.0 \mathrm{~mA}$, two significantly increased $\mathrm{rCBF}$ clusters were identified: the first located around the left middle frontal gyrus (peak: $\mathrm{T}(616)=5.34, \mathrm{z}=5.26, \mathrm{p}=0.002$ corrected), and the second located in the region around the left inferior frontal gyrus (peak: $\mathrm{T}(616)=4.76, \mathrm{z}=4.71, \mathrm{p}=0.011$ corrected). Contrast estimates indicated the greatest difference between an active intensity and sham was from the action of $2.0 \mathrm{~mA}$ anodal tDCS in the left middle frontal gyrus $(\mathrm{d}=3.31)$.

As an exploratory assessment, we assessed the temporal evolution of the tDCS after-effects as a function of DC intensity by means of paired $t$-tests contrasting the active intensities with the sham intensity, in intervals of $30 \mathrm{~min}$ (Figure 5). Overall, in principle alignment with the ROI analyses, the maximum effects in $\mathrm{rCBF}$ were contained in the first 30-60 min over all intensities. $0.5 \mathrm{~mA}$ anodal tDCS did not appear to produce any stable or coherent pattern of activation in the cortex, while $1.0 \mathrm{~mA}$ showed a stable and spatially constricted increase of rCBF mainly in lateral, left hemispheric regions. $1.5 \mathrm{~mA}$ did not yield qualitatively higher effects as compared to 1.0 $\mathrm{mA}$, although activated clusters close to the left precentral gyrus could be identified. Finally, $2.0 \mathrm{~mA}$ induced the greatest, and most widespread effects over both the left and right hemispheres, which further persisted over the $2 \mathrm{~h}$ of monitoring. These effects spanned over a wide landscape, including prefrontal areas, contralateral motor, and premotor areas (Figure 5).

Cathodal tDCS: F-contrasts for main effects of tDCS intensity revealed a bilateral activation in two clusters located in the left superior temporal gyrus (peak: $\mathrm{F}(4,572)=10.77, \mathrm{z}=5.47, \mathrm{p}=0.001$ corrected) and the right supramarginal gyrus (peak: $\mathrm{F}(4,572)=10.09, \mathrm{z}=5.25, \mathrm{p}=0.002$ corrected; Figure 6A). In a pattern similar to anodal tDCS, the main effect analysis for the factor time showed significant modulations throughout the prefrontal areas, with the maximum activations located bilaterally in frontal poles (Figure 6B). An intensity $\mathrm{x}$ time interaction contrast also revealed a significant cluster located in the right frontal pole (peak: $F(32,572)=2.98, \mathrm{z}=5.05, \mathrm{p}=0.009$ corrected), which appears to be in alignment with the significant interaction as also reported in the ROI analysis of this region (Figure $3 \mathrm{C}$ ). In subsequent assessments for the main intensity-wise differences to sham, paired $t$-contrasts between $0.5 \mathrm{~mA}$ cathodal tDCS and sham revealed a decreased rCBF cluster in 
the left insular cortex (peak: $\mathrm{T}(572)=4.32, \mathrm{z}=4.17, \mathrm{p}=0.023$ corrected). $1.0 \mathrm{~mA}$ showed a decreased $\mathrm{rCBF}$ cluster located in the left frontal pole region (peak: $\mathrm{T}(572)=0.88, \mathrm{z}=4.82, \mathrm{p}=0.013$ corrected), as well as a decreased CBF cluster near the left temporal gyrus (peak: $\mathrm{T}(572)=4.80, \mathrm{z}=4.74, \mathrm{p}=0.028$ corrected). Cathodal tDCS at $1.5 \mathrm{~mA}$ resulted in an increased $\mathrm{rCBF}$ cluster in the right frontal pole (peak: $\mathrm{T}(572)=4.93, \mathrm{z}=4.73, \mathrm{p}=0.034$ corrected). At $2.0 \mathrm{~mA}$, an increased $\mathrm{rCBF}$ cluster was observed in the left frontal pole region (peak: $\mathrm{T}(572)=4.45, \mathrm{z}=4.41, \mathrm{p}=0.027$ corrected) and a decreased rCBF cluster in the right supramarginal gyrus (peak: $\mathrm{T}(572)=6.10, \mathrm{z}=5.99, \mathrm{p}<0.001$ corrected).

Additional, exploratory comparisons of these intensities as a function of the time course were also evaluated by means of paired $t$-test contrasts over 30 min time intervals (Figure 7). An overall observation indicated that modulatory effects in $\mathrm{rCBF}$ were most pronounced in the time range of 30-90 min after cathodal tDCS, over all active intensities. In alignment with the ROI analyses, $1.0 \mathrm{~mA}$ and $2.0 \mathrm{~mA}$ were the only active cathodal intensities to induce lasting and stable diminution of $\mathrm{rCBF}$, which appeared to be confined around the basal portions of the midbrain and temporal regions, bilaterally. Interestingly, the higher intensities of $1.5 \mathrm{~mA}$ and $2.0 \mathrm{~mA}$ both showed increased $\mathrm{rCBF}$ over the frontal pole regions during the first $30 \mathrm{~min}$ posttDCS, in line with the previous ROI analysis of this region (see Figure $3 \mathrm{E}$ ).

\subsection{Regional alterations of functional connectivity}

Anodal tDCS: Cortico-cortical functional connectivity of the targeted tDCS region was assessed by means of a seed- based analyses, taking the left M1 ROI as the seed region and correlating its perfusion time-course with all cortical regions. An overall ANOVA was first conducted to assess overall main effects of the experimental factors, stimulation intensity, time, and interaction of intensity $\mathrm{x}$ time. Widespread changes in connectivity were observed for the factor of time as well as the interaction of the intensity and time factors (not shown). Further exploratory analysis to assess the grand-average effect of each active tDCS intensity revealed a somewhat linear intensity-dependent relationship in connectivity modulation (Figure 8A): lower intensities of 0.5 $\mathrm{mA}$ and $1.0 \mathrm{~mA}$ showed less increases in connectivity but more decreases relative to sham tDCS, while higher intensities resulted in relatively greater increases in M1 coupling to both ipsilateral and contralateral regions. All intensities appeared to result in a decreased connectivity to the occipital areas, which was especially distinct with the lower intensities (see Figure 10 for a summary of the effect sizes). Overall, connectivity of the left M1 to ipsilateral regions stayed nearly constant over the intensities, while connectivity to contralateral regions increased with higher tDCS intensities. An additional exploratory analysis to dissect the intensity $\mathrm{x}$ time interaction, and also the temporal evolution of the connectivity changes, was also conducted (Figure 8B). Overall, connectivity alterations towards the areas frontal and contralateral to the target electrode were observed, which were generally modest and local to the M1 and contralateral frontal areas.

Cathodal tDCS: The region-wise ANOVAs assessing the main effect of intensity revealed a modulation of connectivity distributed towards the contralateral frontal areas (not shown). The main effects of time again showed 
a dispersed effect, indicating a temporally evolving functional connectivity modulation. Similarly, the intensity $\mathrm{x}$ time interaction also revealed a widespread involvement of functional connectivity modulation, though mostly distributed in the contralateral right hemisphere. Our follow-up analysis to assess intensity-wise effects revealed an interesting pattern: while lower cathodal tDCS intensities of $0.5 \mathrm{~mA}$ and $1.0 \mathrm{~mA}$ resulted in largely decreased connectivity from the left M1 to contralateral frontal areas as expected, the higher intensities of $1.5 \mathrm{~mA}$ and $2.0 \mathrm{~mA}$ induced strong increase of connectivity to the same regions. The overall intra-hemispheric connectivity patterns of the active intensities, as calculated in standardized effect sizes, are summarized in Figure 10. Lower intensities generally decreased the overall left M1 functional connectivity, while the higher intensities reversed the pattern and induced functional connectivity increases to both ipsilateral and contralateral hemispheres. In assessing the intensity $\mathrm{x}$ time interaction, we observed that higher intensities appeared to result in relatively greater increases in connectivity through the early time periods following tDCS as compared to later time points.

It is thus interesting to observe that similar to $2.0 \mathrm{~mA}$ anodal tDCS, both 1.5 $\mathrm{mA}$ and $2.0 \mathrm{~mA}$ cathodal tDCS also induces enhanced functional connectivity between the left $\mathrm{M} 1$ and areas around both bilateral frontal regions (Figure 9A). Interestingly, these intensities also appeared to induce an early $\mathrm{rCBF}$ increase in the ROI of the reference electrode (overlapping the anatomical region of the right frontal orbit, see Figure 3C). As a secondary follow-up analysis, we investigated the functional connectivity of this contralateral region, hypothesizing that the early-onset $\mathrm{rCBF}$ increase of 1.5 and $2.0 \mathrm{~mA}$ cathodal tDCS (where the cathodal electrode is over the motor cortex) may also have possibly coincided with a functional connectivity increase to/from this region, and thus rendered this reference electrode functionally efficient. After repeating the connectivity analysis, based on the time series extracted from this ROI region (see ROI procedure in Methods), we observed that this region did in fact interact functionally with the left $\mathrm{M} 1$, as well as other cortical regions, when 1.5 or $2.0 \mathrm{~mA}$ cathodal tDCS was applied (Figure 11).

\subsection{Inter-individual variability}

Intensity- and polarity-dependent relationships of the five inter-individual covariate factors to rCBF after-effects induced as a result of anodal and cathodal tDCS were assessed by correlation calculations. Respective findings are reflected graphically by means of Manhattan plots (Figure 15A-B). For both anodal and cathodal tDCS, no factor accounted for a significant correlation to the mean $\mathrm{rCBF}$ effect when considered under a multiplecomparison corrected alpha error of $\mathrm{p}<0.01$. However, a less conservative alpha of $\mathrm{p}<0.05$ indicated that interestingly, the left $\mathrm{M} 1$ gray matter volume correlated negatively to $\mathrm{rCBF}$ after both anodal and cathodal sham interventions $\left(\mathrm{p}<0.05\right.$, uncorrected, $\mathrm{R}^{2}=0.32$ and 0.34 , respectively). Moreover, for anodal tDCS, this factor was moderated by a trendwise, partially linear intensity-dependent relationship, up to where $2.0 \mathrm{~mA}$ denoted a positive correlation, while for cathodal tDCS, the relationship did not evolve in any apparent systematic manner. The TMS-sensitivity based metric, SI1mV, also 
showed a linear intensity-dependent trend across both polarity conditions, where for anodal tDCS, it accounted for an effective 5.3-22.8\% of the variance over the active intensities. Electrode-to-cortex distance, baseline rCBF and age did not appear to demonstrate any clear intensity-wise relationship to $\mathrm{rCBF}$ modulation. In additional analyses to explore the relevance of $\operatorname{SI} 1 \mathrm{mV}$, we found a close coupling between a participant's SI $1 \mathrm{mV}$ and the ECD ( $\mathrm{r}=0.596$, $\mathrm{p}<0.001$ ), which corroborates with previous findings in TMS literature demonstrating a functional relationship between motor threshold and the distance of the coil to the cortex (Herbsman et al., 2009; Kozel et al., 2000; McConnell et al., 2001). Moreover, SI $1 \mathrm{mV}$ also correlated positively with gray matter volume $(\mathrm{r}=0.531, \mathrm{p}<0.001)$, which however was not found to correlate with the $\operatorname{ECD}(\mathrm{r}=0.288, \mathrm{p}=0.13)$. 


\subsection{Discussion}

4.1 Intensity-dependent action of anodal $t D C S$ on local and regional $r C B F$ For all active intensities of anodal tDCS, rCBF increased, both during and after the stimulation, in the target left primary motor cortex region. Furthermore, we observed for the first time a partially linear intensity-dependent increase in perfusion (summarized in Figure 2J). Following in general accordance with the "quasi-uniform" assumption on a linear relationship between the intensity of a uniform electric field and the amount of excitability alteration (Bikson et al., 2004), the highest intensity of $2.0 \mathrm{~mA}$ induced the greatest increase in rCBF. The increase was maximum in the first 30 min following stimulation $(11.16 \%$ increase from baseline, $\mathrm{p}<0.001)$, and remained elevated for at least up to 6090 minutes $(7.70 \%$ increase from baseline, $p=0.019$; Cohen's $d=0.71$ versus sham tDCS), which appears to align closely with previously reported TMS/cortical excitability studies of this intensity (Kuo et al., 2013). During the early time-points, the $1.0 \mathrm{~mA}$ intensity was observed to result in stronger effects as compared to $1.5 \mathrm{~mA}$, as evidenced by inducing a slightly larger increase in $\mathrm{M} 1 \mathrm{rCBF}$ between 30-60 min post-stimulation; however, for the latter intensity, the increase in perfusion was prolonged for a slightly longer duration of up to $90 \mathrm{~min}$ (an effective $\mathrm{rCBF}$ increase from baseline of $2.0 \%$, $p=0.042$, Cohen's $d=0.25$ versus sham tDCS). The temporally delayed effects of higher intensities (1.5-2.0 mA) also appear to be in accordance with findings from previous tDCS studies on motor cortical excitability (Jamil et al., 2016; Kuo et al., 2013). Finally, the lowest active intensity, $0.5 \mathrm{~mA}$, did not result in a significant change in perfusion, although a small increase was observed during stimulation periods ( $2.8 \%$ increase from baseline, $\mathrm{p}=0.13)$ before following a time course trajectory that was similar to sham tDCS.

A previous ASL study which investigated $\mathrm{rCBF}$ following short durations of tDCS at intensities ranging from 0.8 to $2.0 \mathrm{~mA}$ also reported a generally linear intensity dependent relationship with anodal tDCS (Zheng et al., 2011). The findings of this study should, however, be compared with caution, as the authors used an alternating off-on paradigm to investigate different intensities, and previous work has shown that homeostatic mechanisms are involved when stimulation is intermittently repeated (Fricke et al., 2011; Monte-Silva et al., 2013). The earliest study exploring the DC intensity parameter revealed that a minimum tDCS intensity of $0.6 \mathrm{~mA}$ for $5 \mathrm{~min}\left(35 \mathrm{~cm}^{2}\right.$ electrodes, current density $0.017 \mathrm{~mA} / \mathrm{cm} 2$ ) was required for achieving a significant change in MEPs, and that higher intensities resulted in larger and more prolonged effects in motor cortical excitability (Nitsche and Paulus, 2000). Interestingly, $0.5 \mathrm{~mA}$ anodal tDCS was also shown to robustly enhance cortical excitability in a previous study by our group (Jamil et al. 2016), thus, it appears that the lack of a strong effect in $\mathrm{rCBF}$ here may require further studies for mechanistic elucidation. One previous study which found strong cortical excitability effects with low intensities $(0.3 \mathrm{~mA})$ of DC stimulation in humans proposed that low intensities of anodal tDCS could effectively induce plasticity through activation of voltage-gated calcium channels, as these have lower voltage-dependent thresholds as compared to NMDA or AMPA receptors, which along with calcium channels, have been identified to be relevant for plasticity induction (Bastani and Jaberzadeh, 2013; Liebetanz et al., 2002; 
Nitsche et al., 2004, 2003). Here, $1.0 \mathrm{~mA}$ anodal tDCS induced an rCBF increase ranging from $3.8 \%$ during stimulation to a peak of $6.6 \%$ after $60 \mathrm{~min}$, before slowly returning towards baseline. These results are in partial accordance to a previous ASL study by Stagg et al., who observed an increase of $2-3 \%$ in perfusion during and immediately after $20 \mathrm{~min}$ of $1.0 \mathrm{~mA}$ anodal tDCS $\left(35 \mathrm{~cm}^{2}\right.$ target/reference electrodes) to the left DLPFC (Stagg et al., 2013). The stronger effects observed here in the M1 might be due to differences in receptor and neurotransmitter characteristics in this region (Arco and Mora, 2009), the structural and functional architecture of the cortex (Miller et al., 2002) and/or the different path of DC current flow into this region.

In the contralateral $\mathrm{M} 1$ region, $2.0 \mathrm{~mA}$ anodal tDCS induced an $\mathrm{rCBF}$ increase throughout the session. Due to its relatively high intensity, it could be possible that the increase in perfusion was mediated through transcallosal pathways, as we observed that functional connectivity between the two motor cortices also increased. Overall, no significant effects were observed in the contralateral right orbit region, or in the control region, which indicates a relatively selective effect. The sham condition, for both anodal and cathodal tDCS, resulted in a decreasing drift of $\mathrm{rCBF}$ over the time course. This may be the result of either higher $\mathrm{rCBF}$ at baseline (ex., due to increased anxiety levels at the start of the scan, which then taper off - van Minde et al., 2013), or from general drowsiness over the period of monitoring. Interestingly, the participants self-reported attention level remained stable throughout the course of the session (data not shown).

A voxelwise cluster analysis also revealed characteristically intensitydependent activations. Most of these activations appeared around the target electrode area, and also in regions between the target and reference electrode, similar to the findings of BOLD activations from a previous FMRI BOLD study on M1-tDCS (Stagg et al., 2009). Moreover, many of these activations overlap with regions where anodal tDCS also increased functional connectivity (see comparison of Figures 5 and 8). The widespread increase in regional and global functional connectivity following tDCS also confirms previous reports (Polanía et al., 2012b, 2011b), and lends further insight that tDCS results in the modulation of a large scale network of functionally connected regions.

\subsection{Intensity-dependent action of cathodal tDCS on local and regional $r C B F$}

For cathodal tDCS, all intensities showed a tendency towards an rCBF reduction in left $\mathrm{M} 1 \mathrm{rCBF}$ compared to baseline over the entire $2 \mathrm{~h}$ timecourse. However, with the exception of $2.0 \mathrm{~mA}$, these reductions were not significant compared to sham tDCS. In general, these findings are in line with previous reports of modest $\mathrm{rCBF}$ attenuation following cathodal tDCS (Lang et al., 2005; Stagg et al., 2013; Zheng et al., 2011). They also appear to be in agreement with previous studies on cortical excitability, which have reported a prolonged excitability reduction following cathodal tDCS at $1.0 \mathrm{~mA}$ ( $9 \mathrm{~min}$ duration - Batsikadze et al., 2013; Monte-Silva et al., 2010; Nitsche et al., 2003b, 15 min duration - Jamil et al., 2016, 18 min duration - Monte-Silva et al., 2010, 20 min duration - Batsikadze et al., 2013). A study using $0.3 \mathrm{~mA}$ 
cathodal tDCS (20 min duration) also reported an excitability reduction (Vaseghi et al., 2015). Intensities higher than $1.0 \mathrm{~mA}$ have shown either null or reversed effects. In a recent study, $1.5 \mathrm{~mA}$ and $2.0 \mathrm{~mA}$ cathodal tDCS (15 min duration) did not result in a clear excitability diminution (Jamil et al., 2016), and a previous study investigating $2.0 \mathrm{~mA}$ cathodal tDCS for 20 min duration reported a reversed effect (Batsikadze et al., 2013). The authors proposed that higher intensities of cathodal tDCS may increase post-synaptic calcium to the LTP range (Cho et al., 2001; Lisman, 2001). In contrast, here $2.0 \mathrm{~mA}$ cathodal tDCS with a 15 min duration induced a significant reduction in $\mathrm{rCBF}$ compared to sham, whereas the effect of lower intensities was much smaller in magnitude. Interpretation of these non-linear findings could consider differences in the depth of these effects, as read-outs in cortico-spinal excitability are relatively limited to select neuronal populations. Another explanation could be that cortico-spinal excitability has larger sensitivity to calcium channel functions than cerebral blood flow, which is less resistant to ion flux due to auto-regulatory mechanisms (Cipolla, 2009). Moreover, participants were not asked to perform any task in the present study, and thus, it could be possible that the lack of evoked stimuli may have attenuated the CBF response independent of excitability (Caesar et al., 2003).

Interestingly, in the contralateral right orbit, higher intensities of $1.5 \mathrm{~mA}$ and $2.0 \mathrm{~mA}$ cathodal tDCS induced a short-term rCBF increase during stimulation of up to $15 \mathrm{~min}$. This finding could likely be due to the action of the anodal current electrode over this region. Moreover, a short-lasting effect was also observed in a functional connectivity analysis at these intensities (see Figure 9). Additional evidence was also obtained from a seed-based functional connectivity analysis in which the right superior frontal gyrus was set as the seed. We observed that higher intensities induced a functional connectivity increase to this region, whereas lower intensities had no effect (Figure 11). An opposite finding for anodal tDCS was not observed. These findings suggest that effects of cathodal tDCS at intensities higher than $1.5 \mathrm{~mA}$ may also be inducing effects at the return electrode, corresponding to the anodal polarity. This finding would need to be carefully considered for future experiments, where it is usually thought that if the size of the reference electrode is enlarged relative to the target electrode, it becomes functionally inactive. Indeed, this effect might depend on stimulation intensity (Nitsche et al., 2007).

\subsection{Inter-individual variability in cortical gray matter}

As a post hoc analysis, we investigated sources of inter-individual variability in our dataset. No relationship between after-effects and TMS sensitivity was found, as reported in previous cortical excitability studies (Jamil et al., 2016; Labruna et al., 2016). However, there was a small trend whereby an individual's M1 gray matter volume tended to correlate with tDCS efficacy, and which was most pronounced in the $2.0 \mathrm{~mA}$ anodal tDCS condition. The explanation for this finding is unclear. It could be possible that greater volume of gray matter may impact the electric field conductivity causing greater effects (Miranda et al., 2013). Whether this finding holds implications for gray-matter related variances in clinical subgroups is unclear. However, recent studies have highlighted the need for constructing computational models to simulate the 
electric field (Datta et al., 2012; Woods et al., 2016), and we propose that subject-specific gray matter volume could be an important physical parameter to document. 


\subsection{Conclusion}

In the present study, we investigated the effects of anodal and cathodal tDCS on regional cereberal blood flow, both during and up to $120 \mathrm{~min}$ after stimulation. We observed that $0.5-1.5 \mathrm{~mA}$ intensities of anodal tDCS resulted in modest $\mathrm{rCBF}$ increases (3-5\%) in the targeted region of the left motor cortex, which returned to baseline after 60-75 min. $2.0 \mathrm{~mA}$ anodal tDCS resulted in the greatest increase, which lasted the entire $2 \mathrm{~h}$ duration. Whereas cathodal intensities less than $2.0 \mathrm{~mA}$ did not appear to show any strong effect, an intensity of $2.0 \mathrm{~mA}$ resulted in decreased perfusion compared to sham tDCS, which was also present up to the end of the $2 \mathrm{~h}$ monitoring. These findings indicate, for the first time, that application of weak currents to the resting state cortex not only alters cortical excitability, but also leads to prolonged changes in cortical perfusion, which can span for at least $2 \mathrm{~h}$. Whether these effects could be even greater during a motor task activity is not known, and could be an interesting further study. One previous study combining theta-burst TMS with ASL imaging found increased CBF after TBS, but not with sham, when participants were asked to tap fingers (Orosz et al., 2012). However, ASL sequences during such experiments may not be well suited, due to the relatively longer temporal sampling rate. Here, newer scanning sequences, such as dual-acquisition ASL/BOLD might be a viable option (Fernández-Seara et al., 2016). Another limitation of the study is the uncertainty regarding the arousal state of participants during the relatively long course of the scanning $(\sim 2.5 \mathrm{~h})$. Participants were instructed to keep their eyes open and fixated to a crosshair during the resting-state BOLD sequences, but not during ASL. This was decided so that participants would not feel tired or exhausted from having to keep their eyes open over the entire duration of the session. Nevertheless, to ensure active vigilance, we asked participants to respond to a questionnaire every $15 \mathrm{~min}$, which asked to rate their level of tiredness. Interestingly, it was observed that most participants did not report to feeling tired or sleepy (median arousal score after the final block was 3.5/10, where 10 equates to feeling extremely tired), which was also confirmed by an offline head motion analysis to verify if there was constant intermittent activity (not exceeding the cut-off for excessive motion). Nevertheless, simultaneous heart rate or EEG recording to monitor arousal could be a future solution. Finally, the sample size of 15 subjects per group may also be a further limitation, considering we observed relatively small to medium effect sizes. However, the present study used a longitudinal approach, by which each participant also took part in a control condition, and thus, any putative effect is detected directly from the repeated measures. Thus, our findings should provide adequate implications, irrespective of the moderate sample size.

\section{Acknowledgements}

We would like to thank Fatemeh Yavari, Gunther Helms, Carsten SchmidtSamoa, Ilona Pfahlert, Britta Perl, and Severin Heumüller for valuable support and technical assistance in the completion of this work. 


\section{Funding}

This study was supported by a research grant from the German Federal Ministry of Education and Research (BMBF) (grant 'EYE-TSS', 03IPT605E). 


\section{References}

Alsop, D.C., Detre, J.A., Golay, X., Günther, M., Hendrikse, J., HernandezGarcia, L., Lu, H., Macintosh, B.J., Parkes, L.M., Smits, M., Van Osch, M.J.P., Wang, D.J.J., Wong, E.C., Zaharchuk, G., 2015. Recommended implementation of arterial spin-labeled Perfusion mri for clinical applications: A consensus of the ISMRM Perfusion Study group and the European consortium for ASL in dementia. Magn. Reson. Med. 73, 102116. doi: $10.1002 / \mathrm{mrm} .25197$

Ambrus, G.G., Al-Moyed, H., Chaieb, L., Sarp, L., Antal, A., Paulus, W., 2012. The fade-in - Short stimulation - Fade out approach to sham tDCS Reliable at $1 \mathrm{~mA}$ for naïve and experienced subjects, but not investigators. Brain Stimul. 5, 499-504. doi:10.1016/j.brs.2011.12.001

Arco, A. Del, Mora, F., 2009. Neurotransmitters and prefrontal cortex-limbic system interactions: implications for plasticity and psychiatric disorders. J. Neural Transm. 116, 941-952. doi:10.1007/s00702-009-0243-8

Asberg, A., Holm, T., Vassbotn, T., Andreassen, A.K., Hartmann, A., 1999. Nonspecific microvascular vasodilation during iontophoresis is attenuated by application of hyperosmolar saline. Microvasc. Res. 58, 4148. doi:10.1006/mvre.1999.2153

Attwell, D., Buchan, A.M., Charpak, S., Lauritzen, M., Macvicar, B.A., Newman, E.A., 2010. Glial and neuronal control of brain blood flow. Nature 468, 232-43. doi:10.1038/nature09613

Bastani, A., Jaberzadeh, S., 2013. Differential Modulation of Corticospinal Excitability by Different Current Densities of Anodal Transcranial Direct Current Stimulation. PLoS One 8, 1-8. doi:10.1371/journal.pone.0072254

Batsikadze, G., Moliadze, V., Paulus, W., Kuo, M.-F., Nitsche, M.A., 2013. Partially non-linear stimulation intensity-dependent effects of direct current stimulation on motor cortex excitability in humans. J. Physiol. 591, 1987-2000. doi:10.1113/jphysiol.2012.249730

Berliner, M.N., 1997. Skin microcirculation during tapwater iontophoresis in humans: cathode stimulates more than anode. Microvasc. Res. 54, 74-80. doi:10.1006/mvre.1997.2025

Bikson, M., Inoue, M., Akiyama, H., Deans, J.K., Fox, J.E., Miyakawa, H., Jefferys, J.G.R., 2004. Effects of uniform extracellular DC electric fields on excitability in rat hippocampal slices in vitro. J. Physiol. 557, 175-190. doi:10.1113/jphysiol.2003.055772

Brunoni, A.R., Valiengo, L., Baccaro, A., Zanão, T. a, de Oliveira, J.F., Goulart, A., Boggio, P.S., Lotufo, P. a, Benseñor, I.M., Fregni, F., 2013. The sertraline vs. electrical current therapy for treating depression clinical study: results from a factorial, randomized, controlled trial. JAMA psychiatry 70, 383-91. doi:10.1001/2013.jamapsychiatry.32

Caesar, K., Thomsen, K., Lauritzen, M., 2003. Dissociation of spikes, synaptic activity, and activity-dependent increments in rat cerebellar blood flow by tonic synaptic inhibition. Proc. Natl. Acad. Sci. U. S. A. 100, 16000-5. doi:10.1073/pnas.2635195100

Chappell, M.A., Groves, A.R., Whitcher, B., Woolrich, M.W., 2009. Variational Bayesian Inference for a Nonlinear Forward Model. IEEE Trans. Signal Process. 57, 223-236. doi:10.1109/TSP.2008.2005752 
Chew, T., Ho, K.-A., Loo, C.K., 2015. Inter- and intra-individual variability in response to transcranial direct current stimulation (tDCS) at varying current intensities. Brain Stimul. 1-8. doi:10.1016/j.brs.2015.07.031

Cho, K., Aggleton, J.P., Brown, M.W., Bashir, Z.I., 2001. An experimental test of the role of postsynaptic calcium levels in determining synaptic strength using perirhinal cortex of rat. J. Physiol. 532, 459-466. doi:10.1111/j.1469-7793.2001.0459f.x

Cipolla, M.J., 2009. Control of Cerebral Blood Flow., in: The Cerebral Circulation. p. Chap. 5.

Cooke, S.F., Bliss, T.V.P., 2006. Plasticity in the human central nervous system. Brain 129, 1659-1673. doi:10.1093/brain/awl082

Datta, A., Truong, D., Minhas, P., Parra, L.C., Bikson, M., 2012. Interindividual variation during transcranial direct current stimulation and normalization of dose using MRI-derived computational models. Front. Psychiatry 3, 1-8. doi:10.3389/fpsyt.2012.00091

Dunlap, W.P., Jose, J.M., Vaslow, J.B., Burke, M.J., 1996. Meta-analysis of experiments with matched groups or repeated measures designs. Psychol. Methods 1, 170-177. doi:10.1037/1082-989X.1.2.170

Durand, S., Fromy, B., Bouye, P., Saumet, J.L., Abraham, P., 2002. Vasodilatation in response to repeated anodal current application in the human skin relies on aspirin-sensitive mechanisms. J. Physiol. 540, 261269. doi:10.1113/jphysiol.2001.013364

Durand, S., Fromy, B., Bouyé, P., Saumet, J.L., Abraham, P., 2002. Currentinduced vasodilation during water iontophoresis $(5 \mathrm{~min}, 0.10 \mathrm{~mA})$ is delayed from current onset and involves aspirin sensitive mechanisms. J. Vasc. Res. 39, 59-71. doi:10.1159/000048994

Fernández-Seara, M.A., Rodgers, Z.B., Englund, E.K., Wehrli, F.W., 2016. Calibrated bold fMRI with an optimized ASL-BOLD dual-acquisition sequence. Neuroimage 142, 474-482. doi:10.1016/j.neuroimage.2016.08.007

Fischl, B., Liu, A., Dale, A.M., 2001. Automated manifold surgery: constructing geometrically accurate and topologically correct models of the human cerebral cortex. IEEE Trans. Med. Imaging 20, 70-80. doi: $10.1109 / 42.906426$

Fischl, B., Van Der Kouwe, A., Destrieux, C., Halgren, E., Ségonne, F., Salat, D.H., Busa, E., Seidman, L.J., Goldstein, J., Kennedy, D., Caviness, V., Makris, N., Rosen, B., Dale, A.M., 2004. Automatically Parcellating the Human Cerebral Cortex. Cereb. Cortex 14, 11-22. doi:10.1093/cercor/bhg087

Fricke, K., Seeber, a a, Thirugnanasambandam, N., Paulus, W., Nitsche, M. a, Rothwell, J.C., 2011. Time course of the induction of homeostatic plasticity generated by repeated transcranial direct current stimulation of the human motor cortex. J. Neurophysiol. 105, 1141-1149. doi:10.1152/jn.00608.2009

Fujiyama, H., Hyde, J., Hinder, M.R., Kim, S.J., McCormack, G.H., Vickers, J.C., Summers, J.J., 2014. Delayed plastic responses to anodal tDCS in older adults. Front. Aging Neurosci. 6, 1-9. doi:10.3389/fnagi.2014.00115 
Gandiga, P.C., Hummel, F.C., Cohen, L.G., 2006. Transcranial DC stimulation (tDCS): A tool for double-blind sham-controlled clinical studies in brain stimulation. Clin. Neurophysiol. 117, 845-850.

Greve, D.N., Fischl, B., 2009. Accurate and robust brain image alignment using boundary-based registration. Neuroimage 48, 63-72. doi:10.1016/j.neuroimage.2009.06.060

Guleyupoglu, B., Febles, N., Minhas, P., Hahn, C., Bikson, M., 2014. Reduced discomfort during high-definition transcutaneous stimulation using $6 \%$ benzocaine. Front. Neuroeng. 7, 28. doi:10.3389/fneng.2014.00028

Herbsman, T., Forster, L., Molnar, C., Dougherty, R., Christie, D., Koola, J., Ramsey, D., Morgan, P.S., Bohning, D.E., George, M.S., Nahas, Z., 2009. Motor threshold in transcranial magnetic stimulation: The impact of white matter fiber orientation and skull-to-cortex distance. Hum. Brain Mapp. 30, 2044-2055. doi:10.1002/hbm.20649

Iadecola, C., 2004. Neurovascular regulation in the normal brain and in Alzheimer's disease. Nat. Rev. Neurosci. 5, 347-360. doi:10.1038/nrn1387

Jamil, A., Batsikadze, G., Kuo, H.-I., Labruna, L., Hasan, A., Paulus, W., Nitsche, M.A., 2016. Systematic evaluation of the impact of stimulation intensity on neuroplastic after-effects induced by transcranial direct current stimulation. J. Physiol. 0, 1-16. doi:10.1113/JP272738

Jenkinson, M., Bannister, P., Brady, M., Smith, S., 2002. Improved optimization for the robust and accurate linear registration and motion correction of brain images. Neuroimage 17, 825-41.

Kozel, F.A., Nahas, Z.H., DeBrux, C., Molloy, M., Lorberbaum, J.P., Bohning, D.E., Risch, S.C., George, M.S., 2000. How coil-cortex distance relates to age, motor threshold, and antidepressant response to repetitive transcranial magnetic stimulation. J. Neuropsychiatry Clin. Neurosci. 12, 376-384. doi:10.1176/jnp.12.3.376

Kuo, H.I., Bikson, M., Datta, A., Minhas, P., Paulus, W., Kuo, M.F., Nitsche, M.A., 2013. Comparing cortical plasticity induced by conventional and high-definition 4 ?? 1 ring tDCS: A neurophysiological study. Brain Stimul. 6, 644-648. doi:10.1016/j.brs.2012.09.010

Kuo, M.-F., Paulus, W., Nitsche, M.A., 2006. Sex differences in cortical neuroplasticity in humans. Neuroreport 17, 1703-1707. doi:10.1097/01.wnr.0000239955.68319.c2

Kuo, M.F., Paulus, W., Nitsche, M.A., 2014. Therapeutic effects of noninvasive brain stimulation with direct currents (tDCS) in neuropsychiatric diseases. Neuroimage 85, 948-960. doi:10.1016/j.neuroimage.2013.05.117

Labruna, L., Jamil, A., Fresnoza, S., Batsikadze, G., Kuo, M.F., Vanderschelden, B., Ivry, R.B., Nitsche, M.A., 2016. Efficacy of Anodal Transcranial Direct Current Stimulation is Related to Sensitivity to Transcranial Magnetic Stimulation. Brain Stimul. 9, 8-15. doi:10.1016/j.brs.2015.08.014

Lang, N., Siebner, H.R., Ward, N.S., Lee, L., Nitsche, M.A., Paulus, W., Rothwell, J.C., Lemon, R.N., Frackowiak, R.S., 2005. How does transcranial DC stimulation of the primary motor cortex alter regional neuronal activity in the human brain? Eur. J. Neurosci. 22, 495-504. doi:10.1111/j.1460-9568.2005.04233.x 
Lefaucheur, J.-P., Antal, A., Ayache, S.S., Benninger, D.H., Brunelin, J., Cogiamanian, F., Cotelli, M., De Ridder, D., Ferrucci, R., Langguth, B., Marangolo, P., Mylius, V., Nitsche, M.A., Padberg, F., Palm, U., Poulet, E., Priori, A., Rossi, S., Schecklmann, M., Vanneste, S., Ziemann, U., Garcia-Larrea, L., Paulus, W., 2016. Evidence-based guidelines on the therapeutic use of transcranial direct current stimulation (tDCS). Clin. Neurophysiol. 128, 56-92. doi:10.1016/j.clinph.2016.10.087

Liebetanz, D., Nitsche, M.A., Tergau, F., Paulus, W., 2002. Pharmacological approach to the mechanisms of transcranial DC-stimulation-induced after-effects of human motor cortex excitability. Brain 125, 2238-47. doi:10.1093/brain/awf238

Lisman, J.E., 2001. Three Ca2+ levels affect plasticity differently: The LTP zone, the LTD zone and no man's land. J. Physiol. 532, 285. doi:10.1111/j.1469-7793.2001.0285f.x

López-Alonso, V., Cheeran, B., Río-Rodríguez, D., Fernández-Del-Olmo, M., 2014. Inter-individual variability in response to non-invasive brain stimulation paradigms. Brain Stimul. 7, 372-80. doi:10.1016/j.brs.2014.02.004

Lüscher, C., Malenka, R.C., 2012. NMDA receptor-dependent long-term potentiation and long-term depression (LTP/LTD). Cold Spring Harb. Perspect. Biol. 4, 1-16. doi:10.1101/cshperspect.a005710

McConnell, K.A., Nahas, Z., Shastri, A., Lorberbaum, J.P., Kozel, F.A., Bohning, D.E., George, M.S., 2001. The transcranial magnetic stimulation motor threshold depends on the distance from coil to underlying cortex: A replication in healthy adults comparing two methods of assessing the distance to cortex. Biol. Psychiatry 49, 454-459. doi:10.1016/S00063223(00)01039-8

McFadden, J.L., Borckardt, J.J., George, M.S., Beam, W., 2011. Reducing procedural pain and discomfort associated with transcranial direct current stimulation. Brain Stimul. 4, 38-42. doi:10.1016/j.brs.2010.05.002

Miller, E.K., Freedman, D.J., Wallis, J.D., 2002. The prefrontal cortex: categories, concepts and cognition. Philos. Trans. R. Soc. Lond. B. Biol. Sci. 357, 1123-36. doi:10.1098/rstb.2002.1099

Miranda, P.C., Mekonnen, A., Salvador, R., Ruffini, G., 2013. The electric field in the cortex during transcranial current stimulation. Neuroimage 70 , 48-58. doi:10.1016/j.neuroimage.2012.12.034

Monte-Silva, K., Kuo, M.-F., Liebetanz, D., Paulus, W., Nitsche, M. a, 2010. Shaping the optimal repetition interval for cathodal transcranial direct current stimulation (tDCS). J. Neurophysiol. 103, 1735-1740. doi:10.1152/jn.00924.2009

Monte-Silva, K., Kuo, M.F., Hessenthaler, S., Fresnoza, S., Liebetanz, D., Paulus, W., Nitsche, M.A., 2013. Induction of late LTP-like plasticity in the human motor cortex by repeated non-invasive brain stimulation. Brain Stimul. 6, 424-432. doi:10.1016/j.brs.2012.04.011

Morris, S.J., Shore, A.C., Tooke, J.E., 1995. Responses of the skin microcirculation to acetylcholine and sodium nitroprusside in patients with NIDDM. Diabetologia 38, 1337-1344. doi:10.1007/BF00401767 
Nitsche, M.A., 2011. Beyond the target area: remote effects of non-invasive brain stimulation in humans. J. Physiol. 589, 3053-3054. doi:10.1113/jphysiol.2011.211599

Nitsche, M.A., Boggio, P.S., Fregni, F., Pascual-Leone, A., 2009. Treatment of depression with transcranial direct current stimulation (tDCS): A Review. Exp. Neurol. 219, 14-19. doi:10.1016/j.expneurol.2009.03.038

Nitsche, M.A., Cohen, L.G., Wassermann, E.M., Priori, A., Lang, N., Antal, A., Paulus, W., Hummel, F., Boggio, P.S., Fregni, F., Pascual-Leone, A., 2008. Transcranial direct current stimulation: State of the art 2008. Brain Stimul. 1, 206-223. doi:10.1016/j.brs.2008.06.004

Nitsche, M.A., Doemkes, S., Karakose, T., Antal, A., Liebetanz, D., Lang, N., Tergau, F., Paulus, W., 2007. Shaping the Effects of Transcranial Direct Current Stimulation of the Human Motor Cortex Shaping the Effects of Transcranial Direct Current Stimulation of the Human Motor Cortex. J. Neurophysiol. 97, 3109-3117. doi:10.1152/jn.01312.2006

Nitsche, M.A., Fricke, K., Henschke, U., Schlitterlau, A., Liebetanz, D., Lang, N., Henning, S., Tergau, F., Paulus, W., 2003. Pharmacological modulation of cortical excitability shifts induced by transcranial direct current stimulation in humans. J. Physiol. 553, 293-301. doi:10.1113/jphysiol.2003.049916

Nitsche, M.A., Fricke, K., Henschke, U., Schlitterlau, A., Liebetanz, D., Lang, N., Henning, S., Tergau, F., Paulus, W., 2003. Pharmacological modulation of cortical excitability shifts induced by transcranial direct current stimulation in humans. J. Physiol. 553, 293-301.

Nitsche, M.A., Grundey, J., Liebetanz, D., Lang, N., Tergau, F., Paulus, W., 2004. Catecholaminergic consolidation of motor cortical neuroplasticity in humans. Cereb. Cortex 14, 1240-1245. doi:10.1093/cercor/bhh085

Nitsche, M.A., Nitsche, M.S., Klein, C.C., Tergau, F., Rothwell, J.C., Paulus, W., 2003. Level of action of cathodal DC polarisation induced inhibition of the human motor cortex. Clin. Neurophysiol. 114, 600-604. doi:10.1016/S1388-2457(02)00412-1

Nitsche, M.A., Paulus, W., 2000. Excitability changes induced in the human motor cortex by weak transcranial direct current stimulation. J. Physiol. 527 Pt 3, 633-9. doi:PHY_1055 [pii]

Notturno, F., Marzetti, L., Pizzella, V., Uncini, A., Zappasodi, F., 2014. Local and remote effects of transcranial direct current stimulation on the electrical activity of the motor cortical network. Hum. Brain Mapp. 35, 2220-2232. doi:10.1002/hbm.22322

Opitz, A., Paulus, W., Will, A., Thielscher, A., 2015. Anatomical determinants of the electric field during transcranial direct current stimulation. Neuroimage 109, 2. doi:10.1016/j.neuroimage.2015.01.033

Orosz, A., Jann, K., Wirth, M., Wiest, R., Dierks, T., Federspiel, A., 2012. Theta burst TMS increases cerebral blood flow in the primary motor cortex during motor performance as assessed by arterial spin labeling (ASL). Neuroimage 61, 599-605. doi:10.1016/j.neuroimage.2012.03.084

Polanía, R., Nitsche, M.A., Korman, C., Batsikadze, G., Paulus, W., 2012a. The importance of timing in segregated theta phase-coupling for cognitive performance. Curr. Biol. 22, 1314-1318. doi:10.1016/j.cub.2012.05.021 
Polanía, R., Nitsche, M.A., Paulus, W., 2011a. Modulating functional connectivity patterns and topological functional organization of the human brain with transcranial direct current stimulation. Hum. Brain Mapp. 32, 1236-1249. doi:10.1002/hbm.21104

Polanía, R., Paulus, W., Antal, A., Nitsche, M.A., 2011b. Introducing graph theory to track for neuroplastic alterations in the resting human brain: A transcranial direct current stimulation study. Neuroimage 54, 2287-2296. doi:10.1016/j.neuroimage.2010.09.085

Polanía, R., Paulus, W., Nitsche, M.A., 2012b. Modulating cortico-striatal and thalamo-cortical functional connectivity with transcranial direct current stimulation. Hum. Brain Mapp. 33, 2499-2508. doi:10.1002/hbm.21380

Qing, Z., Gong, G., 2016. Size matters to function:Brain volume correlates with intrinsic brain activity across healthy individuals. Neuroimage c. doi:10.1016/j.neuroimage.2016.06.046

Roy, A., Baxter, B., He, B., 2014. High-Definition Transcranial Direct Current Stimulation Induces Both Acute and Persistent Changes in Broadband Cortical Synchronization: A Simultaneous tDCS - EEG Study 61, 19671978.

Segonne, F., Pacheco, J., Fischl, B., 2007. Geometrically Accurate TopologyCorrection of Cortical Surfaces Using Nonseparating Loops. IEEE Trans. Med. Imaging 26, 518-529. doi:10.1109/TMI.2006.887364

Shin, Y.-I., Foerster, Á., Nitsche, M.A., 2015. Transcranial direct current stimulation (tDCS) - application in neuropsychology. Neuropsychologia 69, 154-75. doi:10.1016/j.neuropsychologia.2015.02.002

Stagg, C.J., Lin, R.L., Mezue, M., Segerdahl, A., Kong, Y., Xie, J., Tracey, I., 2013. Widespread modulation of cerebral perfusion induced during and after transcranial direct current stimulation applied to the left dorsolateral prefrontal cortex. J. Neurosci. 33, 11425-31. doi:10.1523/JNEUROSCI.3887-12.2013

Stagg, C.J., Nitsche, M.A., 2011. Physiological Basis of Transcranial Direct Current Stimulation. Neurosci. 17, 37-53.

Stagg, C.J., O’Shea, J., Kincses, Z.T., Woolrich, M., Matthews, P.M., JohansenBerg, H., 2009. Modulation of movement-associated cortical activation by transcranial direct current stimulation. Eur. J. Neurosci. 30, 1412-23. doi:10.1111/j.1460-9568.2009.06937.x

Strube, W., Bunse, T., Malchow, B., Hasan, A., 2015. Efficacy and Interindividual Variability in Motor-Cortex Plasticity following Anodal tDCS and Paired-Associative Stimulation. Neural Plast. 2015, 1-10. doi: $10.1155 / 2015 / 530423$

Tzourio-Mazoyer, N., Landeau, B., Papathanassiou, D., Crivello, F., Etard, O., Delcroix, N., Mazoyer, B., Joliot, M., 2002. Automated anatomical labeling of activations in SPM using a macroscopic anatomical parcellation of the MNI MRI single-subject brain. Neuroimage 15, $273-$ 289. doi:10.1006/nimg.2001.0978

van Minde, D., Klaming, L., Weda, H., 2013. Pinpointing Moments of High Anxiety During an MRI Examination. Int. J. Behav. Med. 1-9. doi:10.1007/s12529-013-9339-5 
Vaseghi, B., Zoghi, M., Jaberzadeh, S., 2015. Differential effects of cathodal transcranial direct current stimulation of prefrontal, motor and somatosensory cortices on cortical excitability and pain perception - a double-blind randomised sham-controlled study. Eur. J. Neurosci. 42, 2426-2437. doi:10.1111/ejn.13043

Wiethoff, S., Hamada, M., Rothwell, J.C., 2014. Variability in response to transcranial direct current stimulation of the motor cortex. Brain Stimul. 7, 468-75. doi:10.1016/j.brs.2014.02.003

Woods, A.J., Antal, A., Bikson, M., Boggio, P.S., Brunoni, A.R., Celnik, P., Cohen, L.G., Fregni, F., Herrmann, C.S., Kappenman, E.S., Knotkova, H., Liebetanz, D., Miniussi, C., Miranda, P.C., Paulus, W., Priori, A., Reato, D., Stagg, C., Wenderoth, N., Nitsche, M.A., 2016. A technical guide to tDCS, and related non-invasive brain stimulation tools. Clin. Neurophysiol. 127, 1031-1048. doi:10.1016/j.clinph.2015.11.012

Zheng, X., Alsop, D.C., Schlaug, G., 2011. Effects of transcranial direct current stimulation (tDCS) on human regional cerebral blood flow. Neuroimage $58,26-33$. 


\section{Tables and Figures}

Table 1: Baseline measurements and demographic factors. The number of subjects for each experimental condition is listed, along with the gender distribution, the mean age, and baseline regional cerebral blood flow (rCBF) $( \pm \mathrm{SD}) . \mathrm{rCBF}$ was quantified from perfusion images and calibrated against a reference proton density image using a single compartment model as previously described (see methods). No factor differed significantly between session and experimental group.

\begin{tabular}{|c|c|c|c|c|c|}
\hline \multirow[t]{2}{*}{ Experiment } & \multirow{2}{*}{$\begin{array}{l}\text { Experimental } \\
\text { Session }\end{array}$} & \multicolumn{3}{|c|}{ Subjects } & \multirow{2}{*}{$\begin{array}{l}\text { Global CBF at } \\
\text { baseline ( } m L \\
/ 100 m g / m i n)\end{array}$} \\
\hline & & $\begin{array}{l}\text { Sample } \\
\text { size } n\end{array}$ & $\operatorname{Sex}(M / F)$ & Age (y) & \\
\hline \multirow{5}{*}{$\begin{array}{l}\text { Anodal } \\
\text { stimulation }\end{array}$} & Sham & 15 & $7 / 8$ & $23.5 \pm 3.4$ & $58.41 \pm 5.00$ \\
\hline & $0.5 \mathrm{~mA}$ & 15 & $7 / 8$ & $23.5 \pm 3.4$ & $56.82 \pm 3.50$ \\
\hline & $1.0 \mathrm{~mA}$ & 15 & $7 / 8$ & $23.5 \pm 3.4$ & $58.97 \pm 3.59$ \\
\hline & $1.5 \mathrm{~mA}$ & 15 & $7 / 8$ & $23.5 \pm 3.4$ & $59.38 \pm 4.05$ \\
\hline & $2.0 \mathrm{~mA}$ & 15 & $7 / 8$ & $23.5 \pm 3.4$ & $58.01 \pm 2.55$ \\
\hline \multirow{5}{*}{$\begin{array}{l}\text { Cathodal } \\
\text { stimulation }\end{array}$} & Sham & 15 & $8 / 7$ & $27.1 \pm 5.1$ & $60.82 \pm 5.98$ \\
\hline & $0.5 \mathrm{~mA}$ & 15 & $8 / 7$ & $27.1 \pm 5.1$ & $60.24 \pm 5.57$ \\
\hline & $1.0 \mathrm{~mA}$ & 15 & $8 / 7$ & $27.1 \pm 5.1$ & $59.47 \pm 5.75$ \\
\hline & $1.5 \mathrm{~mA}$ & 15 & $8 / 7$ & $27.1 \pm 5.1$ & $57.78 \pm 5.40$ \\
\hline & $2.0 \mathrm{~mA}$ & 15 & $8 / 7$ & $27.1 \pm 5.1$ & $59.18 \pm 5.37$ \\
\hline
\end{tabular}

Table 2: Analysis of the main effects of intensity and time in rCBF modulation by anodal and cathodal tDCS. First, a one-way ANOVA was calculated for inter-session differences of the average baseline regional cerebral blood flow (rCBF) across the whole brain. Second, mean rCBF values extracted from four ROIs chosen in the cortex and relevant to our tDCS montage (see methods) were analyzed by separate two-way repeated-measures ANOVAs, where we calculated the main effects of stimulation intensity and post-stimulation time on $\mathrm{rCBF}$ difference from baseline. $\left.{ }^{*}\right)$ indicates results where $\left.\mathrm{P}<0.05 ;{ }^{* *}\right)$ indicates results where $\mathrm{P}<0.001$.

\begin{tabular}{|c|c|c|c|c|c|}
\hline Experiment & Measurement & Factor & d.f. & Fvalue & P value \\
\hline \multirow[t]{8}{*}{$\begin{array}{l}\text { Anodal } \\
\text { tDCS }\end{array}$} & $\begin{array}{l}\begin{array}{l}\text { Baseline } \\
\text { (global) }\end{array} \\
\text { rCBF }\end{array}$ & Session & 4 & 1.444 & 0.232 \\
\hline & Left M1 rCBF & Intensity & 4 & 4.687 & $0.003^{\star}$ \\
\hline & Left M1 rCBF & Time & 2.974 & 10.372 & $<0.001^{\star *}$ \\
\hline & Left M1 rCBF & $\begin{array}{l}\text { Intensity } \mathrm{x} \\
\text { Time }\end{array}$ & 7.022 & 0.925 & 0.589 \\
\hline & Right M1 rCBF & Intensity & 4 & 2.402 & 0.061 \\
\hline & Right M1 rCBF & Time & 2.971 & 12.621 & $<0.001^{\star *}$ \\
\hline & Right M1 rCBF & $\begin{array}{l}\text { Intensity } \mathrm{x} \\
\text { Time }\end{array}$ & 6.681 & 1.276 & 0.272 \\
\hline & Right Frontal & Intensity & 4 & 0.837 & 0.507 \\
\hline
\end{tabular}




\begin{tabular}{|c|c|c|c|c|c|}
\hline & \multicolumn{5}{|l|}{ Orbit rCBF } \\
\hline & $\begin{array}{l}\text { Right Frontal } \\
\text { Orbit rCBF }\end{array}$ & Time & 3.143 & 0.049 & $0.045^{\star}$ \\
\hline & $\begin{array}{l}\text { Right Frontal } \\
\text { Orbit rCBF }\end{array}$ & $\begin{array}{l}\text { Intensity } \mathrm{x} \\
\text { Time }\end{array}$ & 5.939 & 0.929 & 0.478 \\
\hline & $\begin{array}{l}\text { Control region } \\
\text { Right Superior } \\
\text { Temporal } \\
\text { Gyrus rCBF }\end{array}$ & Intensity & 4 & 1.537 & 0.204 \\
\hline & $\begin{array}{l}\text { Control region } \\
\text { Right Superior } \\
\text { Temporal } \\
\text { Gyrus rCBF }\end{array}$ & Time & 4.107 & 16.078 & $<0.001^{\star *}$ \\
\hline & $\begin{array}{l}\text { Control region } \\
\text { Right Superior } \\
\text { Temporal } \\
\text { Gyrus rCBF }\end{array}$ & $\begin{array}{l}\text { Intensity } \mathrm{x} \\
\text { Time }\end{array}$ & 7.095 & 1.288 & 0.264 \\
\hline \multirow[t]{13}{*}{$\begin{array}{l}\text { Cathodal } \\
\text { tDCS }\end{array}$} & $\begin{array}{l}\begin{array}{l}\text { Baseline } \\
\text { (global) }\end{array}\end{array}$ & Session & 4 & 0.642 & 0.637 \\
\hline & Left M1 rCBF & Intensity & 4 & 3.821 & $0.008^{*}$ \\
\hline & Left M1 rCBF & Time & 3.706 & 3.903 & $0.009^{*}$ \\
\hline & Left M1 rCBF & $\begin{array}{l}\text { Intensity } \mathrm{x} \\
\text { Time }\end{array}$ & 6.137 & 0.923 & 0.592 \\
\hline & Right M1 rCBF & Intensity & 4 & 2.799 & 0.035 \\
\hline & Right M1 rCBF & Time & 4.5 & 5.125 & $0.001^{\star}$ \\
\hline & Right M1 rCBF & $\begin{array}{l}\text { Intensity } x \\
\text { Time }\end{array}$ & 6.734 & 1.404 & 0.216 \\
\hline & $\begin{array}{l}\text { Right Frontal } \\
\text { Orbit rCBF }\end{array}$ & Intensity & 4 & 0.839 & 0.507 \\
\hline & $\begin{array}{l}\text { Right Frontal } \\
\text { Orbit rCBF }\end{array}$ & Time & 1.942 & 3.366 & 0.052 \\
\hline & $\begin{array}{l}\text { Right Frontal } \\
\text { Orbit rCBF }\end{array}$ & $\begin{array}{l}\text { Intensity } \mathrm{x} \\
\text { Time }\end{array}$ & 7.771 & 0.027 & $0.011^{\star}$ \\
\hline & $\begin{array}{l}\text { Control region } \\
\text { (Right Superior } \\
\text { Temporal } \\
\text { Gyrus rCBF) }\end{array}$ & Intensity & 4 & 1.37 & 0.257 \\
\hline & $\begin{array}{l}\text { Control region } \\
\text { Right Superior } \\
\text { Temporal } \\
\text { Gyrus rCBF }\end{array}$ & Time & 3.381 & 8.452 & $<0.001^{\star *}$ \\
\hline & $\begin{array}{l}\text { Control region } \\
\text { Right Superior } \\
\text { Temporal } \\
\text { Gyrus rCBF }\end{array}$ & $\begin{array}{l}\text { Intensity } \mathrm{x} \\
\text { Time }\end{array}$ & 4.125 & 1.398 & 0.246 \\
\hline
\end{tabular}


Figure 1. Methods. (A) The study involved 30 participants who previously took part in a TMS-based cortical excitability study with identical stimulation parameters (Jamil et al. in press). One participant had to be excluded, due to gross head movement in at least two separate experimental sessions. (B) Prior to the scanning session, the motor-cortical representation of the right abductor digiti minimi muscle (ADM) was located using single-pulse TMS. (C) The respective position on the scalp was used to place a $35 \mathrm{~cm} 2$ target electrode, rotated $45^{\circ}$ to the midline, and with the cable exiting from the right posterior edge. A larger $100 \mathrm{~cm} 2$ reference electrode was positioned over the contralateral right orbit, with the cable exiting from the right edge. The target electrode was additionally fitted with gel capsules on the four corners, which allowed for a clear localization of the electrode in resulting images. (D) Scanning sessions started with acquisition of a high resolution, T1-weighted FLASH anatomical scan, followed by the first block of two resting state scans, consisting of either BOLD (6 $\mathrm{min}$ ) or ASL (5 min) acquisitions (note that the ordering was counter-balanced across subjects). This block was repeated an additional nine times, beginning with the stimulation block, where tDCS was delivered for $15 \mathrm{~min}$ using either sham, $0.5,1.0,1.5$, or $2.0 \mathrm{~mA}$ anodal or cathodal stimulation. Subsequent measurements took place every $15 \mathrm{~min}$ following the stimulation, for up to $120 \mathrm{~min}$. (E) Regions-of-interest (ROIs) were defined according to the AAL anatomical region underneath the target electrode (left pre-central gyrus), its contralateral, functionally homotopic region, the right pre-central gyrus, as well as the area under the reference electrode (right frontal orbit) and a control region, the right superior temporal gyrus.
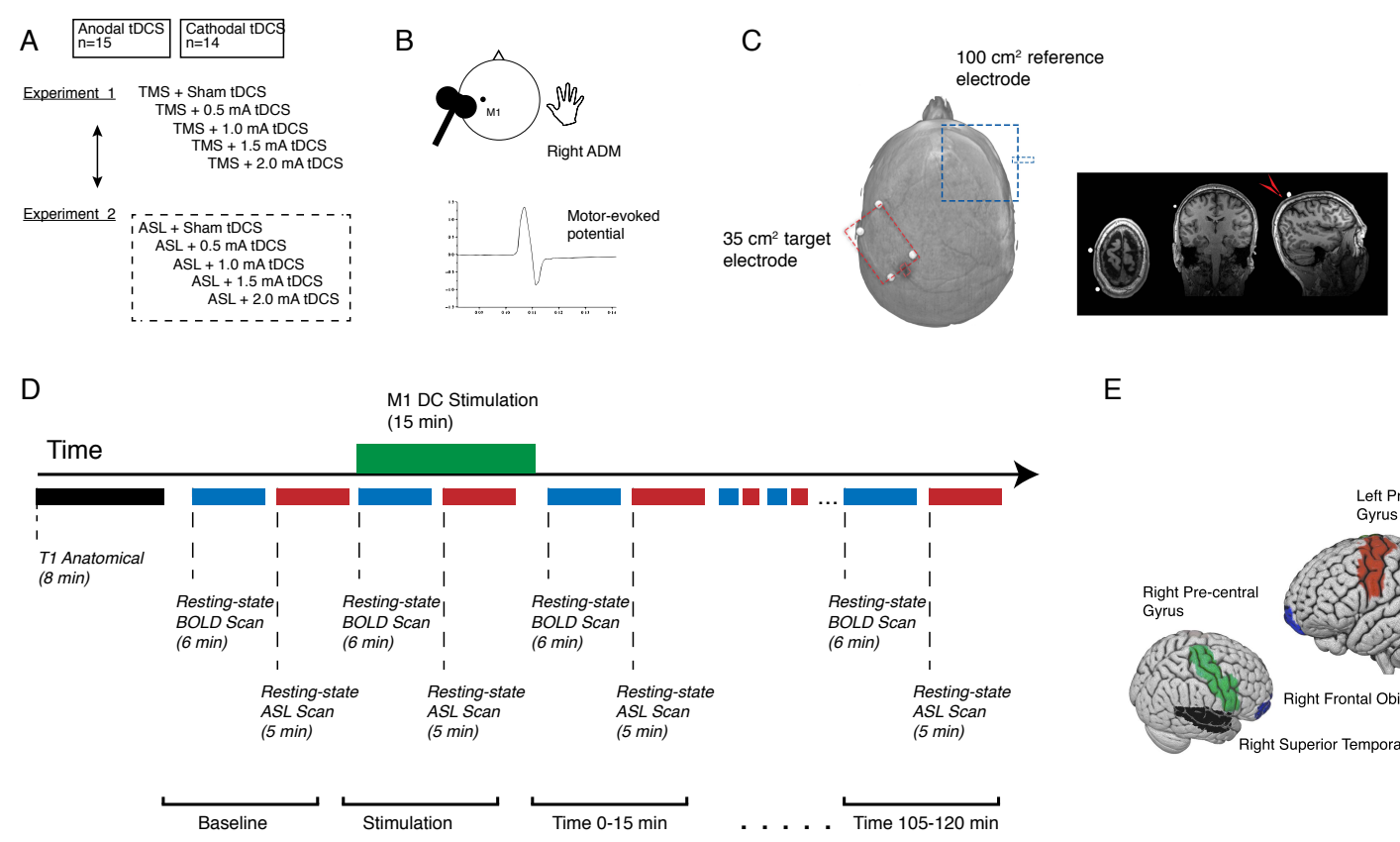

E

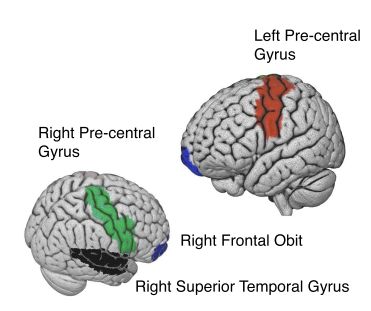


Figure 2 Perfusion time-courses within selected ROIs following anodal tDCS. ROI regions were defined according to the AAL atlas, and respective perfusion time-courses were averaged and normalized to the pre-tDCS baseline. Filled symbols indicate a significant difference $(\mathrm{p}<0.05)$ between the respective active tDCS and sham condition. Dagger symbols indicate significant differences between the time-point and the pre-tDCS baseline (single dagger when $\mathrm{p}<0.05$, double dagger when $\mathrm{p}<0.001$ ). A) In the left $\mathrm{M} 1$, which was the location of the target electrode, $2.0 \mathrm{~mA}$ resulted in significantly elevated $\mathrm{rCBF}$, compared to sham as well as baseline (B), which lasted the entire $2 \mathrm{~h}$ session and peaked between 30-60 min after tDCS. Other intensities showed no significant effects, however there was a tendency towards a linear increase in effects depending on the intensity. C) In the right M1, $2.0 \mathrm{~mA}$ increased $\mathrm{rCBF}$ initially in the first $30 \mathrm{~min}$, before it returned to baseline (D). $1.0 \mathrm{~mA}$ tended to show a decrease in $\mathrm{rCBF}$ in this region, whereas other intensities had no effect. E) In the contralateral right frontal orbit, the location of the reference electrode, no effect for any active intensity was observed when compared to sham, or with baseline (F). G) In the right superior temporal region, a distant control region from the electrodes, and of a comparable size to the M1, there was no effect on $\mathrm{rCBF}$ from any intensity relative to sham, or to baseline (H). I) A summary of the effect of different intensities (grandaveraged over the first $60 \mathrm{~min}$ ) on each ROI is presented, and shows the greatest effect of tDCS in the target left M1 region. J) Effect sizes for the differences between sham and real tDCS over different intensities on left M1 $\mathrm{rCBF}$. A linear intensity-dependence of tDCS on rCBF is observed. 
A Left M1

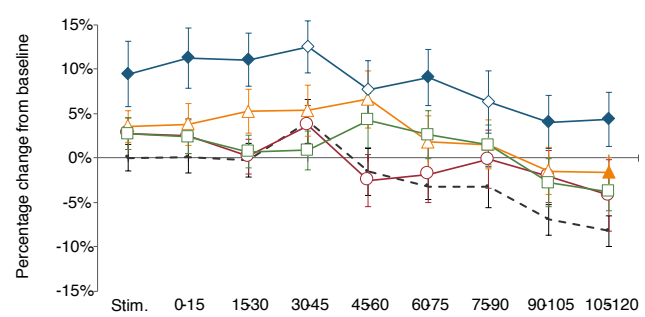

Time

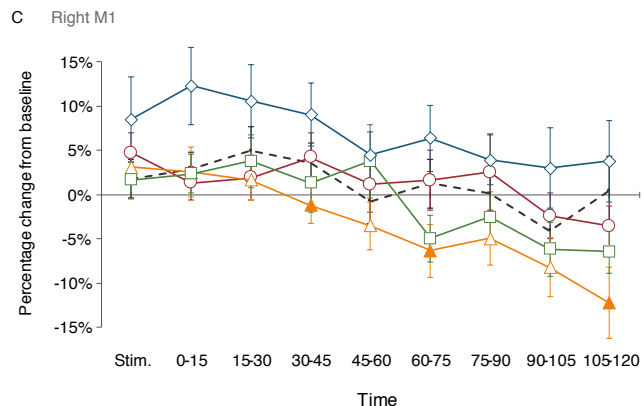

E Right Frontal Orbit

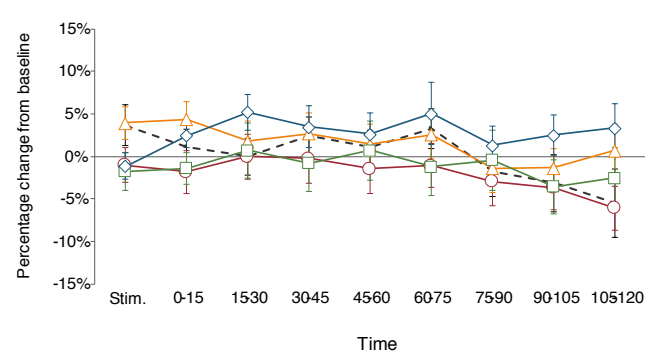

G Right Superior Temporal Gyrus

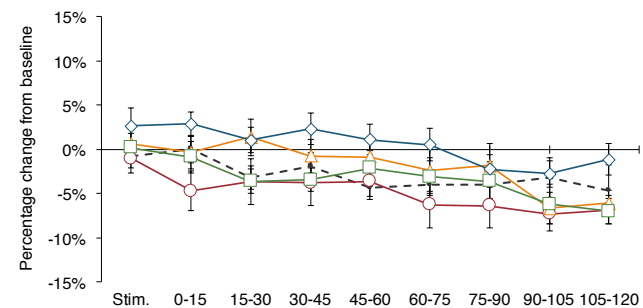

Time

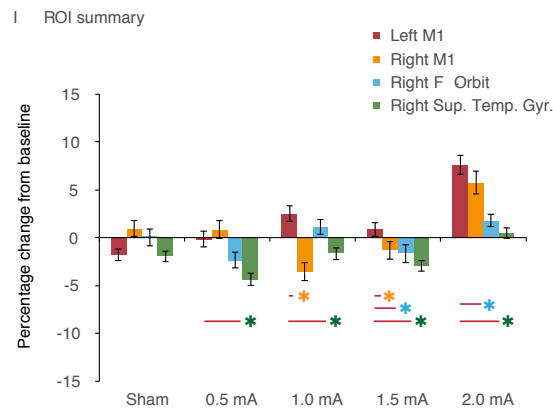

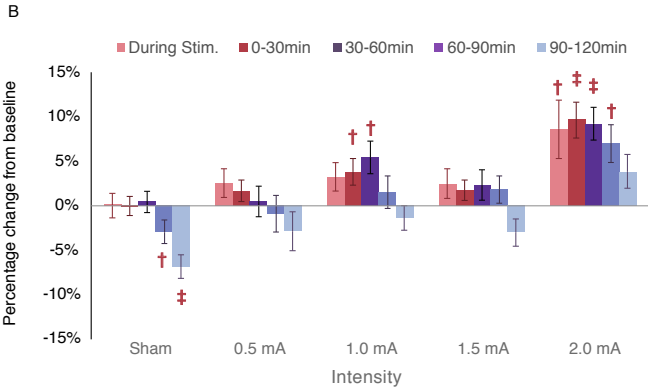

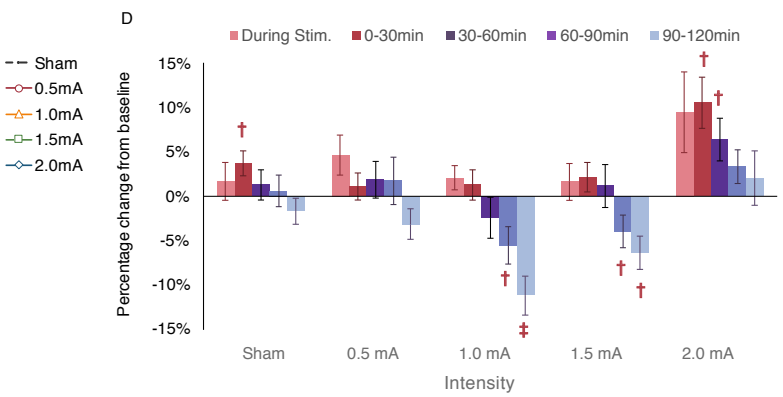

F

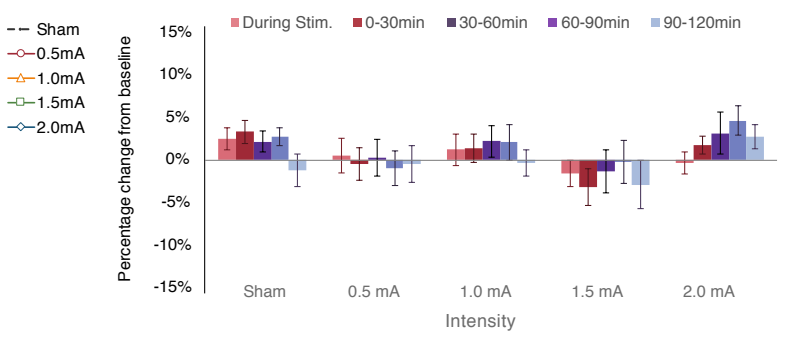

H
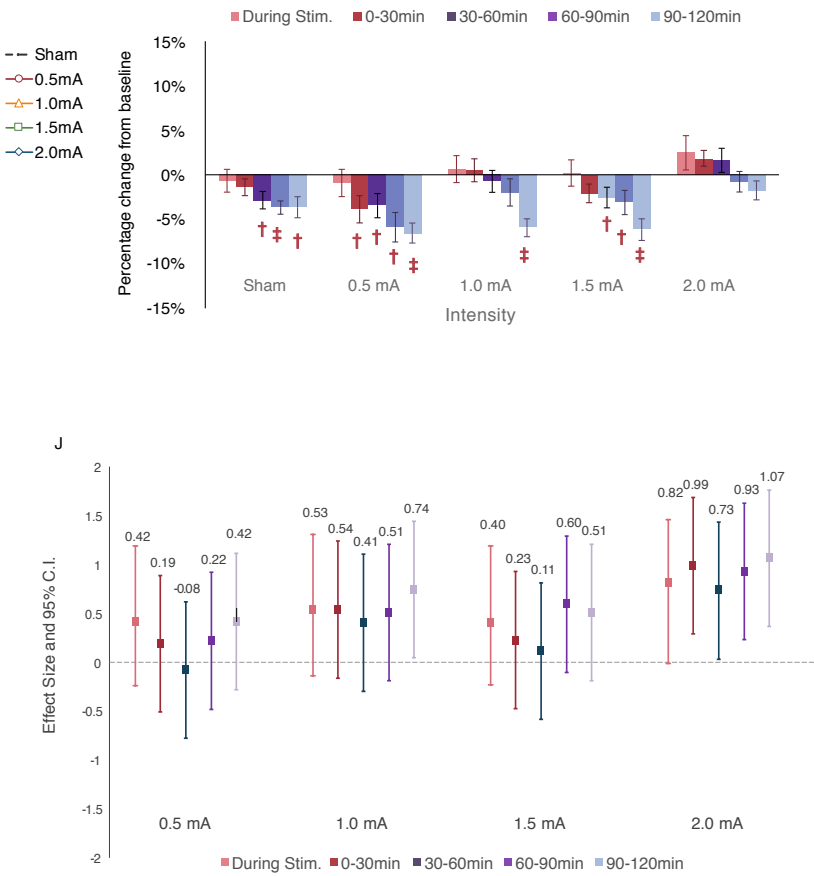
Figure 3: Cathodal tDCS - Perfusion time-courses within selected ROIs following cathodal tDCS. ROI regions were defined according to the AAL atlas, and respective perfusion time-courses were averaged and normalized to the pre-tDCS baseline. Filled symbols indicate a significant difference $(\mathrm{p}<0.05)$ between the respective active tDCS and sham condition. Dagger symbols indicate a significant difference between the time-point and the pre-tDCS baseline (single dagger when $\mathrm{p}<0.05$, double dagger when $\mathrm{p}<0.001$ ). A) In the left M1, the location of the target electrode, $2.0 \mathrm{~mA}$ resulted in significantly decreased $\mathrm{rCBF}$, compared to sham as well as baseline (B), which lasted the entire $2 \mathrm{~h}$ session. Other intensities showed no significant effects. C) In the right $\mathrm{M} 1$, a small increase in $\mathrm{rCBF}$ relative to sham was observed during stimulation for intensities of 0.5 and $1.5 \mathrm{~mA}$, however compared to pre-tDCS baseline, $2.0 \mathrm{~mA}$ induced a significant decrease beginning immediately after stimulation (D). E) In the contralateral right frontal orbit, the location of the reference electrode, an initial increase in $\mathrm{rCBF}$ was observed for higher intensities of 1.5 and $2.0 \mathrm{~mA}$ compared to sham as well as baseline (F). G) In the right superior temporal region, the control region, there was no effect on rCBF from any intensity relative to sham. All intensities, including sham, showed a reduction in $\mathrm{rCBF}$ compared to baseline over the entire time course (H). I) A summary of the effects of different intensities (grand-averaged over the first $60 \mathrm{~min}$ ) on each ROI is presented, and indicates the selective effect of $2.0 \mathrm{~mA}$ on $\mathrm{rCBF}$ modulation in the left $\mathrm{M} 1$ whereas 0.5 and $1.0 \mathrm{~mA}$ induced the greatest decrease in $\mathrm{rCBF}$ in a control region distant from the electrode. J) Effect sizes for the differences between sham and real tDCS over different intensities on left M1 rCBF. A linear intensity-dependence of tDCS on rCBF is again observed, whereby reduction in $\mathrm{rCBF}$ is more pronounced with increasing intensities. 
A Left M1

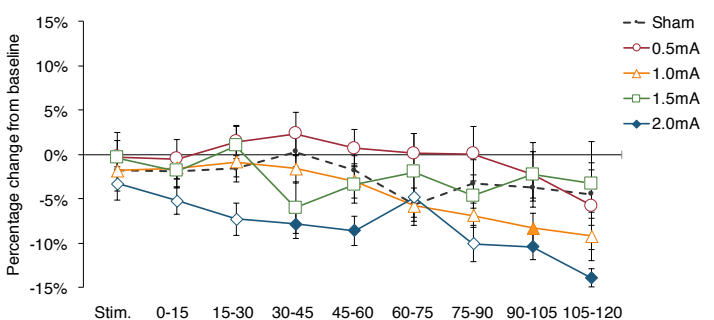

Time

C Right M1

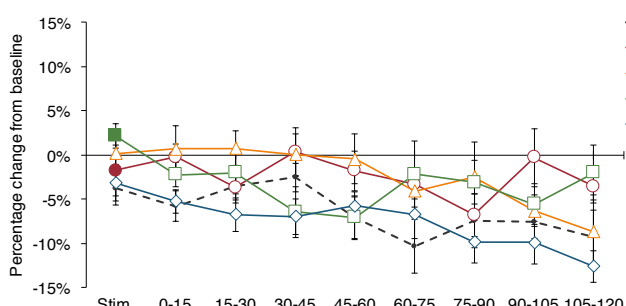

Time

E Right Frontal Orbit

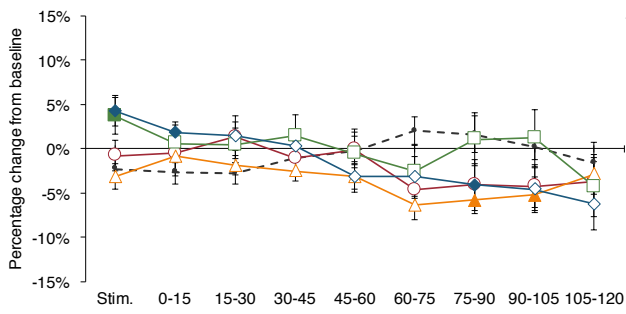

Time

G Right Superior Temporal Gyrus

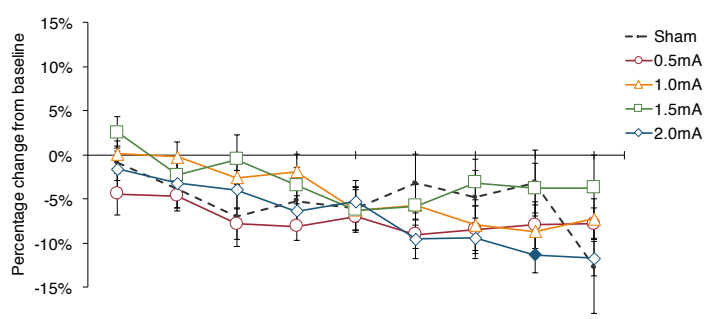

Stim. $\quad$ 0-15 $\quad 15-30 \quad 30-45 \quad 45-60 \quad 60-75 \quad 75-90 \quad 90-105 \quad 105-120$$$
\text { Time }
$$

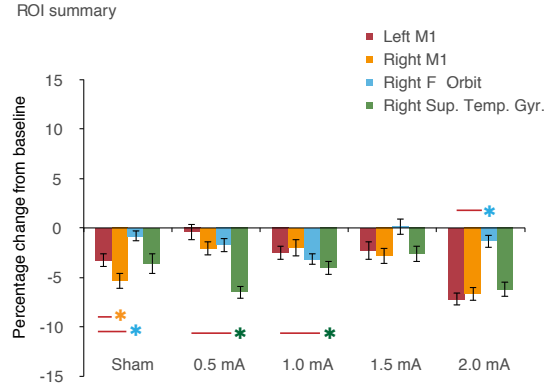

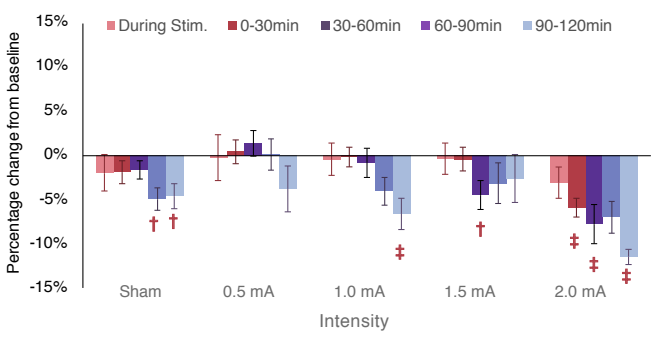
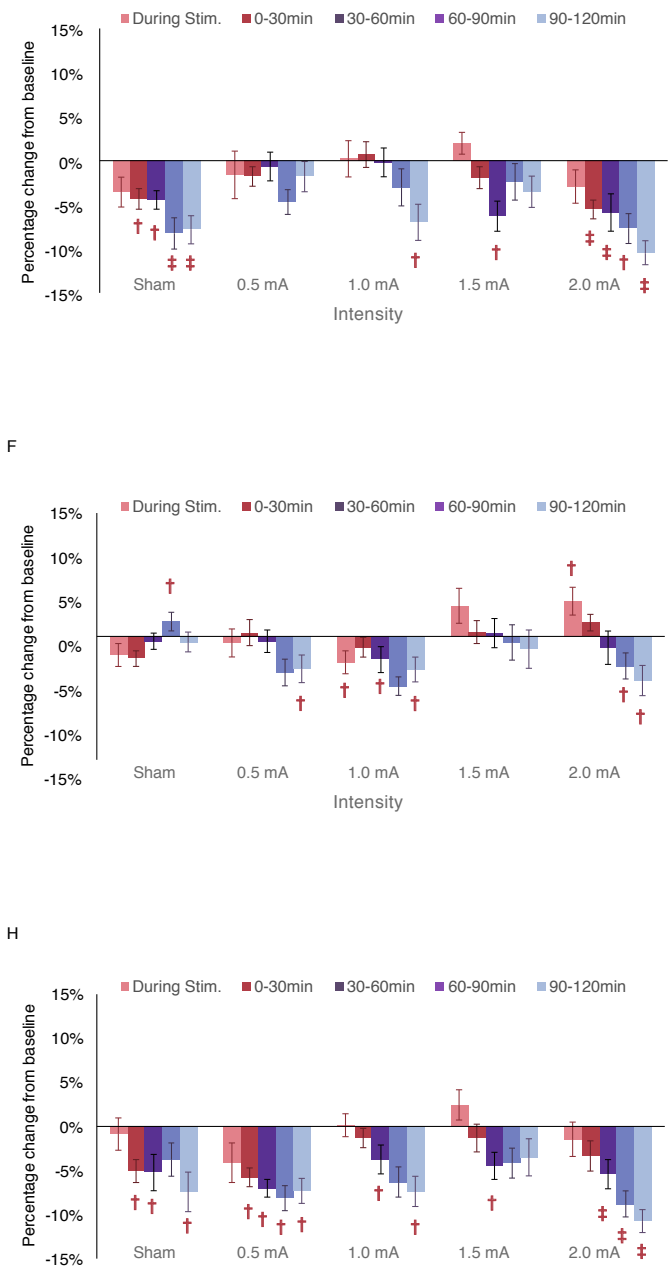

Intensity

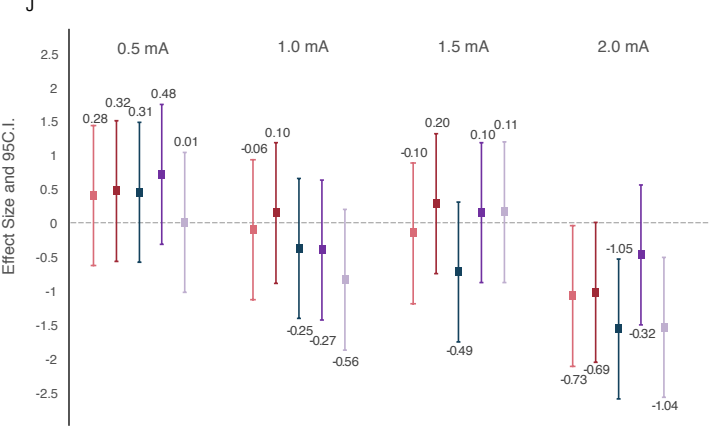

During Stim. $=0-30 \mathrm{~min}=30-60 \mathrm{~min}=60-90 \mathrm{~min}=90-120 \mathrm{~min}$ 
Figure 4. Anodal tDCS-induced modulation of rCBF activity. For contrasts of main effects, red color indicates lower critical values and yellow indicates higher critical values. For paired contrasts, blue colors indicate a negative difference and red colors indicate a positive difference. A) A main effect of stimulation shows clusters of activation in the left anterior cingulate gyrus, left superior temporal gyrus, and in deep layers of the right parietal lobe. B) Effect of time on $\mathrm{rCBF}$, collapsed over all stimulation intensities. Activations generally appeared widespread, but also overlapping with areas linked to the default mode network. C) Paired contrasts between active and sham tDCS intensities, collapsed over time, along with corresponding effect sizes. No effect observed for $0.5 \mathrm{~mA}$, whereas higher intensities induced activations mostly in the left hemisphere, and in areas around the precentral and frontal gyri. 
A

Main Effect of Stimulation Intensity

Cluster 1: Left Anterior Cingulate Gyrus
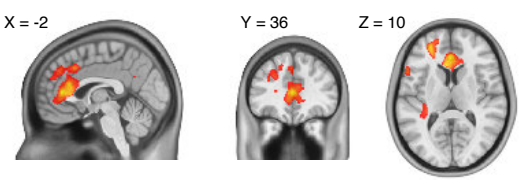

Cluster 2: Left Superior Temporal Gyrus

$\mathrm{X}=-44$

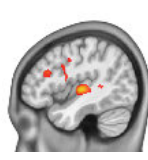

$\mathrm{Y}=-16$

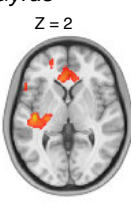

Cluster 3: Right Parietal Lobe

$\mathrm{X}=32$
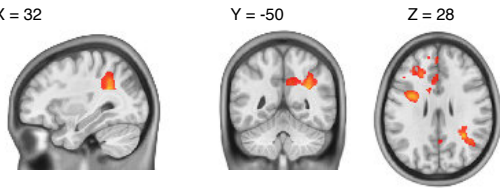

B

Main Effect of Time
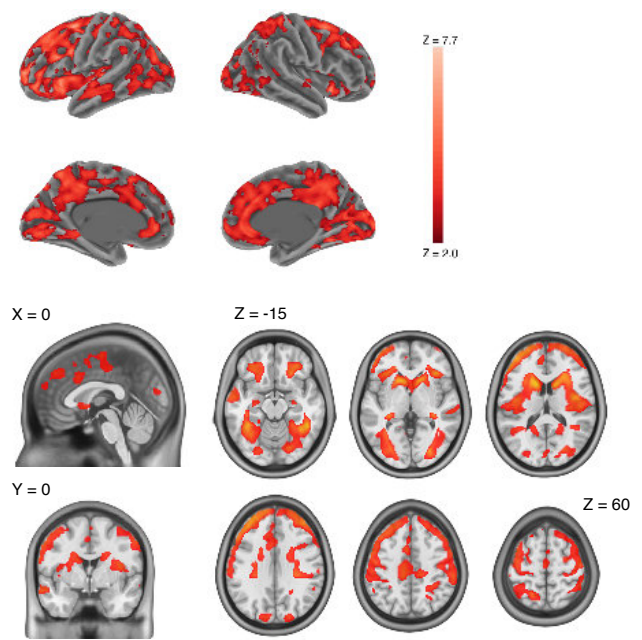

C Main effect ${ }_{\text {Active tDCS }}$ - Main effect Sham tDCs

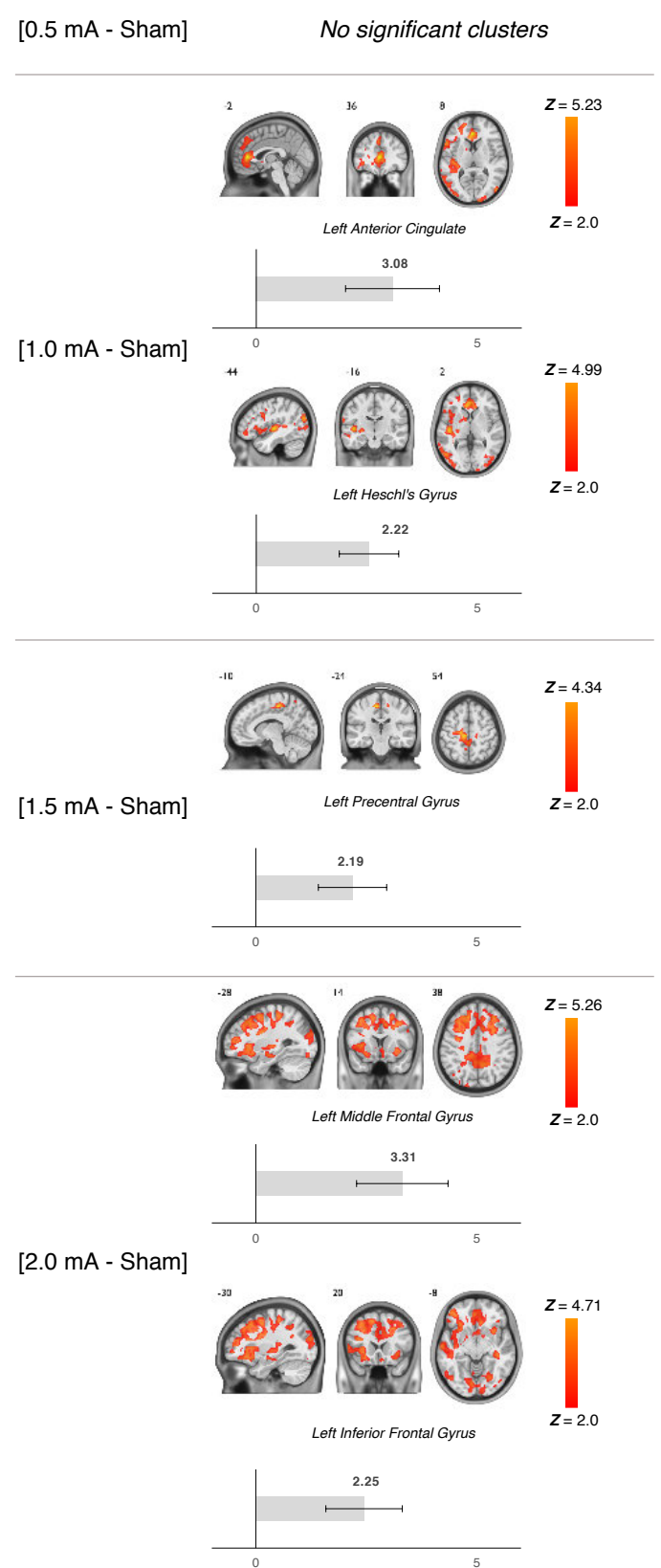


Figure 5. Exploration of time- and intensity-dependent alterations in rCBF following anodal tDCS. Perfusion maps were binned into 30 min intervals, then normalized to baseline. Paired contrasts were conducted against sham tDCS. Red colors indicate a positive difference while blue colors indicate a negative difference. Maximum effects were contained in the first 60 min over all intensities. Relative to other intensities, $0.5 \mathrm{~mA}$ showed smaller dispersed activations while $1.0,1.5$ and $2.0 \mathrm{~mA}$ resulted in widespread activations, mainly in the left hemisphere. $2.0 \mathrm{~mA}$ noticably induced the largest activation, lasting over the entire $2 \mathrm{~h}$ session. 


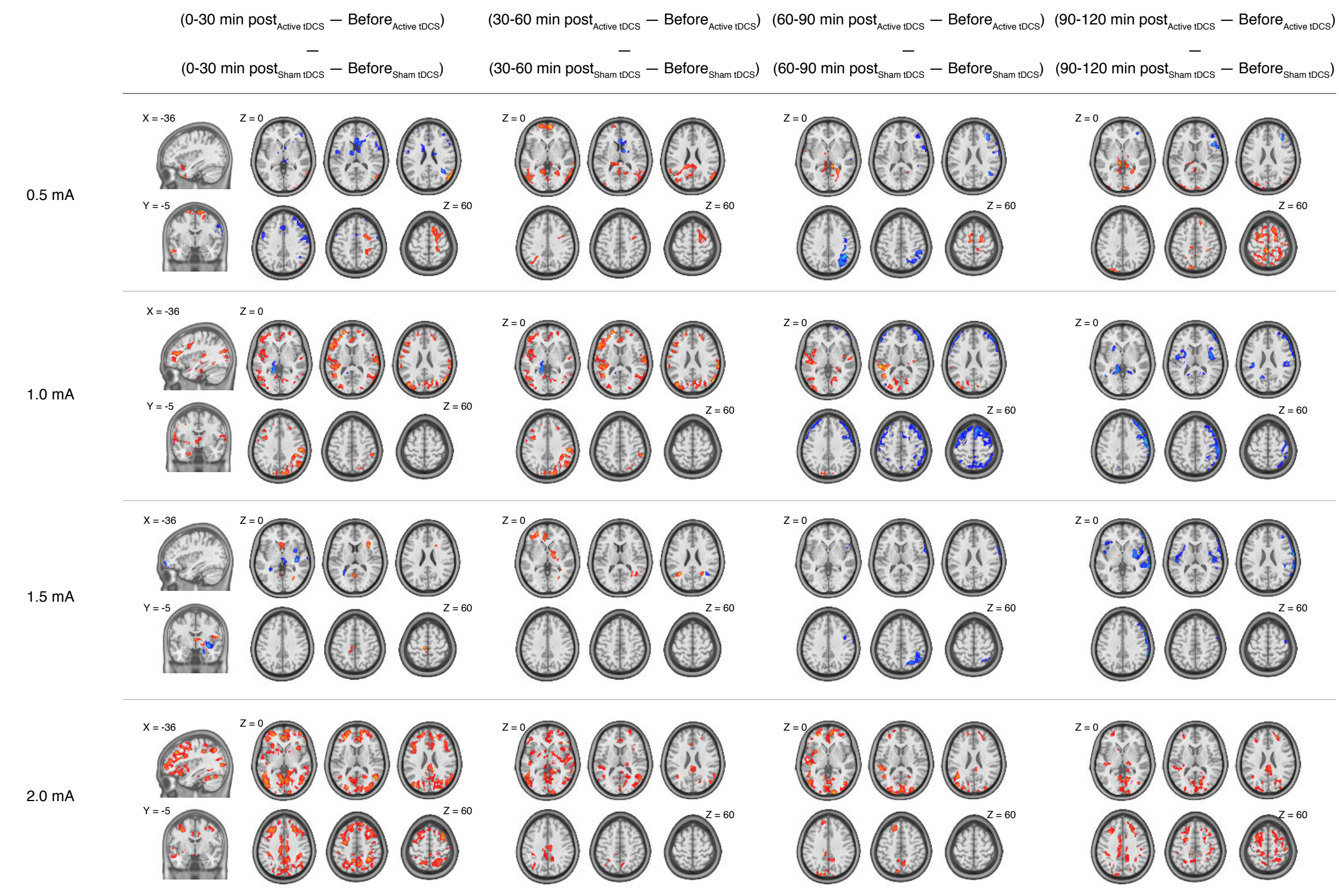


Figure 6. Cathodal tDCS-induced modulation of rCBF activity. For contrasts of main effects, red color indicates lower critical values and yellow indicates higher critical values. For paired contrasts, blue colors indicate a negative difference and red colors indicate a positive difference. A) A main effect of stimulation shows bilateral activation in the left superior temporal gyrus and the right supramarginal gyrus. B) Effect of time on $\mathrm{rCBF}$, collapsed over all stimulation intensities. Similar to findings with anodal tDCS (Figure 4), activations generally appeared widespread, and also overlapping with areas linked to the default mode network. C) A stimulation intensity $\mathrm{x}$ time interaction revealed a cluster of activation in the right frontal pole. D) Paired contrasts between active and sham tDCS intensities, collapsed over time, accompanied with corresponding effect sizes. Interestingly, $1.5 \mathrm{~mA}$ and $2.0 \mathrm{~mA}$ resulted in increased $\mathrm{CBF}$ in the frontal areas directly under or adjacent to the reference electrode.

A

Main Effect of Stimulation Intensity

Right Supramarginal Gyrus \& Left Superior Temporal Gyrus

$\mathrm{x}=64$
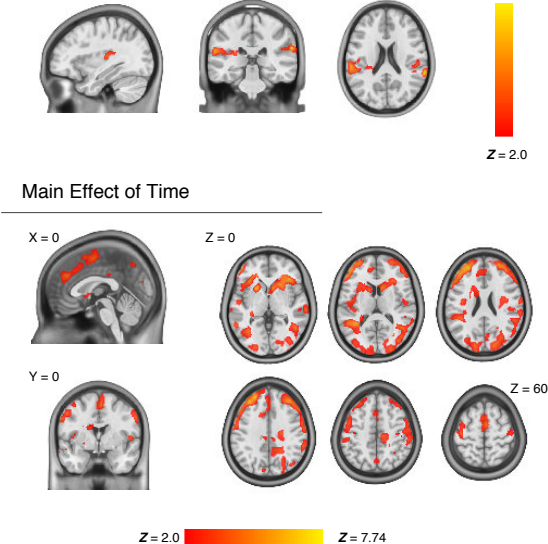

$z=2.0 \quad z=7.74$

C

Stimulation Intensity X Time Interaction

Right Frontal Pole

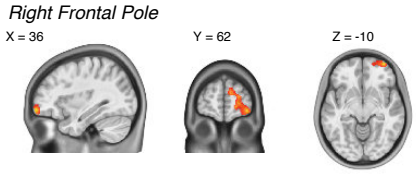

$z=2.0 \quad z=5.05$
D Main effect ${ }_{\text {Active tocs }}-$ Main effect $t_{\text {Sham tocs }}$
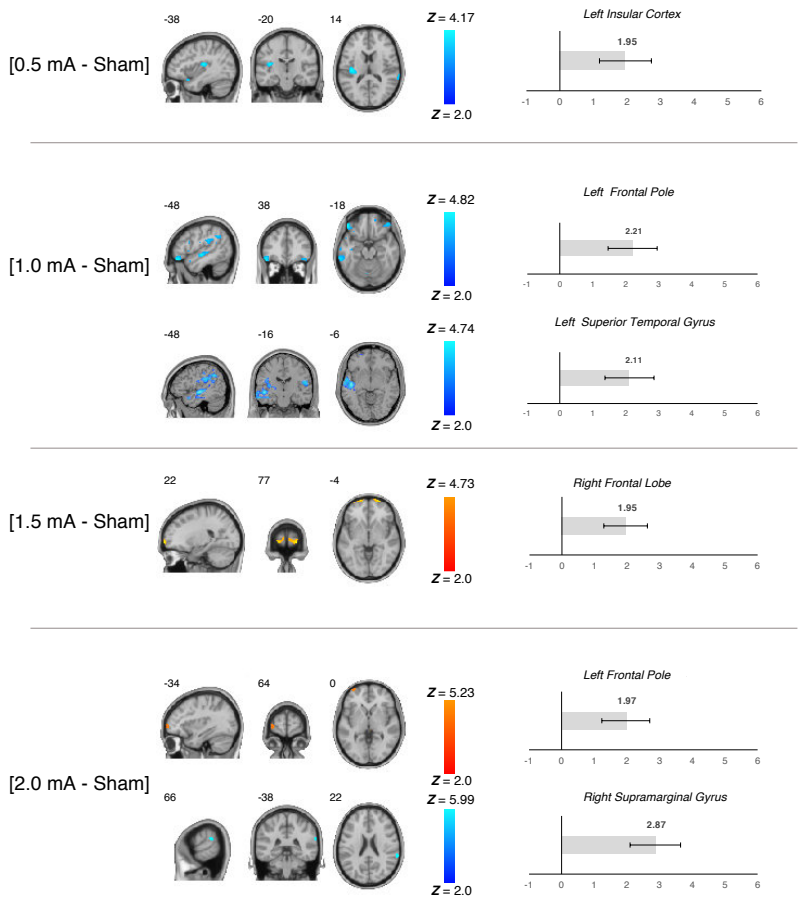
Figure 7. Exploration of time- and intensity-dependent alterations in rCBF following cathodal tDCS. Perfusion maps were binned into $30 \mathrm{~min}$ intervals, then normalized to baseline. Paired contrasts were conducted against sham tDCS. Red colors indicate a positive difference while blue colors indicate a negative difference. Maximum effects were contained in the the time range 30-90 min over all intensities. $1.0 \mathrm{~mA}$ and $2.0 \mathrm{~mA}$ induced notable decreases in rCBF over large basal areas in the left hemisphere. $0.5 \mathrm{~mA}$ and $1.5 \mathrm{~mA}$ resulted in relatively weaker decreased activation. 


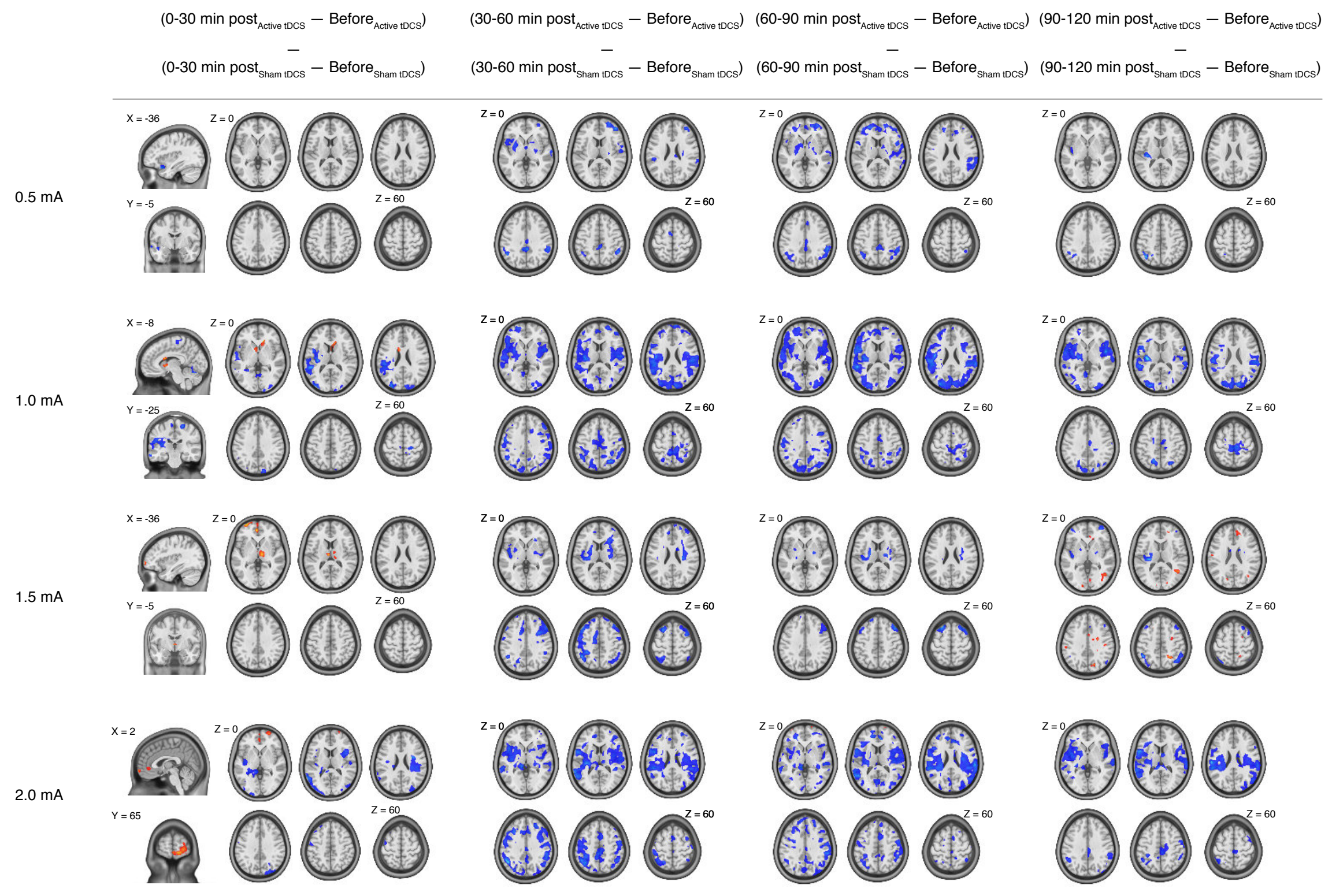


Figure 8. Seed based functional connectivity analysis of left M1 - Anodal tDCS. Functional connectivity by means of a seed based correlation analysis was conducted between the left precentral gyrus (M1) and the remaining cortical and subcortical areas which were parcellated by the AAL atlas. Red lines indicate significantly increased functional connectivity ( $p<0.05$, corrected) and blue lines represent decreased connectivity. A) A paired t-test contrast between active tDCS intensities to sham, collapsed over all timepoints, revealed mostly increased interhemispheric connectivity to motor, and sensorimotor-related areas. $0.5 \mathrm{~mA}$ also induced decreased connectivity to contralateral frontal areas. B) Paired contrasts between active and sham tDCS was further explored in the time dimension, grouped into $30 \mathrm{~min}$ intervals. Interestingly, inter-hemispheric connectivity between M1 to contralateral frontal and sensorimotor areas tended to be greatest during the early time periods with the lower intensities of 0.5 and $1.0 \mathrm{~mA}$, and more delayed with higher intensities of 1.5 and $2.0 \mathrm{~mA}$. 
B [During Stim. - BL]

[0 - $30 \mathrm{~min}-\mathrm{BL}]$

[30 - 60 min - BL]

[60 - 90 min - BL]

[90 - $120 \mathrm{~min}-\mathrm{BL}]$

[0.5 mA - Sham]
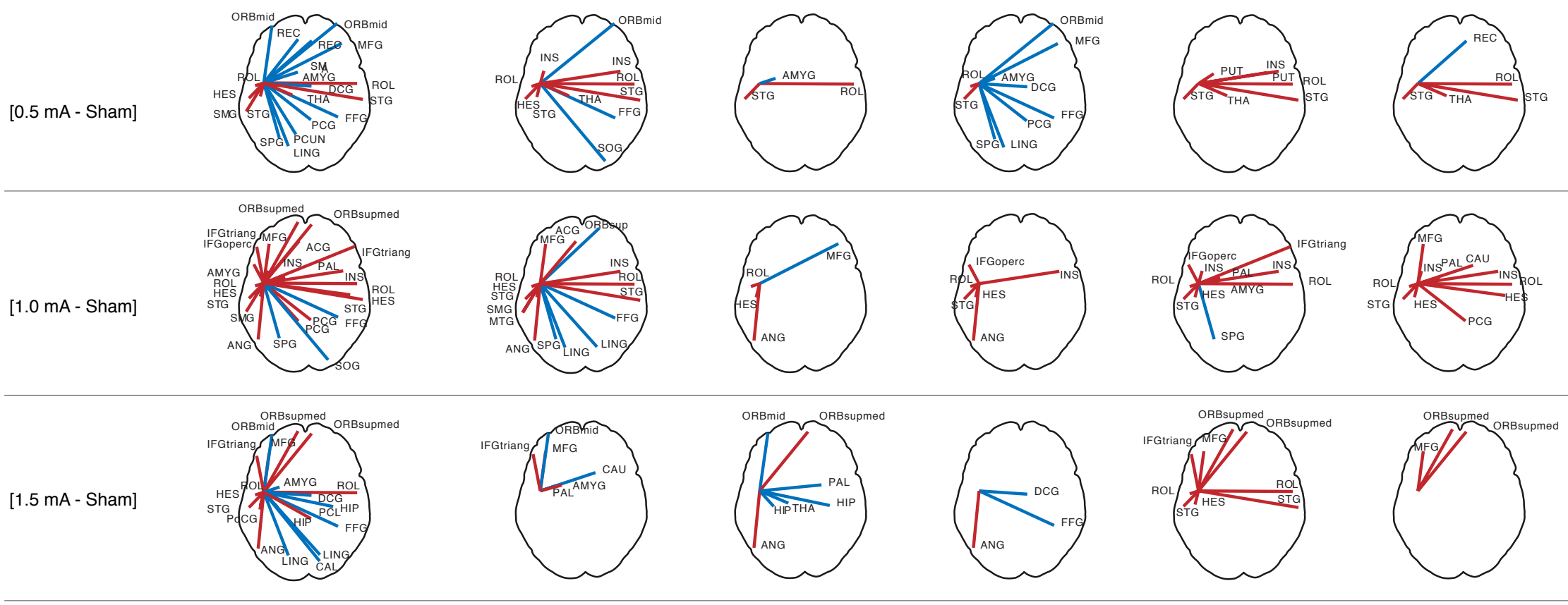

[2.0 mA - Sham]
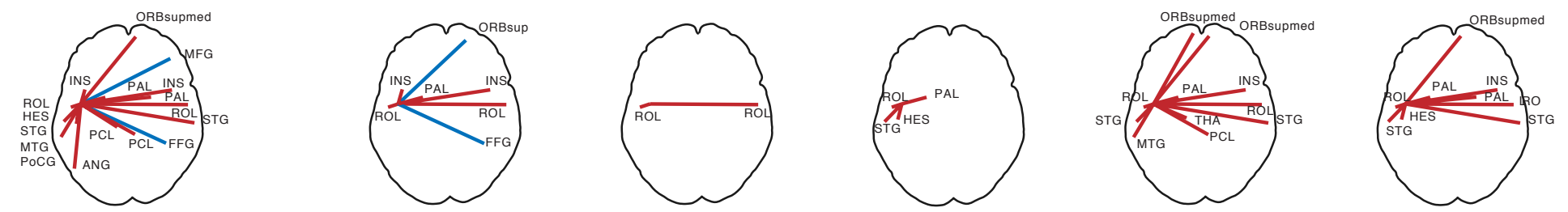
Figure 9 - Seed based functional connectivity analysis of left M1 - Cathodal tDCS. Functional connectivity by means of a seed based correlation analysis was conducted between the left precentral gyrus (M1) and the remaining cortical and subcortical areas which were parcellated by the AAL atlas. Red lines indicate significantly increased functional connectivity ( $\mathrm{p}<0.05$, corrected) and blue lines represent decreased connectivity. A) Paired t-test contrasts between active tDCS intensities to sham, collapsed over all time-points, revealed that lower intensities of 0.5 and $1.0 \mathrm{~mA}$ mainly decreased connectivity to contralateral motor, and sensorimotor-related areas whereas higher intensities of 1.5 and $2.0 \mathrm{~mA}$ resulted in increased connectivity to contralateral frontal regions. B) Paired contrasts between active and sham tDCS was further explored in the time dimension, grouped into $30 \mathrm{~min}$ intervals. Notably, $1.0 \mathrm{~mA}$ induced large-scale decreases in connectivity during the stimulation period, which attenuated over the remaining time course. 
[0 - 30 min - BL]

[30 - 60 min - BL]

[60 - 90 min - BL] [90 - $120 \mathrm{~min}-\mathrm{BL}]$

[0.5 mA - Sham]
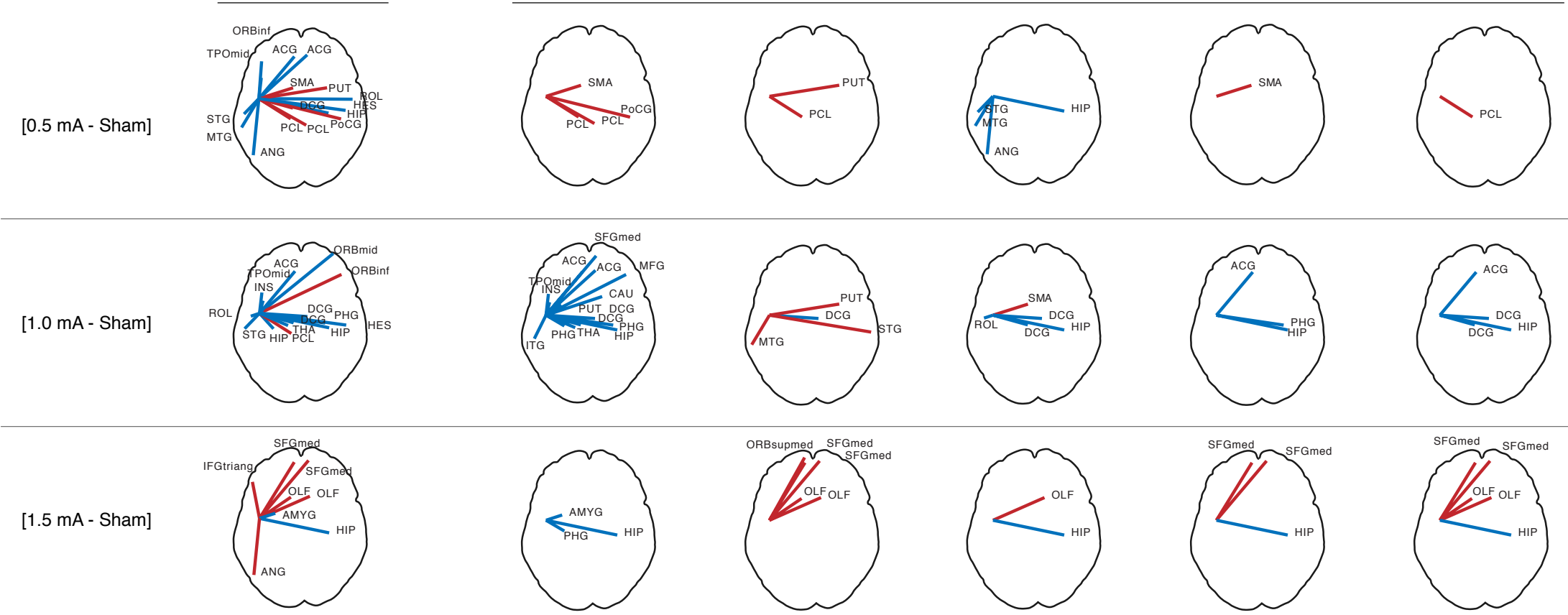

[2.0 mA - Sham]
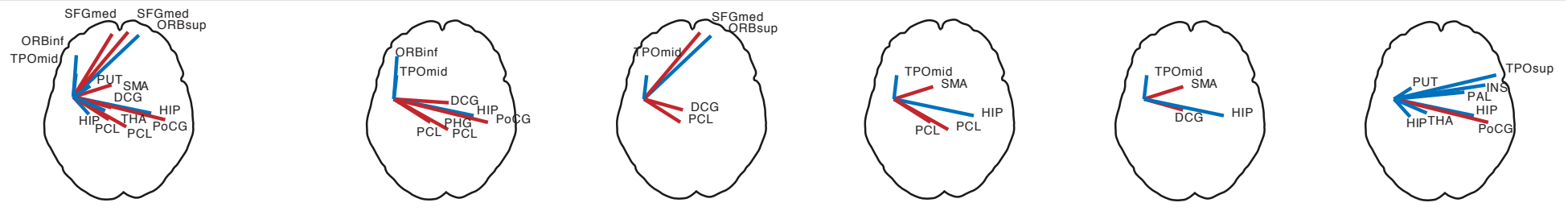
Figure 10 - Effect sizes of left M1 connectivity alterations between active and sham-tDCS. For each significant region implicated from an overall ANOVA in connectivity alterations (see Figures 8 and 9), an effect size was calculated. Regions were separated by hemispheres in order to qualitatively assess the extent of the effects. Mean effects sizes for each intensity were also calculated over all regions. A) For anodal tDCS, all intensities induced generally increases in connectivity, which were strongest in their effect with 1.0 and $2.0 \mathrm{~mA}$. B) For cathodal tDCS, $1.0 \mathrm{~mA}$ induced generally the strongest decrease in connectivity, with no difference between the left and right hemispheres.

\section{A Anodal tDCS}

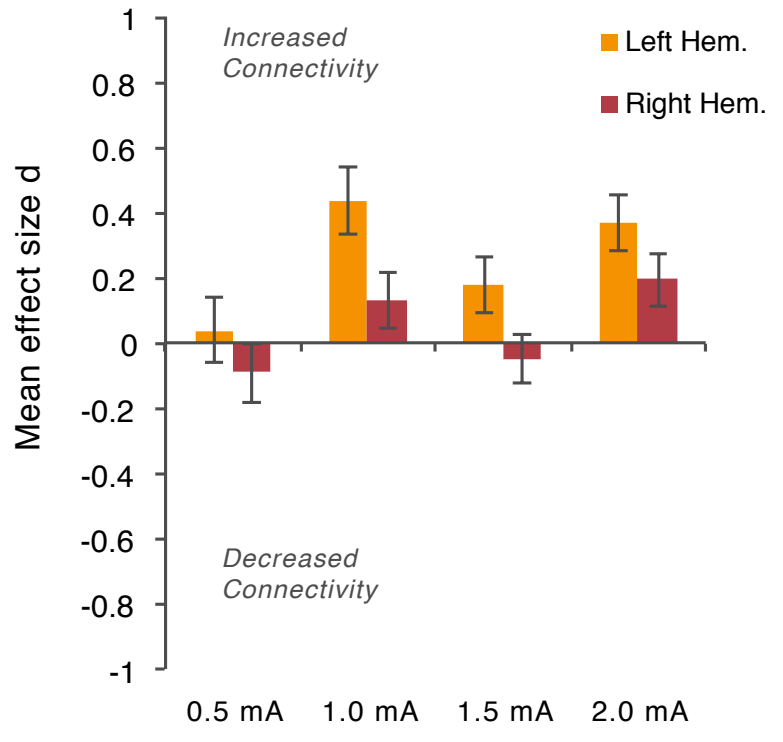

B Cathodal tDCS

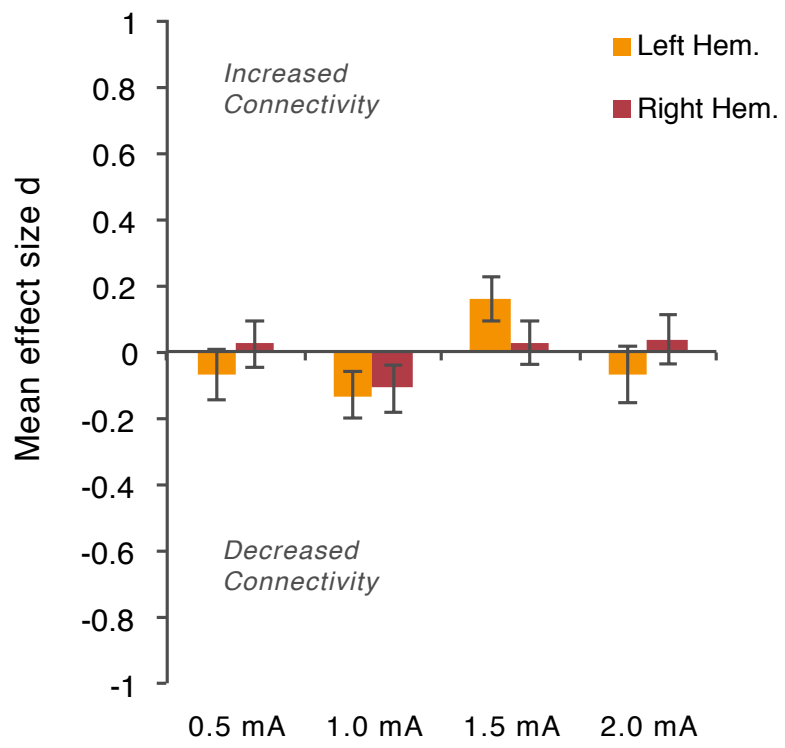


Figure 11 - Exploratory seed connectivity analyses of right frontal orbit. To follow up the findings of increased rCBF following cathodal tDCS in the contralateral frontal regions (see Figures 3 and 6), an exploratory connectivity analysis was conducted, setting the right frontal orbit as the seed. Connectivity differences after vs before tDCS show the influence of higher cathodal tDCS intensities of 1.5 and $2.0 \mathrm{~mA}$ in increasing the connectivity between the frontal orbital region with motor and visual cortical regions.

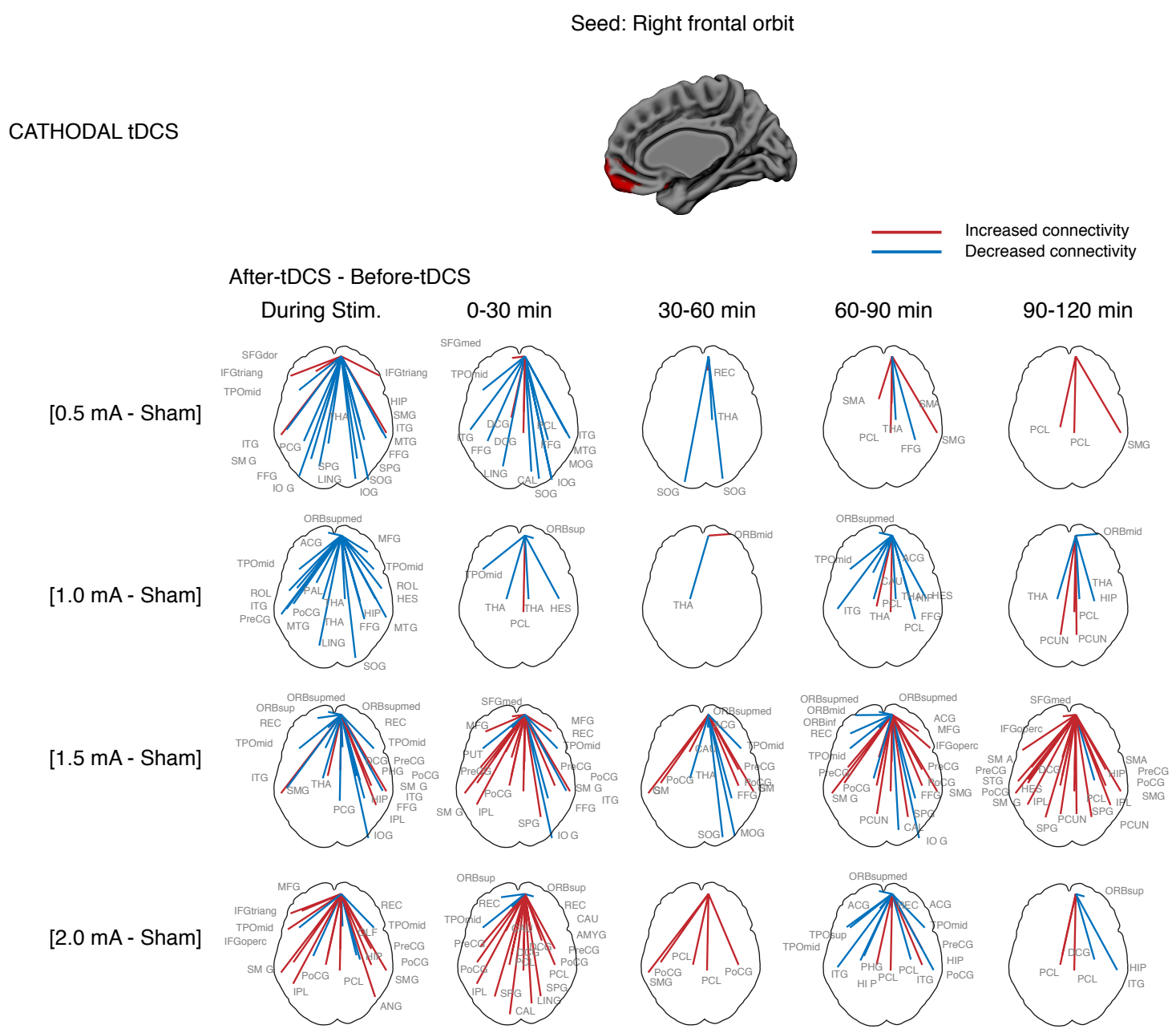


Figure 12: Influence of individual covariates on tDCS after-effects. In a series of regression analyses, after-effects of tDCS were compared to individual factors of age, baseline rCBF, gray matter M1 volume (GMB), electrode-tocortex distance (ECD) and TMS sensitivity, measured as the stimulus intensity needed to elicit a $1 \mathrm{mV}$ motor evoked potential of the right abductor digiti minimi muscle $(\mathrm{SI} 1 \mathrm{mV})$. The extent of the correlations is displayed as Manhattan plots and effect sizes. A) Anodal tDCS: gray matter volume was found to correlate with efficacy of $2.0 \mathrm{~mA}$ anodal $\mathrm{tDCS}$ ( $\mathrm{p}<0.05$, uncorrected). Moreover, although not significant, there was a tendency of this factor to linearly depend on the intensity of tDCS (lines are marked in red). Other factors had no significant relationship. B) Cathodal tDCS: There was no clear relationship between active tDCS intensities and individual covariates with after-effects in rCBF. In an overall analysis, gray matter volume and electrodecortex distance was found to be closely correlated with SI1mV (C,D), while there was no relationship between electrode-cortex distance and gray matter volume (E).
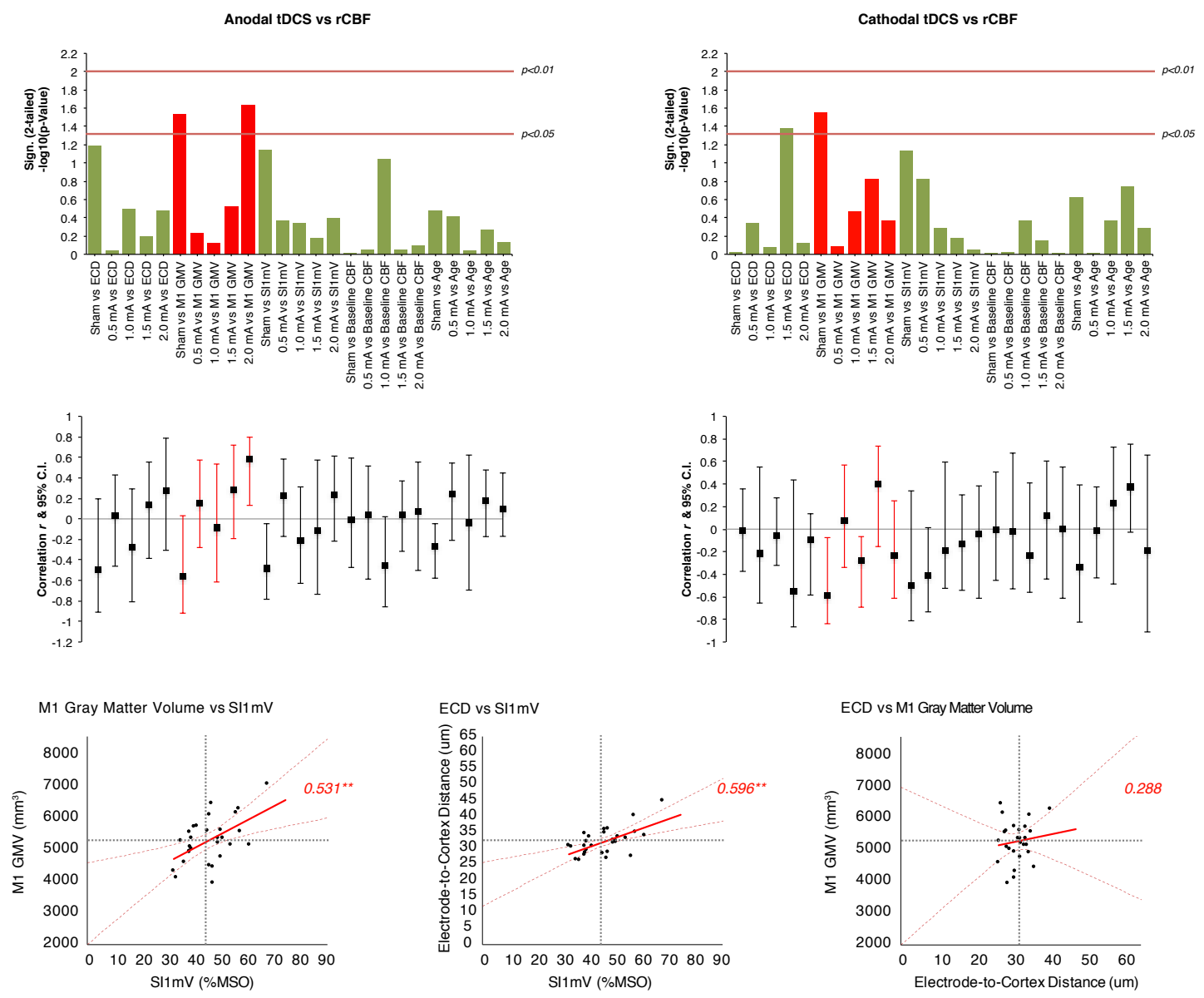
Exploring functional effects of tDCS-induced neuroplasticity in bimanual motor learning

Title: Investigating bimanual motor coordination in healthy young and older adults using EEG and transcranial direct current stimulation (tDCS)

Asif Jamil ${ }^{1,2,4}$, Koen Cuypers ${ }^{2,3}$, Michael A. Nitsche ${ }^{1,4,5}$, and Raf Meesen ${ }^{2,3}$

${ }^{1}$ University of Göttingen, Germany

${ }^{2}$ University of Hasselt, Belgium

${ }^{3}$ Katholieke University of Leuven, Belgium

${ }^{4}$ Leibniz Research Centre for Working Environment and Human Factors, Dortmund Germany

${ }^{5}$ University Medical Hospital Bergmannsheil, Bochum Germany

Corresponding author:

Asif Jamil

Dept. Psychology and Neurosciences

Leibniz Research Centre for Working Environment and Human Factors, Dortmund Germany

Ardeystr. 67

44139 Dortmund

jamil@ifado.de 


\begin{abstract}
Introduction: Accompanying the natural advancing of age is a decline in cognitive and motor functions, which can significantly impact the daily life activities in the elder demographic ( $>65 \mathrm{y})$. Such declines may involve altered neuroplasticity, due to changes in synaptic function and neurotransmission. Successful performance of complex motor tasks may also entail distinct patterns in motor cortical functional connectivity, which may also be subject to age related changes. On the other hand, recent work has shown that transcranial direct current stimulation (tDCS) may be a useful tool to restitute these altered mechanisms, and improve performance of motor skills.
\end{abstract}

Objectives: The present study first addresses the question of identifying physiological markers of age-related differences during acquisition of new bimanual motor control tasks, based on motor cortical functional connectivity using EEG. Second, the study assesses whether performance of complex bimanual motor skills can be improved in the elderly using tDCS.

Methods: Experiment 1: 43 healthy subjects (22 young/21 elderly) were recruited. Subjects performed the bimanual tracking task (BTT), which is a complex task requiring multiple cognitive domains, as well as the skilled use of in-phase and anti-phase movements, at various frequencies. Three blocks of the task were performed (180 total trials) while 32-channel EEG was recorded. Experiment 2: An additional 40 subjects (20 young/20 elderly) were recruited for evaluating whether right M1 anodal tDCS (1.0 mA, $20 \mathrm{~min}$ ) may improve performance in the task, particularly in the non-dominant left hand. The study was double-blinded, sham-controlled, and employed a randomized crossover design in order to assess tDCS-induced performance and functional connectivity differences between young and elderly groups.

Results: Experiment 1: Task performance in younger subjects was more accurate than in elderly. Younger subjects exhibited significantly stronger functional connectivity in the theta power band, which was also a reliable predictor for accurate performance of the task.

Experiment 2: ANOVA revealed a main effect of stimulation, which was significant between sessions in the elderly but not in young. Further exploratory analyses revealed significant improvements in both left and right hand coordination in real stimulation conditions for both groups of subjects.

Conclusion: We show that both functional connectivity and inter-limb kinematics underlying bimanual motor coordination are different between the young and elderly. We further show that a single session of tDCS applied to the motor cortex was able to significantly improve bimanual performance in both young and elderly. Although further studies are needed to optimize tDCS parameters for enhanced and prolonged effects, this non-invasive stimulation technique may be a viable tool in restituting and even further optimizing the learning of complex motor functions in the aging population. 


\section{Introduction}

The World Health Organization estimates that between 2010 and 2050, the number of people aged 65 and older is projected to increase worldwide by 188 percent. The average life expectancy is also expected to grow, having increased by 3 years since the last five years (ONU, 2015). Remarkable advancements in the daily lifestyle as well as in the quality of modern medicine have undoubtedly contributed a pivotal role in prolonging the average lifespan; however, these increasing trends also continue to underscore the need to develop more focused strategies in addressing age-related declines, particularly as they pertain to key cognitive and motor functions. Reports of age-related cognitive changes impacting the daily life activities have been documented across many domains, such as in attention, memory, planning, and other executive functions (Celsis, 2000; Zimerman \& Hummel, 2010). These alterations may be linked to complex physiological processes in the brain, such as decreases in white and gray matter integrity (Resnick et al., 2003; Salat et al., 2004), deteriorations in synaptic connections (Burke \& Barnes, 2006), and/or changes in the brain vasculature (Kannurpatti et al., 2010). Age-related decline in functions which involve complex interhemispheric interactions, such as bimanual motor coordination, have also been reported (Spirduso et al., 2005; Fujiyama et al., 2014), and suggest that alterations in interhemispheric connectivity may have an impact on the successful learning and performance of new bimanual motor skills, such as learning to type on a keyboard or to play the piano. This is of special importance, given that the quality of life in the elderly depend on the healthy functioning of the hand (Scherder et al., 2008; Zimerman \& Hummel, 2010).

On the other hand, recent developments in non-invasive brain stimulation have shown promise in externally inducing neural plasticity in the brain in a controlled manner (Pascual-Leone et al., 1994; Stefan et al., 2000; Nitsche \& Paulus, 2000). tDCS employs a weak electric current (1-2 mA) to induce a polarity-dependent shift in cortical excitability. Anodal tDCS delivered to the primary motor cortex has been shown to increase motor cortical excitability in humans, whereas cathodal tDCS decreases excitability (Nitsche \& Paulus, $2000,2001)$. A single application of anodal tDCS over M1 has been shown to induce transient performance improvements in various simple motor tasks (Galea and Celnik, 2009; Hunter et al., 2009; Reis et al., 2009; Stagg et al., 2011a,c), as well in visuo-motor learning A, implicit motor learning (Nitsche et al. 2003), enhancement of the non-dominant hand function (Boggio et al. 2006), and enhancement of the contralateral hand function (Vines et al. 2006). Thus, the technique offers the potential to restitute neural plasticity which may counter-act the decline in the motor functions in the elderly.

A recently developed motor learning paradigm to study properties of complex brain organization and interregional interaction is the bimanual tracking task (BTT), which requires both skilled motor performance, as well as cognitive control over both hands (Sisti et al., 2011; Beets et al., 2015). Participants are 
instructed to use both hands to rotate two dials at different cyclic frequencies or direction in order to follow a moving target on the screen. The task can be systematically programmed to be easier or harder depending on the relative frequency ratios of both dials, the in-phase or anti-phase relationship between the dials, and the selective sensitivity of one dial to move at a different frequency than the other, which could be used in order to evaluate hemisphere-specific control (Sisti et al., 2011).

In the present study, we were interested to explore the age-dependent differences in bimanual motor control, and also whether a single application of tDCS in elderly participants may influence the learning acquisition of the bimanual tracking task. To address these questions, we first conducted a pilot study composed of both young and elder healthy participants where task performance was combined with simultaneous EEG, in order to evaluate the behavioral and physiological extent of age-related differences. In the next experiment, using a separate cohort of young and older participants, we applied anodal or sham tDCS to the right M1 during the task performance, which has been shown previously to more prominently alter functional connectivity, and enhance motor function (Reis et al., 2009; Polanía et al., 2011). We hypothesized that the neuromodulatory intervention of tDCS would enhance the learning of the task, and that the elder may benefit from tDCS more than younger (Furuya et al., 2013).

\section{Materials and Methods \\ Subjects}

A total of 83 participants were collected over the span of two experiments. In the first pilot experiment, two groups of subjects were acquired: 21 older participants ( 9 females, mean age 70.54, SD 3.93 y) as well as 22 younger participants (12 females, mean age 26.45 , SD 3.32 y). For the second, main experiment, an additional two groups of subjects were acquired: 20 older participants ( 7 females, mean age 71.67, SD 3.58 y) and 20 younger participants (10 females, mean age 24.32, SD $2.55 \mathrm{y}$ ). Criteria for inclusion consisted of age requirement of 18-30 y or 65-77 y, right handedness (assessed by the Edinburgh Handedness Inventory - Oldfield, 1971), non-smokers, nonpregnant, no history of neurological or psychiatric disorders, no physical impairments affecting the usage of both hands, and no metal implants. For both experiments, all subjects were naïve to the motor task, and for the second experiment, all subjects were naïve to tDCS. All subjects provided their written informed consent and were compensated for participation. The study was approved by the institutional ethic committees of the University of Hasselt and KU Leuven, Belgium, and conformed to the guidelines established in the Declaration of Helsinki.

\section{Bimanual tracking task}

The objective of the BTT is to accurately track a moving target presented on a screen with a cursor by rotating two dials controlled by each hand. Participants were comfortably seated on a chair with both arms placed on a 
table under a small blinding hood, so that the movement of the hands are not visible while the subject is engaged with the task on a monitor.

In this task, two dials are used to perform the required bimanual movement that is displayed as a white dot moving along a line on the monitor. The left and right dials control the vertical and horizontal axes, respectively. Participants are instructed to trace the moving white dot along the line by simultaneous cyclical rotation of the dials. Real time visual feedback is provided by means of a red cursor displaying the actual tracking trajectory based on the coordination between the two hands. Unknown to the participant, the difficulty of the task is randomly varied between trials by changing the relative frequency ratio of the hand movements, which correspond to different slopes of the target trajectory (see Figure 1 for an overview). Trials commenced with a 2 -sec planning phase, during which subjects could see the required path they had to perform and then a 6-sec tracking phase, during which period the participant had to follow the moving target on the path. At the end of each trial, participants were guided by visual feedback to return their hand to a neutral position, in preparation for the next trial. The following frequency ratios were tested in the present study; 3:1, 2:1, $1: 1,1: 2$, and 1:3, where the first digit represents the ratio of the required speed of the left hand in relation to the right hand, the second digit.

\section{Electroencephalography}

With the exception of the first block in experiment 2 (the tDCS block), EEG was continuously recorded over the entire course of the task at the following 32 positions of the international 10-20 EEG system: Fp1, AF3, F7, F3, FC1, FC5, T7, C3, CP1, CP5, P7, P3, Pz, PO3, O1, Oz, O2, PO4, P4, P8, CP6, CP2, C4, T8, FC6, FC2, F4, F8, AF4, Fp2, Fz, and Cz. The sampling frequency was $500 \mathrm{~Hz}$ and the electrode impedance was monitored throughout the experiment to be less than $5 \mathrm{kOhms}$.

\section{Transcranial Direct Current Stimulation}

tDCS was delivered by a battery driven constant current stimulation (neuroCare $\mathrm{GmbH}$, Ilmenau, Germany) through a pair of square rubber electrodes $(35 \mathrm{~cm} 2$ target electrode and $100 \mathrm{~cm} 2$ reference electrode). The target electrode was placed over the $\mathrm{C} 4$ position, which was located and marked on the scalp using the 10-20 EEG cap. The reference electrode was placed over the contralateral left frontal orbit. Our rationale for targeting the non-dominant hemisphere was based on the results of our pilot experiment, where we observed that left hand performance was much worse than right hand performance across all young and older subjects (all of whom were righthanded). Moreover, decreased functional connectivity of the non-dominant hemisphere-in terms of weaker synchronization-might be expected (Amunts et al., 2000), and applying anodal tDCS may increase this connectivity (Polania et al. 2011). Duration of the stimulation coincided with the duration of the first BTT block, which lasted 20 minutes. An anodal tDCS intensity of $1.0 \mathrm{~mA}$ was chosen, based on the premise that this intensity induces modifications in cortical excitability lasting up to $60 \mathrm{~min}$ (Nitsche et al., 2008). Furthermore, it is not clear whether intensities higher than $1.0 \mathrm{~mA}$ 
may be functionally efficient in targeting only the right motor cortex, as higher intensities of 1.5 and $2.0 \mathrm{~mA}$ have been shown to induce alterations under the reference electrode and also in contralateral motor areas (Jamil et al. - in submission). Anodal or sham tDCS was delivered using a 40-sec ramp at the beginning and end of the stimulation period. Both the participant and the researcher were blind to the type of tDCS. Following the end of each session, participants were asked to document on a questionnaire whether they believed they received real tDCS ("yes" or "no").

\section{Experimental design}

In the first experiment, 22 young and 21 elder participants took part in a single session of EEG combined with the BTT paradigm. Participants were provided with a minimal verbal description of the task without any disclosure of the difficulty constraints that could be expected, and each participant was able to practice and familiarize with the concept of the task over 10 randomized trials before the first experimental block. Three blocks were designed, where each block contained 60 trials in a randomized sequence of varying difficulties of the task. In order to minimize artefactual EMG in the EEG, participants were asked to maintain a relaxed posture in the reclined chair and keep their head stationary on a head rest throughout the task. Following the end of each block, which lasted approximately $15 \mathrm{~min}$, a $5 \mathrm{~min}$ rest was provisioned before the next block began.

In the second experiment, a new cohort of 20 young and 20 elder participants were recruited to take part in two sessions of the task. A counter balanced and randomized cross-over design was implemented such that each participant attended a session of real tDCS and sham tDCS in a balanced ordering over the group, with at least a 10-day gap between the two sessions as a wash out for task consolidation. Similar to the first experiment, participants were verbally instructed and allowed to familiarize with the concept of the task for 10 trials before beginning. Three standard experimental blocks were designed as previous, however with each block containing 40 trials instead of 60 , in order to reduce the total time of each task block to 10 min and also minimize an over-learning of the task, which could interfere with the second session (see experiment 1 results). To assess the extent of consolidation of the task, we included an additional final fourth block in this experiment, during which participants were asked to complete 14 trials without receiving visual feedback. In other words, participants were able to see only the target moving on the screen, and thus had to rely on an internal reference for correctness (possibly a somatosensory based model) to accomplish the task (Beets et al., 2015). Real or sham tDCS was delivered during the respective session during only the first and second block (20 min), and EEG was recorded during the third (10 min) and fourth (2 min) block.

\section{Statistical processing}

Kinematic analysis

BTT performance data were recorded with Labview (8.5) software (National Instruments, Austin, TX, USA). The $\mathrm{x}$ and y positions of the moving target 
and the subjects' cursor were sampled at $100 \mathrm{~Hz}$. Offline analysis was carried out using Matlab R2015b and statistical analysis was conducted in SPSS version 22. Accuracy was measured by calculating the sum of the average subject-to-target error and the average subject-to-track error. In other words, for each trial, the target and track error was measured as the Euclidian distance between the target or track and the cursor position at each point in time and then averaged. Outlier trials $(z>3)$ were discarded from the analysis. For each subject, and for each block of the task, trials were grouped by the task condition and the performance was averaged together. At the group level, the mean values were entered into a 3 (blocks) x 5 (task conditions) repeated measures ANOVA with age group as a between subject factor. Mauchly's Test was used to check for non-sphericity, and Greenhouse-Geisser correction was applied if necessary. Significant effects within the groups were evaluated posthoc using two-tailed paired t-tests. Age-group comparisons were conducted using unpaired t-tests (two-tailed, $\mathrm{p}<0.05$, uncorrected).

\section{EEG Analysis}

Preprocessing

Analysis of the EEG data was performed using a script based on the Fieldtrip library in MATLAB. The BTT encoded virtual triggers to the EEG recording, which allowed the data to be split into respective task trials. Offline preprocessing of the EEG was performed in MATLAB, which included a band pass filtering between $0.1-125 \mathrm{~Hz}, 50$ and $100 \mathrm{~Hz}$ notch filtering, linear detrending, down-sampling to $250 \mathrm{~Hz}$, and then removal of EOG and EMG artefacts based on a visual inspection of artifacts extracted from an independent component analysis (method 'runica', implemented in Fieldtrip). Furthermore, individual trials were visually inspected and artefactual trials containing high variance $(\mathrm{z}>3$ ) were discarded (which ranged $\sim 8 \%$ of the data per block).

\section{Functional connectivity analysis}

Channel level connectivity between the 32 EEG electrodes was assessed by means of the weighted phase lag index (WPLI), a measure which represents the absolute value of the imaginary component of the cross-spectrum, is debiased to sample size, and is less sensitive to volume conduction noise (Vinck et al., 2011). WPLI between two channels is calculated as follows:

$$
W P L I=\frac{\sum_{j=1}^{N} \sum_{k \neq j} \mathfrak{I}\left\{X_{j}\right\} \mathfrak{I}\left\{X_{k}\right\}}{\sum_{j=1}^{N} \sum_{k \neq j}\left|\mathfrak{I}\left\{X_{j}\right\} \mathfrak{I}\left\{X_{k}\right\}\right|^{\prime}}
$$

where $\mathrm{N}$ is the number of trials and $\mathfrak{I}\{$.$\} represents the imaginary part of the$ cross-spectrum (Vinck et al., 2011). The WPLI was calculated across six frequency bands of interest, Delta $(1-4 \mathrm{~Hz})$, Theta $(4-8 \mathrm{~Hz})$, Alpha $(8-13 \mathrm{~Hz})$, Beta $(13-30 \mathrm{~Hz})$, Low Gamma $(30-45 \mathrm{~Hz})$ and High Gamma $(55-90 \mathrm{~Hz})$, and separately across trials for each difficulty level of the task. We first analyzed the mean connectivity strength (i.e., the average WPLI over all $32 \times 32$ channel combinations) to discriminate any differences between the task conditions and frequency bands. Repeated measure ANOVAs were conducted to discern the main effects of age and task-specific factors in experiment 1, and for the 
additional factor of stimulation in experiment 2. To investigate topological differences, network connectivity analysis was conducted within the framework of statistical parametric network (SPN) analyses as described by (Ginestet \& Simmons, 2011). Briefly, at the group level, the mean 32x32 WPLI matrix for each condition was aggregated over the subjects into a $3 \mathrm{D}$ matrix $(32 \times 32 \times n)$ and a one sample t-test was performed along the third dimension at each element in order to reveal a group mean connectivity graph. Pair-wise connections in this graph ("edges") were thresholded based on inference testing of the unpaired or paired $t$-test statistic $(\mathrm{p}<0.05)$. The alpha probability for Type II error was corrected for multiple comparisons using the false discovery rate, which provides a reasonable balance between control of type II error and sensitivity of detecting robust effects (Benjamini \& Hochberg, 1995; Ginestet et al., 2011). For experiment 2, we followed the same procedure, this time calculating differential SPN graphs between real and active tDCS sessions for each task condition by means of paired t-tests. The key advantage of using this method is that the quasilinearity of thresholding connectivity matrices for significant connections is addressed because inference is drawn from the connectivity coefficients themselves, and an arbitrary threshold is traded off for a specific significance level (Ginestet et al. 2011). This approach has been implemented in various studies using network analysis for the comparison between a group of subjects and a group of controls (Richiardi et al., 2011; Fornito et al., 2013).

\section{Results}

\section{Experiment 1}

The repeated measures ANOVA revealed a main effect of Age $(\mathrm{F}=17.005$, $\mathrm{df}=1, \mathrm{p}<0.001)$, Block $(\mathrm{F}=42.587, \mathrm{df}=2, \mathrm{p}<0.001)$, Task Condition $(\mathrm{F}=21.996$, $\mathrm{df}=4, \mathrm{p}<0.001)$, and Task Condition $\mathrm{x}$ Age $(\mathrm{F}=2.500, \mathrm{df}=4, \mathrm{p}=0.047)$ (Table 1). Post hoc unpaired sample t-tests between age groups revealed much poorer performance (higher error) in the elderly as compared to the young, present across all blocks and conditions (all values of $\mathrm{P}<0.05$ ) (Figure 2). Nevertheless, the tendency to improve over the course of the task is clearly apparent for both age groups. We also calculated the average learning rate (slope of the performance lines) and found that the elderly tended to improve performance in the task at a faster rate (average slope for elderly $=-6.4$, young $=-4.44$ ), however it is not clear whether ceiling effects may have prevented further improvement in the young.

Overall, connectivity in the low and high gamma range $(30-90 \mathrm{~Hz})$ during task performance was greater in younger participants as compared to elderly (Figure 3A). To investigate whether these age-dependent connectivity differences may have predicted more accurate performance in the task, we regressed subject-specific task performance against the respective connectivity matrix, controlling for the task condition, so that we could identify which pairs of EEG channels corresponded to more accurate performance in this frequency range. Figure $3 \mathrm{C}$ illustrates the findings, in which a relationship between the active hand task in the condition and increased connectivity in the associated (contralateral) hemisphere could be observed. In other words, 
conditions in which the right hand required greater activation (1:2 and 1:3) depended on the stronger connectivity between the left motor, frontal and adjacent channels. Similarly, right motor connectivity predicted better performance in conditions in which the left hand required greater involvement $(r=-0.23, p=0.01$; Figure $3 C-D)$.

\section{Experiment 2}

All subjects tolerated the stimulation conditions without any adverse effect. Overall, participant blinding was effective, given that only $56 \%$ of all responses were correct guesses, and thus not better than pure chance $\left(\chi^{2}=10.4, \mathrm{p}>0.05\right)$. An overall ANOVA revealed a main effect of stimulation $(\mathrm{F}=4.997, \mathrm{df}=1$, $\mathrm{p}=0.032)$, a main effect of age $(\mathrm{F}=29.261, \mathrm{df}=1, \mathrm{p}<0.001)$, a main effect of task condition $(\mathrm{F}=33.957, \mathrm{df}=6, \mathrm{p}<0.001)$, and a main effect of block $(\mathrm{F}=42.432$, $\mathrm{df}=2, \mathrm{p}<0.001)$. Post hoc analyses revealed a tendency for task improvements in both left and right hand coordination following all real tDCS conditions (Figure 4A). A significant between-condition difference was observed in the elderly group with a significantly improved performance in the left hand 2:1 condition $(\mathrm{p}=0.03)$. Younger subjects tended to improve performance as well, although ceilings effects may have been a constraint. To assess whether tDCS may have affected task consolidation (Beets et al., 2015), we asked participants to complete a final short block of trials in which visual feedback was withheld. A one-way ANOVA did not yield a main effect of stimulation $(\mathrm{F}=2.989, \mathrm{df}=1$, $\mathrm{p}=0.092)$, although we observed a main effect of task condition $(\mathrm{F}=25.220$, $\mathrm{df}=6, \mathrm{p}<0.001)$. As seen from Figure $4 \mathrm{~B}$, there was a small tendency of improved task performance during the no-feedback block over both age groups and all task conditions.

With regard to EEG alterations, our analysis revealed that the elderly had a tendency for an increase in functional connectivity after real tDCS, which was most pronounced in the gamma range (Figure 5). To assess the topology of the tDCS-induced differences, we contrasted for each group the real tDCS condition with the sham condition, plotting only those edges which survived an FDR adjusted multiple comparison correction (see methods). As shown in Figure 5, older participants showed a tDCS induced increase in right motor related areas within the high gamma frequency band for the left hand task conditions. In the right hand task conditions, bilateral frontal connectivity also increased for younger participants, whereas no clear changes were observed for older participants. Lower frequency connectivity in the theta and alpha range decreased after tDCS for both groups.

\section{Discussion}

Age-dependent differences during bimanual motor learning

The present study investigated age-related differences in the performance of a complex bimanual motor task. We observed clear differences in kinematics between the young and elderly as they performed the task. In line with our expectation of age-related decline in bimanual motor performance, the elderly performed less accurately in all task conditions, although they showed the 
ability to improve over the course of the session at a much faster rate as compared to the young. For both groups of participants, those task conditions which required higher involvement of the left hand (frequency ratios 3:1, 2:1) proved more difficult than those which required the right hand (ratios 1:2, 1:3), which is in general agreement with previous studies (Sisti et al., 2011; Beets et al., 2015; Fujiyama et al., 2016), and enforces the notion that hand dominance influences bimanual coordination (Murphy \& Peters, 1994; Amazeen et al., 1997; Franz et al., 2002).

EEG further revealed large-scale functional connectivity differences in the performance of the task. On the whole, elderly participants exhibited greater bilateral frontal connectivity compared to younger participants, in accordance with previous studies which have documented more lateralized connectivity in the young (Lustig et al., 2009; Seidler et al., 2010). It has been proposed that the additional bilateral activation is the result of the brain to "compensate" for the inefficient circuits which are normally required to complete the task (Cabeza et al., 2002). The role of altered functional circuits was recently shown in a study of the same task, where dual-site transcranial magnetic stimulation (TMS) was used to perturb interhemispheric interactions between the left dorsolateral prefrontal cortex (DLPFC) the left dorsal premotor cortex (PMd) and the right M1. Consistent with the aforementioned hypothesis, it was shown that these perturbations interacted with the accurate motor performance of elderly in the task (Fujiyama et al., 2016). Besides functional aspects, these differences may also be associated with impaired structural connectivity, such as decline in white matter integrity (Bennett \& Madden, 2014). Interestingly, it was recently demonstrated by a structural imaging study that bimanual motor performance was associated with changes in microstructural organization of the corpus callosum sub regions connecting homologous brain areas, include the two motor cortices (Serbruyns et al., 2013).

\section{Anodal tDCS over the right M1 enhances bimanual motor performance}

We found that applying $1.0 \mathrm{~mA}$ anodal tDCS to the right M1 in the elderly led to significant short-term improvements in bimanual motor performance, which was especially pronounced for conditions requiring the left-hand involvement. Besides a small tendency, the enhancement in performance observed in young participants was not significant.

The findings observed here are similar to previous studies reporting improvement in motor skill acquisition following tDCS (Nitsche et al., 2003; Boggio et al., 2006; Vines et al., 2006; Reis et al., 2009; Stagg et al., 2011). Plasticity induced during motor training is based on processes similar to long term potentiation (LTP), and also results in modification of cortical networks following stimulation. We observed that tDCS tended to enhance not only skill acquisition, but also skill consolidation, as indexed by the improved task performance in which no visual feedback was provided. Moreover, tDCS tended to increase functional connectivity to hemisphere-specific motor areas (Figure 5). These findings are likely based on the proposed mechanism in which anodal tDCS induces excitability alterations in the sensory-motor cortex (Nitsche \& Paulus, 2000, 2001), leading to after-effects which 
strengthen horizontal neuronal synaptic connections, necessary for motor memory formation (Harms et al., 2008; Xu et al., 2009). Although these changes appeared to coincide with modest alterations in functional connectivity, as observed with EEG following tDCS (Figure 5), the results do not necessarily imply that the primary motor cortex is the area in which bimanual motor coordination primarily takes place; a complex neural network also involving the supplementary motor area (SMA), the cingulate motor area, basal ganglia and cerebellum is likely involved (Swinnen \& Wenderoth, 2004). A key component in this complex motor task is the involvement of the DLPFC, which was shown in a recent fMRI study to decrease in activation after five days of training of a bimanual motor task (Beets et al., 2015).

The lack of enhanced skill acquisition in the younger group is also interesting, although not completely unexpected. One reason is that it cannot be completely ruled out that increases in performance were constrained by a ceiling effect, which may have prevented any room for further improvement. This is partly evident when observing the average block-to-block performance improvement trend, which was much higher for elderly participants than the young ( $6.40 \%$ vs $4.44 \%$, respectively), and which tended to stabilize between the second and third block across most of the task conditions. Our attempts to make the task more difficult by reducing the number of trials was therefore only effective in reducing overall performance, but not in altering change in performance over a time period. In this regard, further studies would be needed to obtain the optimal testing time which reliably indexes a subject's performance, as well as the change in performance over an adequate period. Interestingly, performance of younger participants in task conditions which required the right hand tended to improve more as compared to the conditions which required the left (Figure 4A). This tendency also coincided with greater increases in the functional connectivity between left M1 and contralateral frontal regions (Figures 5 and 6). One explanation could be that right $\mathrm{M} 1 \mathrm{tDCS}$ enhanced the contralateral left M1 via homotopic mechanisms, resulting in increased excitability to this region (Pellicciari et al., 2013). However, this theory is speculative at best, and further research would be needed to ascertain if such a finding continues to hold over a longer period of task training.

A further question is whether age-dependent differences exist in the association between movement timing and performance accuracy, and whether tDCS may have altered the speed accuracy function within performers at different levels on the dichotomy (Reis et al. 2009). Recent studies investigating the neurophysiological basis for rhythmic timing control have linked the role of the cerebellum in maintaining temporal accuracy (Spencer \& Ivry, 2005) as well as interhemispheric interactions through the corpus callosum (Kennerley et al., 2002; Ridderikhoff, 2005), which may be relevantly affected by aging (Perceval et al., 2016). Newer metrics which make use of the speed accuracy relationship may be useful in order to investigate whether tDCS affects these processes (Reis et al., 2009).

Conclusion 
Age-related decline in cognitive and motor functions can critically affect daily life activities (Logsdon et al., 2002; Kannus et al., 2005). Here, our finding that a single session of tDCS was able to improve performance in a complex bimanual motor control task in elderly participants suggests that non-invasive brain stimulation may be a useful tool to boost functionally impaired motor learning processes. We further observed that associated enhancements appeared to be correlated with alterations in functional connectivity between the motor areas. Further research, perhaps using higher resolution EEG, or a combined TMS-EEG approach would be useful in understanding the precise mechanisms underlying performance gains. Moreover, this information will be indispensable for refining non invasive brain stimulation protocols for further application in both healthy and clinical populations. 


\section{Tables and Figures}

Table 1: Results of the repeated measures ANOVA for assessment of kinematics. An ANOVA was conducted to assess the main experimental factors of age group, task condition, and task block in the first experiment, and the additional factor stimulation in the second experiment. Age group was modeled as a between-subject factor. The dependent measure was the error in the subject's position relative to the track and the target (see methods). In experiment 1, age group was found to have a significant effect, which also interacted with the task condition. In experiment 2 , stimulation had a significant effect, which did not interact with age group.

\begin{tabular}{|c|c|c|c|c|}
\hline Experiment & Factor & $F$ & $D F$ & $\boldsymbol{P}$ \\
\hline \multirow[t]{6}{*}{1} & Block & 42.587 & 2 & $<0.001^{\star *}$ \\
\hline & Task Condition & 21.996 & 4 & $<0.001^{\star *}$ \\
\hline & Age Group & 17.005 & 1 & $<0.001^{\star *}$ \\
\hline & $\begin{array}{l}\text { Block }{ }^{*} \text { Age } \\
\text { Group }\end{array}$ & 2.533 & 2 & 0.088 \\
\hline & $\begin{array}{l}\text { Task condition * } \\
\text { Age group }\end{array}$ & 2.500 & 4 & $0.047^{\star}$ \\
\hline & $\begin{array}{l}\text { Block }{ }^{*} \text { Task } \\
\text { condition }{ }^{*} \text { Age } \\
\text { group }\end{array}$ & 0.487 & 8 & 0.865 \\
\hline \multirow[t]{12}{*}{2} & Stimulation & 4.997 & 1 & $0.032^{\star}$ \\
\hline & Task Condition & 33.957 & 6 & $<0.001^{\star *}$ \\
\hline & Age Group & 29.261 & 1 & $<0.001^{\star *}$ \\
\hline & Block & 42.432 & 2 & $<0.001^{\star *}$ \\
\hline & $\begin{array}{l}\text { Stimulation * Age } \\
\text { Group }\end{array}$ & 0.858 & 1 & 0.360 \\
\hline & $\begin{array}{l}\text { Stimulation * } \\
\text { Task Condition }\end{array}$ & 0.998 & 6 & 0.427 \\
\hline & $\begin{array}{l}\text { Stimulation * } \\
\text { Block }\end{array}$ & 0.486 & 2 & 0.617 \\
\hline & $\begin{array}{l}\text { Age Group }{ }^{*} \text { Task } \\
\text { Condition }\end{array}$ & 2.036 & 6 & 0.062 \\
\hline & $\begin{array}{l}\text { Age Group * } \\
\text { Block }\end{array}$ & 1.792 & 2 & 0.174 \\
\hline & $\begin{array}{l}\text { Stimulation }{ }^{*} \\
\text { Task Condition }{ }^{*} \\
\text { Age Group }\end{array}$ & 0.438 & 6 & 0.853 \\
\hline & $\begin{array}{l}\text { Stimulation }{ }^{*} \\
\text { Block }^{*} \text { Age } \\
\text { Group }\end{array}$ & 0.557 & 2 & 0.575 \\
\hline & $\begin{array}{l}\text { Task Condition * } \\
\text { Block }{ }^{\star} \text { Age } \\
\text { Group }\end{array}$ & 2.100 & 12 & $0.016^{*}$ \\
\hline
\end{tabular}


Stimulation *

0.663

Task Condition *

Block * Age

Group
12

0.788 
Table 2: Results of the repeated measures ANOVA in assessment of EEG connectivity. An ANOVA was conducted to assess the main factors of age group, task condition, and frequency band in the first experiment, and the additional factor stimulation in the second experiment. Age group was modeled as a between-subject factor. The dependent measure was the weighted phase lag index (WPLI) (see methods). In experiment 1, the main effect of age group was not significant. In experiment 2 , stimulation did not have a significant effect. Age group was a significant factor, which however did not interact with stimulation.

\begin{tabular}{|c|c|c|c|c|}
\hline Experiment & Factor & $F$ & $D F$ & $\boldsymbol{P}$ \\
\hline \multirow[t]{6}{*}{1} & Age & 0.896 & 1 & 0.353 \\
\hline & Task Condition & 0.213 & 4 & 0.649 \\
\hline & Frequency & 3.481 & 5 & $<0.001^{\star *}$ \\
\hline & $\begin{array}{l}\text { Age }{ }^{*} \text { Task } \\
\text { condition }\end{array}$ & 0.904 & 4 & 0.351 \\
\hline & Age $^{\star}$ Frequency & 0.301 & 5 & 0.876 \\
\hline & $\begin{array}{l}\text { Task * Frequency } \\
\star \text { Age }\end{array}$ & 0.474 & 20 & 0.755 \\
\hline \multirow[t]{13}{*}{2} & Stimulation & 0.0677 & 1 & 0.416 \\
\hline & Task Condition & 0.188 & 4 & 0.667 \\
\hline & Age Group & 7.486 & 1 & $0.009^{*}$ \\
\hline & Frequency & 3.481 & 5 & $0.005^{\star}$ \\
\hline & Frequency ${ }^{*}$ Age & 0.463 & 4 & 0.804 \\
\hline & Stimulation ${ }^{\star}$ Age & 0.287 & 1 & 0.595 \\
\hline & $\begin{array}{l}\text { Stimulation }{ }^{*} \\
\text { Task Condition }\end{array}$ & 0.225 & 4 & 0.638 \\
\hline & $\begin{array}{l}\text { Stimulation }{ }^{*} \\
\text { Frequency }\end{array}$ & 1.028 & 5 & 0.403 \\
\hline & $\begin{array}{l}\text { Age Group }{ }^{*} \text { Task } \\
\text { Condition }\end{array}$ & 0.058 & 4 & 0.811 \\
\hline & $\begin{array}{l}\text { Stimulation }{ }^{*} \\
\text { Frequency }{ }^{*} \text { Age }\end{array}$ & 1.960 & 5 & 0.086 \\
\hline & $\begin{array}{l}\text { Task Condition * } \\
\text { Stimulation }{ }^{*} \\
\text { Frequency }\end{array}$ & 1.855 & 5 & 0.104 \\
\hline & $\begin{array}{l}\text { Task Condition * } \\
\text { Stimulation }{ }^{*} \\
\text { Frequency * Age }\end{array}$ & 0.815 & 20 & 0.540 \\
\hline & $\begin{array}{l}\text { Task Condition }{ }^{\star} \\
\text { Frequency }{ }^{\star} \text { Age } \\
\text { Group }\end{array}$ & 0.927 & 20 & 0.465 \\
\hline
\end{tabular}


Figure 1: Task description and experimental design. A bimanual tracking task (BTT) was designed which involves the use of both hands to rotate a dial at different frequency ratios in order to follow a moving target on a line. An iso-frequency condition of 1:1 indicates that both left and right hand must rotate at the same relative frequency in order to accurately follow the moving target. Ratios of 3:1 and 2:1 indicate greater involvement of the left hand compared to the right hand, and vice versa with conditions 1:2 and 1:3. Quadrant position of the task determines whether the relationship between hands is in-phase or anti-phase. B) Subjects were seated in a comfortable position with their hands covered by a bench. For experiment 2, anodal tDCS was delivered using a sponge electrode secured to the position of the right motor cortex by rubber straps. C) The design of the study. Experiment 1 consisted of two groups of participants (young and elder) who performed the BTT during concurrent EEG recording. In experiment 2, a separate group of young and elder participants was recruited, and participants attended two sessions in a balanced cross-over design where anodal or sham tDCS was administered.

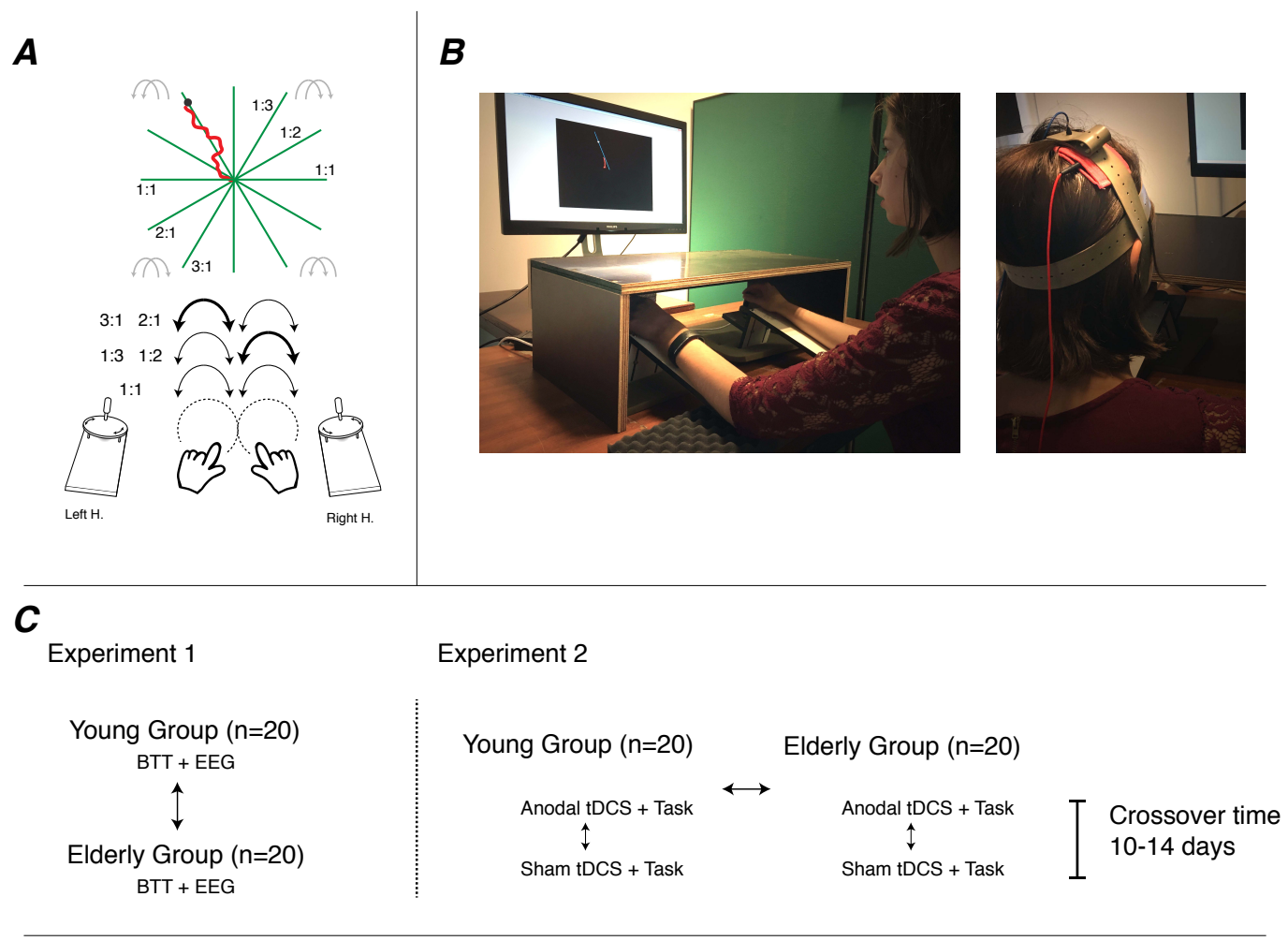


Figure 2: Task performance on the BTT from Experiment 1. Task accuracy was computed as a function of the displacement between the subject's position and the track and target position. Thus, a lower value indicates better performance. Over all conditions, younger participants performed with better accuracy than elderly (all values of $\mathrm{P}<0.05)$. The iso-frequency condition $(1: 1)$ was found to be the easiest in both groups, while the left-hand dominant conditions (2:1 and 3:1) were the most difficult to perform.

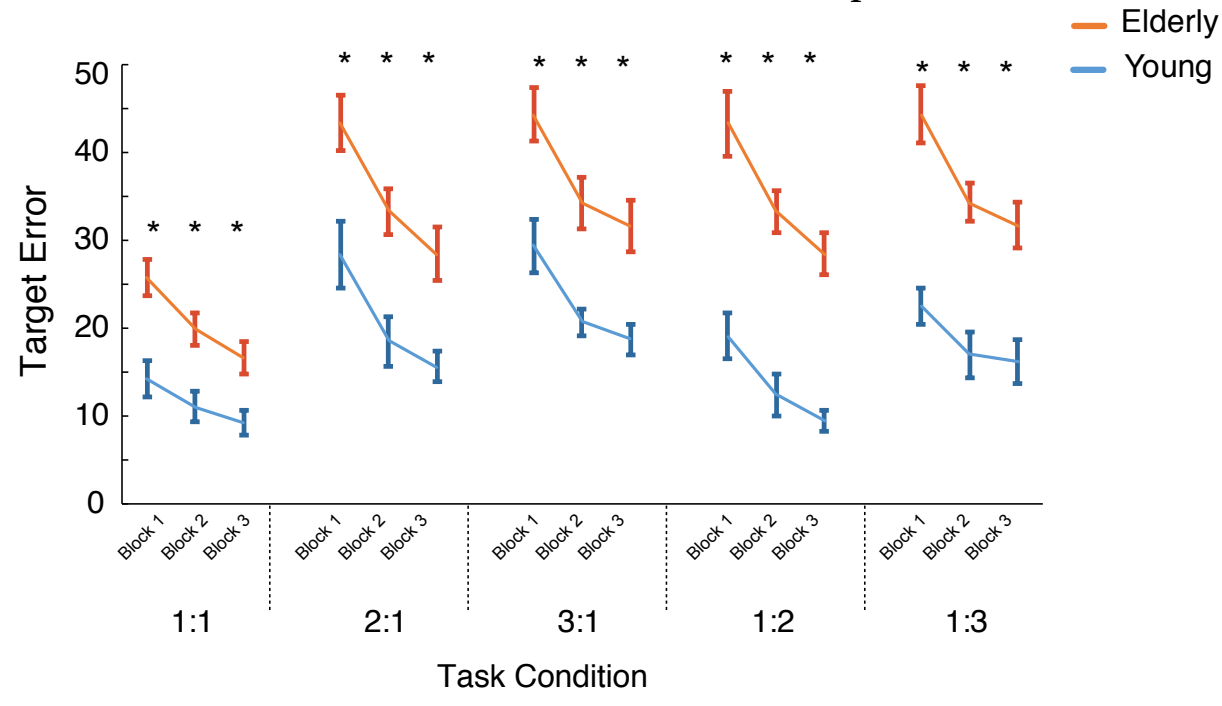


Figure 3: EEG connectivity differences between the young and elderly. Functional connectivity was computed as the weighted phase lag index between all 32 pairwise EEG channels. This measure was computed over different frequency bands and the average connectivity over all channels was compared between the two groups (young vs elderly). A-B) Gamma-frequency connectivity tended to be higher in younger participants, while connectivity in frequencies theta and alpha was higher in the elderly. C) To explore if the higher gamma frequency connectivity in the young age group led to better performance for each condition of the task, pairwise channel connectivity in each subject's mean WPLI matrix was regressed to the subject's trial performance in order to highlight the regions where connectivity correlated strongly with performance. Regions in which the regression analysis showed a significant correlation $(\mathrm{P}<0.05)$ are shown as solid lines, where darker orange/red lines indicate smaller values of $\mathrm{p}$ in the multiple regression. D) Accurate performance in task conditions where the left hand required greater involvement was found to be correlated with greater connectivity between right motor and ipsilateral parietal areas.

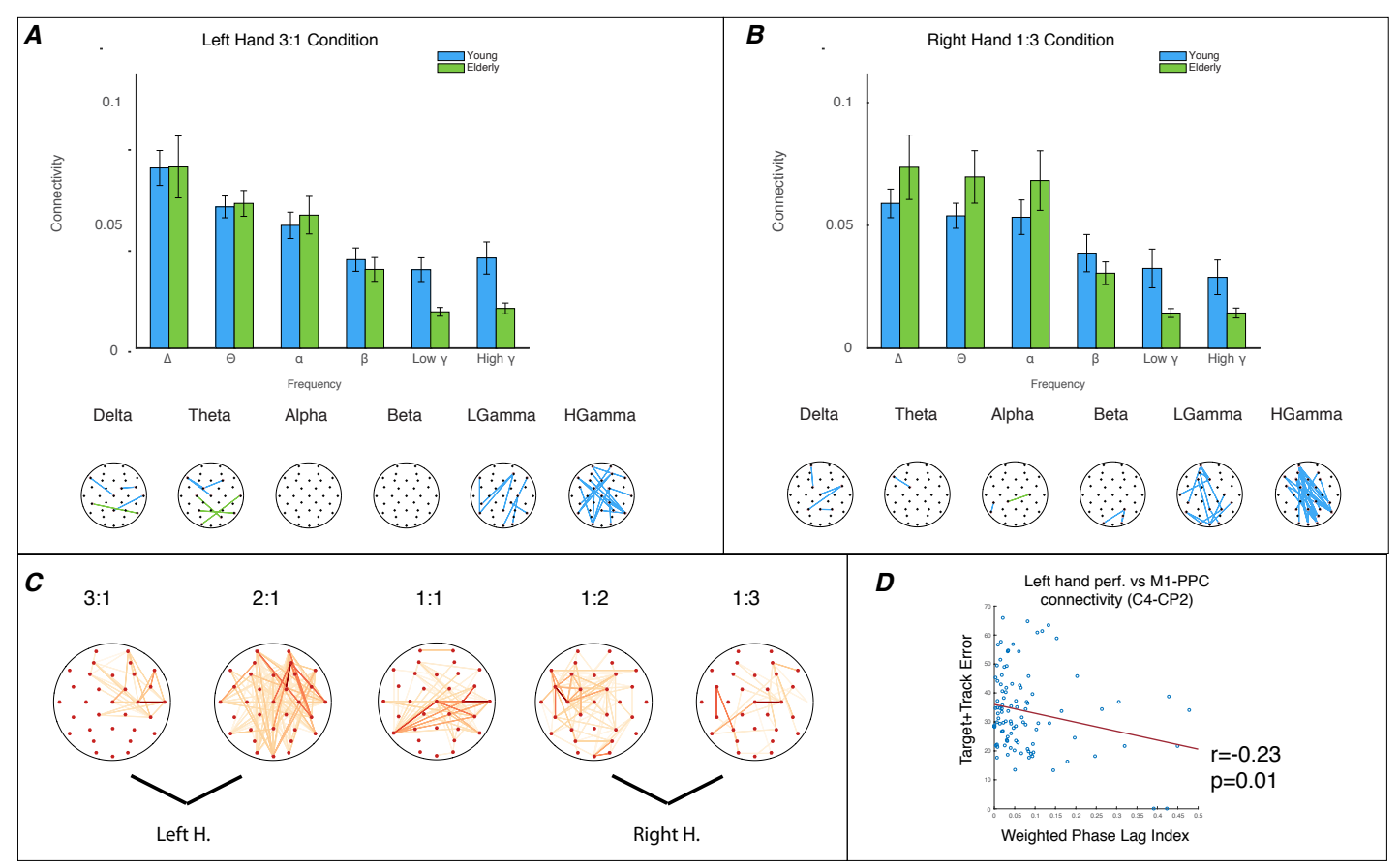


Figure 4: Task performance on the BTT following tDCS. A) Task performance, measured as the displacement error between the subject's cursor and the track and target was compared between age groups and stimulation conditions. Similar to findings of experiment 1 (Figure 2), younger participants performed more accurately than elderly in all tasks, and performance generally improved over the course of the session. Stimulation tended to result in less error over all task conditions for both age groups, and was significant in reducing the error of the left hand 2:1 task in the first block ( $\mathrm{p}=0.03$, uncorrected). B) Task performance in the final block, during which no visual feedback was provided. For the elderly, stimulation effectively enhanced the performance in the left hand 3:1 block. Other conditions also tended to show general improvement in performance with $\mathrm{tDCS}$.

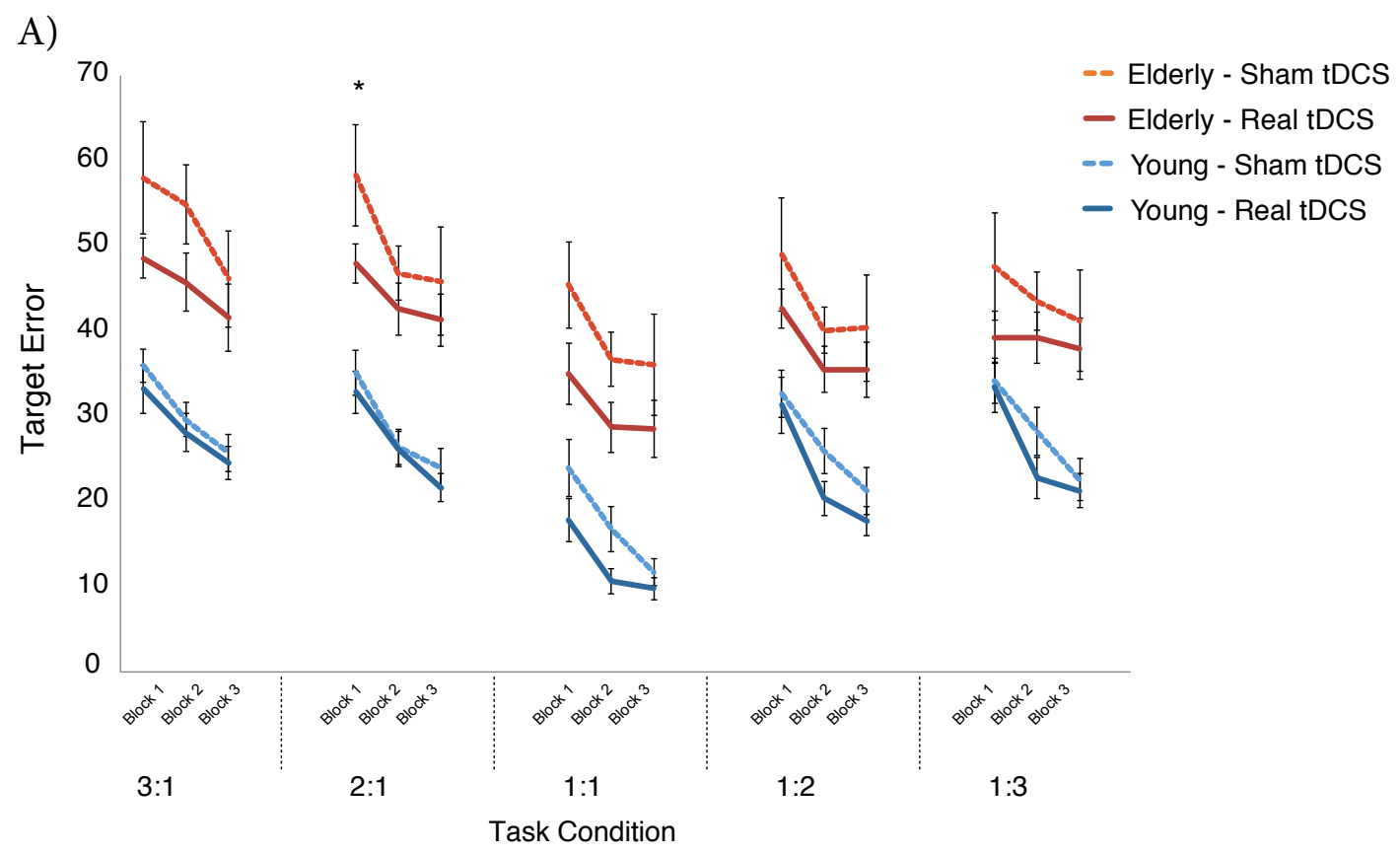

B)

\section{Post-performance evaluation \\ (no feedback)}

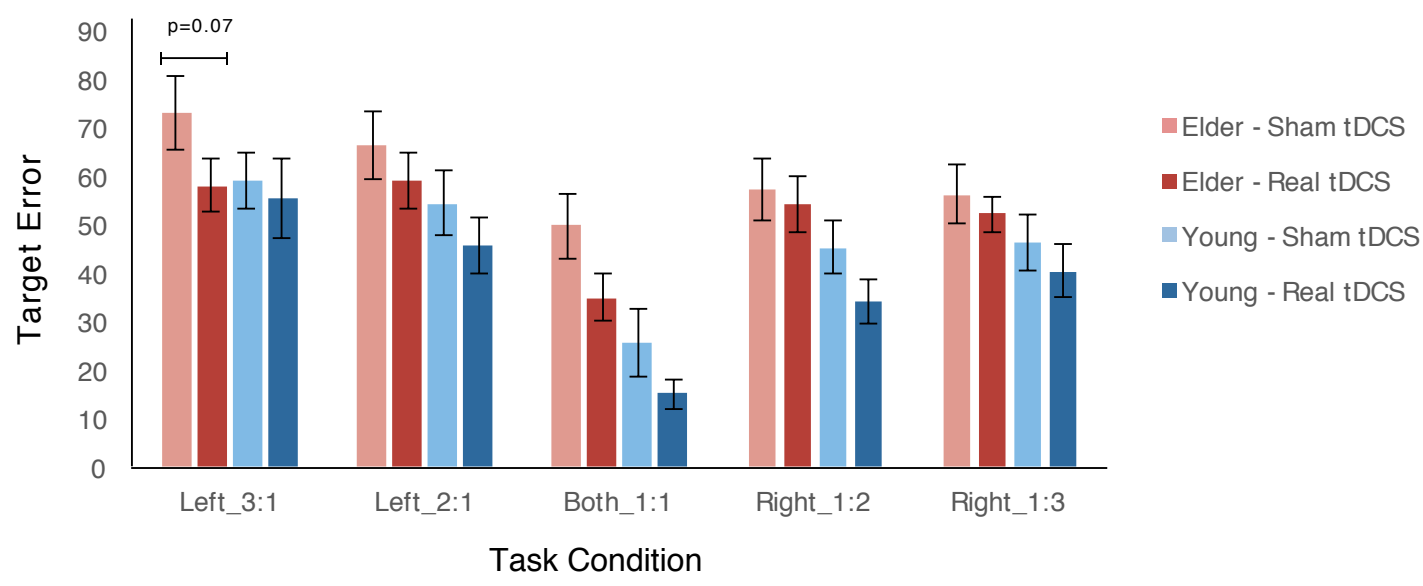


Figure 5: Age- and task-dependent alterations in functional connectivity between stimulation conditions. For each hand condition (left hand: 3:1, 2:1; right hand: 1:3,1:2), the weighted phase lag index (WPLI) connectivity over all channels was grand-averaged, and compared between the real and sham tDCS sessions. Spatial extent of these differences were explored by means of paired contrasts (two-tailed t-tests, $\mathrm{p}<0.05$ FDR corrected; red colors indicate tDCSinduced increase while blue indicates connectivity decrease). A) Connectivity differences between real and sham tDCS in younger participants. In trial conditions requiring heavier usage of the left hand, tDCS induced a small increase in lower frequency delta band, whereas connectivity in other frequencies shifted in the direction of decrease. No overall significant differences were observed in the right hand task conditions. B) In the elderly participants, for the left hand trials, stimulation induced an increase in connectivity in the low and high gamma frequency band, however no overall difference was statistically significant. When observing the topology of the contrast, a clear trend towards increasing connectivity at higher frequency bands becomes apparent, and these increases appear concentrated in the right frontal regions. The overall connectivity in right hand-related trials was not affected by tDCS, with the exception of the delta band.

\section{$\boldsymbol{A}$}

Young Group
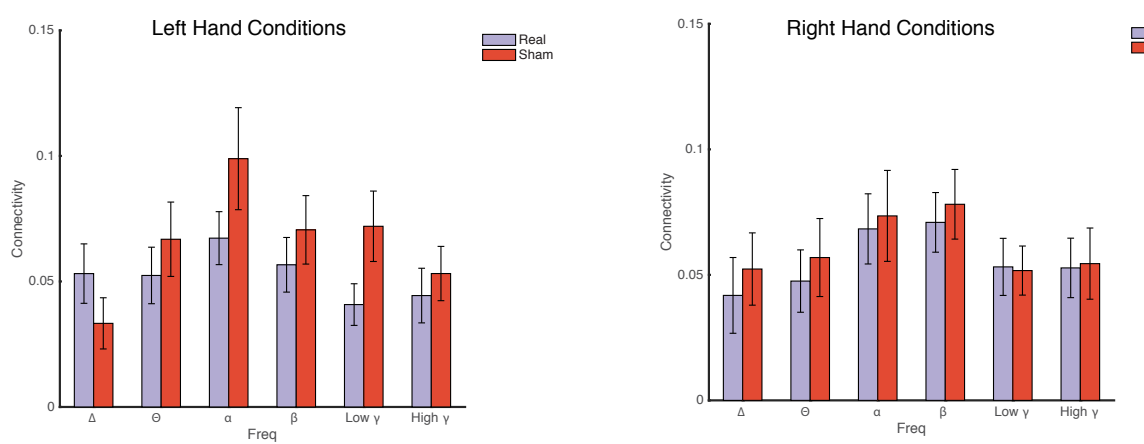

Contrast: [Real - Sham]
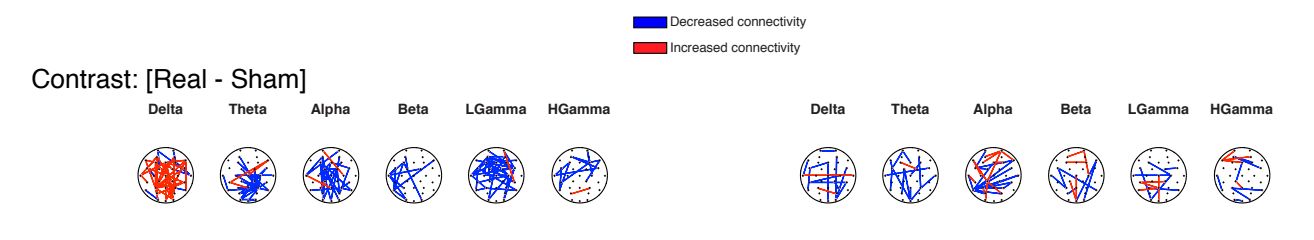

$B$

Elderly Group
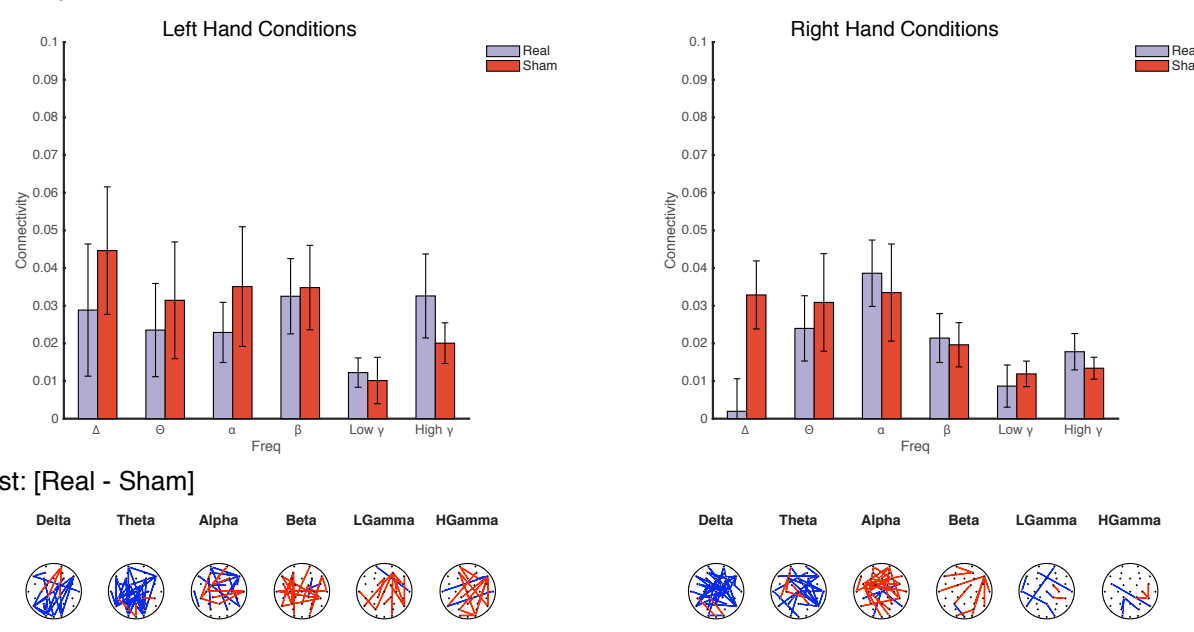


\section{References}

Amazeen EL, Amazeen PG, Treffner PJ \& Turvey MT (1997). Attention and handedness in bimanual coordination dynamics. J Exp Psychol Hum Percept Perform 23, 1552-1560.

Beets IAM, Gooijers J, Boisgontier MP, Pauwels L, Coxon JP, Wittenberg G \& Swinnen SP (2015). Reduced Neural Differentiation Between Feedback Conditions After Bimanual Coordination Training with and without Augmented Visual Feedback. Cereb Cortex 25, 1958-1969.

Benjamini Y \& Hochberg Y (1995). Controlling the false discovery rate: a practical and powerful approach to multiple testing. J $R$ Stat Soc B 57, 289-300. Available at: http://www.stat.purdue.edu/ doerge/BIOINFORM.D/FALL06/Benjamin $\mathrm{i}$ and $\mathrm{Y}$ FDR.pdf\%5Cnhttp://engr.case.edu/ray_soumya/mlrg/controlling_fdr_be njamini95.pdf.

Bennett IJ \& Madden DJ (2014). Disconnected aging: Cerebral white matter integrity and age-related differences in cognition. Neuroscience 276, 187205.

Boggio PS, Castro LO, Savagim EA, Braite R, Cruz VC, Rocha RR, Rigonatti SP, Silva MTA \& Fregni F (2006). Enhancement of non-dominant hand motor function by anodal transcranial direct current stimulation. Neurosci Lett 404, 232-236.

Burke SN \& Barnes CA (2006). Neural plasticity in the ageing brain. Nat Rev Neurosci 7, 30-40.

Cabeza R, Anderson ND, Locantore JK \& McIntosh AR (2002). Aging gracefully: compensatory brain activity in high-performing older adults. Neuroimage 17, 1394-1402.

Celsis P (2000). Age-related cognitive decline, mild cognitive impairment or preclinical Alzheimer's disease? Ann Med 32, 6-14.

Fornito A, Zalesky A \& Breakspear M (2013). Graph analysis of the human connectome: Promise, progress, and pitfalls. Neuroimage 80, 426-444.

Franz EA, Rowse A \& Ballantine B (2002). Does Handedness Determine Which Hand Leads in a Bimanual Task? J Mot Behav 34, 402-412.

Fujiyama H, Hyde J, Hinder MR, Kim SJ, McCormack GH, Vickers JC \& Summers JJ (2014). Delayed plastic responses to anodal tDCS in older adults. Front Aging Neurosci 6, 1-9.

Fujiyama H, Van Soom J, Rens G, Gooijers J, Leunissen I, Levin O \& Swinnen SP (2016). Age-Related Changes in Frontal Network Structural and Functional Connectivity in Relation to Bimanual Movement Control. J Neurosci 36, 1808-1822.

Furuya S, Nitsche MA, Paulus W \& Altenmüller E (2013). Early optimization in finger dexterity of skilled pianists: implication of transcranial stimulation. BMC Neurosci 14, 35.

Ginestet CE, Nichols TE, Bullmore ET \& Simmons A (2011). Brain Network Analysis : Separating Cost from Topology Using Cost-Integration. ; DOI: 10.1371/journal.pone.0021570. 
Ginestet CE \& Simmons A (2011). Statistical parametric network analysis of functional connectivity dynamics during a working memory task. Neuroimage 55, 688-704.

Harms KJ, Rioult-Pedotti MS, Carter DR \& Dunaevsky A (2008). Transient Spine Expansion and Learning-Induced Plasticity in Layer 1 Primary Motor Cortex. J Neurosci 28, 5686-5690.

Kannurpatti SS, Motes MA, Rypma B \& Biswal BB (2010). Neural and vascular variability and the fMRI-BOLD response in normal aging. Magn Reson Imaging 28, 466-476.

Kannus P, Parkkari J, Niemi S \& Palvanen M (2005). Fall-Induced Deaths Among Elderly People. Am J Public Health 95, 422-424.

Kennerley SW, Diedrichsen J, Hazeltine E, Semjen A \& Ivry RB (2002). Callosotomy patients exhibit temporal uncoupling during continuous bimanual movements. Nat Neurosci 5, 376-381.

Logsdon RG, Gibbons LE, McCurry SM \& Teri L (2002). Assessing quality of life in older adults with cognitive impairment. Psychosom Med 64, 510519.

Lustig C, Shah P, Seidler R \& Reuter-Lorenz PA (2009). Aging, training, and the brain: a review and future directions. Neuropsychol Rev 19, 504-522.

Murphy K \& Peters M (1994). Right-handers and left-handers show differences and important similarities in task integration when performing manual and vocal tasks concurrently. Neuropsychologia 32, 663-674.

Nitsche MA, Cohen LG, Wassermann EM, Priori A, Lang N, Antal A, Paulus W, Hummel F, Boggio PS, Fregni F \& Pascual-Leone A (2008). Transcranial direct current stimulation: State of the art 2008. Brain Stimul 1, 206-223.

Nitsche MA \& Paulus W (2000). Excitability changes induced in the human motor cortex by weak transcranial direct current stimulation. J Physiol 527 Pt 3, 633-639.

Nitsche MA \& Paulus W (2001). Sustained excitability elevations induced by transcranial DC motor cortex stimulation in humans Sustained excitability elevations induced by transcranial DC motor cortex stimulation in. Neurology 57, 1899-1901.

Nitsche MA, Schauenburg A, Lang N, Liebetanz D, Exner C, Paulus W \& Tergau F (2003). Facilitation of Implicit Motor Learning by Weak Transcranial Direct Current Stimulation of the Primary Motor Cortex in the Human. J Cogn Neurosci 15, 619-626.

ONU (2015). World Population Prospects 2015 - Data Booklet (ST/ESA/SER.A/377). 20.

Pascual-Leone A, Valls-sole J, Wassermann EM \& Hallett M (1994). Responses to rapid-rate transcranial magnetic stimulation of the human motor cortex. Brain 117, 847-858.

Pellicciari MC, Brignani D \& Miniussi C (2013). Excitability modulation of the motor system induced by transcranial direct current stimulation: A multimodal approach. Neuroimage 83, 569-580. 
Perceval G, Flöel A \& Meinzer M (2016). Can transcranial direct current stimulation counteract age-associated functional impairment? Neurosci Biobehav Rev 65, 157-172.

Polanía R, Nitsche MA \& Paulus W (2011). Modulating functional connectivity patterns and topological functional organization of the human brain with transcranial direct current stimulation. Hum Brain Mapp 32, 1236-1249.

Reis J, Schambra HM, Cohen LG, Buch ER, Fritsch B, Zarahn E, Celnik PA \& Krakauer JW (2009). Noninvasive cortical stimulation enhances motor skill acquisition over multiple days through an effect on consolidation. Proc Natl Acad Sci U S A 106, 1590-1595.

Resnick SM, Pham DL, Kraut MA, Zonderman AB \& Davatzikos C (2003). Longitudinal Magnetic Resonance Imaging Studies of Older Adults: A Shrinking Brain. J Neurosci 23, 3295-3301.

Richiardi J, Eryilmaz H, Schwartz S, Vuilleumier P \& Van De Ville D (2011). Decoding brain states from fMRI connectivity graphs. Neuroimage 56, 616-626.

Ridderikhoff A (2005). Unraveling Interlimb Interactions Underlying Bimanual Coordination. J Neurophysiol 94, 3112-3125.

Salat DH, Buckner RL, Snyder AZ, Greve DN, Desikan RSR, Busa E, Morris JC, Dale AM \& Fischl B (2004). Thinning of the cerebral cortex in aging. Cereb Cortex 14, 721-730.

Scherder E, Dekker W \& Eggermont L (2008). Higher-Level Hand Motor Function in Aging and (Preclinical) Dementia: Its Relationship with (Instrumental) Activities of Daily Life - A Mini-Review. Gerontology 54, 333-341.

Seidler RD, Bernard JA, Burutolu TB, Fling BW, Gordon MT, Gwin JT, Kwak Y \& Lipps DB (2010). Motor control and aging: Links to age-related brain structural, functional, and biochemical effects. Neurosci Biobehav Rev 34, 721-733.

Serbruyns L, Gooijers J, Caeyenberghs K, Meesen RL, Cuypers K, Sisti HM, Leemans a. \& Swinnen SP (2013). Bimanual motor deficits in older adults predicted by diffusion tensor imaging metrics of corpus callosum subregions. Brain Struct Funct1-18.

Sisti HM, Geurts M, Clerckx R, Gooijers J, Coxon JP, Heitger MH, Caeyenberghs K, Beets IAM, Serbruyns L \& Swinnen SP (2011). Testing multiple coordination constraints with a novel bimanual visuomotor task. PLoS One; DOI: 10.1371/journal.pone.0023619.

Spencer R \& Ivry R (2005). Bimanual coordination during rhythmic movements in the absence of somatosensory feedback. J ...2901-2910.

Spirduso WW, Francis KL \& MacRae PG (2005). Physical dimensions of aging. Human Kinetics.

Stagg CJ, Bestmann S, Constantinescu AO, Moreno LM, Allman C, Mekle R, Woolrich M, Near J, Johansen-Berg H, Rothwell JC, Moreno Moreno L, Allman C, Mekle R, Woolrich M, Near J, Johansen-Berg H \& Rothwell JC (2011). Relationship between physiological measures of excitability and levels of glutamate and GABA in the human motor cortex. J Physiol 589, $5845-5855$. 
Stefan K, Kunesch E, Cohen LG, Benecke R \& Classen J (2000). Induction of plasticity in the human motor cortex by paired associative stimulation. Brain 123 Pt 3, 572-584.

Swinnen SP \& Wenderoth N (2004). Two hands, one brain: Cognitive neuroscience of bimanual skill. Trends Cogn Sci 8, 18-25.

Vinck M, Oostenveld R, van Wingerden M, Battaglia F \& Pennartz CMA (2011). An improved index of phase-synchronization for electrophysiological data in the presence of volume-conduction, noise and sample-size bias. Neuroimage 55, 1548-1565.

Vines BW, Nair DG \& Schlaug G (2006). Contralateral and ipsilateral motor effects after transcranial direct current stimulation. Neuroreport 17, 671674.

Xu T, Yu X, Perlik AJ, Tobin WF, Zweig JA, Tennant K, Jones T \& Zuo Y (2009). Rapid formation and selective stabilization of synapses for enduring motor memories. Nature 462, 915-919.

Zimerman M \& Hummel FC (2010). Non-Invasive Brain Stimulation: Enhancing Motor and Cognitive Functions In Healthy Old Subjects. Front Aging Neurosci 2, 1-12. 


\section{Summary \& concluding remarks}

In this final section, we summarize the main findings of the conducted experiments before moving on to discuss their significance to the field. At the end, we will provide an outlook, and suggestions for future research directions.

\section{Overview of results}

Study 1: Efficacy of Anodal Transcranial Direct Current Stimulation is Related to Sensitivity to Transcranial Magnetic Stimulation

Research question: Does individual susceptibility to non-invasive stimulation protocols predict the efficacy of plasticity induction at an individual level.

Methods: Using a median-split procedure, 34 participants who received tDCS were divided into subgroups based on their TMS stimulus intensity to obtain a $1 \mathrm{mV}$ amplitude (SI1mV). As a control, 36 participants who received PAS were also divided into subgroups. Poststimulation measurements were grand-averaged within two time windows: Early $(0-30 \mathrm{~min})$ and Late $(60-120 \mathrm{~min})$. Separate ANOVAs were performed for each conditioning protocol, with one between-subject factor (Group: Low Intensity vs. High Intensity) and a within-subject repeating factor (Time: Early vs. Late epoch).

Results: MEPs were enhanced following anodal tDCS. This effect was larger in participants more sensitive to TMS (i.e., those participants who required a lower TMS SI $1 \mathrm{mV}$ intensity), as compared to those less sensitive to TMS. No relationship was observed for cathodal tDCS, or inhibitory or excitatory PAS.

Conclusion: Accounting for variation in individual sensitivity to non-invasive baseline stimulation may enhance the utility of tDCS as a tool for understanding brain-behavior interactions and a method for clinical interventions.

Study 2: Systematic evaluation of the impact of stimulation intensity on neuroplastic after-effects induced by transcranial direct current stimulation

Research question: What is the relationship between tDCS intensity and excitability after-effects, and does this relationship interact with any individual factors?

Methods: Two groups of subjects (anodal, cathodal) received five intensities of tDCS (sham, 0.5, 1.0, 1.5 and $2.0 \mathrm{~mA}$ ) over separate sessions, and motor cortical excitability was assessed using TMS for up to $2 \mathrm{~h}$.

Results: With anodal tDCS, all active intensities resulted in equivalent facilitatory effects relative to sham while for cathodal tDCS, only $1.0 \mathrm{~mA}$ resulted in sustained excitability diminution. Furthermore, TMS SI1mV (stimulus intensity for $1 \mathrm{mV}$ MEP amplitude) sensitivity correlated negatively with $1.0 \mathrm{~mA}$ anodal tDCS effects on excitability, in accordance with our findings from study 1. 
Conclusion: Anodal tDCS with intensities between $0.5 \mathrm{~mA}$ to $2.0 \mathrm{~mA}$ reliably induce cortical excitability enhancement, with no specific intensity being more or less optimal, whereas an intensity around $1.0 \mathrm{~mA}$ is optimal for excitability diminution with cathodal tDCS.

Study 3: Current intensity- and polarity-dependent effects of transcranial direct current stimulation on cortical activation: an fMRI study

Research question: What is the relationship between tDCS intensity and the resulting hemodynamic effects.

Methods: Two groups of subjects (anodal, cathodal) received five intensities of tDCS (sham, 0.5, 1.0, 1.5 and $2.0 \mathrm{~mA}$ ) over separate sessions, and cerebral blood flow (CBF) was assessed using resting-state ASL MRI for up to $2 \mathrm{~h}$ following stimulation. Time-course change in rCBF was measured as the mean cortical perfusion at 10 timepoints (pre-tDCS, during and up to $2 \mathrm{~h}$ following stimulation), and analyzed among four regions of interest, including the left M1, right $\mathrm{M} 1$, right frontal superior orbit, and a control region in the right superior temporal gyrus. As an additional assessment, regionwise cortico-cortical connectivity of left M1 was evaluated by means of a seed-based functional connectivity analysis.

Results: All active intensities of anodal tDCS led to increased left M1 perfusion, where $2.0 \mathrm{~mA}$ tDCS resulted in the greatest increase when compared with sham. Cathodal tDCS over all active intensities led to a relatively modest decrease in left M1 perfusion from baseline, yet only $2.0 \mathrm{~mA}$ tDCS showed a significant decrease compared to sham.

Conclusion: These findings provide, for the first time, evidence of lasting tDCS-induced alterations in arterial perfusion in the cerebral vascular system, which are linearly coupled to tDCS parameters in a polarity- and intensity-dependent relationship.

Study 4: Investigating bimanual motor coordination in healthy young and older adults using EEG and transcranial direct current stimulation (tDCS)

Research question: What are the kinematic and physiological markers of agerelated differences during acquisition of complex bimanual motor control tasks. Can performance of bimanual motor skills be improved in the elderly using tDCS.

Methods: Two experiments were conducted. First continuous EEG was recorded while young and elderly subjects performed the bimanual tracking task (BTT). In a second experiment, right $\mathrm{M} 1$ anodal tDCS $(1.0 \mathrm{~mA}, 20 \mathrm{~min})$ was applied during the first and second block, which was then followed by an EEG recording in the third block.

Results: Task performance in younger subjects was more accurate than in elderly. Younger subjects exhibited significantly stronger functional connectivity in the theta power band, which was also a reliable predictor for accurate performance of the task. For the 
second experiment, we found that real tDCS caused significant performance improvements in the elderly but not in the young.

Conclusion: We show that both functional connectivity and inter-limb kinematics underlying bimanual motor coordination are different between the young and elderly. We further show that a single session of tDCS applied to the motor cortex was able to significantly improve the bimanual performance in both young and elderly. Although further studies are needed to optimize tDCS parameters for enhanced and prolonged effects, this non-invasive stimulation technique may be a viable tool in restituting and even further optimizing the learning of complex motor functions in the aging population.

\section{Discussion}

The studies included in this thesis address issues which are important for the further understanding of the mechanisms underlying neuroplasticity, as well as in guiding future optimizations for enhancing efficacy of tDCS. Our first discovery that an intrinsic individual factor, baseline sensitivity to TMS, may show some predisposition to the extent of tDCS efficacy is a unique and important finding as it implies that tDCS protocols may need to be individually adjusted and optimized to the subject, in order to achieve the most beneficial results. This is also important, because as shown in our second study, stimulation intensities that are too high may not necessarily yield correspondingly greater effects, as is shown in the case of cathodal tDCS. Morever, the findings from our first study on TMS excitability were replicated in our second study, and when taken together with findings from our third (MRI) study, where we observed a small impact of gray matter volume in affecting tDCS response, lends to the need for integrating more anatomical and physiological factors into multi-level computational models of tDCS (Ruffini et al.2013). Future studies in the field should consider the use of such computationally guided models and report physiological effects when available in order for providing validation and continuing their optimization. The second and third study address fundamental questions about the relationship between the current intensity parameter and two physiological outcomes - cortical excitability, which can be measured using TMS, and cerebral blood flow, which can be measured using ASL, or indirectly using BOLD fMRI. With regard to cortical excitability alterations, in principle accordance to previous studies (Bastani \& Jaberzadeh, 2013; Kidgell et al., 2013), we did not observe a differential effect of anodal tDCS, as no single intensity showed significantly better or worse effect than another, either in the amplitude of excitability increase, or the duration of the effect. With cathodal tDCS, we observed a non-linear effect where $1.0 \mathrm{~mA}$ showed a strong stable reduction in excitability diminution lasting up to $90 \mathrm{~min}$ whereas other intensities did not yield a reliable effect. These findings, as well as a previous study in which cathodal tDCS was performed on adolescents (Moliadze et al., 2015), add further support to the bidirectional role of calcium ion flux in triggering LTD, LTP or neither (so called "no man's land") in the 
glutamatergic synapse (Cho et al., 2001; Lisman, 2001). With regard to cerebral blood flow, for anodal tDCS, we observed a linear effect of DC intensity on $\mathrm{rCBF}$, in which $2.0 \mathrm{~mA}$ showed the strongest increase over the longest time span $(2 \mathrm{~h})$. Cathodal tDCS resulted in generally weak reductions in $\mathrm{rCBF}$, yet nevertheless, $2.0 \mathrm{~mA}$ produced a significant decrease in $\mathrm{rCBF}$ also spanning the entire $2 \mathrm{~h}$ duration of the measurement. Besides our aim to understand the relationship between tDCS intensity and the respective physiological responses, we were also secondarily interested to assess the extent of the relationship between the two physiological experiments (i.e., intensity-wise alterations of cortical excitability on the one hand, and intensity-wise effects of cereberal blood flow on the other). As both experiments made use of the same participants, we computed a group-average correlation in the time course of the response, which is presented in Figure 1. Based on this correlation, we found that 1.0, 1.5 and $2.0 \mathrm{~mA}$ anodal tDCS and $1.0 \mathrm{~mA}$ cathodal tDCS correlated strongly between $\mathrm{rCBF}$ and excitability alterations. The relatively strong coupling in these findings (at least for anodal tDCS, where effects were more pronounced) indicate that functional imaging may be a valid and useful tool to transfer previous findings of tDCS-induced neuroplasticity modulations in the motor cortex to other cortical areas where TMS measurements may be difficult to obtain. In a final fourth study, we explored the functional relevance of tDCS in enhancing bimanual motor coordination in the aging population. Our promising findings here indicate that tDCS is not just limited to enhanced motor excitability, but also well suited for relevantly enhancing the learning of complex movement patterns, which is useful for the daily life quality in the elderly. Further studies in this line could consider additional tDCS parameters, such as multiple sessions of tDCS with short repetition intervals which might be effective in inducing latephase plasticity (Monte-Silva et al., 2013).

As the popularity of tDCS continues to grow and broaden in both basic and applied research, more work needs to be done to progress in the avenue of optimization. First, in the domain of physiological research, further investigations on the effect of methodological parameters are still required. Particularly, investigating stimulation intensities higher than $2.0 \mathrm{~mA}$ might be useful to supplant the findings of our work here. In this aspect, developing new methods for adequate investigator and participant blinding may be useful to circumvent perception of high intensities (O'Connell et al. 2012). A second objective should be to obtain additional neurophysiological measures that are sensitive to neuromodulatory interventions, and also relevant for transferability to applied domains. These measures should ideally be able to reflect physiological activity from a variety of cortical sources, taking advantage of neuroimaging methods such as EEG, functional near-infrared spectroscopy (fNIRS), or fMRI. Subsequently, these metrics can be simulated within computational models, in order to predict the effect of stimulation protocols (Bestmann, 2015). Validation and feedback to these models is also crucial, and could be the objective in applied settings, such as with cognitive paradigms in young healthy cohorts, or in multi-center clinical settings for use in neurological or psychiatric treatment (Flöel, 2014). If successful, this 
multilevel research framework could one day offer the opportunity for "closed-loop" protocols, where the stimulation device adapts itself to the subject's ongoing physiological activity in order to induce highly selective neuroplastic modulations.

Anodal tDCS

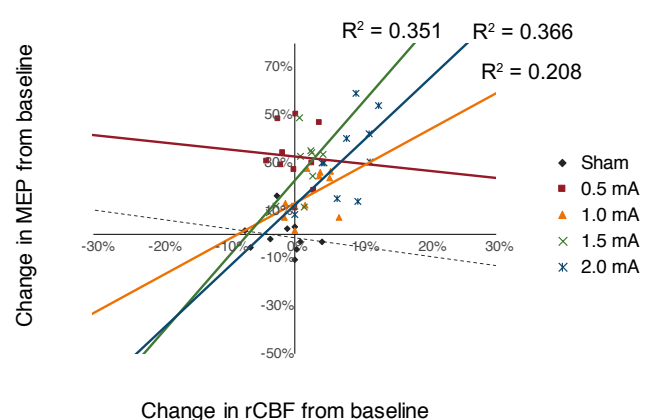

Cathodal tDCS

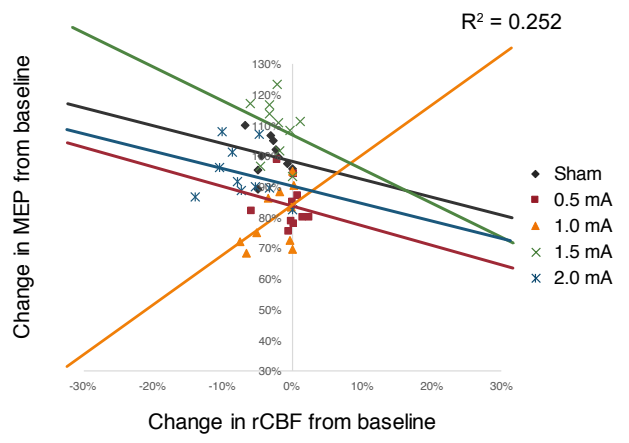

Figure 1. Correlation in time-course averages between anodal and cathodal tDCS alterations on cortical excitability ( $y$-axis) and effects on cerebral blood flow (x-axis). Positive correlations were observed in anodal tDCS intensities greater than $0.5 \mathrm{~mA}$, and was highest for $2.0 \mathrm{~mA}$. For cathodal tDCS, most intensities revealed an inverse relationship with the exception of $1.0 \mathrm{~mA}$, which showed a positive correlation. This is likely due to the fact that $1.0 \mathrm{~mA}$ cathodal tDCS was found to be the optimal intensity in decreasing cortical excitability (Jamil et al., 2016). 


\section{References}

Andersen P \& Lomo T (1966). Mode of activation of hippocampal pyramidal cells by excitatory synapses on dendrites. Exp Brain Res 2, 247-260.

Bassett DS \& Bullmore E (2006). Small-World Brain Networks. Neurosci 12, $512-523$.

Bastani A \& Jaberzadeh S (2013). Differential Modulation of Corticospinal Excitability by Different Current Densities of Anodal Transcranial Direct Current Stimulation. PLoS One 8, 1-8.

Batsikadze G, Moliadze V, Paulus W, Kuo M-F \& Nitsche MA (2013). Partially non-linear stimulation intensity-dependent effects of direct current stimulation on motor cortex excitability in humans. J Physiol 591, 19872000.

Beck H, Goussakov I V., Lie A, Helmstaedter C \& Elger CE (2000). Synaptic Plasticity in the Human Dentate Gyrus. J Neurosci.

Bindman LJ, Lippold OCJ \& Redfearn JWT (1964). The action of brief polarizing currents on the cerebral cortex of the rat (1) during current flow and (2) in the production of long-lasting after-effects. J Physiol 172, $369-382$.

Bliss TVP \& Lomo T (1973). Long-lasting potentiation of synaptic transmission in the dentate area of the anaesthetized rabbit following stimulation of the preforant path. J Physiol 232, 331-356.

Boggio P, Khoury L, Martins D, Martins O, de Macedo E \& Fregni F (2009). Temporal cortex direct current stimulation enhances performance on a visual recognition memory task in Alzheimer disease. J Neurol Neurosurg Psychiatry 80, 444-4447.

Brunoni AR, Valiengo L, Baccaro A, Zanão T a, de Oliveira JF, Goulart A, Boggio PS, Lotufo P a, Benseñor IM \& Fregni F (2013). The sertraline vs. electrical current therapy for treating depression clinical study: results from a factorial, randomized, controlled trial. JAMA psychiatry 70, 383391.

Caillard O, Ben-Ari Y \& Gaiarsa JL (1999). Long-term potentiation of GABAergic synaptic transmission in neonatal rat hippocampus. J Physiol 518 ( Pt 1, 109-119.

Chen R \& Rothwell JC (2012). Cortical connectivity: Brain stimulation for assessing and modulating cortical connectivity and function ed. Chen $\mathrm{R}$ \& Rothwell JC. Cortical Connect Brain Stimul Assess Modul Cortical Connect Funct1-365.

Chen WR, Lee S, Kato K, Spencer DD, Shepherd GM \& Williamson A (1996). Long-term modifications of synaptic efficacy in the human inferior and middle temporal cortex. Proc Natl Acad Sci U S A 93, 8011-8015.

Chew T, Ho K-A \& Loo CK (2015). Inter- and intra-individual variability in response to transcranial direct current stimulation (tDCS) at varying current intensities. Brain Stimul1-8.

Cho K, Aggleton JP, Brown MW \& Bashir ZI (2001). An experimental test of the role of postsynaptic calcium levels in determining synaptic strength using perirhinal cortex of rat. J Physiol 532, 459-466. 
Classen J, Liepert J, Wise SP, Hallett M \& Cohen LG (1998). Rapid plasticity of human cortical movement representation induced by practice. $J$ Neurophysiol 79, 1117-1123.

Coan EJ \& Collingridge GL (1987). Characterization of an N-methyl-Daspartate receptor component of synaptic transmission in rat hippocampal slices. Neuroscience 22, 1-8.

Cooke SF \& Bliss TVP (2006a). Plasticity in the human central nervous system. Brain 129, 1659-1673.

Cooke SF \& Bliss TVP (2006b). Plasticity in the human central nervous system. Brain 129, 1659-1673.

Cummings JA, Mulkey RM, Nicoll RA \& Malenka RC (1996). Ca2+ signaling requirements for long-term depression in the hippocampus. Neuron 16, 825-833.

Detre JA \& Alsop DC (1999). Perfusion magnetic resonance imaging with continuous arterial spin labeling: Methods and clinical applications in the central nervous system. Eur J Radiol 30, 115-124.

Fitzgerald PB, Fountain S \& Daskalakis ZJ (2006). A comprehensive review of the effects of rTMS on motor cortical excitability and inhibition. Clin Neurophysiol 117, 2584-2596.

Friauf E, Fischer AU \& Fuhr MF (2015). Synaptic plasticity in the auditory system: a review. Cell Tissue Res 361, 177-213.

Hess G \& Donoghue JP (1994). Long-term potentiation of horizontal connections provides a mechanism to reorganize cortical motor maps. $J$ Neurophysiol 71, 2543-2547.

Heynen a J \& Bear MF (2001). Long-term potentiation of thalamocortical transmission in the adult visual cortex in vivo. J Neurosci 21, 9801-9813.

Islam N, Aftabuddin M, Moriwaki A, Hattori Y \& Hori Y (1995a). Increase in the calcium level following anodal polarization in the rat brain. Brain Res 684, 206-208.

Islam N, Moriwaki A, Hattori Y, Hayashi Y, Lu Y-F \& Hori Y (1995b). c-Fos Expression Mediated by N-Methyl-d-aspartate Receptors Following Anodal Polarization in the Rat Brain. Exp Neurol 133, 25-31.

Jennum P, Winkel H \& Fuglsang-Frederiksen A (1995). Repetitive magnetic stimulation and motor evoked potentials. Electroencephalogr Clin Neurophysiol Mot Control 97, 96-101.

Kidgell DJ, Daly RM, Young K, Lum J, Tooley G, Jaberzadeh S, Zoghi M \& Pearce AJ (2013). Different Current Intensities of Anodal Transcranial Direct Current Stimulation Do Not Differentially Modulate Motor Cortex Plasticity. Neural Plast 2013, 1-9.

Kirkwood A \& Bear MF (1994). Homosynaptic Long-Term Depression in the Visual Cortex. J Neurosci.

Kleim JA, Barbay S \& Nudo RJ (1998). Functional Reorganization of the Rat Motor Cortex Following Motor Skill Learning. J Neurophysiol.

Kuo M-F \& Nitsche M a. (2012). Effects of Transcranial Electrical Stimulation on Cognition. Clin EEG Neurosci 43, 192-199.

Kuo M-F, Paulus W \& Nitsche MA (2006). Sex differences in cortical neuroplasticity in humans. Neuroreport 17, 1703-1707. 
Kuo MF, Paulus W \& Nitsche MA (2014). Therapeutic effects of non-invasive brain stimulation with direct currents (tDCS) in neuropsychiatric diseases. Neuroimage 85, 948-960.

Lang N, Siebner HR, Ward NS, Lee L, Nitsche MA, Paulus W, Rothwell JC, Lemon RN \& Frackowiak RS (2005). How does transcranial DC stimulation of the primary motor cortex alter regional neuronal activity in the human brain? Eur J Neurosci 22, 495-504.

Li H-J, Hou X-H, Liu H-H, Yue C-L, Lu G-M \& Zuo X-N (2015). Putting agerelated task activation into large-scale brain networks: A meta-analysis of 114 fMRI studies on healthy aging. Neurosci Biobehav Rev 57, 156-174.

Liebetanz D, Nitsche MA, Tergau F \& Paulus W (2002). Pharmacological approach to the mechanisms of transcranial DC-stimulation-induced after-effects of human motor cortex excitability. Brain 125, 2238-2247.

Lisman JE (2001). Three Ca2+ levels affect plasticity differently: The LTP zone, the LTD zone and no man's land. J Physiol 532, 285.

López-Alonso V, Cheeran B, Río-Rodríguez D \& Fernández-Del-Olmo M (2014). Inter-individual variability in response to non-invasive brain stimulation paradigms. Brain Stimul 7, 372-380.

Malenka RC \& Bear MF (2004). LTP and LTD: an embarrassment of riches. Neuron 44, 5-21.

Merton PA \& Morton HB (1980). Stimulation of the cerebral cortex in the intact human subject. Nature 285, 227.

Misonou H, Mohapatra DP, Park EW, Leung V, Zhen D, Misonou K, Anderson AE \& Trimmer JS (2004). Regulation of ion channel localization and phosphorylation by neuronal activity. Nat Neurosci 7, 711-718.

Miyamoto E (2006). Molecular mechanism of neuronal plasticity: induction and maintenance of long-term potentiation in the hippocampus. $J$ Pharmacol Sci 100, 433-442.

Moliadze V, Schmanke T, Andreas S, Lyzhko E, Freitag CM \& Siniatchkin M (2015). Stimulation intensities of transcranial direct current stimulation have to be adjusted in children and adolescents. Clin Neurophysiol 126, 1392-1399.

Monte-Silva K, Kuo MF, Hessenthaler S, Fresnoza S, Liebetanz D, Paulus W \& Nitsche MA (2013). Induction of late LTP-like plasticity in the human motor cortex by repeated non-invasive brain stimulation. Brain Stimul 6, 424-432.

Nitsche MA, Cohen LG, Wassermann EM, Priori A, Lang N, Antal A, Paulus W, Hummel F, Boggio PS, Fregni F \& Pascual-Leone A (2008). Transcranial direct current stimulation: State of the art 2008. Brain Stimul 1, 206-223.

Nitsche MA, Fricke K, Henschke U, Schlitterlau A, Liebetanz D, Lang N, Henning S, Tergau F \& Paulus W (2003). Pharmacological modulation of cortical excitability shifts induced by transcranial direct current stimulation in humans. J Physiol 553, 293-301.

Nitsche MA, Liebetanz D, Schlitterlau A, Henschke U, Fricke K, Frommann K, Lang N, Henning S, Paulus W \& Tergau F (2004). GABAergic 
modulation of DC stimulation-induced motor cortex excitability shifts in humans. Eur J Neurosci 19, 2720-2726.

Nitsche MA \& Paulus W (2000). Excitability changes induced in the human motor cortex by weak transcranial direct current stimulation. J Physiol 527 Pt 3, 633-639.

Nitsche MA \& Paulus W (2001). Sustained excitability elevations induced by transcranial DC motor cortex stimulation in humans Sustained excitability elevations induced by transcranial DC motor cortex stimulation in. Neurology 57, 1899-1901.

Notturno F, Marzetti L, Pizzella V, Uncini A \& Zappasodi F (2014). Local and remote effects of transcranial direct current stimulation on the electrical activity of the motor cortical network. Hum Brain Mapp 35, 2220-2232.

Opitz A, Paulus W, Will A \& Thielscher A (2015). Anatomical determinants of the electric field during transcranial direct current stimulation. Neuroimage 109, 2.

Pascual-Leone A, Amedi A, Fregni F \& Merabet LB (2005). The Plastic Human Brain Cortex. Annu Rev Neurosci 28, 377-401.

Pascual-Leone A, Freitas C, Oberman L, Horvath JC, Halko M, Eldaief M, Bashir S, Vernet M, Shafi M, Westover B, Vahabzadeh-Hagh AM \& Rotenberg A (2011). Characterizing brain cortical plasticity and network dynamics across the age-span in health and disease with TMS-EEG and TMS-fMRI. Brain Topogr 24, 302-315.

Pascual-Leone A, Nguyet D, Cohen LG, Brasil-Neto JP, Cammarota A \& Hallett M (1995). Modulation of muscle responses evoked by transcranial magnetic stimulation during the acquisition of new fine motor skills. $J$ Neurophysiol 74, 1037-1045.

Pascual-Leone A, Valls-sole J, Wassermann EM \& Hallett M (1994). Responses to rapid-rate transcranial magnetic stimulation of the human motor cortex. Brain 117, 847-858.

Pereda AE, Bell TD, Chang BH, Czernik AJ, Nairn AC, Soderling TR \& Faber DS (1998). Ca2+/calmodulin-dependent kinase II mediates simultaneous enhancement of gap-junctional conductance and glutamatergic transmission. Proc Natl Acad Sci U S A 95, 13272-13277.

Ploughman M (2002). A review of brain neuroplasticity and implications for the physiotherapeutic management of stroke. Physiother Canada 54, $164-176,185$.

Polanía R, Nitsche MA \& Paulus W (2011a). Modulating functional connectivity patterns and topological functional organization of the human brain with transcranial direct current stimulation. Hum Brain Mapp 32, 1236-1249.

Polanía R, Nitsche MA \& Paulus W (2011b). Modulating functional connectivity patterns and topological functional organization of the human brain with transcranial direct current stimulation. Hum Brain Mapp 32, 1236-1249.

Polanía R, Paulus W, Antal A \& Nitsche MA (2011c). NeuroImage Introducing graph theory to track for neuroplastic alterations in the resting human brain: A transcranial direct current stimulation study. Neuroimage 54, 2287-2296. 
Purpura DP \& McMurtry JG (1965). Intracellular Activities and Evoked Potential Changes During of motor cortex. Neurophysiol 28, 166-185.

Ridding MC \& Ziemann U (2010). Determinants of the induction of cortical plasticity by non-invasive brain stimulation in healthy subjects. J Physiol 588, 2291-2304.

Rioult-Pedotti M-S, Friedman D, Hess G \& Donoghue JP (1998). Strengthening of horizontal cortical connections following skill learning. Nat Neurosci 1, 230-234.

Rioult-Pedotti MS, Friedman D \& Donoghue JP (2000). Learning-induced LTP in neocortex. Science 290, 533-536.

Rothwell JC (1993). Evoked potentials, magnetic stimulation studies, and event-related potentials. Curr Opin Neurol 6, 715-723.

Roy A, Baxter B \& He B (2014). High-Definition Transcranial Direct Current Stimulation Induces Both Acute and Persistent Changes in Broadband Cortical Synchronization : A Simultaneous tDCS - EEG Study. 61, 19671978.

Sanes JN \& Donoghue JP (2000). Plasticity and primary motor cortex. Annu Rev Neurosci 23, 393-415.

Sheiner LB, Rosenberg B \& Melmon KL (1972). Modelling of individual pharmacokinetics for computer-aided drug dosage. Comput Biomed Res 5, 441-459.

Shekhawat GS, Stinear CM \& Searchfield GD (2013). Transcranial direct current stimulation intensity and duration effects on tinnitus suppression. Neurorehabil Neural Repair 27, 164-172.

Shin Y-I, Foerster Á \& Nitsche MA (2015). Transcranial direct current stimulation (tDCS) - application in neuropsychology. Neuropsychologia 69, 154-175.

Sporns O \& Honey CJ (2006). Small worlds inside big brains. Proc Natl Acad Sci U S A 103, 19219-19220.

Stagg CJ, Best JG, Stephenson MC, O’Shea J, Wylezinska M, Kincses ZT, Morris PG, Matthews PM \& Johansen-Berg H (2009). Polarity-Sensitive Modulation of Cortical Neurotransmitters by Transcranial Stimulation. $J$ Neurosci 29, 5202-5206.

Stagg CJ, Bestmann S, Constantinescu AO, Moreno LM, Allman C, Mekle R, Woolrich M, Near J, Johansen-Berg H, Rothwell JC, Moreno Moreno L, Allman C, Mekle R, Woolrich M, Near J, Johansen-Berg H \& Rothwell JC (2011). Relationship between physiological measures of excitability and levels of glutamate and GABA in the human motor cortex. J Physiol 589, 5845-5855.

Stagg CJ, Lin RL, Mezue M, Segerdahl A, Kong Y, Xie J \& Tracey I (2013). Widespread modulation of cerebral perfusion induced during and after transcranial direct current stimulation applied to the left dorsolateral prefrontal cortex. J Neurosci 33, 11425-11431.

Stagg CJ \& Nitsche MA (2011). Physiological Basis of Transcranial Direct Current Stimulation. Neurosci 17, 37-53.

Stefan K, Kunesch E, Benecke R, Cohen LG \& Classen J (2002). Mechanisms of enhancement of human motor cortex excitability induced by interventional paired associative stimulation. J Physiol 543, 699-708. 
Stefan K, Kunesch E, Cohen LG, Benecke R \& Classen J (2000). Induction of plasticity in the human motor cortex by paired associative stimulation. Brain 123 Pt 3, 572-584.

Stelzer a, Simon G, Kovacs G \& Rai R (1994). Synaptic disinhibition during maintenance of long-term potentiation in the CA1 hippocampal subfield. Proc Natl Acad Sci U S A 91, 3058-3062.

Stelzer A, Slater NT \& ten Bruggencate G (1987). Activation of NMDA receptors blocks GABAergic inhibition in an in vitro model of epilepsy. Nature 326, 698-701.

Strube W, Bunse T, Malchow B \& Hasan A (2015). Efficacy and Interindividual Variability in Motor-Cortex Plasticity following Anodal tDCS and Paired-Associative Stimulation. Neural Plast 2015, 1-10.

Swinnen SP (2002). Intermanual Coordination: From Behavioural Principles To Neural-Network Interactions. Nat Rev Neurosci 3, 348-359.

Swinnen SP, Jardin K, Meulenbroek R, Dounskaia N \& Den Brandt MH-V (1997). Egocentric and Allocentric Constraints in the Expression of Patterns of Interlimb Coordination. J Cogn Neurosci 9, 348-377.

Ugur HC, Kahilogullari G, Coscarella E, Unlu A, Tekdemir I, Morcos JJ, Elhan A \& Baskaya MK (2005). Arterial vascularization of primary motor cortex (precentral gyrus). Surg Neurol 64, 48-52.

Wiethoff S, Hamada M \& Rothwell JC (2014). Variability in response to transcranial direct current stimulation of the motor cortex. Brain Stimul 7, 468-475.

Winnubst J, Cheyne JE, Niculescu D \& Lohmann C (2015). Spontaneous Activity Drives Local Synaptic Plasticity InVivo. Neuron 87, 399-411.

Wolters A, Schmidt A, Schramm A, Zeller D, Naumann M, Kunesch E, Benecke R, Reiners K \& Classen J (2005). Timing-dependent plasticity in human primary somatosensory cortex. J Physiol 565, 1039-1052.

Woods AJ et al. (2016). A technical guide to tDCS, and related non-invasive brain stimulation tools. Clin Neurophysiol 127, 1031-1048.

Zheng X, Alsop DC \& Schlaug G (2011). Effects of transcranial direct current stimulation (tDCS) on human regional cerebral blood flow. Neuroimage 58, 26-33.

Zimerman M \& Hummel FC (2010). Non-Invasive Brain Stimulation: Enhancing Motor and Cognitive Functions In Healthy Old Subjects. Front Aging Neurosci

2, $1-12$. 


\section{Asif Jamil}

Nationality: US-American

Education

University of Göttingen, Göttingen, Germany

Ph.D, Systems Neuroscience (Expected 2016)

King's College London, Institute of Psychiatry, London, United Kingdom

MSc, Neuroimaging (Sept, 2012)

University of Maryland, College Park Maryland, United States

BSc, Biology (May, 2011)

\section{Peer-Reviewed Publications}

Jamil A, Batsikadze G, Kuo H-I, Labruna L, Hasan A, Paulus W \& Nitsche MA (2016). Systematic evaluation of the impact of stimulation intensity on neuroplastic after-effects induced by transcranial direct current stimulation. J Physiol 0, 1-16.

Kuo H-I, Paulus W, Batsikadze G, Jamil A, Kuo M-F \& Nitsche MA (2016). Acute and chronic effects of noradrenergic enhancement on transcranial direct current stimulation (tDCS)-induced neuroplasticity in humans. J Physiol; DOI: 10.1113/JP273137.

Labruna L, Jamil A, Fresnoza S, Batsikadze G, Kuo M-F, Vanderschelden B, Ivry RB \& Nitsche MA (2015). Efficacy of Anodal Transcranial Direct Current Stimulation is Related to Sensitivity to Transcranial Magnetic Stimulation. Brain Stimul 9, 8-15.

Kuo H-I, Paulus W, Batsikadze G, Jamil A, Kuo M-F \& Nitsche M a (2015). Chronic enhancement of serotonin facilitates excitatory transcranial direct current stimulation-induced neuroplasticity. Neuropsychopharmacology 1-39.

\section{Research Projects}

October 2015 - Present

Research on "Identification and modulation of age-related alterations of functional networks in bimanual visual-motor coordination.", KU Leuven \& University of Hasselt, Belgium.

- Using EEG to understand the neurophysiological correlates of bimanual coordination, and how these are affected by aging.

- Devise a model of transcranial direct current stimulation (tDCS) to optimally facilitate underperforming or deficient cortical networks, which may improve bimanual coordination.

- Understand the causal role of enhanced motor cortical coordination network by tDCS using combined tDCS and EEG.

Skills: EEG application and analysis, tDCS application, bimanual motor learning, kinematic and EMG analysis, MATLAB

March 2013 - Present

Research on "Optimizing the efficacy of transcranial direct current stimulation on cortical neuroplasticity based on a neurovascular coupling model" University of Göttingen, Germany. - Obtain an optimal protocol of non-invasive brain stimulation to alter cortical neuroplasticity by deriving a physiology-based model of the human cortex. This model should examine the 
relationship between the effects of tDCS on cortical excitability (measured as "evoked potentials" using transcranial magnetic stimulation (TMS)) and the effects on cerebral blood flow (measured using functional magnetic resonance imaging (fMRI)). The extent of the relationship

("neurovascular coupling") is determined from titrating the current intensity of the stimulation.

- Physiologically validate the "optimized" model on functional effects against "classical" stimulation protocol by measuring motor reaction time in a group of healthy subjects.

Skills: tDCS, MRI, TMS, EEG, SPM (MATLAB) analysis, SPSS statistical analysis.

\section{January 2012 - August 2012}

Research on "Enhancing Working Memory in Healthy Controls by applying transcranial Direct

Current Stimulation (tDCS)" King's College, London.

- Performed a neuroimaging study to investigate the effects of non-invasive brain stimulation in improving working memory. The study was part of a larger ongoing study to investigate tDCS as a therapy for patients deficient in this ability (ex: depression, Alzheimer's, schizophrenia).

- Analyzed the resulting data with SPM-8 (Statistical Parametric Mapping) for resting state BOLD analysis and SPSS for statistical analysis.

Skills: tDCS, MRI, SPM (MATLAB) analysis, SPSS statistical analysis.

December 2009 - May 2011

Research on "3D Auditory Reconstruction of Praying Mantises." University of Maryland

- Worked with neurophysiology professor to study the meso and meta ear of different species of praying mantises to understand vital auditory processes of ultrasound perception.

- Traced and triangulated different sets of two-dimensional x-ray synchrotron images of mantises acquired from the Argonne National Laboratory in Chicago to to create a 3D model of the organism

- Performed additional studies to calculate the distances in the tracheal air sacs so that a hearing frequency function could be computed.

Skills: Amira 3D Software, Blender 3D Software, 3D Reconstruction \& Segmentation

\section{Conference Presentations}

"Polarity \& intensity-dependent association between cortical excitability and CBF in tDCS-induced neuroplasticity", Poster at the 2015 Organization of Human Brain Mapping (OHBM) Conference presented June 2015 in Honolulu, Hawaii (USA).

"Optimizing transcranial Direct Current Stimulation (tDCS): Impact of baseline excitability", Poster presentation at the 2014 International Congress of Clinical Neurophysiology (ICCN) presented March 2014 in Berlin (Germany).

"Investigation of transcranial Direct Current Stimulation (tDCS) on enhancing Working Memory (WM) in healthy subjects: A pilot MRI study", Poster presentation, presented September, 2012 in King's College, London (UK).

"3D reconstruction of one-eared and two-eared Praying Mantises", Poster at the 2010 SICB Conference, Salt Lake City, Utah (USA).

"Hearing in the Praying Mantis", Poster at the 2011 University of Maryland Bioscience Day, College Park, Maryland (USA).

"Development of DNA Electrofractionation System", Poster at the 2009 University of Maryland College Park Scholars Presentation Day, College Park, Maryland (USA). 